Prepared for the U.S. Department of Energy under Contract DE-AC05-76RL01830

\title{
Characterization of Vadose Zone Sediment: Borehole 299-W23-19 [SX-115] in the S-SX Waste Management Area
}
RJ Serne
RE Clayton
IV Kutnyakov
BN Bjornstad
VL LeGore
DS Burke
DC Lanigan
MJ O'Hara
TC Wilson
GW Gee
CF Brown
BA Williams
CW Lindenmeier
GV Last

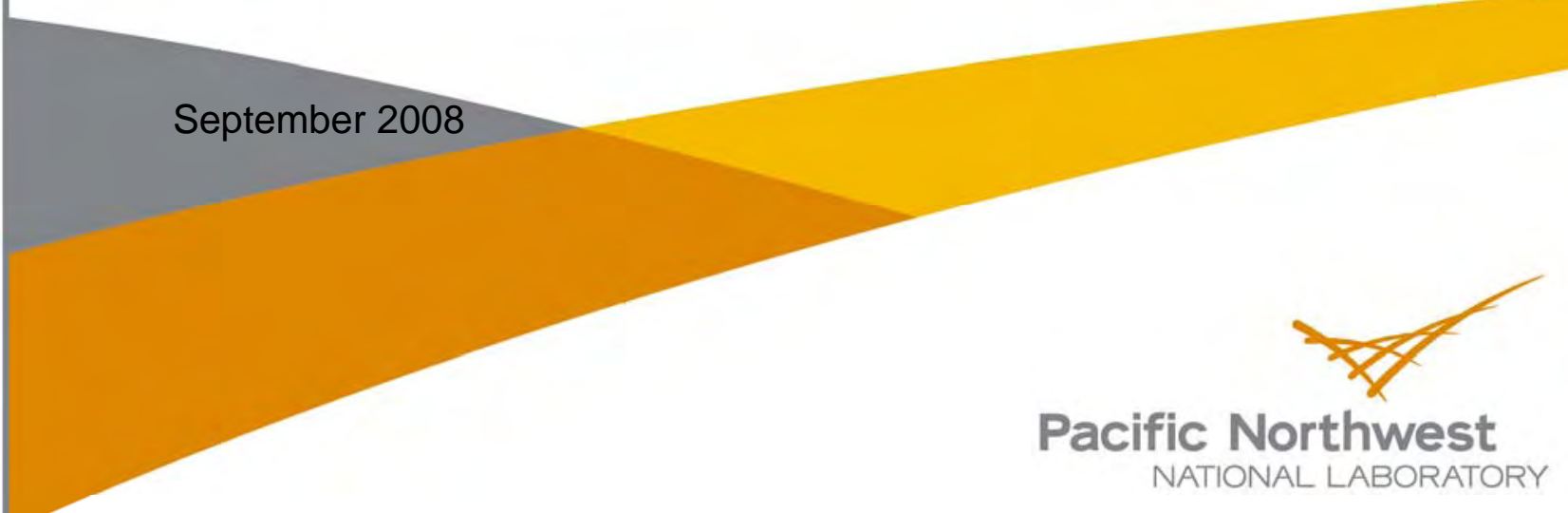




\title{
DISCLAIMER
}

This report was prepared as an account of work sponsored by an agency of the United States Government. Neither the United States Government nor any agency thereof, nor Battelle Memorial Institute, nor any of their employees, makes any warranty, express or implied, or assumes any legal liability or responsibility for the accuracy, completeness, or usefulness of any information, apparatus, product, or process disclosed, or represents that its use would not infringe privately owned rights. Reference herein to any specific commercial product, process, or service by trade name, trademark, manufacturer, or otherwise does not necessarily constitute or imply its endorsement, recommendation, or favoring by the United States Government or any agency thereof, or Battelle Memorial Institute. The views and opinions of authors expressed herein do not necessarily state or reflect those of the United States Government or any agency thereof.

\author{
PACIFIC NORTHWEST NATIONAL LABORATORY \\ operated by \\ BATTELLE \\ for the \\ UNITED STATES DEPARTMENT OF ENERGY \\ under Contract DE-AC06-76RL01830
}

This document was printed on recycled paper. 
PNNL-13757-2, Rev. 1

\section{Characterization of Vadose Zone Sediment: Borehole 299-W23-19 [SX-115] in the S-SX Waste Management Area}
R. J. Serne
R. E. Clayton
I. V. Kutnyakov
H. T. Schaef
V. L. LeGore
D. S. Burke
B. N. Bjornstad
R. D. Orr
T. C. Wilson
D. C. Lanigan
M. J. O'Hara
B. A. Williams
G. W. Gee
C. F. Brown
C. W. Lindenmeier
G. V. Last

September 2008

Prepared for CH2M HILL Hanford Group, Inc. and the U.S. Department of Energy under Contract DEAC06-76RL01830

Pacific Northwest National Laboratory

Richland, Washington 99352 


\section{Executive Summary}

This report was revised in September 2008 to remove acid-extractable sodium data from Tables 4.15 and 4.19. The sodium data was removed due to potential contamination introduced during the acid extraction process. The rest of the text remains unchanged from the original report issued in February 2002.

The Tank Farm Vadose Zone Project is led by CH2M HILL Hanford Group, Inc. Their goals include defining risks from past and future single-shell tank farm activities, identifying and evaluating the efficacy of interim measures, and collecting geotechnical information and data. The purpose of these activities is to support future decisions made by the U.S. Department of Energy (DOE) regarding near-term operations, future waste retrieval, and final closure activities for the single-shell tank Waste Management Areas. To help in this effort, CH2M HILL Hanford Group, Inc. contracted with scientists at Pacific Northwest National Laboratory to analyze sediment samples collected from borehole 299-W23-19.

Borehole 299-W23-19 was drilled at the southwestern edge of tank SX-115 as a characterization borehole to evaluate the depth distribution of contamination leaked from tank SX-115. The borehole was cored throughout the vadose zone during drilling. The geology, stratigraphy, and lithology of the drill core were described in the field and in the laboratory. The drill cores were sampled for analyses of physical, chemical, and hydraulic properties in order to better understand the distribution of contaminants and the mechanisms of contaminant movement beneath the SX tank farm.

Electrical conductivity and chromium, nitrate, sodium, and technetium concentrations were found to be good indicators of the plume distribution. Data for the other measured contaminants indicate that leaked tank fluids have impacted the vadose zone beneath tank SX-115 from about 22.2 meters (73 feet) depth to the base of the upper Plio-Pleistocene unit at about a 47.5 meters (156 feet) depth. The technetium-99 plume, however, appears to occur as deep as 62.5 meters (205 feet), which is the depth of the deepest sample.

The bulk of the leaked fluid beneath tank SX-115 resides in the upper Plio-Pleistocene unit between a 38.1 meters (125-feet) and 47.5 meters (156-foot) depth. This is slightly deeper than the contamination investigated at tank SX-108 and tank SX-109, both of which have the bulk of contamination in the shallower Hanford formation.

Results of chemical analyses point to three potential mechanisms influencing the distribution of contaminants in the vadose zone. Common ion exchange reactions appear to have influenced the distribution of most mobile contaminants whereas oxidation-reduction reactions seem to have influenced chromium distribution. Observations from this study suggest that nitrate, technetium-99, and perhaps molybdenum migrate with no measurable retardation in the vadose zone whereas sodium, chromium, and selenium migrate with a small amount of retardation. These conclusions are similar to the conclusions found during the SX-108 and SX-109 tank studies.

The conclusions reached from this study support specific mechanisms influencing subsurface migration of contaminants. The mechanisms are supported by the distributions of contaminants beneath tank farms. These observations will help DOE and CH2M HILL Hanford Group, Inc. identify and implement viable remediation and closure activities. 



\section{Acknowledgements}

The authors from Pacific Northwest National Laboratory wish to thank the Tank Farm Vadose Zone Project Team at CH2M HILL Hanford Group, Inc., for the monetary support and technical exchange during the planning and execution of the work described in this document. The team is led by Tony Knepp with support from Dave Myers, Tom Jones, Marc Wood, Fred Mann, Raz Khaleel, and Harold Sydnor. Marc Wood, Dave Myers, Fred Mann, and Raz Khaleel provided technical and editorial review for these borehole characterization reports.

We wish to thank the field drilling crew led by Marty Gardner and Kent Reynolds for their ingenuity and perseverance at obtaining the core samples under difficult health and safety and radiation protection conditions.

We wish to thank Dr. Kevin Lindsey, currently employed by Kennedy/Jenks Consultants, Richland, Washington, for sharing his geologic descriptions of the core samples and for his technical comments on the draft report.

We thank Duane Horton for providing internal technical review for this document. 


\section{Contents}

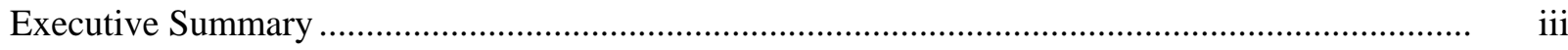

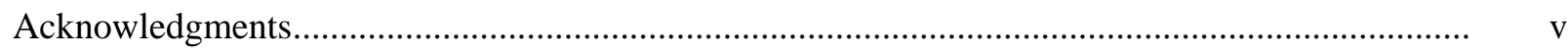

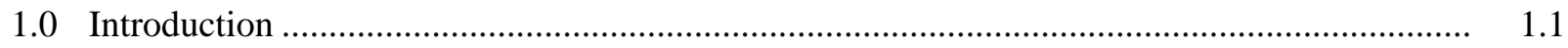

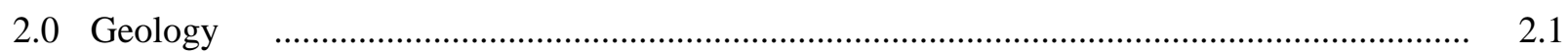

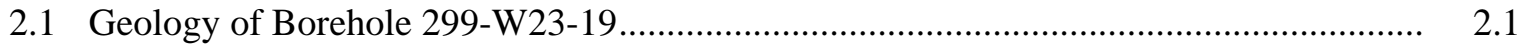

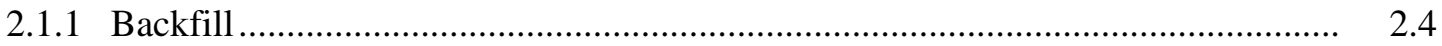

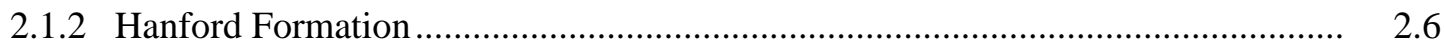

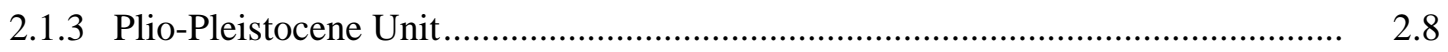

2.1.4 Ringold Formation .............................................................................. 2.10

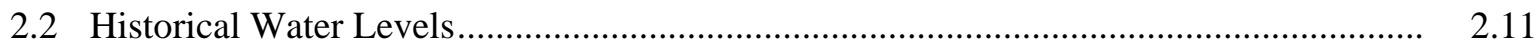

3.0 Geochemical Method and Materials ......................................................................... 3.1

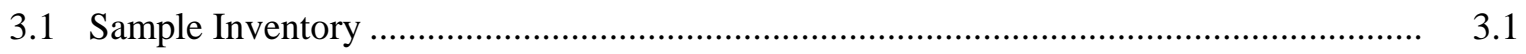

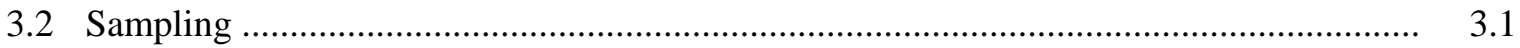

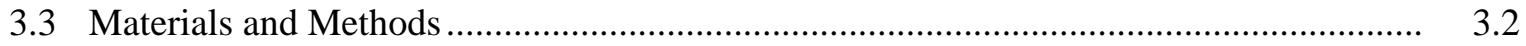

3.3.1 Moisture Content........................................................................................ 3.2

3.3.2 1:1 Sediment-to-Water Extract...................................................................... 3.3

3.3.3 Porewater Composition ............................................................................ 3.4

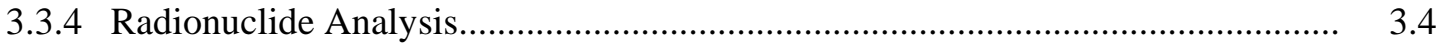

3.3.5 Carbon Content ........................................................................................ 3.5

3.3.6 $8 \mathrm{M}$ Nitric Acid Extract .................................................................................. 3.6

3.3.7 Elemental Analysis............................................................................. 3.6

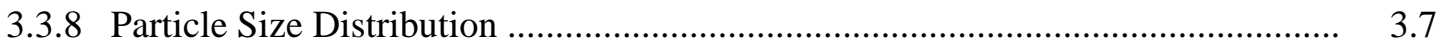

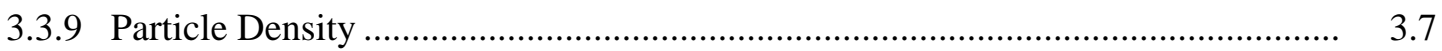

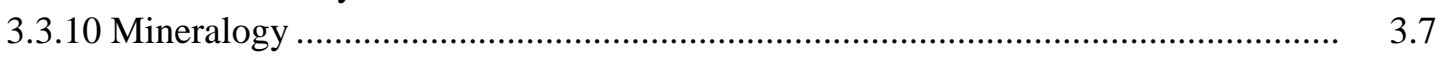

3.3.11 Water Potential (Suction) Measurements ...................................................... 3.8

3.3.12 Semi-Volatile Organic Analyses ...................................................................... $\quad 3.9$

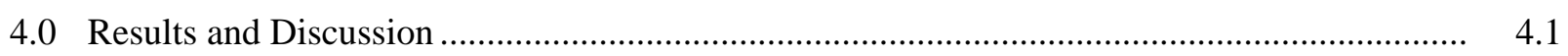

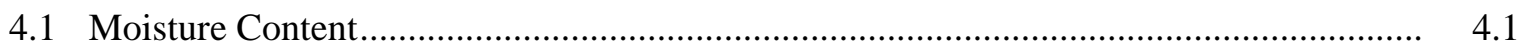

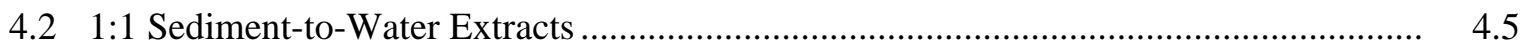

4.2.1 pH and Electrical Conductivity ............................................................. 4.5

4.2.2 Anions and Cations ..................................................................................... 4.7

4.2.3 Radionuclide Content in Vadose Zone Sediment............................................... 4.31

4.2.4 Total Carbon, Balcium Carbonate, and Organic Carbon in Vadose Zone
Sediment from Borehole 299-W23-19 ............................................................ 
4.2.5 8 M Nitric Acid-Extractable Amounts of Selected Elements.

4.2.6 Sediment Total Oxide Composition .................................................................. 4.43

4.2.7 Particle Size Measurements on Vadose Zone Sediment ..................................... 4.46

4.2.8 Particle Density of Bulk Sediment Samples.......................................................... 4.52

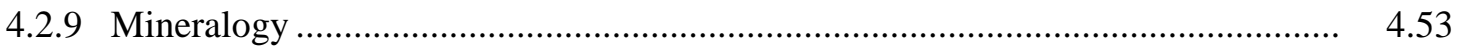

4.2.10 Water Potential or Soil Suction ............................................................................. 4.58

4.2.11 Results of Simi-Volatile Organic Analyses

5.0 Contaminant Distribution at Borehole 299-W23-19 as Compared with Contaminant Distribution at Nearby Wells

5.1 In Situ Distribution Coefficients

5.2 Contaminant Distribution in Borehole 200-W23-19 and other Nearby Wells

6.0 Summary and Conclusion

6.1 Physical Model of the Hydrogeology at Borehole 299-W23-19.

6.2 Vertical Extent of Contamination.

6.3 Estimate of Sediment Recharge Rates (Matric Potential). 6.6

6.4 Detailed Characterization to Clarify Controlling Geochemcial Processes.. 6.6

6.5 Estimates of Sorption-Desorption Values

6.6 Other Characterization Observations 


\section{Figures}

2.1 Location Map for well 299-W23-19 (B8809) at cross section A-A1 at the 241-SX tank farm

2.2 Lithology, Stratigraphy, and Moisture Distribution as a Function of Depth Within Borehole 299-W23-19

2.3 Cross Section through 241-SX Tank Farm and Borehole 299-W23-19 ............................ 2.5

2.4 Disaggregated core from the Hanford formation H1 unit (85.5-86.5 ft depth) in Borehole 299-W23-19

2.5 Disaggregated Core from the Hanford H2 formation unit (112.0-112.5 ft depth) in Borehole 299-W23-19

2.6 Partially Disaggregated Core from the Upper Plio-Pleistocene Unit (145.1-145.6 ft depth) in Borehole 299-W23-19

2.7 Disaggregated Core from the Lower Plio-Pleistocene Unit (156.1-156.6 ft depth) in Borehole 299-W23-19

2.8 Disaggregated Core from Ringold Formation Unit E (184.2-184.9 ft depth) in Borehole 299-W23-19

2.9 Historic Water Levels Beneath the 241-SX Tank Farm

4.1 Moisture Content in Vadose Zone Sediment Profile at Borehole 299-W23-19

4.2 $\mathrm{pH}$ and Electrical Conductivity for Dilution-Corrected and Actual Porewaters from Borehole 299-W23-19

4.3 Dilution-Corrected (from sediment:water extracts) and Actual (from ultracentrifuge)

Porewater Anion Concentrations for Borehole 299-W23-19 Sediment

4.4 Cations Calculated (from sediment:water extracts) and Actual Porewaters for Borehole 299-W23-19 Sediment

4.5 Trace Metals Concentrations versus Depth in Dilution-Corrected (from sediment:water extracts) and Actual (from ultracentrifuge) Porewaters from Borehole 299-W23-19 Sediment

4.6 Water- and Acid-Extractable Technetium-99 in Sediment from Borehole 299-W23-19 ..... 4.37

4.7 Uranium Extracted from Sediment (water versus acid) .............................................. 4.38 
4.8 Particle-Size Distribution of Samples from the Hanford Formation H1a Unit in

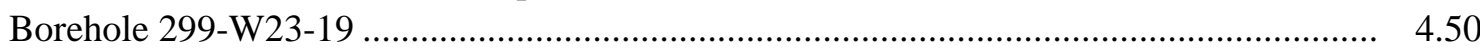

4.9 Particle-Size Distribution of Samples from the Hanford Formation H1 and H2 Units in

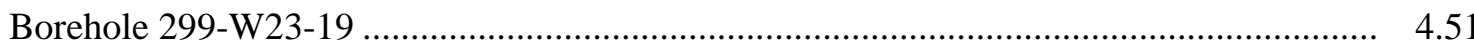

4.10 Article-Size Distribution of Samples from the Upper Plio-Pleistocene Unit in Borehole 299-W23-19

4.11 Particle-Size Distribution of a Sample of Ringold Formation, Member of Wooded Island Unit E from Borehole 299-W23-19.

4.12 Typical Illite Particle from Sample 31G

4.13 Image of Clay Particles in Sample 31G. The dense smectite aggregate (middle, left) is easily distinguished from the other clay minerals

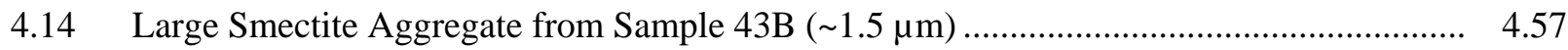

4.15 Kaolinite Particle (Bottom) with Trace Amounts of Iron (III) $(\sim 1 \mu \mathrm{m})$ and Platey Illite Particle (Top) from Sample 22D.

4.16 Soil Suction Profiles for Boreholes 299-W22-48, 299-W22-50, 299-W23-19. Dashed line is the equilibrium soil suction line. Drainage occurs only for values left of the equilibrium line

5.1 Stratigigraphic sequences encountered in five new boreholes at the S-SX tank farms

5.2 $\mathrm{pH}$ versus depth in five new boreholes at the SX tank farm

5.3 Dilution corrected electrical conductivity versus depth in five new boreholes at the SX tank farm

5.4 Dilution corrected nitrate concentrations versus depth in five new boreholes at the SX tank farm

5.5 Dilution corrected sodium concentrations versus depth in five new boreholes at the SX tank farm

5.6a Alkali and alkaline-earth metal concentrations versus depth in four new boreholes at the SX tank farm. 
5.6c no caption.

5.6d no caption.

5.7 Dilution corrected technetium -99 concentrations versus depth in three new boreholes at the SX tank farm

\section{Tables}

4.1 Moisture Content of Sediment from Borehole 299-W232-19 ........................................... 4.2

4.2 Water Extract pH and Electrical Conductivity Values ................................................... 4.6

4.3 Comparison of Actual Porewater $\mathrm{pH}$ and Electrical Conductivity with Dilution-corrected

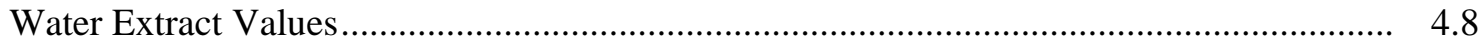

4.4 Anion Concentrations of Water Extracts from Borehole 299-W23-19 Sediment Samples.... 4.12

4.5 Comparison of Dilution-corrected and Actual Porewater Anion Concentrations in Vadose Zone Sediment from Borehole 299-W23-19

4.6 Calculated Porewater Cation Composition from Water Extracts from Vadose Zone Sediment from 299-W23-19 Borehole

4.7 Cation Concentrations of Actual and Dilutaion-corrected Porewaters

4.8 Trace Metal Concentrations of Actual and Dilution-corrected Porewaters in Sediment from Borehole 299-W23-19

4.9 Trace Metal Concentrations from Actual and Dilution-corrected Porewaters

4.10 Ratio of Some Major and Trace Metals to the Mobile Species Nitrate and Technetium-99.. 4.29

4.11 Gamma Energy Analysis of Vadose Zone Sediment........................................................ 4.32

4.12 Strontium-90 and Tritium Contents of Vadose Zone Sediment ...................................... 4.35

4.13 Technetium-99 and Uranium Content in Sediment .................................................... 4.35

4.14 Carbon Content in Vadose Sediment from 299-W23-19 …............................................. 4.40 
4.15 Acid-Extractable Major Element Concentrations of the Vadose Sediment from Borehole 299-W23-19.

4.16 Acid-Extractable Trace Element Concentrations of the Vadose Sediment from Borehole 299-W23-19.

4.17 Oxide Concentrations of Bulk Vadose Zone Sediment from Borehole 299-W23-19

4.18 Total Composition of the Clay-Sized Fraction of Sediment from Borehole 299-W23-19 wt $\%$ as Oxides

4.19 Percentage of Elements Extracted by 8 M Nitric Acid. 4.46

4.20 Dry Sieve Particle Size Distribution of Samples from Borehole 200-W23-19 ................... 4.47

4.21 Folk/Wentworth Classification of Sample from Borehole 299-W23-19............................ 4.48

4.22 Comparison of Particle Size Distribution Measured by the Wet Sieve/Hydrometer Method and the Dry Sieve Method

4.23 Particle Density of Bulk Sediment from 299-W23-19 Borehole

4.52

4.24 Semiquantitative Mineral Composition for Bulk Samples

4.25 Semiquantitative Analysis of the Clay Fraction of Samples from Borehole 299-W23-19.... 4.54

5.1 Quantification of Desorption Potential for Major Contaminants in Borehole 299-W23-19 .. 5.2 


\subsection{Introduction}

The Tank Farm Vadose Zone Project is led by CH2M HILL Hanford Group, Inc. with the goals of defining risks from past and future single-shell tank farm activities, identifying and evaluating the efficacy of interim measures, and collecting geotechnical information and data. The purpose of these activities is to support future decisions made by the U.S. Department of Energy regarding near-term operations, future waste retrieval, and final closure activities for the single-shell tank Waste Management Areas. A complete discussion of the goals of the Tank Farm Vadose Zone Project are in the overall work plan, Phase 1 RCRA Facility Investigation/Corrective Measures Study Work Plan for the Single-Shell Tank Waste Management Areas (DOE 1999).

This document is one in a series of four reports that describe the geology, geochemistry, and hydrology of sediment collected from five new boreholes in Waste Management Area S-SX. This report concentrates on results collected from borehole 299-W23-19. The other reports describe data collected from borehole 41-09-39 (299-W23-234), southeast of single-shell tank SX-109; borehole C3082, a slant borehole drilled through contaminated sediment under single-shell tank SX-108; and wells 299-W23-48 and 200-W23-50, drilled through uncontaminated sediment outside and east of the SX Tank Farm. The results of all of these investigations have been incorporated in an interpretation of the subsurface distribution of contamination at the S-SX Tank Farm as described in the S-SX Tank Forum Field Investigation Report (FIR).

The objective of this document is to present data collected from drill cores taken from well 299-W2319 located adjacent to the southwest edge of single-shell tank SX-115. Geologic, geochemical, and hydraulic data are presented and an interpretation of the data is made to (1) support an appropriate geologic conceptual model, (2) describe the vertical extent of contamination, (3) predict the migrationpotential of the contaminants that still reside in the vadose zone, and (4) compare and correlate the contaminant distribution in the borehole sediment with groundwater plumes in the aquifer proximate and down hydraulic gradient from the SX Tank Farm.

Borehole 299-W23-19 was originally drilled as a characterization borehole to evaluate the depth distribution of contamination. The borehole was turned into a Resource Conservation and Recovery Act - compliant groundwater monitoring well after a groundwater sample showed that the groundwater contained very high levels of technetium-99. The groundwater is now sampled quarterly as part of the Waste Management Area S-SX monitoring network.

This report is divided into several sections: this introduction, a description of the geologic characteristics at Waste Management Area S-SX, the laboratory characterization methods used to obtain geochemical parameters, the results and a discussion of the results of the investigation, a comparison of contaminant distribution, a summary and conclusions, and the references cited.

English units are used in this report for depths because that is the system of units used by drillers to

measure and report depths and well construction details. To convert feet to meters, multiply by 0.3048 ; to convert inches to centimeters multiply by 2.54 . Metric units are used for all other measurements. 


\subsection{Geology}

The geology of the Hanford Site and the S and SX tank farms is discussed at length in Serne et al. (2002a). This report describes only the geology encountered by borehole 299-W23-19.

\subsection{Geology of Borehole 299-W23-19}

Borehole 299-W23-19 is located in the southwest corner of the SX Tank Farm, 3 meters (10 feet) southwest of single-shell tank SX-115 (Figure 2.1) where the vadose zone is 64.6 meters (212 feet) thick (Figure 2.2). Tank SX-115 is a confirmed leaker. Lindsey et al. (2000) presented a detailed account of the drilling, sampling, geophysical logging, and geology of borehole 299-W23-19. Much of the following discussion is taken from and/or built upon the information in Lindsey et al (2000).

Borehole 299-W23-19 (also referred to as borehole B8809) was installed between August 2 and September 2, 1999. This borehole was drilled using the reverse air-rotary method in a drive and drill mode. Near-continuous samples were collected through the Hanford formation by driving a split spoon sampler ahead of the casing string. Samples were acquired using a 1.5 meters (5 feet) split-spoon sampler containing 15- and/or 30-centimeters (6- and/or 12-inches) long steel liners. The depth to the bottom of the borehole was measured just prior to each sampling event.

After collection of each split-spoon, the borehole was reamed out using reverse air rotary methods and the casing advanced to the next sample location. All air-lifted cuttings were treated as if contaminated and all effluent air was passed through high-efficiency particulate air (HEPA) filters before being discharged to the atmosphere. No radioactivity above background was detected by field instruments during drilling.

The borehole was drilled to a total depth of 75 meters ( 246 feet). The borehole terminated in the Ringold Unit E gravels approximately 10.4 meters (34 feet) below the water table (Figure 2.2).

Four types of downhole geophysical logs were obtained from borehole 299-W23-19; these include: (1) high purity germanium (HPGe) spectral gamma, (2) neutron-neutron, (3) neutron-gamma, and (4) temperature logs (Lindsey et al. 2000). The spectral gamma log indicated the presence of cesium-137 contamination in the upper 1.5 meters ( 5 feet) of the borehole; no other manmade contaminants were detected (Lindsey et al. 2000). The total (i.e., gross) gamma and neutron-neutron logs are shown in Figure 2.2. An anomalous spike on the total-gamma log at a depth of 30 meters (100 feet) depth is likely the result of a short section of the borehole that was uncased during logging.

Split-spoon samples were collected wherever possible. Once the sampler was brought to the surface, the liners were removed, capped, taped, and placed in coolers for transport to the Pacific Northwest National Laboratory (PNNL) analytical laboratory at the 3720 Building. Once at the laboratory, the sampled materials were removed from the liners inside a fume hood by hammering on the sleeve until the sediment within the liner emptied into a plastic tray. Consequently, most of the samples were 


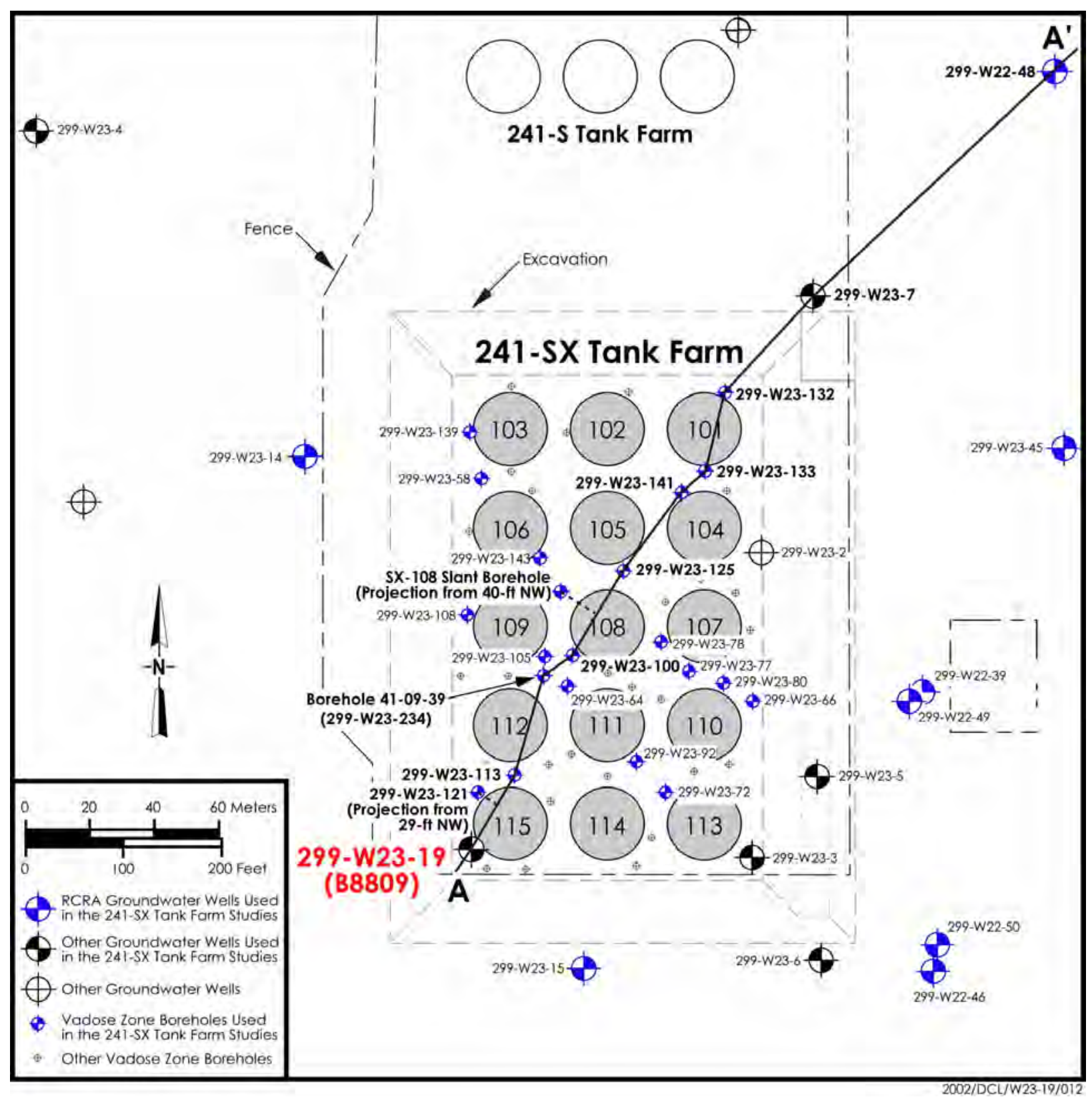

Figure 2.1 Location Map for well 299-W23-19 (B8809) and cross section A-A1 at the 241-SX Tank Farm.

disaggregated during the removal process. After removal from the core liner, the sample was described and photographed, and immediately subsampled for moisture content and various geochemical tests. The geologic methodology for descriptions of the individual split-spoon samples is presented in Lindsey et al. (2000). Once the descriptions were complete, the samples were sealed in individual plastic containers and returned to cold storage until additional studies were performed. 
299-W23-19 (B8809)

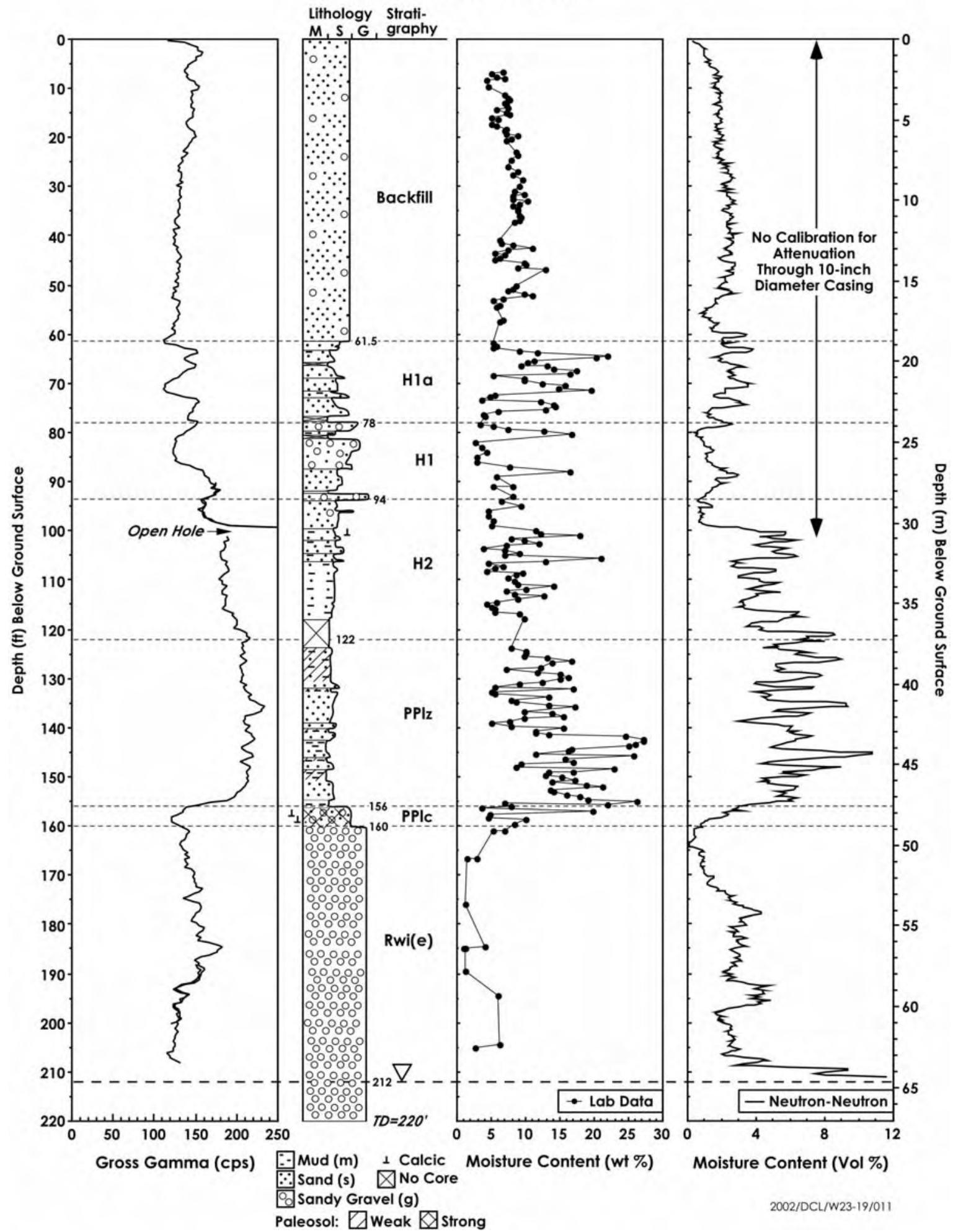

Figure 2.2 Lithology, Stratigraphy, and Moisture Distribution as a Function of Depth Within Borehole 299-W23-19. 
The 299-W23-19 borehole intersected four principal stratigraphic layers: (1) backfill around the highlevel waste tanks, (2) the Hanford formation, (3) the Plio-Pleistocene unit, and (4) the Ringold Formation. The lithology and stratigraphy is summarized in Figure 2.2. Figure 2.2 is based on data presented in Lindsey et al. (2000) as well as a more in-depth interpretation of the geophysical logs. In general, the same contact depths as those presented in Lindsey et al. (2000) are used. However, slight differences in the stratigraphic nomenclature for the Hanford formation and Plio-Pleistocene unit exist between Figure 2.2 and that presented in Lindsey et al. (2000), including: (1) subdivision of the Hanford formation, and (2) differentiation between the upper Plio-Pleistocene unit (PPlz) from the Hanford formation. As discussed in Serne et al. (2002a), we interpret along with Sobcyzk (2000) and Johnson et al. (1999), that the gravel unit (Hanford formation H1 unit) is continuous and correlatable beneath the SX Tank Farm (Figure 2.3). It is an important hydrostratigraphic unit that should be distinguished as a separate unit within the Hanford formation.

Another refinement of the interpretation of Lindsey et al. (2000) is the location of the top of the PlioPleistocene unit to be located at, or near, a shift in the background gamma counts at the 37.2 meters (122 feet) depth. The depth is chosen as the base of the Hanford formation. No core was recovered in the interval between 35.8 to 37.5 meters ( 117.5 to 123 feet) however, so the contact cannot be confirmed by samples. Nevertheless, there is a significant change in several geochemical parameters between 35.8 to 37.5 meters (117.5 to 123 feet) depth, (see Chapter 4, especially Figures 4.3 and 4.4). We attribute these changes in geochemistry to either: (1) lower permeability (i.e., higher moisture retention) materials, (2) difference in mineralogy, and/or (3) a difference in the degree of weathering and chemically reactive coatings on sediment grains. These shifts appear to be coincident with lithologic changes between the Hanford formation and the Plio-Pleistocene unit. A more detailed discussion of the geochemistry appears in Section 4.0. The following is a discussion of the geology of each of the stratigraphic units within borehole 299-W23-19.

\subsubsection{Backfill}

Backfill material from construction activities associated with the SX Tank Farm extends from the surface to a depth of 18.7 meters (61.5 feet). As described in Lindsey et al. (2000), this material consists of non-cohesive sand with variable silt and minor pebbles. One notable exception was a dry zone reported as being hard and difficult to sample at 1.5 meters ( 5 feet). A lack of variation in the geophysical logs (Figure 2.2) indicates the backfill material is relatively uniform and homogeneous throughout this stratigraphic unit.

A total of 70 samples were collected from intact cores of the backfill material for moisture content (see Section 4.0). The moisture content ranged from a few to up to $12 \mathrm{wt} \%$, but on average is between 5 to $10 \mathrm{wt} \%$. Four discrete intervals were analyzed for geochemical characterization from the backfill. Of interest is the notable lack of free (water-soluble) cations in the backfill compared to the underlying Hanford formation, from which the backfill was derived. The results of these analyses are discussed in more detail in Section 4.0. 


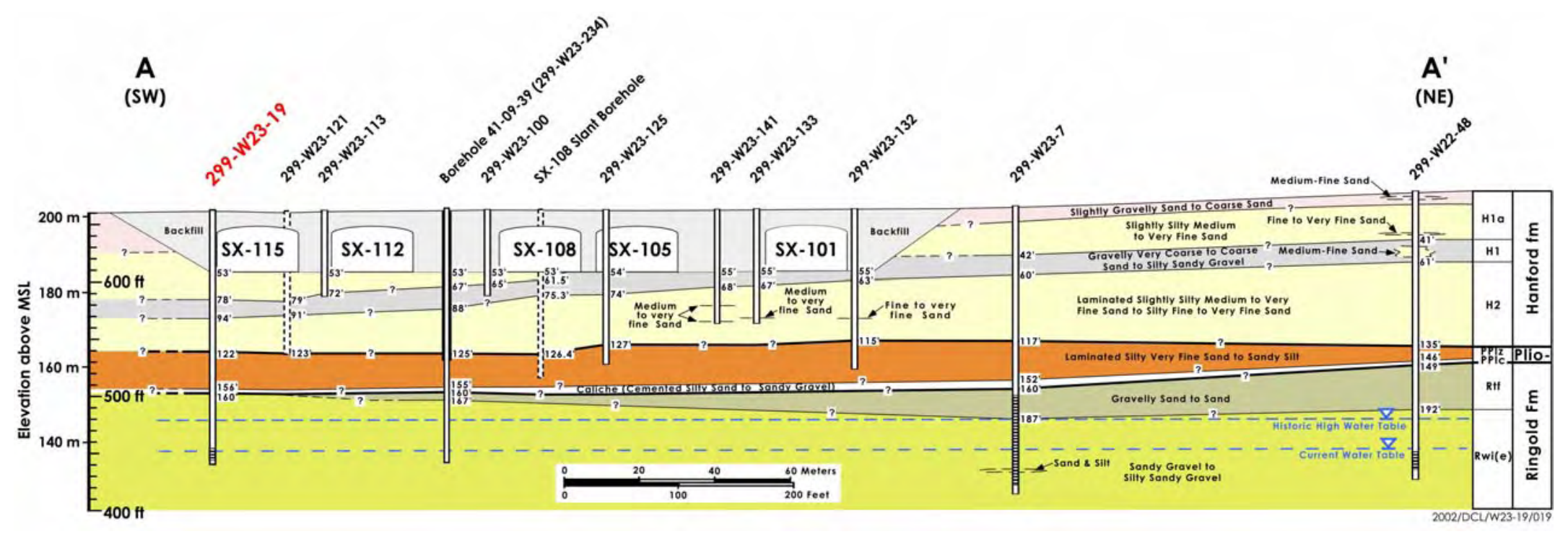

Figure 2.3 Cross Section through 241-SX Tank Farm and Borehole 299-W23-19. 


\subsubsection{Hanford Formation}

Three units of the Hanford formation are in borehole 299-W23-19: (1) an upper fine sand and mud sequence (Hanford formation H1a unit), (2) a middle coarse sand and gravel sequence (Hanford formation $\mathrm{H} 1$ unit), and (3) a lower fine sand and mud sequence (Hanford formation $\mathrm{H} 2$ unit).

Upper Fine Sand and Mud Sequence (Hanford Formation H1a Unit). The Hanford formation H1a unit is between 18.7 meters (61.5 feet) to 23.8 meters (78 feet) depth in borehole 299-W23-19 (Figure 2.2). This sequence consists of several, fining upwards, coarse sand to silty fine sand "rhythmites" deposited during cataclysmic Ice-Age flooding. Mud content within the Hanford formation H1a unit from nearby boreholes is up to 14 wt\% Serne et al. (2002a). Whereas grain sizes within individual beds are gradational, the upper and lower boundaries of the beds are sharp. Higher moisture is usually associated with the fine-grained upper portions of these graded beds, which possess a higher moisture-retention capacity. The $\mathrm{CaCO}_{3}$ content within this unit ranges from 2 to $4 \mathrm{wt} \%$, and generally increased upward toward the top of the unit.

A total of 28 samples was collected from intact cores of the Hanford formation H1a unit for moisture content (see Section 4.0). The moisture content fluctuates significantly within this unit, ranging from a few percent to up to $22 \mathrm{wt} \%$. The higher moisture contents are probably associated with the finer-grained tops of rhythmites. Twelve discrete intervals were analyzed for geochemical characterization from the Hanford formation H1a unit. Geochemical results are discussed in detail in Section 4.0.

Middle Coarse Sand and Gravel Sequence (Hanford H1 Unit). The Hanford formation H1 unit lies at depths between 23.8 meters (78 feet) and 28.7 meters (94 feet) in borehole 299-W23-19 (Figure 2.2). This unit is predominantly a pebbly, medium- to coarse-grained sand (Figure 2.4). At least two layers of fine sand to muddy sand are present within this sequence. Mud content in the coarser-grained facies of the Hanford formation $\mathrm{H} 1$ unit from nearby boreholes is generally low (5 to $7 \mathrm{wt}$. \%) (Table 2.5). The $\mathrm{CaCO}_{3}$ content in this unit is also relatively low $(\sim 2 \mathrm{wt} \%)$.

299-W23-19 (B8809): 85.5' - 86.5'

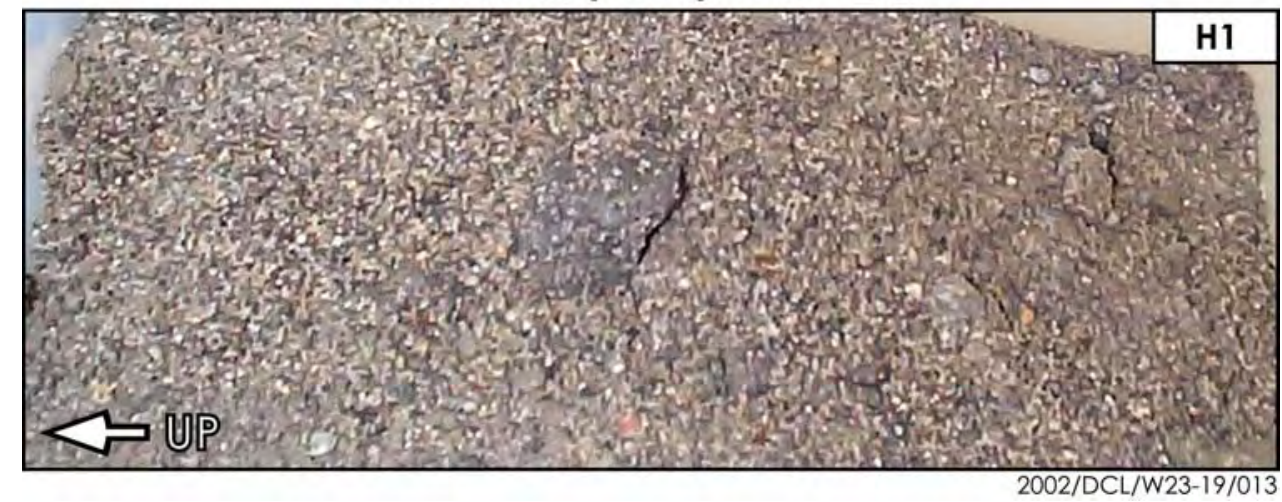

Figure 2.4 Disaggregated core from the Hanford formation H1 unit (85.5-86.6 foot depth) in Borehole 299-W23-19. 
A total of 15 samples were collected from cores in this Hanford formation H1 subunit for moisture content (Figure 2.2). Moisture content is generally only a few wt $\%$ or less, except within finer-grained zones within this unit, which may contain up to $17 \mathrm{wt} \%$ water. Six discrete intervals were analyzed for geochemical characterization from the Hanford formation $\mathrm{H} 1$ unit. Geochemcial results are discussed in detail in Section 4.0.

Lower Fine Sand and Mud Sequence (Hanford Formation H2 Unit). The lower fine sand and mud sequence, which constitutes the Hanford formation $\mathrm{H} 2$ unit in borehole 299-W23-19, lies at depths between 28.7 (94 feet) and 37.2 meters (122 feet) (Figure 2.2). This predominantly fine-grained sequence is composed of mostly stratified fine sand to silty fine sand (Figure 2.5). Several coarser sand interbeds occur toward the top of the sequence. Graded rhythmite development is not as obvious in the Hanford formation $\mathrm{H} 2$ unit compared to the Hanford formation H1a unit. Instead, there appear to be a lot of irregular subtle gradations, mostly between platy (i.e., laminated), silty-fine to very-fine sand and occasionally medium to coarse sand. The $\mathrm{CaC} \mathrm{O}_{3}$ content within this unit is relatively low ( 2 to $3 \mathrm{wt} \%$ ). The geologist's log and the neutron-gamma log (Lindsey et al. 2000) both indicate a zone of higher $\mathrm{CaCO}_{3}$ just below 30 meters (100 feet) depth, in a zone not sampled for laboratory carbonate analysis, which might be associated with a weakly developed paleosol. Based on an increase in background gamma counts on geophysical logs, the base of the Hanford formation $\mathrm{H} 2$ unit is interpreted to lie at approximately 37.2 meters (122 feet) below ground surface in an interval of no core recovery. Thus, the exact nature of the contact between the base of the Hanford formation with the underlying PlioPleistocene unit can not be completely evaluated for this borehole. The 37.2 meters (122 feet) depth chosen for the base of the Hanford formation in this report is very close to the contact between Hanford formation and the Hanford formation/Plio-Pleistocene age deposits selected at 38 meters (124.7 feet) by Lindsey et al. (2000), based on the weakly developed paleosol at this depth.

299-W23-19 (B8809): 112.0' - 112.5'

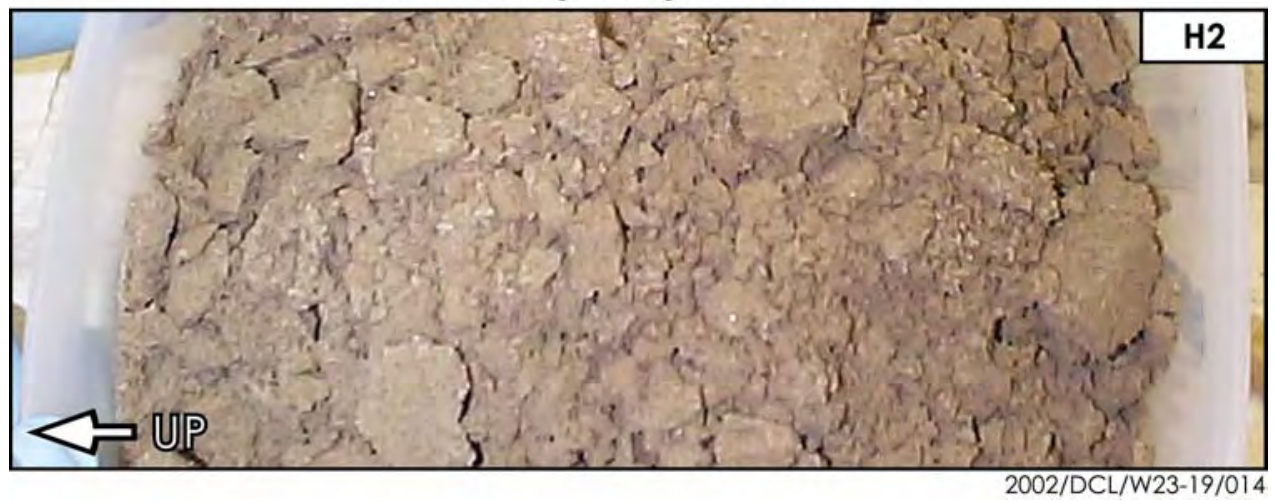

Figure 2.5 Disaggregated Core from the Hanford H2 formation unit (112.0-112.5 foot depth) in Borehole 299-W23-19.

A total of 38 samples were collected from cores in the Hanford formation $\mathrm{H} 2$ subunit for moisture content (see Section 4.0). Moisture content varies widely from a few percent to up to $22 \mathrm{wt} \%$. High moisture contents are probably associated with the finer-grained intervals within this unit. A total of eight discrete intervals were analyzed for geochemcial characterization from the Hanford formation $\mathrm{H} 2$ unit. Geochemcial results are discussed in detail in Section 4.0. 


\subsubsection{Plio-Pleistocene Unit}

The Plio-Pleistocene unit is subdivided into two subunits: (1) the upper Plio-Pleistocene unit (PPlz), characterized by its relatively high silt content, and intercalated nature between silty fine sand and silt/clay; and (2) the lower Plio-Pleistocene unit (PPlc), characterized by secondary, pedogenic calciumcarbonate cement.

Upper Plio-Pleistocene Unit (PPlz). The upper Plio-Pleistocene unit extends from 37.2 meters (122 feet) to 47.5 meters (156 feet) within borehole 299-W23-19 (Figure 2.2). Most of the sediments in the PPlz subunit within borehole 299-W23-19 appear to be fine-grained overbank alluvium, which may include some interspersed, weakly developed paleosols (Figure 2.6). Most beds display well-preserved stratification, except within paleosols where the bedding has been disturbed by blocky soil-ped development, mottling, and/or bioturbation associated with pedogenesis. Dark carbonaceous aggregates (i.e., decomposed organic matter) and secondary iron-oxide and manganese-oxide staining are also common within this subunit. The $\mathrm{CaCO}_{3}$ content is highest ( $\left.\sim \mathrm{wt} \%\right)$ toward the middle of the $\mathrm{PPlz}$ subunit, generally twice as great as that measured for the Hanford formation. The increased $\mathrm{CaCO}_{3}$ content may be the result of more soil development within the PPlz unit or perhaps from the reworking of the underlying, calcium-carbonate-rich PPlc subunit.

299-W23-19 (B8809): 145.1' - 145.6'

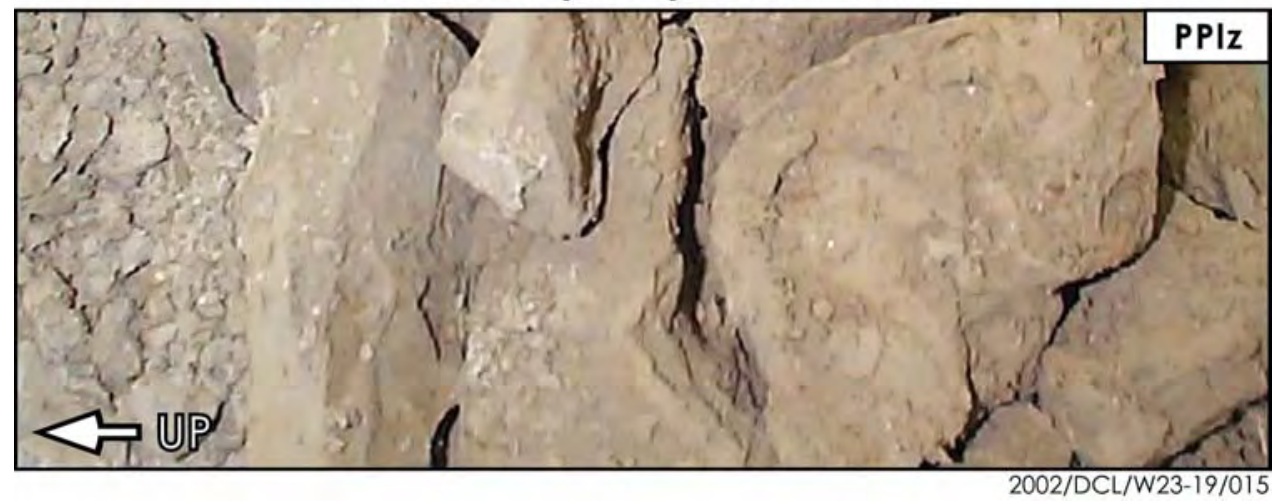

Figure 2.6 Partially Disaggregated Core from the Upper Plio-Pleistocene Unit (145.1145.6 foot depth) in Borehole 200-W23-19.

A total of 62 samples were collected from cores from this subunit for moisture content (see Section 4.0). Moisture content varies widely from a few percent to up to $28 \mathrm{wt} \%$. Moisture collected from the core samples is generally higher for the lower half of the PPlz subunit. The neutron-neutron log, on the other hand, suggests the range of moisture distribution is about the same throughout the subunit. A total of 20 discrete intervals were analyzed for geochemical characterization from the upper Plio-Pleistocene unit. The results from geochemcial analysis presented in this report suggest the upper Plio-Pleistocene unit is one of the more reactive units present in the vadose zone beneath the SX Tank Farm. The PPlz subunit appears to have an affinity for vadose zone groundwater contamination, due to a greater moistureretention capacity and/or more-reactive mineralogy. Retention of contaminants is indicated by the 
relatively high levels of technetium-99 and higher porewater electrical conductivity for this unit. Geochemical results are discussed in detail in Section 4.0.

Lower Plio-Pleistocene Unit (PPlc). The lower Plio-Pleistocene unit extends from 47.5 meters (156 feet) to 48.8 meters (160 feet) within borehole 299-W23-19 (Figure 2.2). The lower Plio-Pleistocene at this location consists of weathered, dark-olive-gray, loose, basaltic, pebbly sand (Figure 2.7), interspersed with more-indurated layers of mud and $\mathrm{CaCO}_{3}$ (Lindsey et al. 2000). Borehole 299-W23-19 appears to be near the boundary between side-stream alluvial facies and massive-carbonate facies of the lower PlioPleistocene unit to the north and east. This is indicated by the highly basaltic and coarse nature of the lower Plio-Pleistocene unit at this location, which is different from other areas within the SX Tank Farm (Serne et al. 2002a, 2002b). Also, unlike other boreholes in the SX Tank Farm, the $\mathrm{CaCO}_{3}$ does not occur as a singular, well-developed caliche layer, but appears to occur as stringers within the coarse-grained, basaltic alluvium. Both the geologist's log and the neutron-gamma geophysical log indicate elevated levels of $\mathrm{CaCO}_{3}$ and calcium ion, respectively (Lindsey et al. 2000).

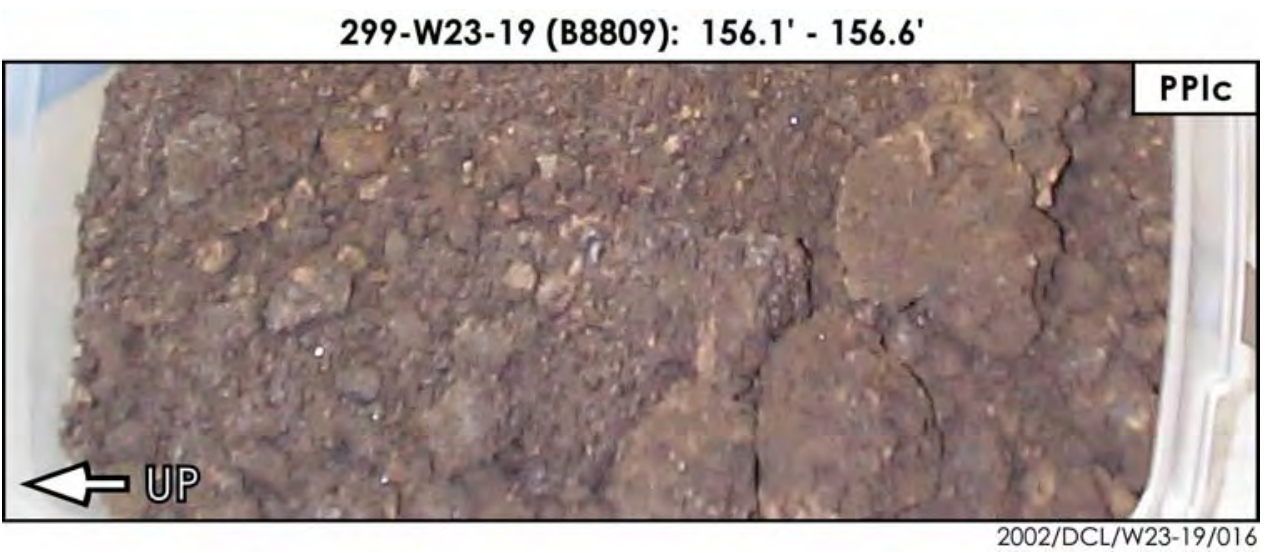

Figure 2.7 Disaggregated Core from the Lower Plio-Pleistocene Unit (156.1-156.6 foot depth) in Borehole 299-W23-19.

A total of four samples were collected from cores in this subunit for moisture content (see Section 4.0). The uppermost sample, from the top of the PPlc subunit, contained about $22 \mathrm{wt} \%$ water, which probably includes some of the silt from the overlying PPlz subunit; the remaining three samples were $<10$ wt $\%$. The neutron-neutron log also shows a sharp decrease in moisture content for the PPlc subunit compared to the overlying PPlz subunit, however, the neutron-neutron log response may have been attenuated by the addition of neutron-absorbing gadolinium to the borehole to evaluate drag down during drilling within this zone (Lindsey et al. 2000).

Two intervals were analyzed for geochemical characterization from the lower Plio-Pleistocene unit. An upper sample contained $<1 \mathrm{wt} \% \mathrm{CaCO}_{3}$, while the second sample (only $25 \mathrm{~cm}$ [0.9 feet] below the upper sample) contained almost $25 \mathrm{wt} \% \mathrm{CaCO}_{3}$. This wide range in $\mathrm{CaCO}_{3}$ content demonstrates the highly variable nature of the PPlc subunit. In summary, besides having a different $\mathrm{CaCO}_{3}$ content, the top of the lower PPLc unit is distinctly different and easily differentiated from the overlying upper PPlz unit, 
based on a sharp drop in the total gamma activity on geophysical logs, as well as a sudden change in texture and mineralogy. Geochemical results are discussed in detail in Section 4.0.

\subsubsection{Ringold Formation}

The remainder of the vadose zone below the 160-foot depth is composed of Ringold Formation, member of Wooded Island, Unit E as described in Lindsey et al. (2000). The recovered materials consist predominately of fluvial, weakly cemented sandy gravel to strongly cemented matrix-supported conglomerate (Figure 2.8). This formation is further characterized by a yellow-brown coloration, and no reaction to hydrochloric acid. The gravel/conglomeratic facies is both clast-supported and matrixsupported. The matrix varies from sandy mud, to muddy fine sand, to well-sorted quartz-rich fine or medium sand, to very coarse sand. The gravel clasts are subrounded to rounded and range up to 70 millimeters ( 3 inches) in diameter. The clasts are composed predominantly of quartzite, basalt, and granite. In places, the basalt and granitic clasts are highly weathered, and iron-oxide staining is common. This formation is extremely difficult to drill with a drive barrel and/or to sample with a split-spoon sampler. Thus, only three split-spoons were recovered from this interval.

299-W23-19 (B8809): 184.2' - 184.9'

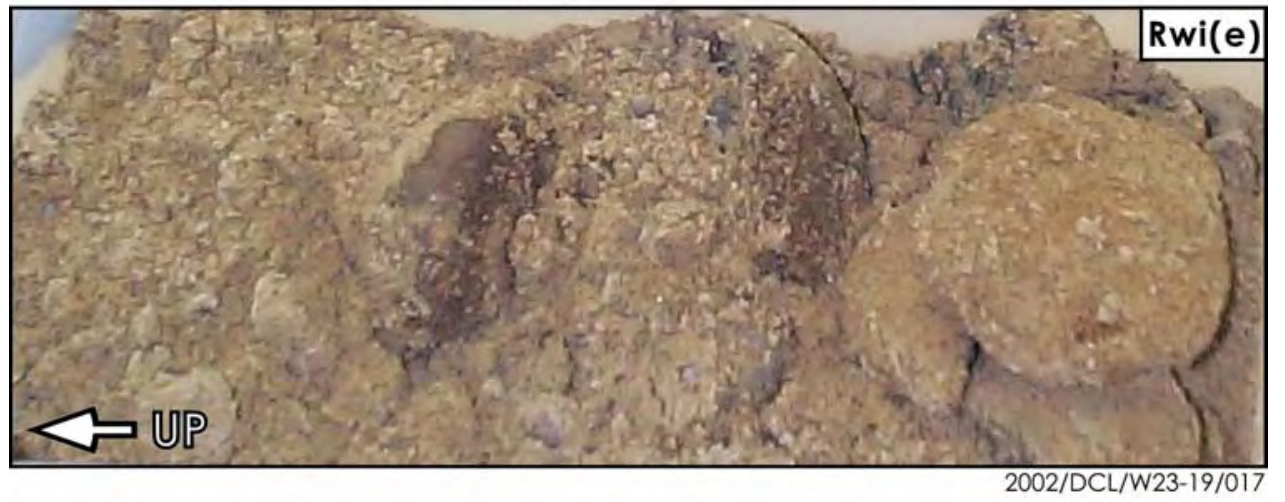

Figure 2.8 Disaggregated Core from Ringold Formation Unit E (184.2-184.9 foot detph) n Borehole 299-W23-19.

A total of five samples were collected from cores from the Ringold Unit E gravels for moisture content (Figure 2.8). These ranged from a few wt $\%$ to $20 \mathrm{wt} \%$ water. A total of 10 intervals were analyzed for geochemical characterization from Ringold Unit E. In general, the Ringold Formation appears to be relatively nonreactive, geochemically, when compared to the other overlying stratigraphic units. Geochemical results are discussed in detail in Section 4.0. 


\subsection{Historical Water Levels}

Figure 2.9 illustrates hydrographs for wells $299-$ W23-3 and -4. Since a complete water level record is unavailable for either well, their water level behavior was extrapolated from each other to complete the record. This is justified by the similar behaviors of their common data sets. Based on this extrapolation between the two data sets, and linear interpolation over the distance between the two wells, it is estimated that the peak water elevation beneath the SX-115 tank was approximately 146 meters (479 feet) above mean sea level. This occurred in 1976 and places the water table approximately 55 to 56 meters (182 feet) bgs or 40 meters (132 feet) beneath the bottom of the tank. A secondary maximum occurred in 1984, just before the 216-U-10 Pond was decommissioned. At this time, the water table was estimated to have been almost as high as it was in 1976. The water table was encountered at a depth of approximately 64.5 meters (212 feet) in borehole 299-W23-19 in August 1999. Thus, the water table has dropped an estimated 8 to 9 meters (28.5 feet) over the last 14 years. An examination of the hydrographs since about 1988 suggests that the water level is dropping at a rate of 0.5 to 0.6 meters per year ( 1.5 to 1.9 feet per year).

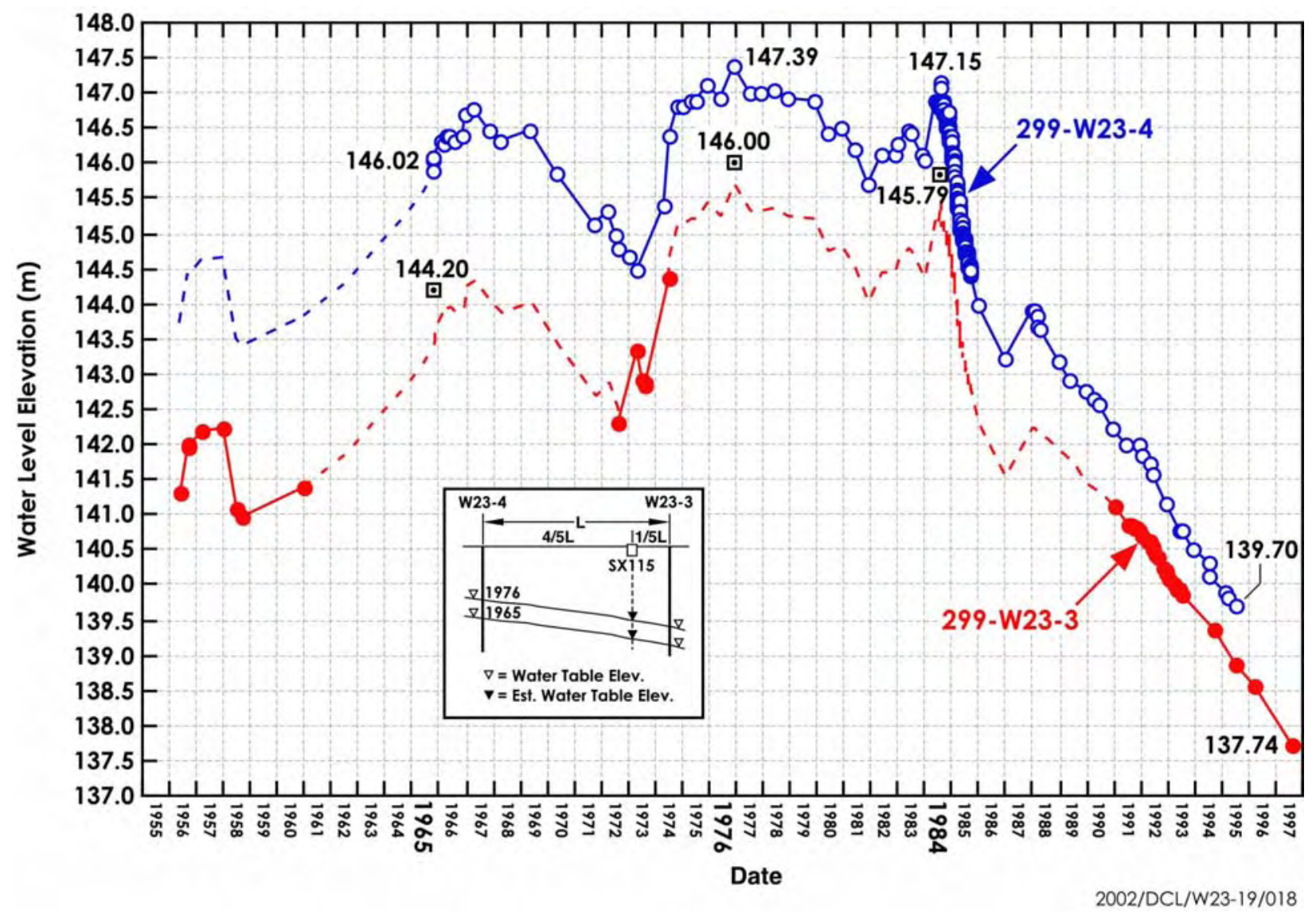

Figure 2.9 Historic Water Levels Beneath the 241-SX Tank Fram. 
The water table in 1965, when tank SX-115 was determined to be a leaker and was removed from service, is estimated to have been at about 144 meters ( 472 feet) elevations, or 57 to 58 meters (189 feet) bgs. Based on HNF-EP-0182, Rev. 153, SX-115 leaked 50,000 gallons of fluid (condensate plus dissolved salt cake [mostly sodium nitrate]) in March 1965. Within days of discovering the leak, the remaining liquid was pumped to tank SX-105 and the tank dried from self-heating. In 1988 the interior of the tank was probed and photographed. The 1988 monitoring led to the conclusion that there was $\sim 12,000$ gallons of dry sludge in the bottom of the tank. 


\subsection{Geochemical Method and Materials}

This chapter discusses the decisions used to determine which samples would be characterized, what parameters would be measured, and the laboratory methods used to obtain those parameters.

\subsection{Sample Inventory}

Split-spoon sleeves were sent directly to the Pacific Northwest National Laboratory (PNNL) chemical laboratory in the 3720 Building after they were removed from the core barrel, and end caps were placed on them to preserve the moisture content and to keep the sample intact. Laboratory support to the SX-115 coring started in August 1999. In all, 257 samples were received and analyzed for a variety of geochemical, radiochemical, and chemical constituents. The samples included 239 split-spoon sleeve samples (211 samples were 6 inches in length and 4 inches in diameter and 28 samples were 13 inches in length and 4 inches in diameter), 9 polyethylene bottles containing material cleaned out of or sloughed down the borehole, and 9 "socks" containing sediment collected from the sampling cyclone during airrotary drilling in the Ringold Formation.

Samples were identified by the borehole number, B8809, followed by a suffix such as -01 , which represents a specific split-spoon. Most sampling events collected a 5-foot split-spoon. Individual sleeves within a split-spoon were identified by the letters A through $\mathrm{K}$, where the A sleeve was always in the position closest to the drive shoe (the deepest) and subsequent letters representing sequentially shallower sleeves within the split-spoon. Thus, the K sleeve would contain the first sediment and the A sleeve would contain the last sediment collected in the sampler during each 5-foot sampling event. In several instances, less than 5 feet of sample were collected when the drive barrell met resistance. Chip samples collected from the sampling cyclone were designated with an "S" before a sleeve identifier.

\subsection{Sampling}

During the drilling of borehole 41-09-39, changes in sediment type and contaminant concentration were noted within a few inches in a given sleeve. This led to the conclusion that a more methodical approach would be necessary to provide the technical justification for selecting samples for detailed characterization from borehole 299-W23-19, as defined in the data quality objectives process. Subsequently, a tiered methodology was developed that considered sample depth, geology (e.g., lithology, grain-size composition, carbonate content), contaminant type and concentration (e.g., radionuclides, nitrate), moisture content, and overall sample quality during sample collection. Inexpensive analyses and certain key parameters (i.e., moisture content, gamma energy analysis) were performed on sediment from each sleeve.

The goal of the characterization was to quantify the extent that mobile contaminants had penetrated into the vadose zone sediment. Immediately following the geologic examination, the sleeve contents were subsampled for moisture content, gamma-emission radiocounting (effectively cesium-137, potassium-40, uranium-238, and thorium-232 data), 1:1 water extracts (which provide soil $\mathrm{pH}$, electrical conductivity, cation, and anion data), and soil tension. Originally, about 30 sleeve and "sock" samples 
were selected based on high moisture content and proximity to major geologic contacts. After preliminary data were available, additional samples were selected to fill out a depth profile for electrical conductivity, chromium(VI), and technetium-99.

Each sleeve was weighed, measured, opened in a radiologically controlled laboratory space, and placed in a sealable plastic container. The samples were photographed and examined using standard geologic techniques. The geologic examination included the following: fullness of the sleeve, grain size, moisture, color, and qualitative carbonate determination. Drilling records and daily logs were used to assess actual sample depth to compare with the sampling field records.

Analyses performed on samples from borehole 299-W23-19 were not performed with a bias for sleeves with lower letters (i.e., deeper) as was done for other boreholes in the SX Tank Farm because no significant cesium-137 contamination was found in the samples. For other boreholes with significant cesium-137 contamination (e.g., borehole 41-09-39), drag-down effects were noted such that the deepest sleeves showed the least drag-down effects. No measurable drag-down effects were noted for mobile contaminants that are concentrated mainly in the porewater and not on sediment sorption sites.

After sampling was completed, the remaining sediment from each sleeve was sealed and placed in cold storage. The sleeve, bag, tape, and any other associated packaging material were weighed so that net weight of the sediment could be estimated. The packaging materials then were disposed. All information was recorded in laboratory record books.

\subsection{Materials And Methods}

Every effort was made to minimize moisture loss and prevent cross contamination between sleeve samples during laboratory subsampling. Depending on the sample matrix, very coarse pebble and larger material (>32 millimeters) was excluded from subsamples so that the moisture content was similar to that of the radionuclide counting sample and the 1:1 sediment-to-water extract sample. For this reason, the interpretation of measurement results from sub-samples should consider possible biases toward larger concentrations of analytes considered to be associated with smaller-size fractions. Sediment collected above the Ringold Formation contained no large pebbles, cobbles, or boulders.

The procedures used to visually describe the samples were ASTM D 2488 (ASTM 1993) and PNLDO-1 (PNL 1990). The sediment classification scheme used for geologic description of sediment types was based on a modified Folk/Wentworth classification scheme described in Fecht and Price (1977). However, the mineralogic and geochemical characterization required further separation of the "mud" fraction of the Folk/Wentworth classification into silt and clay-size fractions.

\subsubsection{Moisture Content}

Gravimetric water content was determined using PNNL Procedure PNL-MA-567-SA-7 (PNL 1990). That procedure is based on the American Society for Testing and Materials procedure Laboratory Determination of Water (Moisture) Content of Soil, Rock, and Soil-Aggregate Mixtures (procedure 
ASTM D2216 in ASTM 1986). A 30- to 80-gram subsample was taken from each sleeve after mixing the entire sample in a sealable plastic container. Moisture samples were placed in tared containers, weighed, and oven dried at $105^{\circ} \mathrm{C}$ for about 24 hours until constant weight was achieved. The containers then were removed from the oven, sealed, cooled, and weighed. The gravimetric water content was computed as percentage change in soil weight before and after oven drying. A calibrated balance was used for all weightings and a calibrated weight set was used to verify balance performance.

\subsubsection{1:1 Sediment-to-Water Extract}

One of the objectives of placing borehole 299-W23-19 near the southwest corner of the SX Tank Farm was to determine whether mobile contaminants, such as chromium(VI), technetium-99, and nitrate were reaching the groundwater in that vicinity. The results of the investigation at borehole 41-09-39 (Serne et al. 2002b) and analyses of historical gross gamma logs (Sobczyck 2000; Johnson and Chou 1998; Randle and Price 1999) from dry wells in the tank farm suggested that the vadose zone sediment dipped toward the southwest. Also, field screening for radionuclides indicated that the samples from borehole 299-W23-19 did not contain significant amounts of gamma emitters so attention was focused on measuring the mobile contaminants. The most economical method of determining the subsurface distribution of chromium(VI), technetium-99, and nitrate is to use the water extracts.

The concentrations of soluble inorganic constituents were determined using 1:1 sediment to deionized water extract method. This method was chosen because the sediment was too dry to easily extract sufficient quantities of vadose zone porewater for analysis. The extracts were prepared by adding enough weight of deionized water to approximately 70 to 100 grams of sediment such that the amount of deionized water added plus existing in a porewater equaled the weight of over dry sediment. The deionized water was added to a screw-cap jar containing sediment sample and the jar was sealed, briefly shaken by hand, then placed on a mechanical orbital shaker for 1 hour. The resulting slurry was allowed to settle until the supernatant liquid was fairly clear. The supernatant was carefully decanted and separated into unfiltered aliquots for measurements of electrical conductivity and $\mathrm{pH}$, and filtered aliquots (passed through $0.45 \mu \mathrm{m}$ membrane) for anion, cation, carbon, and radionuclide analyses. More details can be found in Rhoades in ASA (1996a).

\subsubsection{1 pH and Electrical Conductivity.}

Two approximately 3 milliliter aliquots of unfiltered, 1:1 sediment-to-water extract supernatant were used for $\mathrm{pH}$ and electrical conductivity measurements. The $\mathrm{pH}$ of extracts was measured with a solidstate Ross electrode and a pH meter calibrated with $\mathrm{pH}$ buffers of 4, 7, and 10. Electrical conductivity was measured and compared to potassium chloride standards within the range of $0.001 \mathrm{M}$ to $1.0 \mathrm{M}$.

\subsubsection{Anions}

Aliquots of 1:1 sediment-to-water extract were analyzed for anion concentrations using an ion chromatograph. Fluoride, chloride, nitrite, phosphate, sulfate, and oxalate were separated on a Dionex AS4A column with a $1.75 \mathrm{mM} \mathrm{NaHCO} / 1.85 \mathrm{mM} \mathrm{Na}_{2} \mathrm{CO}_{3}$ eluent and measured using a conductivity detector according to procedure, which is based on U.S. Environmental Protection Agency (EPA) Method 300.0A (EPA-600/R-93-100). 


\subsubsection{Cations and Trace Metals}

Concentrations of major cations were determined by the inductively coupled plasma (ICP) method, atomic emission spectrometry with high-purity calibration standards to generate calibration curves and to verify continuing calibration during the analysis. Dilutions of 100x, 50x, 10x, and 5x were made from each sample to investigate and correct for matrix interferences. Details are found in U.S. Environmental Protection Agency (EPA) Method 6010A (SW-846).

Inductively coupled plasma-mass spectrometry (ICP-MS) was used to determine trace metal concentrations, including technetium-99 and uranium-238, according to procedure PNNL-AGG-415 (PNNL 1998). This method is similar to EPA Method 6020 (EPA 2000).

\subsubsection{Alkalinity and Carbon}

The alkalinity was measured for several 1:1 sediment-to-water extracts using a standard titration method equivalent to ASTM Method D3875-97 (ASTM 1997). Inorganic and organic carbon were determined with a carbon analyzer using procedure ASTM Method D4129-82 "Standard Test Method for Total and Organic Carbon in Water: Oxidation by Coulometric Detection” (ASTM 1982).

\subsubsection{Porewater Composition}

Selected samples were packed in drainable cells that were inserted into an ultracentrifuge. The samples were centrifuged for up to 8 hours at several thousand $\mathrm{m} / \mathrm{sec} / \mathrm{sec}$ to "squeeze" the pore water out of the sediment. $\mathrm{pH}$, electrical conductivity and cation, trace metal, and anion concentrations were measured on the porewater using the same techniques as those used for the 1:1 sediment-to-water extracts.

\subsubsection{Radionuclide Analysis}

\subsubsection{Gamma Energy Analysis}

Gamma energy analysis was performed only on selected samples because no gamma activity above background was found in the initial samples analyzed. Analyses were done on samples from right above and below each geologic contact and samples with high moisture contents. No gamma energy analysis was performed on water or acid extracts of contaminated sediment after it was determined that no elevated gamma activity was in the borehole sediment.

All gamma energy analyses used $60 \%$ efficient, intrinsic germanium gamma detectors. All germanium counters were efficiency calibrated for distinct geometries using National Institute of Standards and Technology (NIST)-traceable mixed gamma standards. Samples analyzed were 500 to 700 grams in a $500 \mathrm{~cm}^{3}$ fixed geometry. All spectra were background corrected. Spectral analyses used libraries containing most mixed fission products, activation products, and natural decay products. Control samples were run throughout the analysis to ensure correct operation of the detectors. The control samples contained isotopes with photo peaks spanning the full detector range and were monitored for peak position, counting rate, and full-width at half-maximum. Details are found in PNNL-RRL-01, “ Gamma Energy Analysis, Operation, and Instrument Verification using Genie2000 Support Software” (PNNL 1997). 


\subsubsection{Strontium-90}

Sediment for strontium-90 analysis was weighed and spiked with strontium-85. Samples were leached overnight with concentrated nitric acid after which an aliquot of the leachate was diluted 50\% with deionized water. The resulting solutions were passed through SrSpec columns Eichrome with $8 \mathrm{M}$ nitric acid to capture strontium. The column resins then were washed with 10 column volumes of $8 \mathrm{M}$ nitric acid. The strontium was eluted from the SrSpec column using deionized water. The extract was evaporated to dryness in a liquid scintillation vial and was ready for counting after adding the cocktail. The purified strontium samples were analyzed first by gamma spectroscopy to determine chemical yield from the added tracer and to quantify any contamination from cesium-137. Samples then were analyzed by liquid scintillation counting to determine the amount of strontium-90. A matrix spike, a blank spike, a duplicate, and blanks were run with each sample set to determine the efficiency of the separation procedure as well as the purity of reagents. Chemical yields were generally good with some explainable exceptions. Matrix and blank spike yields were good, bias was consistent, and blanks were below detection limits.

\subsubsection{Tritium}

The tritium content of selected sediment samples was determined by distillation from 1-gram samples using PNNL method PNL-ALO-418 (1994) and a Lachat Microdistillation apparatus. The tritium was condensed on special GORE-TEX collectors that then were analyzed by liquid scintillation.

\subsubsection{Carbon Content}

The carbon content was determined by ASTM Method D4129-82, A Standard Test Method for Total and Organic Carbon in Water Oxidation by Coulometric Detection (ASTM 1982). Total carbon in all samples was determined with a Coulometrics Inc. Model 5051, Carbon Dioxide Coulometer with combustion at approximately $980^{\circ} \mathrm{C}$. Ultrapure oxygen was used to sweep the combustion products through a barium chromate catalyst tube for conversion to carbon dioxide. Samples were placed into precombusted, tared, platinum combustion boats and weighed on a four-place analytical balance. After the combustion boats were placed into the furnace introduction tube, a 1-minute waiting period was allowed so that the ultrapure oxygen carrier gas could remove any atmospheric carbon dioxide introduced to the system during sample placement. After this system purge, the sample was moved into the combustion furnace. Evolved carbon dioxide was quantified through coulometric titration following absorption in a solution containing ethanolamine. Sample titration readings were performed at 3 minutes after combustion began and again once stability was reached, usually within the next 2 minutes. The system background was determined by performing the entire process using an empty, pre-combusted platinum boat. Adequate system performance was confirmed by analyzing known quantities of a calcium carbonate standard.

Inorganic carbon contents were determined using a Coulometrics, Inc., Model 5051 Carbon Dioxide Coulometer. Soil samples were weighed on a four-place analytical balance, then placed into acid-treated glass tubes. The sample tubes were placed into the system and a 1-minute waiting period allowed the ultrapure oxygen carrier gas to remove any introduced atmospheric carbon dioxide. Inorganic carbon was released through acid-assisted evolution (50\% hydrochloric acid) with heating to $200^{\circ} \mathrm{C}$. Samples were 
completely covered by the acid to allow full reaction to occur. Ultrapure oxygen gas swept the resultant carbon dioxide through the equipment to determine inorganic carbon content by coulometric titration. Sample titration readings were performed at 5 minutes following acid addition and again once stability was reached, usually within 10 minutes. Known quantities of calcium carbonate standards were analyzed to verify that the equipment was operating properly. Background values were determined.

Organic carbon was calculated by subtracting the amount of inorganic carbon from total carbon.

\subsubsection{M Nitric Acid Extract}

$8 \mathrm{M}$ nitric acid extraction is a protocol used by EPA to estimate the maximum concentrations of regulated metals in contaminated sediment that would be biologically available. We subjected aliquots of contaminated sediment from 299-W23-19 to the acid extraction to search for obvious signs of elevated concentrations of chemically reactive elements from leaked tank fluids.

Approximately 20 grams of oven-dried sediment were contacted with $8 \mathrm{M}$ nitric acid at a ratio of $\sim 5$ parts acid to 1 part sediment. The slurries were heated to $\sim 80^{\circ} \mathrm{C}$ for several hours and then the fluid was separated by centrifugation and filtration through $0.2 \mu \mathrm{m}$ membranes. The acid extracts were analyzed for major cations and trace metals using ICP and ICP-MS, respectively. The acid digestion procedure is based on EPA Method 3050B (SW-846).

\subsubsection{Elemental Analysis}

The elemental composition of the bulk sediment was determined by a combination of energy and wavelength dispersive $x$-ray fluorescence using methods developed at PNNL. Energy dispersive x-ray fluorescence analyses utilized a KEVEX 0810A commercial $\mathrm{x}$-ray fluorescence excitation and detection subsystem. Samples were first homogenized in a Coors high-density alumina $\left(\mathrm{Al}_{2} \mathrm{O}_{3}\right)$ mortar and pestle. Six hundred milligrams of the mixed sample were removed and ground a second time to $\sim 300$ mesh. Samples then were placed between two sheets of stretched para-film and loaded into the 0810A x-ray fluorescence unit. Count acquisition times ranged from 600 to 3000 seconds, depending on the targets (gadolinium, iron, silver, and zirconium). Concentrations of forty-one elements (aluminum, antimony, arsenic, barium, bromine, cadmium, calcium, cerium, cesium, chlorine, chromium, copper, gallium, iodine, indium, iron, lanthanum, lead, manganese, molybdenum, nickel, niobium, palladium, potassium, phosphorous, rhodium, rubidium, ruthenium, selenium, silicon, silver, strontium, sulfur, tellurium, thorium, tin, titanium, uranium, vanadium, yittrium, and zinc) were obtained from each sample. Spectrum interpretation was by the backscatter fundamental parameter approach (described in PNNL 2000a).

Wavelength dispersive x-ray fluorescence analysis was done with a Siemens Spectra 3000 instrument, equipped with both a flow detector to detect soft radiation from low $\mathrm{Z}$ elements, and a scintillation detector for the more energetic, high $\mathrm{Z}$ element radiation. Bulk, solid samples were prepared by grinding 180 to 1500 milligrams of sample to 300 mesh and pressing it into a 3.2-centimeter-diameter pellet, using a 27,000-kilogram laboratory press. Standard addition and similar matrix methods were used to generate calibration curves for sodium and magnesium, which were then used to process the data. Additional discussion of $\mathrm{x}$-ray fluorescence techniques for quantitative analysis of sediment are found in 
Chapter 7 Elemental Analysis by X-Ray Fluorescence Spectroscopy, Part 3, pages 161-223 (ASA 1996) and in the Siemens Spectra 3000 Reference Manual (1994).

\subsubsection{Particle Size Distribution}

Both dry sieving and wet sieving/hydrometer methods were used to determine particle-size distributions. For the dry sieving method, 120 to 210 grams of oven-dried sediment were sieved through a sequence of $4,2,1,0.5,0.25,0.212,0.125,0.063$ millimeter sieves onto a pan. An auto shaker, either a Rotap Model RX-29 or a Gilson Model SS-8R, was used.

The wet sieve/hydrometer method (ASA [1986a], Part 1; Chapter 15-5 Hydrometer Method [pages 404-408]) also was used to quantify particle-size distributions and concentrated on quantifying the silt and clay fractions. The silt and clay separates were saved for mineralogical analyses. Samples used for the hydrometer method were never air or oven dried to minimize the effects of particle aggregation leading to results that are biased toward coarser material.

\subsubsection{Particle Density}

Particle, or grain, density was determined with pychnometers (see ASA 1986b, Part 1, Chapter 14-3 Pychnometer Method) using oven-dried material. The particle density is needed to determine particle size from hydrometer data.

\subsubsection{Mineralogy}

The mineralogy of the bulk sample, the silt-size fraction, and the clay-size fraction of selected samples was determined by x-ray diffraction techniques. One hundred grams of bulk sample was dispersed in 1.0 liter of a $0.001 \mathrm{M}$ solution of sodium hexametaphosphate. The suspensions were allowed to shake overnight to ensure complete dispersion. The sand fraction was separated from the dispersed sample by wet sieving through a \# 230 sieve. The silt fraction was separated from the clay fraction by settling according to Stoke's law (Jackson 1969). The lower limit of the silt fraction was taken at 2 microns. The sand and silt fractions were oven dried at $110^{\circ} \mathrm{C}$ and prepared for $\mathrm{x}$-ray diffraction and $\mathrm{x}-$ ray fluorescence analyses.

The bulk samples and coarser size fractions were prepared for x-ray diffraction analysis by crushing approximately 0.5 grams of air-dried sample to a fine powder that was packed into a small, circular holder.

Each clay suspension was concentrated to approximate 10 milliliters by adding a few drops of $10 \mathrm{~N}$ magnesium chloride to the dispersing solution. The amount of total clay in the concentrated suspensions was determined by drying known volumes and weighing the dried sediment. The density of the slurry was calculated from the volume pipetted and the final weight. Volumes of slurry equaling 250 milligrams of clay were transferred into centrifuge tubes and treated to remove carbonates following Jackson (1969). The carbonate-free clay fraction then was saturated with either magnesium (II) or potassium cations. Clay samples were mounted for x-ray diffraction analyses by the method described in Drever (1973) and 
placed onto an aluminum slide. Potassium saturated slides were air dried and analyzed, then heated to $575^{\circ} \mathrm{C}$ and reanalyzed.

All samples were analyzed on a Scintag diffractometer equipped with a Pelter thermoelectrically cooled detector and a copper $x$-ray tube. Slides of preferentially oriented clay were scanned from 2 to $45^{\circ}$ $2 \theta$, and randomly oriented powder mounts of bulk sample were scanned from 2 to $75^{\circ} 2 \theta$.

Semiquantitative determination of mineral phases by $\mathrm{x}$-ray diffraction was preformed according to Brindley and Brown (1980).

In addition to x-ray diffraction, transmission electron microscopy (TEM) characterization of selected samples was done with a JEOL 1200X electron microscope equipped with a Links detector system. Samples were prepared for TEM by transferring a small aliquot of dilute clay slurry onto a formvar, carbon-coated, 3-millimeter, copper support grid. The clay solution contained $0.15 \%$ tert-butylamine to reduce the surface tension of water.

Structural formulas were derived from data collected by the TEM analysis. On average, energy dispersive $\mathrm{x}$-ray spectra of clay minerals were collected from a minimum of five particles from the same mineral phase common to the sample. The x-ray spectra were collected and processed using the CliffLorimer Ratio Thin Section method and then converted to a structural formula (based on half-unit cell $\left(\mathrm{O}_{10}(\mathrm{OH})_{2}\right.$ for phyllosilicales) by the method described in Moore and Reynolds (1989).

\subsubsection{Water Potential (Suction) Measurements}

Suction measurements were made on selected sediment from the borehole using the filter paper method PNL-MA-567-SFA-2 (PNL 1990). This method relies on the use of filter paper that rapidly equilibrates with the sediment sample. At equilibrium, the matric suction in the filter paper is the same as the matric suction of the sediment sample. Dry filter papers are placed in an airtight container with the sediment for at least 1 week to allow sufficient time for the matric suction in the sediment to equilibrate with the matric suction in the filter paper. The mass of the wetted filter paper is subsequently determined, and the suction of the sediment is determined from a calibration relationship between filter paper water content and matric suction.

The relationships used for converting the water content of filter paper to matric suction for Whatman \#42 filter paper have been determined by Deka et al. (1995) and can be expressed as:

$$
\begin{aligned}
& \mathrm{Sm}=10^{(5.144-6.699 \mathrm{w})} / 10 \text { for } \mathrm{w}<0.5 \\
& \mathrm{Sm}=10^{(2.383-1.309 \mathrm{w})} / 10 \text { for } \mathrm{w}>0.5
\end{aligned}
$$

where: $\mathrm{Sm}=$ the matric suction $(\mathrm{m})$ and

$\mathrm{w}=$ the gravimetric water content $(\mathrm{g} / \mathrm{g})$

Nine samples from borehole 299-W23-19 were analyzed for water content and soil matric suction. 


\subsubsection{Semi-Volatile Organic Analyses}

Regulators from the Washington State Department of Ecology expressed strong interest in having some of the sediment samples from borehole 299-W23-19 characterized for regulated organic

constituents. It was agreed that the most likely candidates would be semi-volatile organics that may have survived the self-boiling tank environment and several years existence in the vadose zone. Therefore, six samples from borehole 299-W23-19 were analyzed for volatile organic compounds. One sample was from the backfill above the tank to serve as a background sample and five samples were from the base of geologic contacts or in the finer-grained sediments within the Hanford and Plio-Pliestocene formations. Depths of the 5 sediment samples were 22.8 meters ( 74.4 feet), 38.1 meters ( 125 feet), 42.1 meters (138 feet), 45.3 meters (148.5 feet), and 47.2 meters ( 154.7 feet). 


\subsection{Results And Discussion}

This section presents the geochemical, physical, and hydrologic characterization data collected from selected samples from borehole 299-W23-19 and from materials collected in the "socks" filled by the airrotary cyclone sampler. The analytical tests emphasized those that were inexpensive or that were key to determining the vertical distribution of contaminants. Data presented in this section include results of analyses of moisture content, $\mathrm{pH}$, electrical conductivity, and major cations, anions, and radionuclides. Also, the particle-size distribution and mineralogy of selected samples were determined to help select contacts between major geologic units and to help discern geochemical and mineralogic changes caused by the interactions of sediment with tank leak fluids. Finally, matric potential of sediment from selected sleeves was measured to determine whether sufficient moisture was present in the sediment to promote active draining.

\subsection{Moisture Content}

Table 4.1 gives the moisture content of each sample. Figure 4.1 shows the moisture content versus depth. The plot shows very low moisture content in the backfill materials. Moisture content increases in the H1a unit below the bottom of the tank and varies between about 5 and $25 \mathrm{wt} \%$ from the bottom of the tank to the top of the upper Plio-Pleistocene unit at about 47.5 meters (156 feet) below ground surface (bgs). There is a slight tendency for the average moisture content to increase through the Hanford formation $\mathrm{H} 2$ unit and the upper Plio-Pleistocene unit. An abrupt decrease in moisture from about 20 to $25 \mathrm{wt} \%$ to less than $10 \mathrm{wt} \%$ occurs in the lower Plio-Pleistocene unit. In contrast, typical moisture contents for the Hanford formation are generally less than $10 \mathrm{wt} \%$ with some thin zones having up to 15 to $20 \mathrm{wt} \%$ moisture (Serne et al. 2002a). Typical upper Plio-Pleistocene sediment generally has between 5 and $15 \mathrm{wt} \%$ moisture. Comparing the moisture content in borehole 299-W23-19 with that of uncontaminated boreholes, such as 299-W22-48 and 299-W22-50 (Serne et al. 2002a), indicates that there is anomalously high moisture in much of the Hanford formation and the upper Plio-Pleistocene unit. Hard-tool drilling was initiated at the top of the Ringold Formation 56 meters (161 feet bgs) so that the moisture content in the Ringold Formation may reflect water added during drilling.

Both the laboratory generated moisture data and the geophysical neutron log are shown in Figure 4.1. The laboratory data and the geophysical log were collected in different units (weight versus volume). However, there is a general qualitative agreement between the two. Both show increased moisture content in the Hanford formation $\mathrm{H} 2$ unit and the upper Plio-Pleistocene unit relative to the overlying and underlying sediment. 
Table 4.1 Moisture Content of Sediment from Borehole 299-W23-19

\begin{tabular}{|c|c|c|c|c|c|c|c|c|c|c|c|c|c|c|c|c|c|}
\hline \begin{tabular}{|c} 
Sample \\
ID
\end{tabular} & \begin{tabular}{|c|} 
Depth \\
(ft \\
bgs)
\end{tabular} & \begin{tabular}{|c|} 
\\
Wt $\%$ \\
Moisture
\end{tabular} & $\begin{array}{c}\text { Sample } \\
\text { ID }\end{array}$ & \begin{tabular}{|c|} 
Depth \\
(ft \\
bgs)
\end{tabular} & $\begin{array}{c}\text { Wt\% } \\
\text { Moisture }\end{array}$ & $\begin{array}{c}\text { Sample } \\
\text { ID }\end{array}$ & $\begin{array}{c}\text { Depth } \\
\text { (ft } \\
\text { bgs) }\end{array}$ & $\begin{array}{c}\text { Wt\% } \\
\text { Moisture }\end{array}$ & $\begin{array}{c}\text { Sample } \\
\text { ID }\end{array}$ & $\begin{array}{c}\text { Depth } \\
\text { (ft } \\
\text { bgs) }\end{array}$ & \begin{tabular}{c|} 
Wt $\%$ \\
Moisture
\end{tabular} & $\begin{array}{c}\text { Sample } \\
\text { ID }\end{array}$ & $\begin{array}{c}\text { Depth } \\
\text { (ft } \\
\text { bgs) }\end{array}$ & $\begin{array}{c}\text { Wt } \% \\
\text { Moisture }\end{array}$ & $\begin{array}{c}\text { Sample } \\
\text { ID }\end{array}$ & $\begin{array}{c}\text { Depth } \\
\text { (ft } \\
\text { bgs) }\end{array}$ & Moisture \\
\hline $01 \mathrm{~F}$ & 6.55 & 6.80 & $10 \mathrm{C}$ & 36.35 & 9.42 & $18 \mathrm{E}$ & 69.45 & 9.81 & $26 \mathrm{E}$ & 102.95 & 7.04 & $31 \mathrm{C}$ & 131.15 & 8.98 & $38 \mathrm{~F}$ & 152.60 & 13.47 \\
\hline $01 \mathrm{E}$ & 7.05 & 4.96 & $10 \mathrm{~B}$ & 36.85 & 9.15 & $18 \mathrm{D}$ & 69.95 & 12.32 & $26 \mathrm{D}$ & 103.45 & 3.79 & $31 \mathrm{~B}$ & 131.65 & 5.43 & $38 \mathrm{E}$ & 153.10 & 14.07 \\
\hline 01D & 7.55 & 5.79 & $10 \mathrm{~A}$ & 37.35 & 8.38 & $18 \mathrm{C}$ & 70.45 & 15.66 & $26 \mathrm{C}$ & 103.95 & 6.99 & $31 \mathrm{~A}$ & 132.15 & 16.92 & $38 \mathrm{D}$ & 153.60 & 15.96 \\
\hline $01 \mathrm{C}$ & 7.86 & 6.89 & $11 \mathrm{~F}$ & 40.85 & 6.20 & $18 \mathrm{~B}$ & 70.95 & 14.86 & $26 \mathrm{~B}$ & 104.45 & 8.99 & $32 \mathrm{G}$ & 132.75 & 4.97 & $38 \mathrm{C}$ & 154.10 & 17.95 \\
\hline $01 \mathrm{~B}$ & 8.34 & 4.41 & $11 \mathrm{E}$ & 41.35 & 6.51 & $18 \mathrm{~A}$ & 71.45 & 19.61 & $26 \mathrm{~A}$ & 104.95 & 6.81 & $32 \mathrm{~F}$ & 133.25 & 5.47 & $38 \mathrm{~B}$ & 154.60 & 19.15 \\
\hline $01 \mathrm{~A}$ & 9.54 & 4.56 & $11 \mathrm{D}$ & 41.85 & 8.18 & $19 \mathrm{~J}$ & 72.25 & 5.59 & $27 \mathrm{~J}$ & 105.55 & 21.03 & $32 \mathrm{E}$ & 133.75 & 13.28 & $38 \mathrm{~A}$ & 155.10 & 26.11 \\
\hline $03 \mathrm{~K}$ & 11.35 & 6.96 & $11 \mathrm{C}$ & 42.35 & 11.00 & 19I & 72.75 & 4.82 & $27 \mathrm{I}$ & 106.05 & 12.76 & $32 \mathrm{D}$ & 134.25 & 7.82 & 38 & 155.55 & 21.95 \\
\hline $03 \mathrm{~J}$ & 11.85 & 7.49 & $11 \mathrm{~B}$ & 42.85 & 7.28 & $19 \mathrm{H}$ & 73.25 & 3.61 & $27 \mathrm{H}$ & 106.55 & 4.45 & $32 \mathrm{C}$ & 134.75 & 8.60 & $39 \mathrm{D}$ & 155.35 & 6.97 \\
\hline 03I & 12.35 & 7.59 & $11 \mathrm{~A}$ & 43.35 & 5.53 & $19 \mathrm{G}$ & 73.75 & 12.15 & $27 \mathrm{G}$ & 107.05 & 6.66 & $32 \mathrm{~B}$ & 135.25 & 13.33 & $39 \mathrm{C}$ & 155.85 & 7.80 \\
\hline $03 \mathrm{H}$ & 12.85 & 6.81 & $13 \mathrm{G}$ & 43.85 & 7.02 & $19 \mathrm{~F}$ & 74.25 & 14.11 & $27 \mathrm{~F}$ & 107.55 & 5.43 & $32 \mathrm{~A}$ & 135.80 & 17.27 & $39 \mathrm{~B}$ & 156.35 & 3.49 \\
\hline $03 \mathrm{G}$ & 13.35 & 7.18 & $13 \mathrm{~F}$ & 44.35 & 6.23 & $19 \mathrm{E}$ & 74.75 & 14.20 & $27 \mathrm{E}$ & 108.05 & 4.18 & $33 \mathrm{I}$ & 136.65 & 9.81 & 39A & 156.85 & 19.81 \\
\hline $03 \mathrm{~F}$ & 13.85 & 7.45 & $13 \mathrm{E}$ & 44.85 & 5.44 & 19D & 75.25 & 12.92 & $27 \mathrm{D}$ & 108.55 & 9.55 & $33 \mathrm{H}$ & 137.15 & 13.86 & $40 \mathrm{~B}$ & 157.75 & 4.88 \\
\hline $03 \mathrm{E}$ & 14.35 & 5.73 & $13 \mathrm{D}$ & 45.35 & 9.72 & $19 \mathrm{C}$ & 75.75 & 6.05 & $27 \mathrm{C}$ & 109.00 & 8.69 & $33 \mathrm{G}$ & 137.65 & 15.57 & $40 \mathrm{~A}$ & 158.25 & 4.65 \\
\hline 03D & 14.85 & 7.25 & $13 \mathrm{C}$ & 45.85 & 9.95 & 19B & 76.25 & 3.84 & $27 \mathrm{~B}$ & 109.55 & 7.29 & $33 \mathrm{~F}$ & 138.15 & 9.90 & 40 & 158.65 & 10.12 \\
\hline $03 \mathrm{C}$ & 15.35 & 7.53 & $13 \mathrm{~B}$ & 46.35 & 8.80 & 19A & 76.75 & 4.18 & $28 \mathrm{G}$ & 110.25 & 8.42 & $33 \mathrm{E}$ & 138.65 & 7.60 & $17 \mathrm{SK}$ & 159.75 & 8.39 \\
\hline 03B & 15.85 & 5.13 & $13 \mathrm{~A}$ & 46.85 & 12.89 & $20 \mathrm{H}$ & 78.25 & 3.32 & $28 \mathrm{~F}$ & 110.75 & 8.71 & $33 \mathrm{D}$ & 139.15 & 4.95 & 18SKA & 161 & 6.89 \\
\hline 03A & 16.35 & 5.99 & $14 \mathrm{~J}$ & 50.15 & 8.48 & $20 \mathrm{G}$ & 78.75 & 5.26 & $28 \mathrm{E}$ & 111.25 & 14.04 & $33 \mathrm{C}$ & 139.65 & 7.99 & 18SKB & 161.00 & 5.24 \\
\hline $04 \mathrm{H}$ & 17.15 & 5.07 & $14 \mathrm{I}$ & 50.65 & 8.00 & $20 \mathrm{~F}$ & 79.25 & 7.32 & $28 \mathrm{D}$ & 111.75 & 10.03 & $33 \mathrm{~B}$ & 140.15 & 15.49 & $18 \mathrm{SKC}$ & 166.50 & 2.95 \\
\hline $04 \mathrm{G}$ & 17.65 & 5.63 & $14 \mathrm{H}$ & 51.15 & 7.41 & $20 \mathrm{E}$ & 79.75 & 12.58 & $28 \mathrm{C}$ & 112.25 & 7.10 & $33 \mathrm{~A}$ & 140.65 & 11.49 & 19SKA & 166.50 & 1.35 \\
\hline $04 \mathrm{~F}$ & 18.15 & 7.24 & $14 \mathrm{G}$ & 51.65 & 9.89 & $20 \mathrm{D}$ & 80.25 & 16.67 & $28 \mathrm{~B}$ & 112.75 & 8.30 & $35 \mathrm{~K}$ & 141.05 & 11.47 & 19SKB & 176.00 & 1.24 \\
\hline 04E & 18.65 & 6.86 & $14 \mathrm{~F}$ & 52.15 & 11.01 & $21 \mathrm{E}$ & 82.00 & 2.58 & $28 \mathrm{~A}$ & 113.20 & 12.75 & $35 \mathrm{~J}$ & 141.35 & 13.24 & $41 \mathrm{~A}$ & 184.45 & 3.95 \\
\hline
\end{tabular}




\begin{tabular}{|c|c|c|c|c|c|c|c|c|c|c|c|c|c|c|c|c|c|}
\hline $\begin{array}{c}\text { Sample } \\
\text { ID }\end{array}$ & \begin{tabular}{|c|} 
Depth \\
(ft \\
bgs)
\end{tabular} & \begin{tabular}{|c|} 
Wt $\%$ \\
Moisture
\end{tabular} & $\begin{array}{c}\text { Sample } \\
\text { ID }\end{array}$ & \begin{tabular}{|c|} 
Depth \\
(ft \\
bgs)
\end{tabular} & \begin{tabular}{|c|} 
Wt $\%$ \\
Moisture
\end{tabular} & $\begin{array}{c}\text { Sample } \\
\text { ID }\end{array}$ & $\begin{array}{c}\text { Depth } \\
\text { (ft } \\
\text { bgs) }\end{array}$ & $\begin{array}{c}\text { Wt\% } \\
\text { Moisture }\end{array}$ & $\begin{array}{c}\text { Sample } \\
\text { ID }\end{array}$ & $\begin{array}{c}\text { Depth } \\
\text { (ft } \\
\text { bgs) }\end{array}$ & $\begin{array}{c}\text { Wt\% } \\
\text { Moisture }\end{array}$ & $\begin{array}{c}\text { Sample } \\
\text { ID }\end{array}$ & $\begin{array}{c}\text { Depth } \\
\text { (ft } \\
\text { bgs) }\end{array}$ & \begin{tabular}{c|} 
Wt $\%$ \\
Moisture
\end{tabular} & $\begin{array}{c}\text { Sample } \\
\text { ID }\end{array}$ & \begin{tabular}{|c|} 
Depth \\
$(\mathbf{f t}$ \\
bgs $)$
\end{tabular} & Moisture \\
\hline 04D & 19.15 & 7.26 & $14 \mathrm{E}$ & 52.65 & 6.80 & $21 \mathrm{D}$ & 83.00 & 3.55 & 29I & 113.85 & 8.91 & $35 \mathrm{I}$ & 141.85 & 24.48 & 19SKC & 185 & 0.88 \\
\hline $04 \mathrm{C}$ & 19.65 & 8.72 & $14 \mathrm{D}$ & 53.15 & 5.28 & $21 \mathrm{C}$ & 84.00 & 4.31 & $29 \mathrm{H}$ & 114.35 & 5.78 & $35 \mathrm{H}$ & 142.35 & 27.23 & 20SKA & 185 & 1.28 \\
\hline 04B & 20.15 & 7.94 & $15 \mathrm{~B}$ & 54.12 & 6.23 & $21 \mathrm{~B}$ & 85.00 & 2.94 & $29 \mathrm{G}$ & 114.85 & 4.22 & $35 \mathrm{G}$ & 142.85 & 27.15 & $20 \mathrm{SKB}$ & 189.5 & 1.14 \\
\hline $04 \mathrm{~A}$ & 20.65 & 7.25 & $15 \mathrm{~A}$ & 54.50 & 5.84 & $21 \mathrm{~A}$ & 86.00 & 2.89 & $29 \mathrm{~F}$ & 115.35 & 4.97 & $35 \mathrm{~F}$ & 143.35 & 26.00 & 42 & 194.55 & 5.87 \\
\hline $05 \mathrm{E}$ & 22.85 & 8.48 & $16 \mathrm{~B}$ & 56.95 & 6.59 & $22 \mathrm{E}$ & 87.10 & 7.72 & $29 \mathrm{E}$ & 115.85 & 5.51 & $35 \mathrm{E}$ & 143.85 & 25.08 & 43B & 204.50 & 6.16 \\
\hline $05 \mathrm{D}$ & 23.55 & 8.87 & $16 \mathrm{~A}$ & 57.45 & 6.32 & $22 \mathrm{D}$ & 88.10 & 16.49 & $29 \mathrm{D}$ & 116.35 & 5.60 & $35 \mathrm{D}$ & 144.35 & 16.57 & $43 \mathrm{~A}$ & 205.15 & 2.70 \\
\hline $05 \mathrm{C}$ & 24.65 & 7.77 & $17 \mathrm{~J}$ & 61.75 & 5.33 & $22 \mathrm{C}$ & 89.10 & 5.65 & $29 \mathrm{C}$ & 116.85 & 9.08 & $35 \mathrm{C}$ & 144.85 & 16.25 & & & \\
\hline 05B & 25.75 & 7.42 & $17 \mathrm{I}$ & 62.25 & 5.70 & $22 \mathrm{~B}$ & 91.10 & 8.12 & $29 A+B$ & 117.85 & 9.87 & $35 \mathrm{~B}$ & 145.35 & 11.50 & & & \\
\hline $05 \mathrm{~A}$ & 26.85 & 8.87 & $17 \mathrm{H}$ & 62.75 & 5.18 & $22 \mathrm{~A}$ & 91.10 & 5.27 & $30 \mathrm{G}$ & 123.85 & 7.86 & $35 \mathrm{~A}$ & 145.85 & 25.75 & & & \\
\hline $08 \mathrm{C}$ & 27.55 & 8.15 & $17 \mathrm{G}$ & 63.25 & 8.99 & $24 \mathrm{C}$ & 93.00 & 8.01 & $30 \mathrm{~F}$ & 124.35 & 10.12 & $36 \mathrm{~F}$ & 146.45 & 15.67 & & & \\
\hline 08B & 28.65 & 9.51 & $17 \mathrm{~F}$ & 63.75 & 11.77 & $24 \mathrm{~B}$ & 94.00 & 6.42 & $30 \mathrm{E}$ & 124.85 & 10.07 & $36 \mathrm{E}$ & 146.95 & 16.91 & & & \\
\hline 08A & 29.75 & 9.13 & $17 \mathrm{E}$ & 64.25 & 21.84 & $24 \mathrm{~A}$ & 95.00 & 9.25 & $30 \mathrm{D}$ & 125.35 & 9.78 & $36 \mathrm{D}$ & 147.45 & 9.36 & & & \\
\hline 09I & 30.95 & 8.46 & $17 \mathrm{D}$ & 64.75 & 20.18 & $25 \mathrm{E}$ & 96.06 & 4.46 & $30 \mathrm{C}$ & 125.85 & 13.18 & $36 \mathrm{C}$ & 147.95 & 8.52 & & & \\
\hline $09 \mathrm{H}$ & 31.45 & 9.69 & $17 \mathrm{C}$ & 65.25 & 11.13 & $25 \mathrm{D}$ & 97.06 & 4.47 & $30 \mathrm{~B}$ & 126.35 & 16.57 & $36 \mathrm{~B}$ & 148.45 & 22.95 & & & \\
\hline 09G & 31.95 & 8.23 & $17 \mathrm{~B}$ & 65.75 & 10.30 & $25 \mathrm{C}$ & 98.06 & 5.21 & $30 \mathrm{~A}$ & 126.85 & 13.86 & $36 \mathrm{~A}$ & 148.95 & 16.89 & & & \\
\hline 09F & 32.45 & 8.00 & $17 \mathrm{~A}$ & 66.25 & 9.34 & $25 \mathrm{~B}$ & 99.06 & 5.12 & $31 \mathrm{~J}$ & 127.80 & 12.27 & $37 \mathrm{E}$ & 149.15 & 13.30 & & & \\
\hline 09E & 32.95 & 10.16 & $18 \mathrm{~K}$ & 66.45 & 13.04 & $25 \mathrm{~A}$ & 100.06 & 11.54 & $31 \mathrm{I}$ & 128.15 & 7.26 & $37 \mathrm{D}$ & 149.65 & 12.95 & & & \\
\hline 09D & 33.45 & 9.18 & $18 \mathrm{~J}$ & 66.95 & 14.17 & $26 \mathrm{~J}$ & 100.45 & 12.18 & $31 \mathrm{H}$ & 128.65 & 11.59 & $37 \mathrm{C}$ & 150.15 & 15.33 & & & \\
\hline 09C & 33.95 & 8.11 & $18 \mathrm{I}$ & 67.45 & 17.48 & $26 \mathrm{I}$ & 100.95 & 17.79 & $31 \mathrm{G}$ & 129.15 & 15.12 & $37 \mathrm{~B}$ & 150.65 & 17.24 & & & \\
\hline 09B & 34.45 & 8.73 & $18 \mathrm{H}$ & 67.95 & 16.46 & $26 \mathrm{H}$ & 101.45 & 7.98 & $31 \mathrm{~F}$ & 129.65 & 16.32 & $37 \mathrm{~A}$ & 151.15 & 13.73 & & & \\
\hline 09A & 34.95 & 8.72 & $18 \mathrm{G}$ & 68.45 & 5.16 & $26 \mathrm{G}$ & 101.95 & 9.72 & $31 \mathrm{E}$ & 130.15 & 15.09 & $38 \mathrm{H}$ & 151.75 & 18.93 & & & \\
\hline $10 \mathrm{D}$ & 35.85 & 8.96 & $18 \mathrm{~F}$ & 68.95 & 9.88 & $26 \mathrm{~F}$ & 102.45 & 11.96 & $31 \mathrm{D}$ & 130.65 & 12.30 & $38 \mathrm{G}$ & 152.10 & 21.31 & & & \\
\hline
\end{tabular}

bgs = below ground surface 


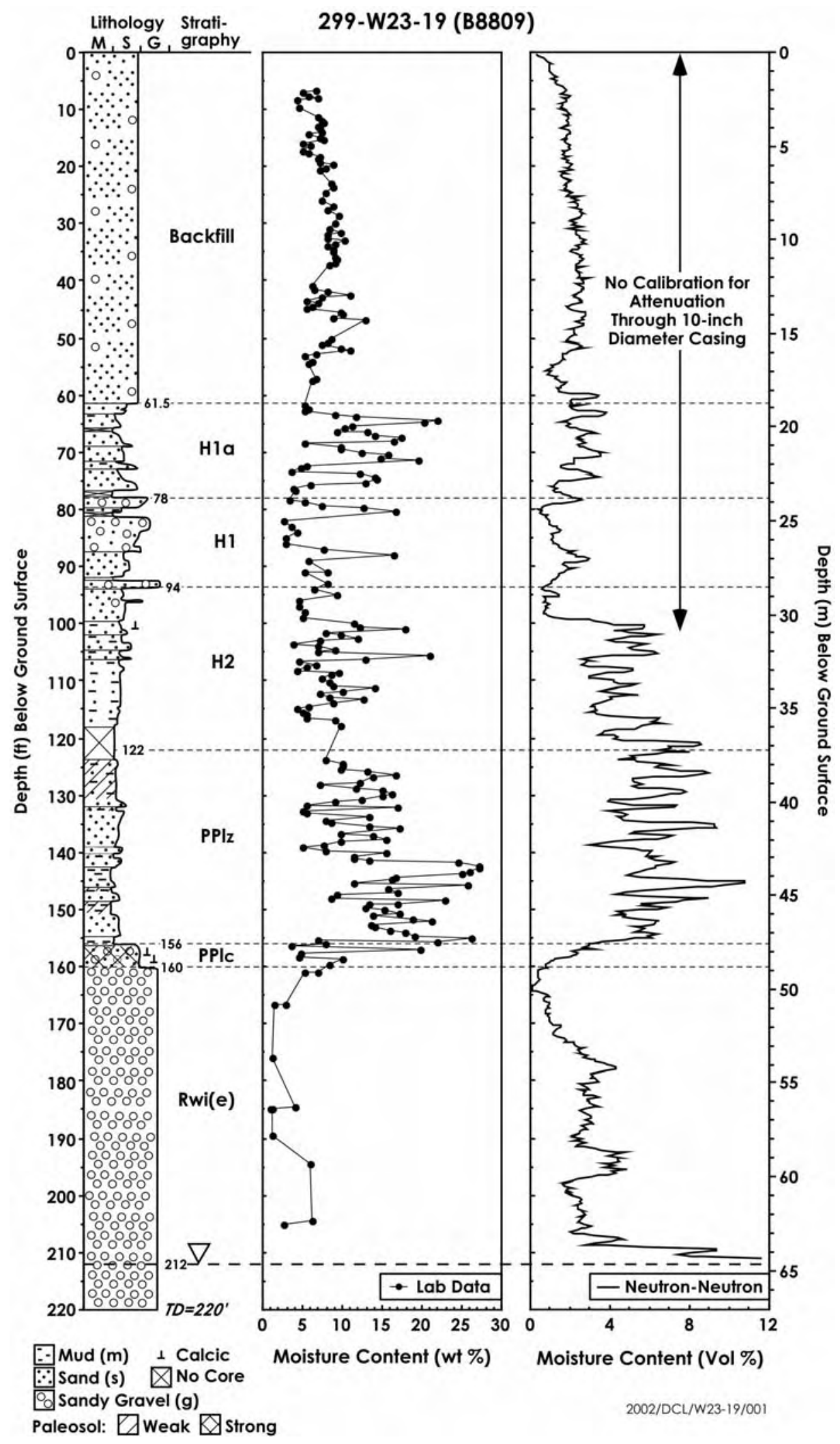

Figure 4.1 Moisture Content in Vadose Zone Sediment Profile at Borehole 299-W23-19 


\subsection{1:1 Sediment-to-Water Extracts}

This section gives the results of analysis of 1:1 sediment-to-water extracts from sediment samples from borehole 299-W23-19.

\subsection{1 pH and Electrical Conductivity}

Table 4.2 gives the results of $\mathrm{pH}$ and electrical conductivity measurements of water extracts. Figure 4.2 shows the same information versus depth in the borehole. The porewater electrical conductivity column in Table 4.2 and Figure 4.2 gives the conductivity of 1:1 extract corrected for dilution with deionized water (calculated by multiplying the water extract electrical conductivity by the dilution factor). The $\mathrm{pH}$ values in the table and figure are given as measured in the uncorrected extract.

The $\mathrm{pH}$ of uncontaminated sediment in the vadose zone is generally between 7 and 8 (Serne et al. 2002a). The data in Table 4.2 and Figure 4.2 show elevated pH between about 22.8 meters ( 75 feet) and 31.7 meters (104 feet) bgs in the Hanford formation H1a, H1 and upper part of $\mathrm{H} 2$ units. There may be two maxima with a minimum at about the $\mathrm{H} 1-\mathrm{H} 2$ boundary but the $\mathrm{pH}$ is quite variable throughout the profile. Nevertheless, the elevated $\mathrm{pH}$ suggests interactions between the sediment and caustic waste.

The porewater electrical conductivity profile in Figure 4.2 shows a broad, elevated gentle increase beginning at about 24.4 meters ( 80 feet bgs) and continuing through the Hanford formation $\mathrm{H} 1$ and $\mathrm{H} 2$ units. Electrical conductivity is extremely high in the lower part of the Hanford formation $\mathrm{H} 2$ unit and especially in the upper Plio-Pleistocene unit where electrical conductivity reaches $40,058 \mu \mathrm{S} / \mathrm{cm}$. Typical values for uncontaminated sediment are less than 10,000 $\mu \mathrm{s} / \mathrm{em}$ and usually less than 6,000 to 7,000 $\mu \mathrm{S} / \mathrm{cm}$. Based on the $\mathrm{pH}$ and electrical conductivity data, it appears that the leaked fluid traveled through the Hanford formation selectively reacting with the sediment (as indicated by increased $\mathrm{pH}$ ) and migrated into the upper Plio-Pleistocene unit. Electrical conductivity is within the normal range for Hanford Site sediment in the lower Plio-Pleistocene unit and the Ringold Formation.

The $\mathrm{pH}$ and electrical conductivity of the six samples of porewater obtained by ultracentrifuge (labeled with the suffix "UFA" in Tables 4.2 and 4.3) are compared with the 1:1 water extracts from the same depth in Table 4.3. For four samples, water extracts were obtained from the same sediment after the ultracentrifuge sample was obtained. These four samples are labeled with the suffix "-aft" in Tables 4.2 and 4.3. The other two 1:1 water extracts in Table 4.3 were performed on different aliquots of sediment than what was used for ultracentrifugation. The $\mathrm{pH}$ and electrical conductivity of the water extract samples were measured at least 6 months before that of the ultracentrifuge samples (Table 4.3). The pH and electrical conductivity for samples 19D-ufa and 20E-ufa (Table 4.3) were measured about 2 months after the samples were obtained. The resulting $\mathrm{pH}$ values were at least $1 \mathrm{pH}$ unit lower than the originally measured values from the water extract sample. This led to the remeasurement of $\mathrm{pH}$ and electrical conductivity on the two comparable 1:1 water extracts about 9 months after the original measurements. The after storage $\mathrm{pH}$ values for these two extracts were significantly lower than the original values. This might be caused by reaction with carbon dioxide from the atmosphere if the sample was undersaturated with carbon dioxide when obtained. 
Table 4.2 Water Extract pH and Electrical Conductivity Values

\begin{tabular}{|c|c|c|c|c|c|c|c|c|c|c|c|}
\hline Sample ID & $\begin{array}{c}\text { Depth } \\
\left(\mathbf{f t} \text { bgs }^{\mathrm{a}}\right)\end{array}$ & Dil. Fac. & $\begin{array}{l}1: 1 \\
\text { pH }\end{array}$ & $\begin{array}{c}1: 1 \\
\mathrm{EC} \\
(\mu \mathrm{S} / \mathrm{cm})\end{array}$ & \begin{tabular}{|c|} 
Dilution \\
Corrected \\
EC \\
$(\mu \mathrm{S} / \mathrm{cm})$ \\
\end{tabular} & Sample ID & $\begin{array}{r}\text { Depth } \\
\left(\mathbf{f t} \text { bgs }^{\mathbf{a}}\right)\end{array}$ & Dil. Fac. & $\begin{array}{l}1: 1 \\
\text { pH }\end{array}$ & $\begin{array}{c}1: 1 \\
\mathrm{EC} \\
(\mu \mathrm{S} / \mathrm{cm})\end{array}$ & \begin{tabular}{|c|} 
Dilution \\
Corrected \\
EC \\
$(\mu \mathrm{S} / \mathrm{cm})$ \\
\end{tabular} \\
\hline 09E & 32.95 & 9.85 & 7.94 & 166 & 1635 & $31 \mathrm{~A}$ & 132.15 & 6.36 & 6.95 & 5198 & 33066 \\
\hline $15 \mathrm{~A}$ & 54.50 & 17.13 & 7.25 & 233 & 3992 & $32 \mathrm{D}$ & 134.25 & 11.80 & 6.96 & 1658 & 19561 \\
\hline $16 \mathrm{~A}$ & 57.45 & 15.82 & 7.27 & 226 & 3576 & $32 \mathrm{~A}$ & 135.80 & 5.79 & 6.8 & 4973 & 28781 \\
\hline $17 \mathrm{~J}$ & 61.75 & 18.84 & 7.57 & 153 & 2883 & $33 \mathrm{G}$ & 137.65 & 6.43 & 6.82 & 4860 & 31270 \\
\hline $17 \mathrm{G}$ & 63.25 & 11.13 & 7.52 & 186 & 2071 & 33B & 140.15 & 6.45 & 6.81 & 4840 & 31238 \\
\hline $17 \mathrm{~F}$ & 63.75 & 8.53 & 7.41 & 217 & 1851 & $35 \mathrm{~K}$-aft & 141.05 & 8.72 & 6.9 & 4593 & 40058 \\
\hline 17F-dup & 63.75 & 8.56 & 7.1 & 211 & 1807 & 35IH-aft & 141.85 & 6.30 & 6.88 & 4265 & 26852 \\
\hline 17DE-aft & 64.75 & 10.70 & 7.48 & 206 & 2204 & 35F-aft & 143.35 & 7.18 & 6.93 & 3264 & 23439 \\
\hline $17 \mathrm{C}$ & 65.25 & 9.03 & 7.32 & 160 & 1445 & $35 \mathrm{C}$ & 144.85 & 6.15 & 6.95 & 2830 & 17409 \\
\hline $18 \mathrm{I}$ & 67.45 & 5.74 & 7.44 & 243 & 1394 & $35 \mathrm{~A}$ & 145.85 & 3.89 & 6.86 & 4315 & 16781 \\
\hline $18 \mathrm{E}$ & 69.45 & 10.19 & 7.62 & 226 & 2302 & $36 \mathrm{D}$ & 147.45 & 10.20 & 6.98 & 1593 & 16254 \\
\hline $18 \mathrm{CB}$ & 70.45 & 6.56 & 7.91 & 431 & 2826 & $36 \mathrm{~B}$ & 148.45 & 4.35 & 7.02 & 2655 & 11562 \\
\hline 18CB-dup & 70.95 & 6.56 & 7.46 & 413 & 2709 & $37 \mathrm{D}$ & 149.65 & 7.72 & 6.96 & 3792 & 29278 \\
\hline $19 \mathrm{H}$ & 73.25 & 27.70 & 8.61 & 603 & 16701 & $38 \mathrm{G}$ & 152.10 & 4.32 & 7.21 & 979 & 4232 \\
\hline $19 \mathrm{G}$ & 73.75 & 7.49 & 8.33 & 1207 & 9038 & $38 \mathrm{E}$ & 153.10 & 7.11 & 7.37 & 624 & 4439 \\
\hline $19 \mathrm{~F}$ & 74.25 & 7.09 & 7.71 & 1248 & 8847 & 38E-dup & 153.10 & 7.11 & 7.43 & 604 & 4297 \\
\hline 19D & 75.25 & 9.61 & 9.35 & 1039 & 9980 & $38 \mathrm{~A}-\mathrm{aft}$ & 155.10 & 10.92 & 7.04 & 280 & 3057 \\
\hline $20 \mathrm{~F}$ & 79.25 & 9.47 & 9.12 & 1094 & 10364 & 39D & 155.35 & 9.49 & 7.39 & 768 & 7288 \\
\hline 20F-dup & 79.25 & 9.38 & 9.15 & 1025 & 9617 & $39 \mathrm{C}$ & 155.85 & 12.83 & 6.83 & 352 & 4516 \\
\hline $20 \mathrm{E}$ & 79.75 & 7.79 & 9.28 & 1014 & 7902 & 39C-dup & 155.85 & 12.82 & 6.97 & 342 & 4385 \\
\hline $20 \mathrm{D}$ & 80.25 & 6.00 & 8.5 & 1198 & 7184 & $40 \mathrm{~B}$ & 157.75 & 20.49 & 7.68 & 201 & 4119 \\
\hline $21 \mathrm{~A}$ & 86.00 & 13.58 & 9.72 & 762 & 10350 & 40 & 158.65 & 10.96 & 7.65 & 297 & 3256 \\
\hline $22 \mathrm{D}$ & 88.10 & 6.07 & 7.79 & 1328 & 8056 & $17 \mathrm{SK}$ & 159.75 & 11.92 & 7.7 & 281 & 3350 \\
\hline $24 \mathrm{~A}$ & 95.00 & 10.82 & 7.91 & 1023 & 11065 & 18SKA & 161 & 14.68 & 7.64 & 251 & 3684 \\
\hline $25 \mathrm{~B}$ & 99.06 & 14.17 & 9.08 & 693 & 9822 & $18 \mathrm{SKB}$ & 161.00 & 19.78 & 7.05 & 252 & 4984 \\
\hline 25B-dup & 99.06 & 15.85 & 8.74 & 595 & 9430 & $18 \mathrm{SKC}$ & 166.50 & 34.13 & 7.26 & 148 & 5051 \\
\hline $25 \mathrm{~A}$ & 100.06 & 11.40 & 7.83 & 1071 & 12212 & 19SKA & 166.50 & 74.25 & 7.24 & 124 & 9207 \\
\hline $26 \mathrm{E}$ & 102.95 & 13.75 & 8.93 & 639 & 8786 & 19SKB & 176.00 & 80.64 & 7.26 & 116 & 9354 \\
\hline $26 \mathrm{~A}$ & 104.95 & 12.21 & 9.31 & 592 & 7226 & $41 \mathrm{~A}$ & 184.45 & 25.37 & 7.9 & 85 & 2157 \\
\hline $27 \mathrm{G}$ & 107.05 & 15.02 & 7.84 & 944 & 14182 & 19SKC & 185 & 114.65 & 8.38 & 85 & 9745 \\
\hline $29 \mathrm{DC}$ & 116.35 & 13.67 & 7.33 & 1124 & 15363 & 20SKA & 185 & 78.06 & 7.62 & 93 & 7260 \\
\hline
\end{tabular}




\begin{tabular}{|c|c|c|c|c|c|c|c|c|c|c|c|}
\hline Sample ID & $\begin{array}{c}\text { Depth } \\
\left(\mathbf{f t} \text { bgs }^{\mathrm{a}}\right)\end{array}$ & Dil. Fac. & $\begin{array}{l}1: 1 \\
\text { pH }\end{array}$ & $\begin{array}{c}1: 1 \\
\mathrm{EC} \\
(\mu \mathrm{S} / \mathrm{cm})\end{array}$ & $\begin{array}{c}\text { Dilution } \\
\text { Corrected } \\
\text { EC } \\
(\mu \mathrm{S} / \mathrm{cm})\end{array}$ & Sample ID & $\begin{array}{c}\text { Depth } \\
\left(\mathbf{f t} \text { bgs }^{\mathrm{a}}\right)\end{array}$ & Dil. Fac. & $\begin{array}{l}\text { 1:1 } \\
\text { pH }\end{array}$ & $\begin{array}{c}1: 1 \\
\mathrm{EC} \\
(\mu \mathrm{S} / \mathrm{cm})\end{array}$ & \begin{tabular}{|c|} 
Dilution \\
Corrected \\
EC \\
$(\mu \mathrm{S} / \mathrm{cm})$ \\
\end{tabular} \\
\hline $30 \mathrm{GF}$ & 123.85 & 11.13 & 7.02 & 1922 & 21390 & 20SKB & 189.5 & 82.45 & 7.43 & 101 & 8327 \\
\hline $30 \mathrm{D}$ & 125.35 & 6.78 & 6.96 & 3902 & 26448 & 42 & 194.55 & 17.10 & 7.7 & 142 & 2428 \\
\hline 30B & 126.35 & 6.03 & 6.61 & 5730 & 34580 & $43 \mathrm{~B}$ & 204.50 & 16.25 & 7.52 & 273 & 4435 \\
\hline $31 \mathrm{G}$ & 129.15 & 6.62 & 6.71 & 6907 & 45724 & $43 \mathrm{~A}$ & 205.15 & 41.39 & 7.86 & 112 & 4636 \\
\hline \multicolumn{12}{|c|}{\begin{tabular}{|l} 
(a) multiply by 0.3048 to convert to meters \\
dup = duplicate \\
aft = extract sample analyzed after the ultracentrifuge sample \\
EC = electrical conductivity
\end{tabular}} \\
\hline
\end{tabular}

Comparison of the actual porewater, obtained with the ultracentrifuge, and the calculated porewater from the 1:1 water extract is not straightforward because of the different values of $\mathrm{pH}$ obtained at different times after sample collection. However, below the depth at which the water extracts suggest evidence of caustic reactions, the $\mathrm{pH}$ for the water extract and ultracentrifuge samples are comparable.

Comparison of the electrical conductivity measurements between the actual porewaters and the dilution-corrected values is very good for samples from the highest salt portion of the plume. However, within the leading and trailing edges of the salt plume, the actual porewaters are slightly less salty than the dilution-corrected porewaters. Generally, the electrical conductivity of dilution-corrected water is 30 to 50 times higher than that of the actual porewater below the main plume.

\subsubsection{Anions and Cations}

The anion composition of the 1:1 sediment-to-water extracts and the dilution-corrected porewaters is shown in Table 4.4 and Figure 4.3. The actual anion porewater compositions, as measured from samples collected with the ultracentrifuge, are shown in Table 4.5 and Figure 4.3. The most obvious feature shown by the anion trends on Figure 4.3 is the increase in nitrate concentration beginning just below the tank at about 19.8 meters (65 feet) and increasing to as much as 26,000 milligrams per liter in the upper Plio-Pleistocene. Increased nitrate over typical background values continues to near the base of the upper Plio-Pleistocene at 47.5 meters (156 feet bgs). Typical nitrate concentrations in uncontaminated Hanford formation and Plio-Pleistocene units are less than 300 to 400 milligrams per liter (Serne et al. 2002a). A small nitrite plume is present in the upper Plio-Pleistocene unit between 37.2 and 42.7 meters (122 and 140 feet bgs). Both nitrate and nitrite decrease with depth to concentrations near those found in uncontaminated boreholes (Serne et al. 2002a).

The sulfate concentration slowly increases from about 150 milligrams per liter at 19.8 meters ( 65 feet bgs) to a maximum of 935 milligrams per liter at 37.8 meters (124 feet bgs) just below the base of the Hanford formation $\mathrm{H} 2$ unit. The sulfate decreases to background concentrations in the upper PlioPleistocene unit. Sulfate within the Ringold Formation is similar to concentrations near the bottom of uncontaminated boreholes. Baseline values for sulfate throughout the sediment column at the Hanford Site is less than 500 milligrams per liter and usually less than 300 milligrams per liter. 
The chloride profile shown in Figure 4.3 is similar to that found in two uncontaminated boreholes (Serne et al. 2002a) throughout the Hanford formation. However, within the Plio-Pleistocene unit, the chloride concentrations in the dilution-corrected porewater appear slightly elevated at up to 215 milligrams per liter. Chloride concentrations of extract of uncontaminated upper Plio-Pleistocene sediment are generally less than 120 milligrams per liter. Below the upper Plio-Pleistocene unit, chloride concentrations are similar to those found in uncontaminated sediment. Similarly, fluoride concentrations of the dilution-corrected porewaters are similar to the concentrations found in uncontaminated sediment (Serne et al. 2002a). There is definitely more leachable fluoride in the Ringold Formation than in the overlying sediment except for one sample in the Hanford formation Hla Unit.

Table 4.3 Comparison of Actual Porewater pH and Electrical Conductivity with Dilution-corrected Water Extract Values

\begin{tabular}{|c|c|c|c|c|c|c|}
\hline Sample ID & $\begin{array}{c}\text { Depth } \\
(\text { ft bgs })^{a}\end{array}$ & Dil. Fac. & pH & $\begin{array}{c}\mathrm{pH} \\
\text { after storage }\end{array}$ & $\begin{array}{c}1: 1 \\
\text { Extract EC } \\
(\mu \mathrm{S} / \mathrm{cm})\end{array}$ & \begin{tabular}{|c} 
Ultracentrifuge \\
EC \\
$(\mu \mathrm{S} / \mathrm{cm})$
\end{tabular} \\
\hline 17DE-aft & 64.75 & 10.70 & 7.48 & & 206 & 2204 \\
\hline 17DE-ufa & & & 7.04 & & & 829 \\
\hline $19 \mathrm{D}$ & 75.25 & 9.61 & 9.35 & 8.3 & 1039 & 9980 \\
\hline 19D-ufa & & & & 8.15 & & 6487 \\
\hline $20 \mathrm{E}$ & 79.75 & 7.79 & 9.28 & 8.6 & 1014 & 7902 \\
\hline 20E-ufa & & & & 8.13 & & 5884 \\
\hline 35IH-aft & 141.85 & 6.30 & 6.88 & & 4265 & 26852 \\
\hline 35IH-ufa & & & 6.47 & & & 25710 \\
\hline 35F-aft & 143.35 & 7.18 & 6.93 & & 3264 & 23439 \\
\hline $35 \mathrm{~F}-\mathrm{ufa}$ & & & 6.63 & & & 23390 \\
\hline 38A-aft & 155.10 & 10.92 & 7.04 & & 280 & 3057 \\
\hline 38A-ufa & & & 7.00 & & & 2032 \\
\hline \multicolumn{7}{|c|}{$\begin{array}{l}\text { (a) multiply by } 0.3048 \text { to convert to meters } \\
\text { aft = extract sample analyzed after the ultracentrifuge sample } \\
\text { EC = electrical conductivity } \\
\text { ufa = obtained by ultracentrifuge }\end{array}$} \\
\hline
\end{tabular}

The anion concentrations, which are generally good indicators of subsurface, mobile contaminant distribution, show that tank fluids have contacted the sediment in borehole 299-W23-19 between about 19.8 and 47.5 meters ( 65 and 156 feet bgs). The main portion of the plume resides in the fine-grained, upper Plio-Pliestocene unit where there is significant elevated nitrate and somewhat higher chloride than in typical Hanford Site sediment. There is no evidence in the anion data that contamination has penetrated into or through the lower Plio-Pleistocene caliche unit and into the underlying Ringold Formation. This is somewhat contradictory to the discussion in Section 4.2.10 below on soil suction measurements that suggest that the vadose zone sediment at borehole 299-W23-19 is draining all the way 
to the water table. However, only one soil suction measurement was obtained below the lower PlioPliestocene unit.

Sulfate, which is more reactive than the other anions, appears to be elevated in the Hanford formation $\mathrm{H} 2$ unit between 30.5 and 38.1 meters (100 and 125 feet bgs). The relatively high sulfate concentration in this part of the borehole 299-W23-19 may be from leaked tank fluids or may be partially a result of sulfate added to the porewater from anion exchange of nitrate for sulfate shallower in the borehole.

Table 4.5 shows data comparing the dilution-corrected porewater composition with the anion composition of actual porewaters obtained by ultracentrifugation. The comparison is hampered by several things. In only two cases (19D/19D-ufa and 20E/20E-ufa) did the water extract and ultracentrifuge sediment come from the same borehole sleeve. Also, for two other cases, the 1:1 water extract was not done correctly such that the dilution factor, and, thus, the dilution-corrected concentrations, are incorrect. However, the overall anion profiles in Figure 4.3 give a good indication of the depth to which leaked fluids have penetrated.

The cation concentrations in porewater from the vadose zone sediment at borehole 299-W23-19 are shown in Table 4.6. The depth distribution of several of the major cations is shown in Figure 4.4. The major alkali and alkaline earth cations barium, calcium, magnesium, potassium, and strontium show remarkable similarity in their depth distribution. Each of these cations shows a large increase in the amount that is water-extractable between $\sim 35.3$ and 45.7 meters (116 and 150 feet bgs). The next shallower sample analyzed above 35.3 meters (116 feet) is from 32.6 meters (107 feet bgs) so that a more precise depth at which the increase begins cannot be determined.

Figure 4.4 and Table 4.7 give data comparing the actual porewater concentration, obtained by centrifugation, and dilution-corrected porewater concentrations. In general, results from both analyses are similar.

All five cations in Figure 4.4 show a progressive decrease in water-extractable amounts below about 42.7 meters (140 feet) bgs except for sample 37D. Coincidently, the drillers' log notes an equipment malfunction during collection of the next deepest split-spoon (sample 38G). The split-spoon sampler remained in the borehole for 73 hours before it could be removed and samples processed for delivery to the laboratory. It is not known whether there is any relationship between the sharp decrease in concentration of water-extractable cations and the delay in removing the split-spoon sampler. However, the next two deeper samples between $38 \mathrm{G}$ and the bottom of the upper Plio-Pleistocene unit are very similar suggesting that there is no relationship between concentration and equipment malfunction.

The sodium concentration in samples from borehole 299-W23-19 is elevated between 22.2 and 38.4 meters (73 and 126 feet bgs), just below the contact between Hanford formation H2 unit and the upper Plio-Pleistocene unit. The bottom of the zone with elevated sodium is not sharp, and higher than natural sodium concentrations (less than 600 milligrams per liter, see Serne et al. 2002a) can be followed to a depth of 43.6 meters (143 feet bgs) within the upper Plio-Pleistocene unit.

The actual porewater from the Hanford formation has lower barium concentrations than do the dilution-corrected water extractsexcept in the zone of high contamination in the upper Plio-Pliestocene. The actual porewater from the Hanford formation sediment has slightly higher calcium concentrations 


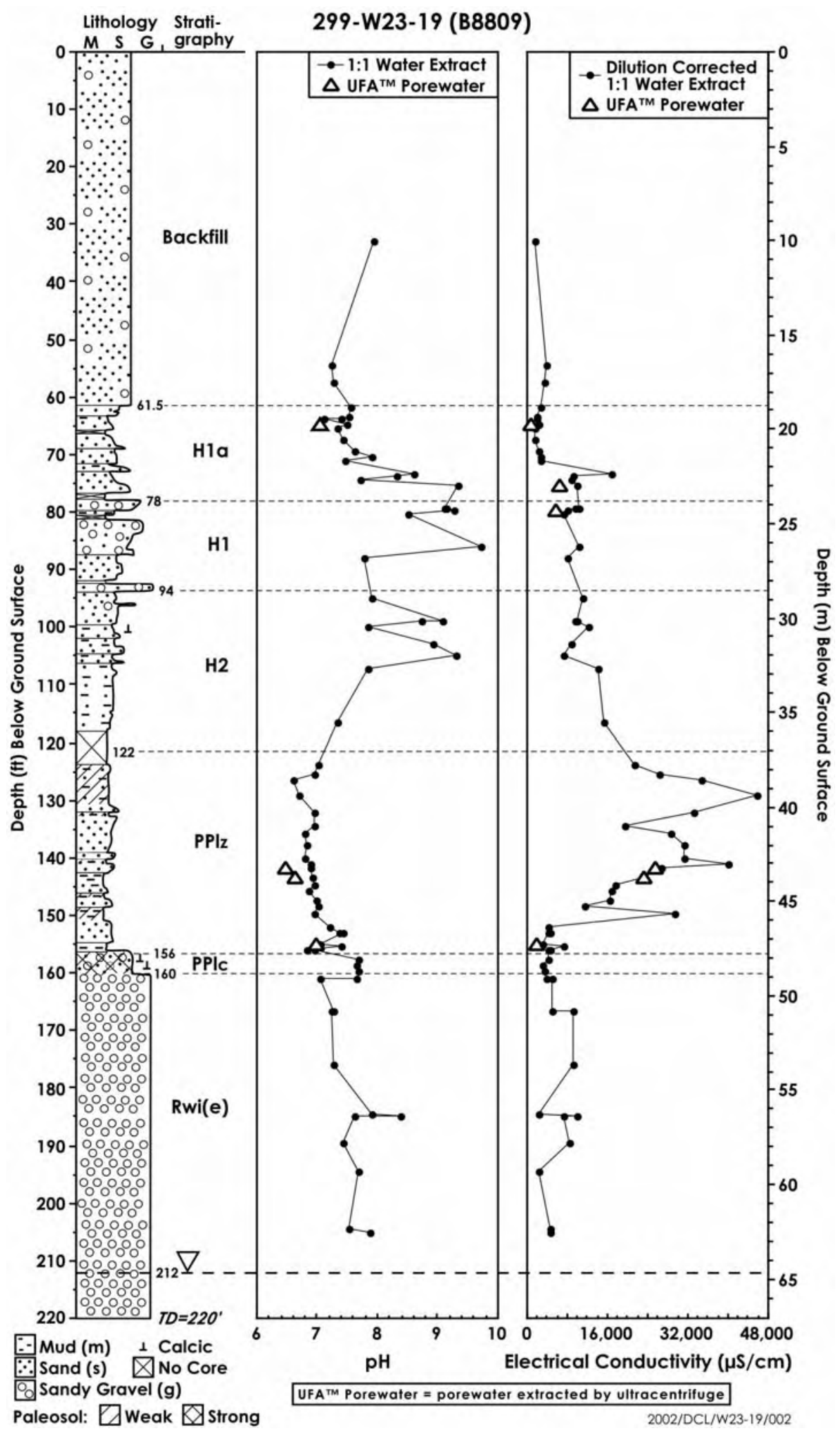

Figure $4.2 \mathrm{pH}$ and Electrical Conductivity for Dilution-Corrected and Actual Porewaters from Borehole 299-W23-19 

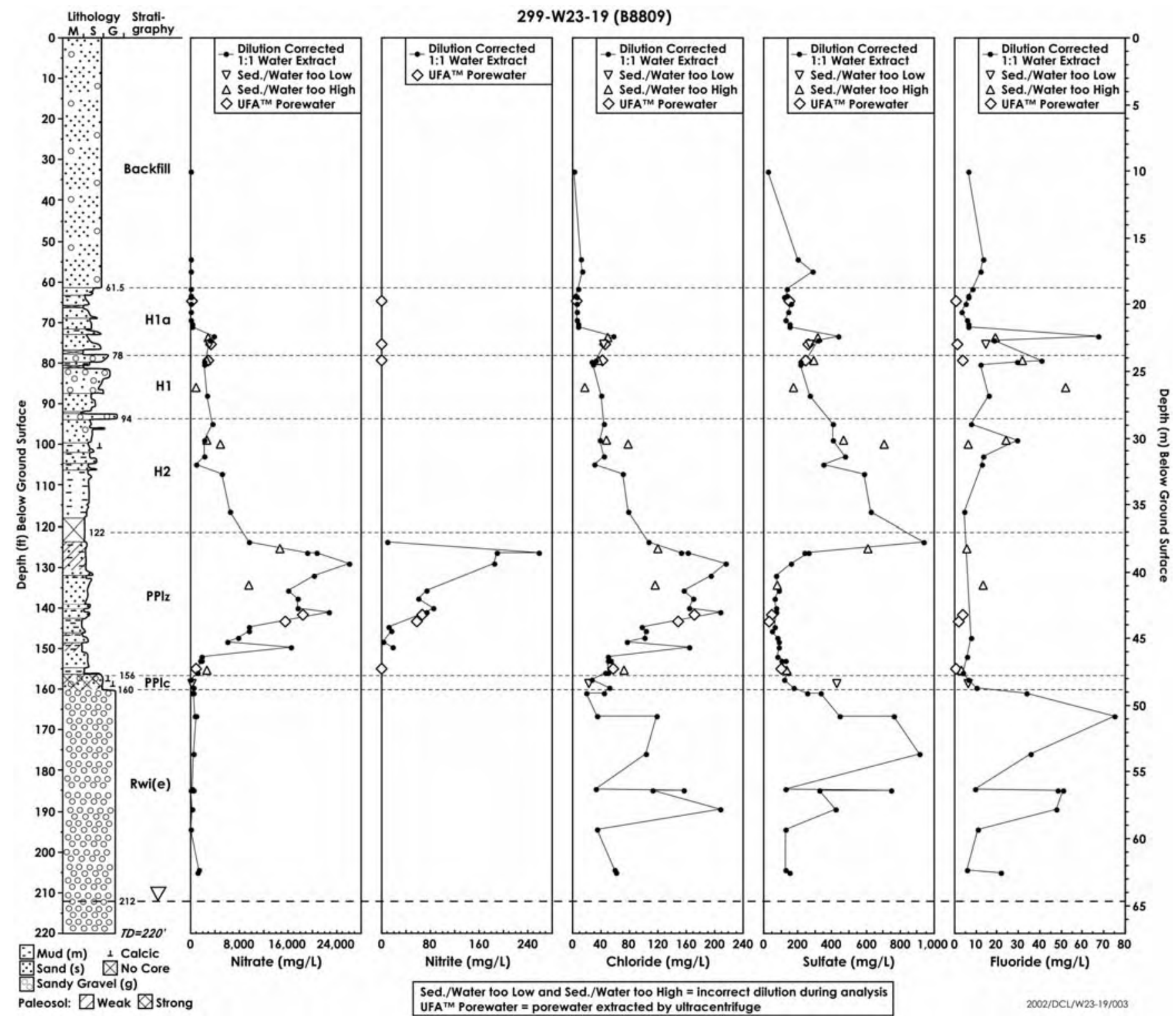

Figure 4.3 Dilution-Corrected (from sediment:water extracts) and Actual (from ultracentrifuge) Porewater Anion Concentrations for Borehole 299-W23-19 Sediment. 
Table 4.4 Anion Concentrations of Water Extracts from Borehole 299-W23-19 Sediment Samples

\begin{tabular}{|c|c|c|c|c|c|c|c|c|c|c|c|c|c|c|c|c|c|}
\hline \multirow[b]{2}{*}{$\begin{array}{c}\text { Sample } \\
\text { ID }\end{array}$} & \multirow{2}{*}{$\begin{array}{l}\text { Depth } \\
\text { (ft bgs) }\end{array}$} & \multirow[b]{2}{*}{ Dil. Fac. } & \multirow{2}{*}{$\begin{array}{c}\text { water:soil } \\
\text { Ratio }\end{array}$} & \multicolumn{7}{|c|}{ 1:1 Extracts Concentrations ( milligrams per liter) } & \multicolumn{7}{|c|}{ Dilution-Corrected Porewater Concentrations (milligrams per liter) } \\
\hline & & & & Nitrate & Fluoride & Nitrite & Chloride & Sulfate & Phosphate & Alk ${ }^{*}$ & Nitrate & Nitrite & Chloride & Sulfate & Phosphate & Fluoride & Alk ${ }^{*}$ \\
\hline 09E & 32.95 & 9.85 & 1.00 & 1.00 & 0.64 & $<0.04$ & 0.16 & 2.34 & 0.14 & 124 & 10 & $<0.39$ & 1.6 & 23 & 1.4 & 6.3 & 1219 \\
\hline $15 \mathrm{~A}$ & 54.50 & 17.13 & 1.00 & 0.76 & 0.77 & $<0.04$ & 0.71 & 11.66 & $<0.1$ & 96 & 13 & $<0.69$ & 12.2 & 200 & 1.7 & 13.2 & 1647 \\
\hline $16 \mathrm{~A}$ & 57.45 & 15.82 & 1.00 & 3.10 & 0.78 & $<0.04$ & 0.91 & 18.33 & 0.10 & 305 & 49 & $<0.63$ & 14.4 & 290 & 1.6 & 12.3 & 4830 \\
\hline $17 \mathrm{~J}$ & 61.75 & 18.84 & 1.00 & 1.35 & 0.45 & $<0.04$ & 0.37 & 7.14 & $<0.1$ & 58 & 25 & $<0.75$ & 7.0 & 135 & 1.9 & 8.5 & 1101 \\
\hline $17 \mathrm{G}$ & 63.25 & 11.13 & 1.00 & 5.25 & 0.60 & $<0.04$ & 0.39 & 11.86 & 0.12 & 83 & 58 & $<0.45$ & 4.3 & 132 & 1.3 & 6.7 & 921 \\
\hline $17 \mathrm{~F}$ & 63.75 & 8.53 & 1.00 & 8.83 & 0.72 & $<0.04$ & 0.60 & 15.77 & 0.19 & 78 & 75 & $<0.34$ & 5.1 & 135 & 1.6 & 6.1 & 664 \\
\hline 17FDUP & 63.75 & 8.56 & 1.01 & 8.3 & 0.73 & $<0.08$ & 0.5 & 13.67 & 0.18 & & 71 & $<0.69$ & 4.3 & 117 & 1.5 & 6.3 & \\
\hline $17 \mathrm{C}$ & 65.25 & 9.03 & 1.00 & 12.43 & 0.55 & $<0.04$ & 0.62 & 17.31 & 0.24 & 65 & 112 & $<0.36$ & 5.6 & 156 & 2.2 & 5.0 & 586 \\
\hline $18 \mathrm{I}$ & 67.45 & 5.74 & 1.00 & 19.93 & 0.57 & $<0.04$ & 0.91 & 25.14 & 0.44 & 78 & 114 & $<0.23$ & 5.2 & 144 & 2.5 & 3.3 & 447 \\
\hline $18 \mathrm{E}$ & 69.45 & 10.19 & 1.00 & 10.26 & 0.54 & $<0.04$ & 0.66 & 12.66 & 0.30 & 91 & 105 & $<0.41$ & 6.7 & 129 & 3.1 & 5.5 & 928 \\
\hline $18 \mathrm{C}$ & 70.45 & 6.56 & 1.00 & 28.02 & 1.02 & $<0.04$ & 1.20 & 22.91 & 1.24 & 160 & 184 & $<0.26$ & 7.9 & 150 & 8.1 & 6.7 & 1047 \\
\hline $18 \mathrm{~B}$ & 70.95 & 6.56 & 1.00 & 27.69 & 1.02 & $<0.08$ & 1.28 & 23.02 & 1.76 & & 182 & $<0.52$ & 8.4 & 151 & 11.5 & 6.7 & \\
\hline $19 \mathrm{H}$ & 73.25 & 27.70 & 1.00 & 136.83 & 2.43 & $<0.1$ & 2.05 & 15.71 & 0.62 & & 3790 & $<2.8$ & 56.8 & 435 & 17.2 & 67.3 & \\
\hline $19 \mathrm{G}$ & 73.75 & 7.49 & 0.86 & 400.86 & 2.54 & $<0.1$ & 6.73 & 42.45 & 0.74 & & 3002 & $<0.7$ & 50.4 & 318 & 5.5 & 19.0 & \\
\hline $19 \mathrm{~F}$ & 74.25 & 7.09 & 1.00 & 431.09 & 2.64 & $<0.04$ & 6.69 & 44.49 & $<1$ & 131 & 3056 & $<0.28$ & 47.4 & 315 & $<7.1$ & 18.7 & 931 \\
\hline $19 \mathrm{D}$ & 75.25 & 9.61 & 1.18 & 321.44 & 1.52 & $<0.1$ & 4.6 & 28.31 & 0.89 & & 3088 & $<1.0$ & 44.2 & 272 & 8.5 & 14.6 & \\
\hline $20 \mathrm{~F}$ & 79.25 & 9.47 & 0.76 & 292.01 & 3.35 & $<0.1$ & 3.87 & 31.17 & 1.19 & & 2766 & $<0.9$ & 36.7 & 295 & 11.3 & 31.7 & \\
\hline 20FDUP & 79.25 & 9.38 & 1.04 & 250.53 & 4.36 & $<0.1$ & 3.43 & 25.87 & 1.3 & & 2351 & $<0.9$ & 32.2 & 243 & 12.2 & 40.9 & \\
\hline $20 \mathrm{E}$ & 79.75 & 7.79 & 1.00 & 275.95 & 3.78 & $<0.1$ & 3.43 & 27.1 & 1.37 & & 2150 & $<0.8$ & 26.7 & 211 & 10.7 & 29.5 & \\
\hline $20 \mathrm{D}$ & 80.25 & 6.00 & 1.00 & 358.65 & 2.01 & $<0.04$ & 4.63 & 35.21 & 3.16 & 175 & 2151 & $<0.24$ & 27.8 & 211 & 18.9 & 12.1 & 1051 \\
\hline $21 \mathrm{~A}$ & 86.00 & 13.58 & 0.61 & 73.51 & 3.83 & $<0.1$ & 1.21 & 12.68 & 0.67 & & 998 & $<1.4$ & 16.4 & 172 & 9.1 & 52.0 & \\
\hline $22 \mathrm{D}$ & 88.10 & 6.07 & 1.00 & 446 & 2.62 & $<0.04$ & 6.46 & 44.80 & 1.61 & 182 & 2716 & $<0.24$ & 39.5 & 274 & 10.7 & 15.9 & 1105 \\
\hline $24 \mathrm{~A}$ & 95.00 & 10.82 & 1.00 & 340.37 & 0.72 & $<0.04$ & 4.11 & 37.24 & 1.45 & 215 & 3682 & $<0.43$ & 44.5 & 403 & 15.7 & 7.8 & 2320 \\
\hline
\end{tabular}




\begin{tabular}{|c|c|c|c|c|c|c|c|c|c|c|c|c|c|c|c|c|c|}
\hline \multirow[b]{2}{*}{$\begin{array}{c}\text { Sample } \\
\text { ID }\end{array}$} & \multirow{2}{*}{$\begin{array}{l}\text { Depth } \\
\text { (ft bgs) }\end{array}$} & \multirow[b]{2}{*}{ Dil. Fac. } & \multirow{2}{*}{$\begin{array}{c}\text { water:soil } \\
\text { Ratio } \\
\end{array}$} & \multicolumn{7}{|c|}{ 1:1 Extracts Concentrations ( milligrams per liter) } & \multicolumn{7}{|c|}{ Dilution-Corrected Porewater Concentrations (milligrams per liter) } \\
\hline & & & & Nitrate & Fluoride & Nitrite & Chloride & Sulfate & Phosphate & Alk* & Nitrate & Nitrite & Chloride & Sulfate & Phosphate & Fluoride & Alk* \\
\hline $25 \mathrm{~B}$ & 99.06 & 14.17 & 0.81 & 183.64 & 1.71 & $<0.1$ & 3.42 & 33.28 & 0.83 & & 2603 & $<1.4$ & 48.5 & 472 & 11.8 & 24.2 & \\
\hline 25Bdup & 99.06 & 15.85 & 0.97 & 141.66 & 1.83 & $<0.1$ & 2.46 & 25.36 & 0.81 & & 2245 & $<1.6$ & 39.0 & 402 & 12.8 & 29.0 & \\
\hline $25 \mathrm{~A}$ & 100.06 & 11.40 & 0.79 & 423.71 & 0.58 & $<0.1$ & 6.94 & 61.74 & $<0.5$ & & 4831 & $<1.1$ & 79.1 & 704 & 5.7 & 6.6 & \\
\hline $26 \mathrm{E}$ & 102.95 & 13.75 & 1.00 & 156.9 & 0.95 & $<0.1$ & 3.16 & 34.49 & 1.28 & & 2157 & $<1.4$ & 43.4 & 474 & 17.6 & 13.1 & \\
\hline $26 \mathrm{~A}$ & 104.95 & 12.21 & 1.00 & 75.31 & 1.06 & $<0.1$ & 2.49 & 28.44 & 0.84 & & 919 & $<1.2$ & 30.4 & 347 & 10.3 & 12.9 & \\
\hline $27 \mathrm{G}$ & 107.05 & 15.02 & 1.00 & 342.38 & $<2$ & $<0.04$ & 4.71 & 39.20 & 1.07 & 611 & 5144 & $<0.60$ & 70.8 & 589 & 16.1 & $<30$ & 9174 \\
\hline $29 \mathrm{D}$ & 116.35 & 13.67 & 1.00 & 467.69 & 0.35 & $<0.04$ & 5.79 & 45.67 & & 49 & 6392 & $<0.55$ & 79.1 & 624 & nd & 4.8 & 676 \\
\hline $30 \mathrm{G}$ & 123.85 & 11.13 & 1.00 & 864.67 & $<0.4$ & 0.72 & 9.55 & 83.98 & 1.72 & 84 & 9623 & 8.01 & 106.3 & 935 & 19.1 & $<4.5$ & 936 \\
\hline $30 \mathrm{D}$ & 125.35 & 6.78 & 0.79 & 2179.7 & 0.82 & $<0.1$ & 17.81 & 89.94 & $<5$ & & 14774 & $<0.7$ & 120.7 & 610 & $<33.9$ & 5.6 & \\
\hline $30 \mathrm{~B}$ & 126.35 & 6.03 & 1.00 & 3168.8 & $<0.2$ & 42.80 & 25.28 & 39.80 & 0.91 & 83 & 19123 & 258.29 & 152.6 & 240 & $<5.5$ & $<1.2$ & 502 \\
\hline 30Bdup & 126.35 & 6.03 & 1.00 & 3439.5 & $<0.2$ & 31.17 & 26.88 & 43.8 & 3.49 & & 20757 & 188.11 & 162.2 & 264 & $<21.1$ & $<1.2$ & \\
\hline $31 \mathrm{G}$ & 129.15 & 6.62 & 1.00 & 3932.5 & $<0.2$ & \begin{tabular}{|l|}
27.78 \\
\end{tabular} & 32.52 & 23.92 & $<1$ & 66 & 26033 & 183.90 & 215.3 & 158 & $<6.6$ & $<1.3$ & 436 \\
\hline $31 \mathrm{~A}$ & 132.15 & 6.36 & 1.00 & 3195.7 & $<0.5$ & $<1$ & 30.57 & 10.95 & $<5$ & & 20329 & $<6$ & 194.5 & 70 & $<31.8$ & $<3.2$ & \\
\hline $32 \mathrm{D}$ & 134.25 & 11.80 & 0.84 & 811.76 & 1.16 & $<1$ & 9.94 & 6.86 & $<5$ & & 9577 & $<12$ & 117.3 & 81 & $<59.0$ & 13.7 & \\
\hline $32 \mathrm{~A}$ & 135.80 & 5.79 & 1.00 & 2775.7 & $<0.2$ & 12.86 & 26.98 & 14.88 & $<1$ & 38 & 16064 & 74.43 & 156.1 & 86 & $<5.8$ & $<1.2$ & 221 \\
\hline $33 \mathrm{G}$ & 137.65 & 6.43 & 1.00 & 2733.6 & $<0.2$ & 9.34 & 26.41 & 10.33 & $<1$ & 72 & 17588 & 60.09 & 169.9 & 66 & $<6.4$ & $<1.3$ & 462 \\
\hline $33 \mathrm{~B}$ & 140.15 & 6.45 & 1.00 & 2738.1 & $<0.2$ & 13.15 & 25.44 & 11.05 & $<1$ & 34 & 17672 & 84.87 & 164.2 & 71 & $<6.5$ & $<1.3$ & 219 \\
\hline $35 \mathrm{~K}$ & 141.05 & 8.72 & 1.00 & 2593.9 & $<0.2$ & \begin{tabular}{|l|} 
\\
\end{tabular} & 23.87 & 8.49 & $<1$ & 43 & 22623 & 74.22 & 208.2 & 74 & $<8.7$ & $<1.7$ & 376 \\
\hline $35 \mathrm{C}$ & 144.85 & 6.15 & 1.00 & 1552.5 & $<0.2$ & 2.01 & 15.77 & 10.75 & $<1$ & 134 & 9550 & 12.36 & 97.0 & 66 & $<6.2$ & $<1.2$ & 826 \\
\hline $35 \mathrm{~A}$ & 145.85 & 3.89 & 1.00 & 2454.2 & $<0.2$ & 4.16 & 26.28 & 12.40 & 8.12 & 41 & 9544 & 16.18 & 102.2 & 48 & $<31.6$ & $<0.8$ & 160 \\
\hline $36 \mathrm{D}$ & 147.45 & 10.20 & 1.00 & 761.89 & 0.75 & $<1$ & 9.86 & 7.66 & $<5$ & & 7774 & $<10$ & 100.6 & 78 & $<51.0$ & 7.7 & \\
\hline $36 \mathrm{~B}$ & 148.45 & 4.35 & 1.00 & 1385.4 & $<0.2$ & 0.78 & 17.42 & 19.82 & $<1$ & 39 & 6033 & 3.40 & 75.9 & 86 & $<4.4$ & $<0.9$ & 171 \\
\hline 37D & 149.65 & 7.72 & 1.00 & 2140 & $<0.2$ & 2.32 & 21.31 & 11.87 & $<1$ & 59 & 16524 & 17.91 & 164.5 & 92 & $<7.7$ & $<1.5$ & 452 \\
\hline $38 \mathrm{G}$ & 152.10 & 4.32 & 1.00 & 408.97 & 1.28 & $<1$ & 11.33 & 17.88 & $<5$ & & 1768 & $<4$ & 49.0 & 77 & $<21.6$ & 5.5 & \\
\hline $38 \mathrm{E}$ & 153.10 & 7.11 & 1.00 & 241.57 & $<2$ & $<0.04$ & 7.39 & 17.71 & 0.22 & 60 & 1718 & $<0.28$ & 52.6 & 126 & $<1.6$ & $<14$ & 425 \\
\hline
\end{tabular}




\begin{tabular}{|c|c|c|c|c|c|c|c|c|c|c|c|c|c|c|c|c|c|}
\hline \multirow{2}{*}{$\begin{array}{c}\text { Sample } \\
\text { ID }\end{array}$} & \multirow{2}{*}{$\begin{array}{l}\text { Depth } \\
\text { (ft bgs) }\end{array}$} & \multirow[b]{2}{*}{ Dil. Fac. } & \multirow{2}{*}{$\begin{array}{c}\text { water:soil } \\
\text { Ratio }\end{array}$} & \multicolumn{7}{|c|}{ 1:1 Extracts Concentrations ( milligrams per liter) } & \multicolumn{7}{|c|}{ Dilution-Corrected Porewater Concentrations (milligrams per liter) } \\
\hline & & & & Nitrate & Fluoride & Nitrite & Chloride & Sulfate & Phosphate & Alk & Nitrate & Nitrite & Chloride & Sulfate & Phosphate & Fluoride & Alk \\
\hline $38 \mathrm{E}-\mathrm{dup}$ & 153.10 & 7.11 & 1.00 & 216.81 & $<2$ & $<0.04$ & 6.91 & 14.34 & 0.18 & & 1543 & $<0.28$ & 49.2 & 102 & $<1.3$ & $<14$ & \\
\hline $39 \mathrm{D}$ & 155.35 & 9.49 & 0.82 & 292.81 & 0.31 & $<0.1$ & 7.61 & 14.22 & $<0.5$ & & 2779 & $<0.9$ & 72.2 & 135 & $<4.7$ & 2.9 & \\
\hline $39 \mathrm{C}$ & 155.85 & 12.83 & 1.00 & 90.08 & 0.30 & $<0.04$ & 3.81 & 11.03 & $<1$ & 76 & 1156 & $<0.51$ & 48.9 & 142 & $<12.8$ & 3.85 & 971 \\
\hline 39C-dup & 155.85 & 12.82 & 1.00 & 83.59 & 0.30 & $<0.04$ & 3.59 & 11.25 & $<1$ & & 1072 & $<0.51$ & 46.0 & 144 & $<12.8$ & 12.82 & \\
\hline $40 \mathrm{~B}$ & 157.75 & 20.49 & 1.00 & 3.56 & 0.32 & $<0.04$ & 1.34 & 5.98 & $<0.1$ & & 73 & $<0.82$ & 27.5 & 123 & $<2.0$ & 6.56 & \\
\hline 40 & 158.65 & 10.96 & 1.38 & 24.93 & 0.56 & $<0.1$ & 2.12 & 38.84 & $<0.5$ & 74 & 273 & $<1.1$ & 23.2 & 426 & $<5.5$ & 6.1 & 815 \\
\hline $17 \mathrm{SK}$ & 159.75 & 11.92 & 1.00 & 32.24 & 0.84 & $<0.1$ & 4.25 & 14.47 & $<0.5$ & & 384 & $<1.2$ & 50.7 & 173 & $<6.0$ & 10.0 & \\
\hline 18SKA & 161 & 14.68 & 1.01 & 23.02 & 2.3 & $<0.1$ & 3.03 & 17.31 & $<5$ & & 338 & $<1.5$ & 44.5 & 254 & $<73.4$ & 33.8 & \\
\hline 18SKB & 161.00 & 19.78 & 1.04 & 28.29 & $<0.1$ & 2.72 & 0.94 & 16.99 & $<0.5$ & & 560 & 54 & 18.6 & 336 & $<9.9$ & $<2.0$ & \\
\hline 18SKC & 166.50 & 34.13 & 1.01 & 17.19 & $<0.1$ & 3.75 & 1 & 12.96 & $<0.5$ & & 587 & 128 & 34.1 & 442 & $<17.1$ & $<3.4$ & \\
\hline 19SKA & 166.50 & 74.25 & 1.00 & 11.36 & 1.01 & $<0.1$ & 1.6 & 10.29 & $<0.5$ & & 843 & $<7.4$ & 118.8 & 764 & $<37.1$ & 75.0 & \\
\hline 19SKB & 176.00 & 80.64 & 1.00 & 5.65 & 0.44 & $<0.1$ & 1.27 & 11.28 & $<0.5$ & & 456 & $<8.1$ & 102.4 & 910 & $<40.3$ & 35.5 & \\
\hline $41 \mathrm{~A}$ & 184.45 & 25.37 & 1.00 & 6.53 & 0.37 & $<0.04$ & 1.28 & 5.17 & $<0.1$ & 440 & 166 & $<1.01$ & 32.5 & 131 & $<2.5$ & 9.4 & 11170 \\
\hline 19SKC & 185 & 114.65 & 1.01 & 3.87 & 0.42 & $<0.1$ & 1.36 & 6.5 & $<0.5$ & & 444 & $<11.5$ & 155.9 & 745 & $<57.3$ & 48.2 & \\
\hline 20SKA & 185 & 78.06 & 1.00 & 0.78 & 0.65 & $<0.1$ & 1.45 & 4.13 & $<0.5$ & & 61 & $<7.8$ & 113.2 & 322 & $<39.0$ & 50.7 & \\
\hline 20SKB & 189.5 & 82.45 & 0.94 & 3.13 & 0.58 & $<0.1$ & 2.51 & 5.09 & $<0.5$ & & 258 & $<8.2$ & 206.9 & 420 & $<41.2$ & 47.8 & \\
\hline 42 & 194.55 & 17.10 & 1.00 & 1.14 & 0.65 & $<0.04$ & 2.00 & 7.38 & $<0.1$ & 57 & 19 & $<0.68$ & 34.2 & 126 & $<1.7$ & 11.1 & 966 \\
\hline $43 \mathrm{~B}$ & 204.50 & 16.25 & 1.00 & 76.79 & 0.36 & $<0.04$ & 3.59 & 7.68 & 0.13 & 45 & 1248 & $<0.65$ & 58.3 & 125 & $<2.1$ & 5.85 & 736 \\
\hline $43 \mathrm{~A}$ & 205.15 & 41.39 & 1.00 & 25.29 & 0.53 & $<0.1$ & 1.47 & 3.68 & $<0.5$ & & 1047 & $<4.1$ & 60.8 & 152 & $<20.7$ & 21.9 & \\
\hline \multicolumn{18}{|c|}{$\begin{array}{l}\text { *Alk = alkalinity as milligrams per liter of } \mathrm{CaCO}_{3} \text {; alkalinity values in bold red type are anomalous due to malfunction of laboratory equipment } \\
\text { Empty spaces are analytes that were not determined/measured } \\
\text { Values in light blue shading represent water extracts that were inadvertently run at a ratio of less than } 1: 1 \text { and analytes reported may be biased high in relationship } \\
\text { to the correct } 1: 1 \text { extract samples } \\
\text { Values in orange shading represent water extracts that were inadvertently run at a ratio greater than } 1: 1 \text { and analytes reported may be biased low in relationship } \\
\text { to the correct } 1: 1 \text { extract samples } \\
\text { dup = Duplicate sample }\end{array}$} \\
\hline
\end{tabular}


Table 4.5 Comparison of Dilution-corrected and Actual Porewater Anion Concentrations in Vadose Zone Sediment from Borehole 299-W23-19

\begin{tabular}{|c|c|c|c|c|c|c|c|c|c|}
\hline \multirow{2}{*}{$\begin{array}{c}\text { Sample } \\
\text { ID }\end{array}$} & \multirow{2}{*}{$\begin{array}{l}\text { Depth } \\
(\text { ft bgs })^{\mathrm{a}}\end{array}$} & \multirow[b]{2}{*}{ Dil. Fac. } & \multicolumn{7}{|c|}{ Dilution-corrected Porewater Concentration (milligrams per liter) } \\
\hline & & & Nitrate & Nitrite & Chloride & Sulfate & Phosphate & Fluoride & Alk ${ }^{*}$ \\
\hline $17 \mathrm{~F}$ & 63.75 & 8.53 & 75 & $<0.3$ & 5.1 & 135 & 1.6 & 6.1 & 664 \\
\hline 17DE-ufa & 64.75 & 1.00 & 131 & $<0.8$ & 6.7 & 151 & $<2$ & 0.6 & 183 \\
\hline 19D & \multirow{2}{*}{75.25} & 9.61 & 3088 & $<1.0$ & 44.2 & 272 & 8.5 & 14.6 & \\
\hline 19D-ufa & & 1.00 & & & & & & & \\
\hline $20 \mathrm{E}$ & \multirow{2}{*}{79.75} & 7.79 & 2150 & $<0.8$ & 26.7 & 211 & 10.7 & 29.5 & \\
\hline 20E-ufa & & 1.00 & & & & & & & \\
\hline $35 \mathrm{~K}$ & 141.05 & 8.72 & 22623 & 74.2 & 208.2 & 74 & $<8.7$ & $<1.7$ & 376 \\
\hline 35IH-ufa & 141.85 & 1.00 & 18461 & 65.9 & 171.7 & 45 & nd & $<4$ & 52 \\
\hline $35 \mathrm{~F}-\mathrm{ufa}$ & 143.35 & 1.00 & 15572 & 58.4 & 148.3 & 33 & 232 & $<2$ & \\
\hline $35 \mathrm{C}$ & 144.85 & 6.15 & 9550 & 12.4 & 97.0 & 66 & $<6.2$ & $<1.2$ & 826 \\
\hline 38A-ufa & 155.1 & 1.00 & 849.4 & 0.4 & 58.2 & 102 & 8.5 & $<0.4$ & 153 \\
\hline 39D & 155.35 & 9.49 & 2779 & $<0.9$ & 72.2 & 135 & $<4.7$ & 2.9 & \\
\hline \multicolumn{10}{|c|}{$\begin{array}{l}\text { (a) multiply by } 0.3048 \text { to convert to meters } \\
\text { ufa = ultracentrifuge } \\
* \text { Alk = alkalinity as milligrams per liter of calcium carbonate } \\
\text { nd = not detected } \\
\text { na = not analyzed }\end{array}$} \\
\hline
\end{tabular}



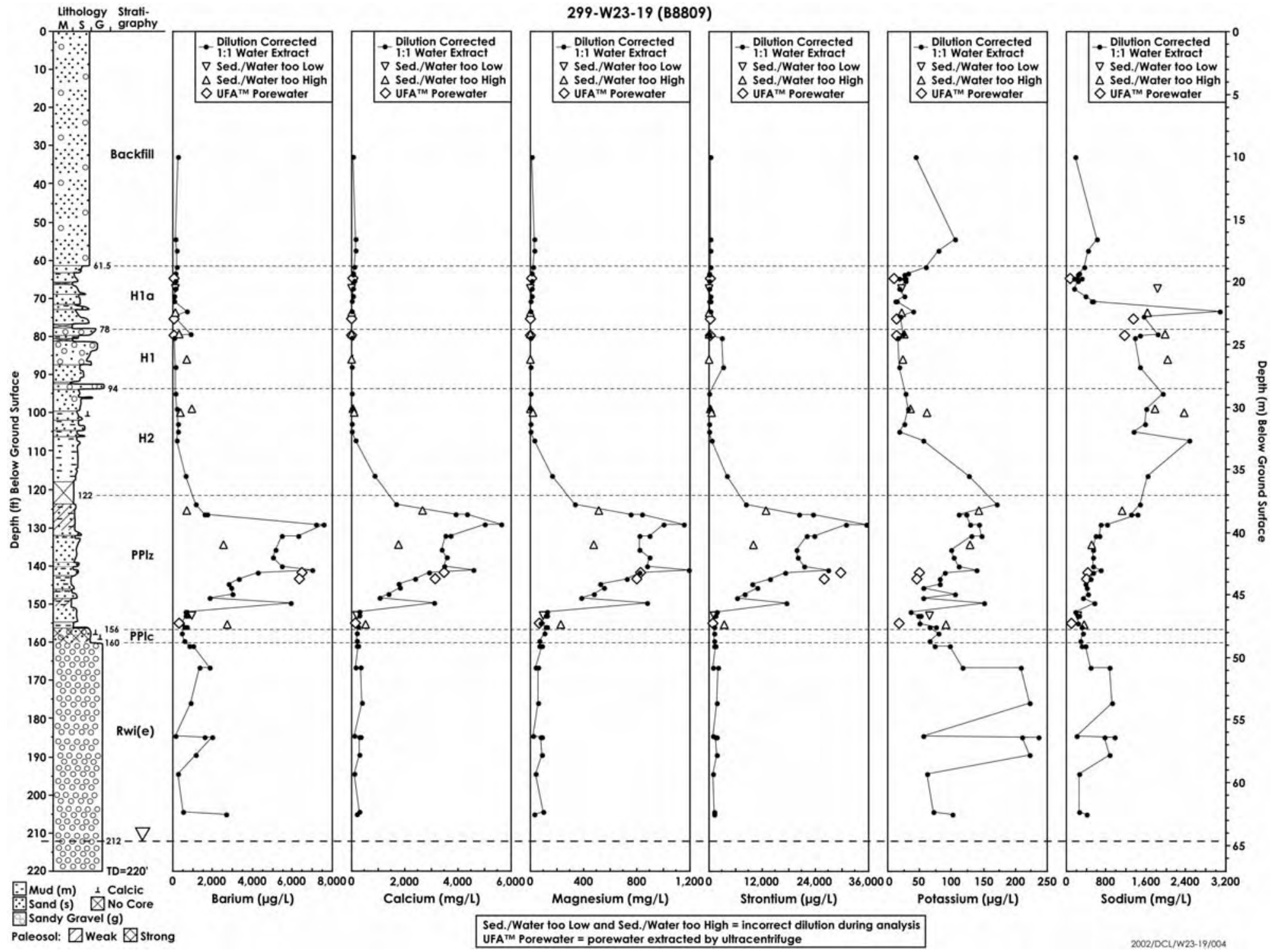

Figure 4.4 Cations Calculated [from sediment:water extracts] and Actual Porewaters for Borehole 299-W23-19 Sediment 
Table 4. 6 Calculated Porewater Cation Composition from Water Extracts from Vadose Zone Sediment from 299-W23-19 Borehole

\begin{tabular}{|c|c|c|c|c|c|c|c|c|c|c|c|c|c|c|}
\hline $\begin{array}{c}\text { Sample } \\
\text { ID }\end{array}$ & $\begin{array}{l}\text { Depth } \\
(\text { ft bgs })^{\mathrm{a}}\end{array}$ & Dil. Fac. & $\begin{array}{c}\text { Water:soil } \\
\text { Ratio }\end{array}$ & $\begin{array}{c}\text { Al } \\
(\mathbf{u g} / \mathrm{L})\end{array}$ & $\begin{array}{c}\text { Ba } \\
\text { (ug/L) }\end{array}$ & $\begin{array}{c}\text { Ca } \\
\text { (milligram } \\
\text { s per liter) }\end{array}$ & $\begin{array}{c}\text { Fe } \\
(\text { ug/L) }\end{array}$ & \begin{tabular}{|c} 
K \\
(milligra \\
ms per \\
liter) \\
\end{tabular} & $\begin{array}{c}\text { Mg } \\
\text { (milligrams } \\
\text { per liter) }\end{array}$ & $\begin{array}{c}\mathrm{Na} \\
\text { (milligrams } \\
\text { per liter) }\end{array}$ & \begin{tabular}{|c|}
$\mathbf{S i}$ \\
(milligrams \\
per liter)
\end{tabular} & $\begin{array}{c}\mathrm{Sr} \\
(\mathbf{u g} / \mathbf{L})\end{array}$ & $\begin{array}{c}\mathrm{SO}_{4} \\
\text { (milligrams } \\
\text { per liter) }\end{array}$ & $\begin{array}{c}\text { Mn } \\
(\mathbf{u g} / \mathrm{L}) \\
\end{array}$ \\
\hline $09 \mathrm{E}$ & 32.95 & 9.85 & 1.00 & 341 & 248 & 66 & 335 & 44 & 12 & 185 & 189 & 380 & 19 & 10 \\
\hline $15 \mathrm{~A}$ & 54.50 & 17.13 & 1.00 & 327 & 153 & 134 & 1097 & 105 & 27 & 611 & 277 & 319 & 226 & 73 \\
\hline $16 \mathrm{~A}$ & 57.45 & 15.82 & 1.00 & 51 & 197 & 143 & 680 & 79 & 30 & 432 & 253 & 186 & 266 & 38 \\
\hline $17 \mathrm{~J}$ & 61.75 & 18.84 & 1.00 & 548 & 170 & 101 & 1093 & 60 & 21 & 359 & 237 & 297 & 163 & 41 \\
\hline $17 \mathrm{G}$ & 63.25 & 11.13 & 1.00 & 85 & 156 & 71 & 746 & 32 & 14 & 250 & 172 & 142 & 132 & 21 \\
\hline $17 \mathrm{~F}$ & 63.75 & 8.53 & 1.00 & 211 & 85 & 65 & 1416 & 25 & 13 & 243 & 154 & 135 & 125 & 32 \\
\hline 17F-dup & 64.75 & 8.56 & 1.01 & 317 & 101 & 63 & 592 & 25 & 12 & 215 & 159 & 375 & 105 & 11 \\
\hline 17DE-aft & 64.75 & 10.70 & 0.96 & 164 & 161 & 72 & 308 & 27 & 14 & 316 & 198 & 123 & 126 & 12 \\
\hline $17 \mathrm{DE}$ & 64.75 & 5.05 & 1.00 & $<250$ & 65 & 62 & 71 & 19 & 8 & 164 & 94 & 315 & 157 & 2 \\
\hline $17 \mathrm{C}$ & 65.25 & 9.03 & 1.00 & $<250$ & 144 & 77 & 824 & 29 & 15 & 231 & 150 & 123 & 178 & 22 \\
\hline $18 \mathrm{I}$ & 67.45 & 5.74 & 1.00 & 2045 & 103 & 62 & 4160 & 19 & 12 & 163 & 103 & 154 & 146 & 64 \\
\hline $18 \mathrm{E}$ & 69.45 & 10.19 & 1.00 & 182 & 81 & 36 & 526 & 26 & 6 & 373 & 168 & 205 & 126 & 16 \\
\hline $18 \mathrm{CB}$ & 70.70 & 6.56 & 1.00 & 1882 & 72 & 8 & 4559 & 11 & 1 & 537 & 119 & 206 & 134 & 84 \\
\hline 18CB-dup & 70.70 & 6.56 & 1.00 & 2896 & 90 & 10 & 7919 & 14 & 2 & 518 & 124 & 54 & 147 & 140 \\
\hline $19 \mathrm{H}$ & 73.25 & 27.70 & 1.00 & 3057 & 692 & 25 & 5361 & 39 & 3 & 3081 & 372 & 192 & 458 & 131 \\
\hline $19 \mathrm{G}$ & 73.75 & 7.49 & 0.86 & $<250$ & 112 & 18 & 1477 & 23 & 2 & 1632 & 112 & 130 & 311 & 54 \\
\hline $19 \mathrm{E}$ & 74.75 & 7.09 & 1.00 & 442 & 92 & 17 & 2543 & 21 & 2 & 1542 & 113 & 36 & 321 & 96 \\
\hline 19D & 75.25 & 9.61 & 1.18 & 1554 & 154 & 12 & 3340 & 22 & 1 & 1825 & 148 & 98 & 273 & 67 \\
\hline $20 \mathrm{~F}$ & 79.25 & 9.47 & 0.76 & 529 & 332 & 10 & 1694 & 25 & 1 & 1977 & 148 & 86 & 301 & 39 \\
\hline 20F-dup & 79.25 & 9.38 & 1.04 & 1267 & 910 & 12 & 5357 & 23 & 1 & 1841 & 156 & 122 & 265 & 126 \\
\hline $20 \mathrm{E}$ & 79.75 & 7.79 & 1.00 & 1166 & 156 & 9 & 9829 & 19 & 1 & 1472 & 125 & 74 & 214 & 146 \\
\hline $20 \mathrm{D}$ & 80.25 & 6.00 & 1.00 & 1210 & 78 & 6 & 6094 & 15 & 1 & 1365 & 97 & 2998 & 219 & 115 \\
\hline
\end{tabular}




\begin{tabular}{|c|c|c|c|c|c|c|c|c|c|c|c|c|c|c|}
\hline $\begin{array}{c}\text { Sample } \\
\text { ID }\end{array}$ & $\begin{array}{l}\text { Depth } \\
(\text { ft bgs) })^{a}\end{array}$ & Dil. Fac. & Water:soil & $\begin{array}{c}\text { Al } \\
(\mathrm{ug} / \mathrm{L}) \\
\end{array}$ & $\begin{array}{c}\text { Ba } \\
\text { (ug/L) } \\
\end{array}$ & \begin{tabular}{|c|} 
Ca \\
(milligram \\
s per liter) \\
\end{tabular} & (ug/L) & $\begin{array}{c}\mathrm{K} \\
\text { (milligra } \\
\text { ms per } \\
\text { liter) }\end{array}$ & \begin{tabular}{|c|} 
Mg \\
(milligrams \\
per liter $)$ \\
\end{tabular} & \begin{tabular}{|c|}
$\mathbf{N a}$ \\
(milligrams \\
per liter)
\end{tabular} & $\begin{array}{c}\text { Si } \\
\text { (milligrams } \\
\text { per liter) } \\
\end{array}$ & $\begin{array}{c}\mathrm{Sr} \\
(\mathbf{u g} / \mathbf{L})\end{array}$ & \begin{tabular}{|c|}
$\mathrm{SO}_{4}$ \\
(milligrams \\
per liter) \\
\end{tabular} & $\begin{array}{c}\text { Mn } \\
(\mathrm{ug} / \mathrm{L}) \\
\end{array}$ \\
\hline $21 \mathrm{~A}$ & 86.00 & 13.58 & 0.61 & 2555 & 693 & 10 & 2660 & 24 & 2 & 2046 & 254 & 106 & 190 & 76 \\
\hline $22 \mathrm{D}$ & 88.10 & 6.07 & 1.00 & 490 & 103 & 7 & 3413 & 18 & 1 & 1476 & 63 & 3033 & 261 & 85 \\
\hline $24 \mathrm{~A}$ & 95.00 & 10.82 & 1.00 & 2038 & 141 & 17 & 3773 & 28 & 3 & 1921 & 103 & 140 & 378 & 61 \\
\hline $25 \mathrm{~B}$ & 99.06 & 14.17 & 0.81 & 747 & 935 & 29 & 365 & 35 & 4 & 1781 & 148 & 210 & 468 & 11 \\
\hline 25B-dup & 99.06 & 15.85 & 0.97 & 888 & 206 & 19 & 396 & 31 & 3 & 1612 & 173 & 128 & 396 & 8 \\
\hline $25 \mathrm{~A}$ & 100.06 & 11.40 & 0.79 & 811 & 365 & 103 & 899 & 62 & 16 & 2351 & 134 & 651 & 697 & 13 \\
\hline $26 \mathrm{E}$ & 102.95 & 13.75 & 1.00 & $<1000$ & 289 & 16 & 1142 & 26 & 2 & 1589 & 127 & 129 & 454 & 39 \\
\hline $26 \mathrm{~A}$ & 104.95 & 12.21 & 1.00 & 890 & 281 & 9 & 448 & 18 & 1 & 1360 & 111 & 74 & 322 & 18 \\
\hline $27 \mathrm{G}$ & 107.05 & 15.02 & 1.00 & $<1000$ & 210 & 162 & 480 & 57 & 32 & 2457 & 181 & 509 & 570 & 12 \\
\hline $29 \mathrm{D}$ & 116.35 & 13.67 & 1.00 & 374 & 670 & 844 & 107 & 128 & 158 & 1619 & 191 & 4116 & 511 & 52 \\
\hline $30 \mathrm{G}$ & 123.85 & 11.13 & 1.00 & $<500$ & 1157 & 1670 & 23 & 170 & 338 & 1481 & 122 & 8332 & 798 & 428 \\
\hline $30 \mathrm{D}$ & 125.35 & 6.78 & 0.79 & $<500$ & 705 & 2653 & 30 & 142 & 518 & 1111 & 74 & 12930 & 587 & 296 \\
\hline $30 \mathrm{~B}$ & 126.35 & 6.03 & 1.00 & $<500$ & 1575 & 3906 & 1773 & 112 & 749 & 1289 & 67 & 20317 & 249 & 818 \\
\hline 30B-dup & 126.35 & 6.03 & 1.00 & $<500$ & 1730 & 4349 & 48 & 122 & 835 & 1414 & 71 & 23461 & 268 & 832 \\
\hline $31 \mathrm{G}-1 \mathrm{st}$ & 129.15 & 6.62 & 1.00 & $<500$ & 7202 & 5618 & $<50$ & 129 & 1150 & 818 & 71 & 35522 & 93 & 883 \\
\hline $31 \mathrm{G}$ & 129.15 & 5.15 & 1.00 & $<500$ & 7549 & 5000 & 12 & 142 & 999 & 680 & 51 & 30836 & 82 & 728 \\
\hline $31 \mathrm{~A}$ & 132.15 & 6.36 & 1.00 & $<500$ & 6285 & 3740 & 63 & 147 & 892 & 655 & 71 & 23715 & 71 & 335 \\
\hline $31 \mathrm{~A}$ & 132.15 & 6.35 & 1.00 & $<500$ & 5493 & 3522 & 28 & 132 & 819 & 589 & 66 & 22157 & 55 & 301 \\
\hline $32 \mathrm{D}$ & 134.25 & 11.80 & 0.84 & $<500$ & 2560 & 1788 & 88 & 128 & 472 & 513 & 127 & 9996 & 77 & 70 \\
\hline $32 \mathrm{~A}$ & 135.80 & 5.79 & 1.00 & $<500$ & 5168 & 3374 & 1 & 100 & 823 & 534 & 60 & 19626 & 69 & 250 \\
\hline $33 \mathrm{G}$ & 137.65 & 6.43 & 1.00 & $<500$ & 5044 & 3578 & $<50$ & 104 & 893 & 523 & 64 & 20064 & 65 & 306 \\
\hline $33 \mathrm{~B}$ & 140.15 & 6.45 & 1.00 & $<500$ & 5486 & 3498 & 6 & 111 & 876 & 528 & 65 & 21405 & 75 & 253 \\
\hline $35 \mathrm{~K}$ & "141.05" & 8.72 & 1.00 & $<500$ & 7003 & 4552 & 35 & 139 & 1191 & 689 & 88 & 26880 & 84 & 450 \\
\hline $35 \mathrm{IH}-\mathrm{aft}$ & 141.85 & 6.30 & 1.00 & $<500$ & 4275 & 2885 & 151 & 89 & 817 & 533 & 64 & 17040 & 73 & 30 \\
\hline $35 \mathrm{~F}-\mathrm{aft}$ & 143.35 & 7.18 & 1.04 & $<500$ & 3275 & 2361 & 151 & 82 & 723 & 478 & 74 & 13667 & 87 & 28 \\
\hline
\end{tabular}




\begin{tabular}{|c|c|c|c|c|c|c|c|c|c|c|c|c|c|c|}
\hline $\begin{array}{c}\text { Sample } \\
\text { ID }\end{array}$ & $\begin{array}{l}\text { Depth } \\
\text { (ft bgs) }\end{array}$ & Dil. Fac. & $\begin{array}{c}\text { Water:soil } \\
\text { Ratio }\end{array}$ & $\begin{array}{c}\text { Al } \\
(\mathbf{u g} / \mathbf{L})\end{array}$ & $\begin{array}{c}\text { Ba } \\
(\mathbf{u g} / \mathbf{L})\end{array}$ & \begin{tabular}{|c|} 
Ca \\
(milligram \\
s per liter) \\
\end{tabular} & (ug/L) & $\begin{array}{c}\text { K } \\
\text { (milligra } \\
\text { ms per } \\
\text { liter) }\end{array}$ & $\begin{array}{c}\text { Mg } \\
\text { (milligrams } \\
\text { per liter) }\end{array}$ & \begin{tabular}{|c|} 
Na \\
(milligrams \\
per liter)
\end{tabular} & $\begin{array}{c}\text { Si } \\
\text { (milligrams } \\
\text { per liter) }\end{array}$ & $\mid \begin{array}{c}\mathrm{Sr} \\
(\mathbf{u g} / \mathbf{L})\end{array}$ & $\begin{array}{c}\mathrm{SO}_{4} \\
\text { (milligrams } \\
\text { per liter) }\end{array}$ & $\begin{array}{c}\text { Mn } \\
(\mathbf{u g} / \mathrm{L})\end{array}$ \\
\hline $35 \mathrm{C}$ & 144.85 & 6.15 & 1.00 & $<500$ & 2805 & 1743 & 18 & 81 & 521 & 391 & 55 & 9738 & 73 & 134 \\
\hline $35 \mathrm{~A}$ & 145.85 & 3.89 & 1.00 & $<500$ & 2921 & 1811 & 16 & 57 & 554 & 412 & 43 & 10833 & 55 & 9 \\
\hline $36 \mathrm{D}$ & 147.45 & 10.20 & 1.00 & $<500$ & 2959 & 1388 & 81 & 106 & 476 & 430 & 85 & 8160 & 76 & 29 \\
\hline $36 \mathrm{~B}$ & 148.45 & 4.35 & 1.00 & $<500$ & 1859 & 1047 & 30 & 56 & 377 & 341 & 47 & 6238 & 71 & 6 \\
\hline $37 \mathrm{D}$ & 149.65 & 7.72 & 1.00 & $<500$ & 5891 & 3086 & $<50$ & 150 & 873 & 554 & 74 & 17468 & 95 & 2496 \\
\hline $38 \mathrm{G}$ & 152.10 & 4.32 & 1.00 & $<500$ & 661 & 282 & 25 & 36 & 122 & 192 & 48 & 1753 & 80 & 2 \\
\hline $38 \mathrm{G}$ & 152.10 & 4.32 & 1.00 & $<500$ & 709 & 300 & 55 & 37 & 129 & 175 & 47 & 1845 & 76 & 3 \\
\hline $38 \mathrm{E}$ & 153.10 & 7.11 & 1.00 & 93 & 783 & 268 & 138 & 49 & 124 & 257 & 70 & 1472 & 100 & 8 \\
\hline 38E-dup & 153.10 & 7.11 & 1.00 & 162 & 665 & 252 & 25 & 53 & 117 & 237 & 72 & 1454 & 95 & 27 \\
\hline 38A-aft & 155.10 & 10.92 & 1.00 & $<500$ & 448 & 152 & 680 & 50 & 74 & 242 & 162 & 513 & 151 & 30 \\
\hline $39 \mathrm{D}$ & 155.35 & 9.49 & 0.82 & $<500$ & 2743 & 526 & 97 & 91 & 226 & 352 & 111 & 3365 & 179 & 66 \\
\hline $39 \mathrm{C}$ & 155.85 & 12.83 & 1.00 & 151 & 706 & 250 & 285 & 75 & 122 & 318 & 222 & 1231 & 139 & 9 \\
\hline 39C-dup & 155.85 & 12.82 & 1.00 & $<500$ & 554 & 237 & $<50$ & 66 & 115 & 309 & 206 & 1088 & 131 & 12 \\
\hline $40 \mathrm{~B}$ & 157.75 & 20.49 & 1.00 & 156 & 451 & 218 & 746 & 79 & 104 & 328 & 323 & 1210 & 126 & 30 \\
\hline 40 & 158.65 & 10.96 & 1.38 & $<500$ & 932 & 210 & 65 & 66 & 92 & 226 & 189 & 1268 & 471 & 6 \\
\hline $17 \mathrm{SK}$ & 159.75 & 11.92 & 1.00 & $<500$ & 596 & 170 & 89 & 65 & 67 & 273 & 195 & 1083 & 188 & 45 \\
\hline 18SKA & 161 & 14.68 & 1.01 & $<500$ & 822 & 180 & 46 & 74 & 66 & 317 & 222 & 1088 & 267 & 45 \\
\hline $18 \mathrm{SKB}$ & 161.00 & 19.78 & 1.04 & $<500$ & 1009 & 235 & 170 & 97 & 90 & 392 & 278 & 1371 & 368 & 62 \\
\hline 18SKC & 166.50 & 34.13 & 1.01 & $<500$ & 1843 & 157 & 222 & 118 & 42 & 483 & 239 & 1018 & 480 & 22 \\
\hline 19SKA & 166.50 & 74.25 & 1.00 & $<500$ & 1336 & 343 & 379 & 209 & 62 & 857 & 626 & 2036 & 821 & 29 \\
\hline 19SKB & 176.00 & 80.64 & 1.00 & $<500$ & 887 & 372 & 419 & 223 & 56 & 904 & 814 & 1870 & 878 & 79 \\
\hline $41 \mathrm{~A}$ & 184.45 & 25.37 & 1.00 & 297 & 127 & 76 & 1091 & 55 & 24 & 213 & 274 & 845 & 128 & 37 \\
\hline 19SKC & 185 & 114.65 & 1.01 & $<500$ & 1605 & 315 & 757 & 235 & 87 & 965 & 951 & 1873 & 796 & 83 \\
\hline $20 \mathrm{SKA}$ & 185 & 78.06 & 1.00 & $<500$ & 1952 & 294 & 1226 & 211 & 74 & 776 & 606 & 1558 & 342 & 310 \\
\hline $20 \mathrm{SKB}$ & 189.5 & 82.45 & 0.94 & $<500$ & 1154 & 308 & 618 & 223 & 83 & 877 & 700 & 1772 & 501 & 379 \\
\hline
\end{tabular}




\begin{tabular}{|c|c|c|c|c|c|c|c|c|c|c|c|c|c|c|}
\hline $\begin{array}{c}\text { Sample } \\
\text { ID }\end{array}$ & Depth & Dil. Fac. & Water:soil & $\begin{array}{c}\text { Al } \\
\text { (ug/L) } \\
\end{array}$ & $\begin{array}{c}\text { Ba } \\
(\mathrm{ug} / \mathrm{L}) \\
\end{array}$ & $\begin{array}{c}\text { Ca } \\
\text { (milligram } \\
\text { s per liter) } \\
\end{array}$ & $\begin{array}{c}\mathrm{Fe} \\
(\mathrm{ug} / \mathrm{L}) \\
\end{array}$ & $\begin{array}{c}\mathbf{K} \\
\text { (milligra } \\
\text { ms per } \\
\text { liter) }\end{array}$ & $\begin{array}{c}\text { Mg } \\
\text { (milligrams } \\
\text { per liter) }\end{array}$ & \begin{tabular}{|c|}
$\mathrm{Na}$ \\
(milligrams \\
per liter)
\end{tabular} & $\begin{array}{c}\text { Si } \\
\text { (milligrams } \\
\text { per liter) }\end{array}$ & $\begin{array}{c}\mathrm{Sr} \\
\\
(\mathrm{ug} / \mathrm{L})\end{array}$ & $\begin{array}{c}\mathrm{SO}_{4} \\
\text { (milligrams } \\
\text { per liter) }\end{array}$ & $(\mathrm{ug} / \mathrm{L})$ \\
\hline 42 & 194.55 & 17.10 & 1.00 & 15095 & 257 & 109 & 15152 & 61 & 39 & 250 & 245 & 990 & 144 & 394 \\
\hline $43 B$ & 204.50 & 16.25 & 1.00 & 421 & 487 & 280 & 487 & 72 & 91 & 267 & 156 & 1268 & 120 & 123 \\
\hline $43 \mathrm{~A}$ & 205.15 & 41.39 & 1.00 & $<500$ & 2691 & 173 & 435 & 101 & 30 & 411 & 421 & 1064 & 214 & 28 \\
\hline \multicolumn{15}{|c|}{$\begin{array}{l}\text { (a) multiply by } 0.3048 \text { to convert to meters } \\
\text { dup = a duplicate water extract on a separate aliquot of sediment } \\
\text { aft = water extract AFTER ultracentrifugation to remove actual porewater } \\
\text { SK\# = sediment from air-rotary sock not from core barrel. } \\
\text { Extracts with incorrect water-to-sediment ratios are highlighted in orange and blue } \\
\text { Note that some analytes are reported as } \mu \mathrm{g} / \mathrm{L} \text { and others milligrams per liter }\end{array}$} \\
\hline
\end{tabular}


than do the dilution-corrected water extracts; both porewaters have about the same calcium concentration in the upper Plio-Pleistocene unit. Finally, all the actual porewater samples have larger strontium concentrations than do the comparable dilution-corrected porewater samples. This suggests that diluting the existing porewater with deionized water during extraction causes strontium to adsorb to the sediment to a greater degree than when the porewaters are more concentrated. The agreement between the concentrations of potassium and magnesium in the actual and the dilution-corrected porewaters is quite good.

The depth distribution of the major cations appears to reflect a traditional ion-exchange process whereby the high concentration of sodium in the leaked fluid has displaced the other major cations on exchange sites of the natural sediment. The displaced cations effectively have been pushed ahead of the sodium-rich fluids. The displaced cations form a zone that almost reaches the depth of the contact between the upper and lower Plio- Pleistocene units at 47.5 meters (156 feet bgs).

Based on the water extract anion and major cation data, the leading edge of the leaked tank fluids appears to have affected the sediment at borehole 299-W23-19 from 22.2 meters (73 feet bgs) to near the base of the upper Plio-Pleistocene unit at 47.5 meters (156 feet bgs). The bulk of the leaked fluid currently resides in the upper Plio-Pleistocene unit between 38.1 and 47.5 meters (125 and 156 feet bgs). This is slightly deeper than the plume found under tank SX-108 (borehole C3082 [Serne et al. 2002e] and at tank SX-109 (borehole 41-09-39 [Serne et al. 2002b]), both of which have the major part of plumes in the shallower Hanford formation $\mathrm{H} 2$ unit.

Tables 4.8 and 4.9 give data for trace metal concentrations from both the dilution-corrected 1:1 sediment-to-water extract analysis and the actual porewater (from ultracentrifuge) analysis. Figure 4.5 shows the concentration versus depth for several trace metals. Table 4.8 and Figure 4.5 show elevated concentrations of chromium and technetium-99 in the vadose zone porewater. Elevated chromium concentrations start at 20.6 meters (67.5 feet bgs) and continue down to just below the lower Hanford formation $\mathrm{H} 2$ unit contact. The largest chromium concentration is $122,342 \mu \mathrm{g} / \mathrm{L}$ at 32.6 meters (107 feet bgs). This is an extremely high concentration compared to chromium in the Hanford formation in uncontaminated boreholes, which is generally near the quantitation limit (about $1 \mu \mathrm{g} / \mathrm{L}$ ). Chromium concentration decreases significantly at 38.4 meters (126 feet bgs), 1.21 meters ( 4 feet) below the contact between the Hanford formation $\mathrm{H} 2$ unit and the underlying upper Plio-Pliestocene unit. Chromium concentrations remain slightly above background throughout the lower part of the borehole.

Technetium-99 is found in the ultracentrifuge-derived porewater and in the water extracts from 22.2 meters ( 73 feet bgs) all the way to the bottom of the borehole. There is an order of magnitude decrease in technetium- 99 concentrations at 47.5 meters (156 feet bgs), which coincides with decreases in most major cations and with nitrate and chloride. As with the major cations, nitrate, and chloride, the bulk of the technetium-99 contamination is in the upper Plio-Pleistocene unit. The largest technetium-99 concentration is $3291 \mathrm{pCi} / \mathrm{mL}$ at 39.3 meters (129 feet bgs).

The uranium concentrations in the porewater for selected samples from borehole 299-W23-19 may be slightly elevated compared to concentrations in nearby uncontaminated boreholes. Uranium in borehole 299-W23-19 is elevated between 22.2 meters (73 feet bgs) and the bottom of the Hanford formation H2 unit at 37.1 meters (122 feet bgs) with the largest concentration of $62 \mu \mathrm{g} / \mathrm{L}$ at 26.2 meters ( 86 feet bgs). Uranium concentrations also are elevated in the lower Plio-Pleistocene unit where the highest 
concentrations of uranium in borehole 299-W23-19 are up to $217 \mu \mathrm{g} / \mathrm{L}$. Uranium concentrations in water extracts from two nearby uncontaminated boreholes are greater than $2 \mu \mathrm{g} / \mathrm{L}$ except in the lower PlioPleistocene unit where uranium concentrations up to $10 \mu \mathrm{g} / \mathrm{L}$ were noted (Serne et al. 2002a). Uranium in borehole 299-W23-19, and outside of the two zones mentioned above, is greater than $2 \mu \mathrm{g} / \mathrm{L}$ in all analyzed samples. Measured uranium concentrations in water extracts from the water extracts in borehole 299-W23-19 are only slightly higher than those from uncontaminated boreholes such that some or all of the values reported here may be false positives. The same can be said for the selenium distribution in the porewater that also seems slightly elevated above background between 22.2 meters (73 feet bgs) and the bottom of the Hanford formation H1 unit at 30.5 meters (100 feet bgs). Selenium in borehole 299-W23-19 reaches $34 \mu \mathrm{g} / \mathrm{L}$, whereas selenium in uncontaminated boreholes is generally less than the quantitation limit except in the lower Plio-Pleistocene unit where selenium up to $36 \mu \mathrm{g} / \mathrm{L}$ has been noted (Serne et al. 2002a).

Arsenic and molybdenum concentrations in the water extracts from borehole 299-W23-19 appear to show a small amount of contamination in the Hanford formation between 22.2 and 32.9 meters (73 and 108 feet bgs) where concentrations reach $3463 \mu \mathrm{g} / \mathrm{L}$ and $3495 \mu \mathrm{g} / \mathrm{L}$ respectively. Concentrations of arsenic in water extracts of uncontaminated boreholes is less than $40 \mu \mathrm{g} / \mathrm{L}$ and generally less than $30 \mu \mathrm{g} / \mathrm{L}$ (Serne et al. 2002a). Molybdenum concentrations in water extract of uncontaminated boreholes are less than 20 and generally less than $15 \mu \mathrm{g} / \mathrm{L}$ except in the Plio-Pleistocene where higher values are common.

The porewaters from well 299-W23-19 do not appear to be elevated in silver or cadmium and, although difficult to evaluate, lead.

Table 4.7 Cation Concentrations of Actual (from ultracentrifugation) and Dilution-corrected (from 1:1 Water Extracts) Porewaters

\begin{tabular}{|c|c|c|c|c|c|c|c|c|c|c|c|c|c|c|}
\hline $\begin{array}{c}\text { Sample } \\
\text { ID }\end{array}$ & $\begin{array}{c}\text { Depth } \\
(\mathrm{ft} \text { bgs) }\end{array}$ & Dil. Fac. & $\begin{array}{c}\text { water:soil } \\
\text { Ratio }\end{array}$ & $\begin{array}{c}\mathrm{Al} \\
(\mu \mathrm{g} / \mathrm{L}) \\
\end{array}$ & $\begin{array}{c}\mathrm{Ba} \\
(\mu \mathrm{g} / \mathrm{L}) \\
\end{array}$ & $\begin{array}{c}\mathrm{Ca} \\
(\mathrm{mg} / \mathrm{L}) \\
\end{array}$ & $\begin{array}{c}\mathrm{Fe} \\
(\mu \mathrm{g} / \mathrm{L})\end{array}$ & $\begin{array}{c}\mathrm{K} \\
(\mathrm{mg} / \mathrm{L} \\
\end{array}$ & $\begin{array}{r}\mathrm{Mg} \\
(\mathrm{mg} / \mathrm{L} \\
\end{array}$ & $\begin{array}{c}\mathrm{Na} \\
(\mathrm{mg} / \mathrm{L}) \\
\end{array}$ & $\begin{array}{c}\mathrm{Si} \\
\mathrm{mg} / \mathrm{L}) \\
\end{array}$ & $\begin{array}{c}\mathrm{Sr} \\
(\mu \mathrm{g} / \mathrm{L}) \\
\end{array}$ & $\begin{array}{r}\mathrm{SO} 4 \\
(\mathrm{mg} / \mathrm{L}) \\
\end{array}$ & $\begin{array}{r}\mathrm{Mn} \\
(\mu \mathrm{g} / \mathrm{L} \\
\end{array}$ \\
\hline $17 \mathrm{DE}$ & \multirow{3}{*}{64.75} & 505 & 1.00 & $<250$ & 65 & 62 & 71 & 19 & 8 & 164 & 94 & 315 & 157 & 2 \\
\hline 7DE-aft & & 10.70 & 0.96 & 164 & 161 & 72 & 308 & 27 & 14 & 316 & 198 & 123 & 126 & 12 \\
\hline 7DE-ufa & & 1.0 & & $<50$ & 74 & 52 & $(39)$ & 9 & 10 & 90 & 29 & (240) & 159 & 9) \\
\hline $19 \mathrm{D}$ & \multirow{2}{*}{.25} & 9.61 & 1.18 & 1554 & 154 & 12 & 3340 & 22 & 1 & 825 & 148 & 98 & 273 & 67 \\
\hline 19D-ufa & & 1.00 & & $(42)$ & 6 & 26.2 & (21) & 14 & 3 & 1348 & 0 & 268 & 6 & (12) \\
\hline $20 \mathrm{E}$ & \multirow{2}{*}{-79.75} & $1.1 \%$ & 1.00 & \begin{tabular}{|l|l|}
1100 \\
\end{tabular} & 156 & 9 & 9829 & 19 & 1 & 1472 & 125 & 74 & 214 & 140 \\
\hline 20E-ufa & & 1.00 & & $(51)$ & 65 & 20 & $(51)$ & 14.8 & 2.5 & 1177 & 15.8 & 197 & 233 & (5) \\
\hline 35IH-aft & 141.85 & 6.30 & 1.00 & $<500$ & 4275 & 2885 & 151 & 89 & 817 & 533 & 64 & 1704 & 73 & 30 \\
\hline 35IH-ufa & 141.85 & 1.00 & & $<50$ & 499 & 3476 & $(10$ & $\Delta c$ & 8 & 427 & 15 & 2971 & 34 & $(67)$ \\
\hline E-aft & 3.35 & & 1.04 & $<500$ & 75 & 2361 & 151 & 82 & 723 & $4 / 0$ & 14 & 1366 & 01 & (20 \\
\hline 35F-ufa & 3.35 & & & $<50$ & 6338 & 3140 & $(28)$ & 4 & 80 & 41 & 15 & 26136 & 34 & $(45$ \\
\hline 8A-aft & 5.10 & & 1.00 & $<500$ & 448 & 152 & 680 & 5 & 7 & 242 & 162 & 513 & 151 & 30 \\
\hline 8A-ufa & 55.1 & & & $<50$ & 31 & 72 & $(16$ & 1 & 65 & 106 & 23 & 884 & 1 & (3) \\
\hline
\end{tabular}


(a) multiply by 0.3048 to convert to meters

ufa $=$ the actual porewater obtained by ultracentrifugation

aft indicates 1:1 sediment-to-water extract AFTER ultracentrifugation to remove porewater

Extracts with incorrect water-to-sediment ratios are highlighted in orange.

Note that some analytes are reported as $\mu \mathrm{g} / \mathrm{L}$ and others $\mathrm{mg} / \mathrm{L}$ 
Table 4.8 Trace Metal Concentrations of Actual (from ultracentrifugation) and Dilution-corrected (from 1:1 Water Extracts) Porewaters in Sediment from Borehole 299-W23-19

\begin{tabular}{|c|c|c|c|c|c|c|c|c|c|c|c|c|}
\hline \multirow{2}{*}{ Sample ID } & \multirow{2}{*}{$\begin{array}{l}\text { Depth } \\
(\mathbf{f t} \text { bgs })^{\mathrm{a}}\end{array}$} & \multirow[b]{2}{*}{ Dil. Fac. } & \multirow{2}{*}{$\begin{array}{c}\text { Water:soil } \\
\text { Ratio* }\end{array}$} & \multirow{2}{*}{$\begin{array}{c}{ }^{99} \mathrm{Tc} \\
(\mathrm{pCi} / \mathrm{mL})\end{array}$} & $\mathbf{U}$ & $\mathrm{Cr}$ & As & Se & Mo & Ag & Cd & $\mathbf{P b}$ \\
\hline & & & & & \multicolumn{8}{|c|}{$(\mu \mathrm{g} / \mathrm{L})$} \\
\hline $09 \mathrm{E}$ & 32.95 & 9.85 & 1.00 & $<8$ & 11.7 & $<25$ & 244 & $<25$ & 87 & $<1$ & $<1.0$ & 7.4 \\
\hline $15 \mathrm{~A}$ & 54.50 & 17.13 & 1.00 & $<15$ & 19.4 & $<9$ & 131 & $<43$ & 149 & $<4$ & $<4.3$ & $<286$ \\
\hline $16 \mathrm{~A}$ & 57.45 & 15.82 & 1.00 & $<13$ & 15.7 & $<8$ & 162 & $<40$ & 191 & $<4$ & $<4.0$ & 39.6 \\
\hline $17 \mathrm{~J}$ & 61.75 & 18.84 & 1.00 & $<16$ & 20.2 & 9.6 & 248 & $<47$ & 93 & $<5$ & $<4.7$ & 244.8 \\
\hline $17 \mathrm{G}$ & 63.25 & 11.13 & 1.00 & $<9$ & 14.2 & $<6$ & 213 & $<28$ & 62 & $<3$ & $<2.8$ & $<28$ \\
\hline $17 \mathrm{~F}$ & 63.75 & 8.53 & 1.00 & $<7$ & 12.9 & 12.2 & 185 & $<21$ & 45 & $<2$ & $<2.1$ & $<21$ \\
\hline 17F-dup & 64.75 & 8.56 & 1.01 & $<7$ & 13.2 & $<4$ & 187 & $<21$ & 44 & $<2$ & $<2.1$ & $<21$ \\
\hline $17 \mathrm{DE}$ & 64.75 & 5.05 & 1.00 & $<2$ & 5.5 & 8.1 & 103 & 6.3 & 60 & $<1$ & $<0.2$ & 0.8 \\
\hline 17DE-aft & 64.75 & 10.70 & 0.96 & $<9$ & 12.4 & 5.3 & 327 & $<27$ & 91 & $<3$ & $<2.7$ & $<26.75$ \\
\hline $17 \mathrm{C}$ & 65.25 & 9.03 & 1.00 & $<8$ & 9.5 & $<4.5$ & 185 & $<23$ & 115 & $<2$ & $<36.1$ & $<23$ \\
\hline $18 \mathrm{I}$ & 67.45 & 5.74 & 1.00 & $<5$ & 5.6 & 96 & 135 & $<14$ & 49 & $<1$ & $<1.4$ & 19.7 \\
\hline $18 \mathrm{E}$ & 69.45 & 10.19 & 1.00 & $<9$ & 9.0 & 308 & 190 & $<25$ & 147 & $<1$ & $<1.0$ & 6.9 \\
\hline $18 \mathrm{C}$ & 70.45 & 6.56 & 1.00 & $<6$ & 15.6 & 3224 & 273 & $<16$ & 152 & 0.9 & $<0.7$ & 6.3 \\
\hline $18 \mathrm{~B}$ & 70.95 & 6.56 & 1.00 & $<6$ & 15.6 & 3343 & 268 & $<16$ & 154 & 1.0 & 3.9 & 8.4 \\
\hline $19 \mathrm{H}$ & 73.25 & 27.70 & 1.00 & 357.0 & 55.1 & 43262 & 1175 & 34 & 1352 & 7.5 & 2.2 & 15.2 \\
\hline $19 \mathrm{G}$ & 73.75 & 7.49 & 0.86 & 312.4 & 23.1 & 37442 & 001 & 34 & 1179 & 7.1 & 1.8 & 12.1 \\
\hline $19 \mathrm{E}$ & 74.75 & 7.09 & 1.00 & 333.9 & 23.6 & 48547 & 617 & 22 & 1028 & 6.1 & 1.0 & 8.1 \\
\hline 19D & 75.25 & 9.61 & 1.18 & 317.7 & 32.7 & 30391 & 2266 & 26 & 656 & 3.9 & 1.4 & 7.2 \\
\hline $20 \mathrm{~F}$ & 79.25 & 9.47 & 0.76 & 284.4 & 45.4 & 35668 & 2630 & 26 & 1412 & 4.0 & 2.0 & 7.1 \\
\hline 20F-dup & 79.25 & 9.38 & 1.04 & 245.8 & 41.2 & 29695 & 2345 & 18 & 1090 & 3.4 & 1.6 & 16.6 \\
\hline $20 \mathrm{E}$ & 79.75 & 7.79 & 1.00 & 198.2 & 20.7 & 21235 & 3463 & 22 & 667 & 4.1 & 1.4 & 34.8 \\
\hline $20 \mathrm{D}$ & 80.25 & 6.00 & 1.00 & 192.4 & 15.6 & 19834 & 2433 & 20 & 523 & 1.5 & 1.5 & 15.0 \\
\hline $21 \mathrm{~A}$ & 86.00 & 13.58 & 0.61 & 94.4 & 62.3 & 5422 & 2229 & 20 & 310 & 1.0 & 0.8 & 4.1 \\
\hline $22 \mathrm{D}$ & 88.10 & 6.07 & 1.00 & 288.9 & 51.6 & 39232 & 616 & 17 & 731 & 3.7 & 0.8 & 10.0 \\
\hline $24 \mathrm{~A}$ & 95.00 & 10.82 & 1.00 & 330.9 & 26.0 & 40792 & 1063 & 24 & 3850 & 4.4 & 2.7 & 17.0 \\
\hline $25 \mathrm{~B}$ & 99.06 & 14.17 & 0.81 & 120.2 & 19.7 & 27723 & 868 & 27 & 164 & 0.4 & 0.9 & 6.2 \\
\hline 25B-dup & 99.06 & 15.85 & 0.97 & 122.3 & 20.7 & 28227 & 1099 & 18 & 144 & 0.5 & 0.6 & 2.3 \\
\hline $25 \mathrm{~A}$ & 100.06 & 11.40 & 0.79 & 394.5 & 13.1 & 85566 & 466 & 45 & 273 & 2.3 & 0.8 & 9.6 \\
\hline $26 \mathrm{E}$ & 102.95 & 13.75 & 1.00 & 177.2 & 36.3 & 35900 & 1146 & 18 & 3495 & 1.0 & 4.3 & 9.1 \\
\hline $26 \mathrm{~A}$ & 104.95 & 12.21 & 1.00 & 155.3 & 33.2 & 16064 & 801 & 18 & 3288 & 0.0 & 3.7 & 3.9 \\
\hline $27 \mathrm{G}$ & 107.05 & 15.02 & 1.00 & 545.7 & 14.7 & 122342 & 291 & 38 & 1875 & 3.8 & 3.8 & 37.6 \\
\hline $29 \mathrm{D}$ & 116.35 & 13.67 & 1.00 & 506.2 & 14.1 & 93333 & 70 & 50 & 137 & 3.4 & 3.4 & 34.2 \\
\hline $30 \mathrm{G}$ & 123.85 & 11.13 & 1.00 & 919.4 & 23.8 & 81170 & 33 & 73 & 332 & 0.6 & 1.3 & 2.5 \\
\hline $30 \mathrm{D}$ & 125.35 & 6.78 & 0.79 & 1504.7 & 6.6 & 42511 & 34 & 123 & 129 & 2.0 & 0.9 & 2.2 \\
\hline
\end{tabular}




\begin{tabular}{|c|c|c|c|c|c|c|c|c|c|c|c|c|}
\hline & Depth & & & & $\mathbf{U}$ & $\mathrm{Cr}$ & As & Se & Mo & Ag & Cd & $\mathbf{P b}$ \\
\hline Sample ID & $(\mathbf{f t} \text { bgs })^{\mathrm{a}}$ & Dil. Fac. & Ratio* & $(\mathbf{p C i} / \mathbf{m L})$ & \multicolumn{8}{|c|}{$(\mu \mathrm{g} / \mathrm{L})$} \\
\hline $30 \mathrm{~B}$ & 126.35 & 6.03 & 1.00 & 2034.1 & 6.5 & 1788 & 7.1 & 139 & 108 & 4.0 & 1.5 & 28.2 \\
\hline 30B-dup & 126.35 & 6.03 & 1.00 & 2344.4 & 7.1 & 2584 & 6.5 & 144 & 101 & 4.2 & 1.5 & 15.1 \\
\hline $31 \mathrm{G}$ & 129.15 & 6.62 & 1.00 & 3291.0 & 9.1 & 18 & 4.6 & 102 & 81 & 2.0 & 1.1 & 4.1 \\
\hline $31 \mathrm{G}$ & 129.15 & 5.15 & 1.00 & 3033.6 & 3.3 & 31 & 20 & 73 & 57 & 1.1 & 0.7 & 1.9 \\
\hline $31 \mathrm{~A}$ & 132.15 & 6.36 & 1.00 & 2498.7 & 4.0 & 0.01 & 15 & 45 & 50 & 1.4 & 0.8 & 1.1 \\
\hline $31 \mathrm{~A}$ & 132.15 & 6.35 & 1.00 & 2457.2 & 4.2 & 6.4 & 15 & 43 & 49 & 1.3 & 0.7 & 3.4 \\
\hline $32 \mathrm{D}$ & 134.25 & 11.80 & 0.84 & 1169.5 & 6.7 & 4.7 & 33 & 40 & 134 & 0.0 & 0.5 & 2.0 \\
\hline $32 \mathrm{~A}$ & 135.80 & 5.79 & 1.00 & 2109.7 & 8.2 & $<14$ & 2.9 & 68 & 67 & 1.9 & 0.7 & $<3$ \\
\hline $33 \mathrm{G}$ & 137.65 & 6.43 & 1.00 & 2235.4 & 8.2 & $<16$ & $<3$ & 78 & 71 & 1.6 & 1.0 & $<3$ \\
\hline $33 \mathrm{~B}$ & 140.15 & 6.45 & 1.00 & 2117.2 & 10.3 & $<16$ & $<3$ & 79 & 104 & 2.3 & 0.9 & $<3$ \\
\hline $35 \mathrm{~K}$ & "141.05" & 8.72 & 1.00 & 2204.1 & 18.5 & $<22$ & 5.0 & 84 & 164 & 1.4 & 1.2 & $<4$ \\
\hline $35 \mathrm{IH}-\mathrm{aft}$ & 141.85 & 6.30 & 1.00 & 1772.9 & 9.4 & 6.6 & 5.4 & 72 & 91 & 1.6 & $<1.6$ & $<16$ \\
\hline $35 \mathrm{~F}-\mathrm{aft}$ & 143.35 & 7.18 & 1.04 & 1613.3 & 10.5 & 5.9 & 6.5 & 49 & 100 & $<2$ & $<1.8$ & $<18$ \\
\hline $35 \mathrm{C}$ & 144.85 & 6.15 & 1.00 & 1138.1 & 7.0 & $<15$ & 6.5 & 28 & 121 & $<1$ & $<0.6$ & $<3$ \\
\hline $35 \mathrm{~A}$ & 145.85 & 3.89 & 1.00 & 1115.8 & 6.7 & 3.7 & 4.1 & 29 & 48 & $<1$ & $<1.0$ & $<10$ \\
\hline $36 \mathrm{D}$ & 147.45 & 10.20 & 1.00 & 1170.7 & 7.4 & 3.6 & 18 & 23 & 141 & $<1$ & 0.6 & 3.4 \\
\hline $36 \mathrm{~B}$ & 148.45 & 4.35 & 1.00 & 831.2 & 8.4 & 6.5 & 5.8 & 22 & 74 & $<1$ & $<1.1$ & $<11$ \\
\hline 37D & 149.65 & 7.72 & 1.00 & 1438.3 & 25.3 & $<19$ & 4.0 & 50 & 208 & $<1$ & $<2.1$ & $<4$ \\
\hline $38 \mathrm{G}$ & 152.10 & 4.32 & 1.00 & 237.3 & 7.6 & 4.3 & 19 & 18 & 83 & $<0$ & 0.2 & 0.8 \\
\hline $38 \mathrm{G}$ & 152.10 & 4.32 & 1.00 & 249.6 & 7.7 & 3.8 & 20 & 18 & 88 & $<0$ & 0.2 & 0.6 \\
\hline $38 \mathrm{E}$ & 153.10 & 7.11 & 1.00 & 152.8 & 12.2 & 5.2 & 43 & 24 & 147 & $<2$ & $<1.8$ & $<18$ \\
\hline 38E-dup & 153.10 & 7.11 & 1.00 & 142.8 & 12.0 & 4.4 & 44 & 20 & 156 & $<2$ & $<1.8$ & $<18$ \\
\hline 38A-aft & 155.10 & 10.92 & 1.00 & 37.3 & 20.1 & $<5$ & 391 & $<27$ & 157 & $<3$ & $<2.7$ & $<27$ \\
\hline 39D & 155.35 & 9.49 & 0.82 & 293.2 & 36.1 & 9.9 & 28 & 21 & 309 & $<1$ & 0.8 & 12 \\
\hline $39 \mathrm{C}$ & 155.85 & 12.83 & 1.00 & 138.6 & 36.1 & $<32$ & 510 & $<32$ & 160 & $<1$ & $<1.3$ & $<6$ \\
\hline 39c-dup & 155.85 & 12.82 & 1.00 & 133.0 & 42.5 & $<32$ & 483 & $<32$ & 165 & $<1$ & $<1.3$ & $<6$ \\
\hline $40 \mathrm{~B}$ & 157.75 & 20.49 & 1.00 & 23.3 & 99.1 & 10.4 & 448 & $<51$ & 220 & $<5$ & $<5.1$ & 51.2 \\
\hline 40 & 158.65 & 10.96 & 1.38 & 29.4 & 217.4 & 11.2 & 194 & 32 & 445 & $<1$ & 0.9 & 2.9 \\
\hline 17SK & 159.75 & 11.92 & 1.00 & 30.7 & 25.3 & 64.6 & 276 & 30 & 886 & $<1$ & 1.3 & 1.4 \\
\hline 18SKA & 161 & 14.68 & 1.01 & 23.6 & 20.0 & 42.9 & 332 & 27 & 1276 & $<1$ & 2.1 & 8.4 \\
\hline $18 \mathrm{SKB}$ & 161.00 & 19.78 & 1.04 & 52.3 & 28.7 & 83.4 & 388 & 34 & 1417 & $<2$ & 2.5 & 2.4 \\
\hline $18 \mathrm{SKC}$ & 166.50 & 34.13 & 1.01 & 53.8 & 3.5 & 41.8 & 12 & 24 & 1157 & $<3$ & 2.1 & 6.3 \\
\hline 19SKA & 166.50 & 74.25 & 1.00 & 68.0 & 8.9 & 44.5 & 40 & 52 & 1547 & $<7$ & 3.9 & 9.4 \\
\hline 19SKB & 176.00 & 80.64 & 1.00 & 45.1 & 11.6 & 19.0 & 115 & 54 & 1524 & $<8$ & 4.0 & 26.1 \\
\hline $41 \mathrm{~A}$ & 184.45 & 25.37 & 1.00 & 13.1 & 9.5 & 14.1 & 94 & $<63$ & 384 & $<6$ & $<6.3$ & $<63$ \\
\hline 19SKC & 185 & 114.65 & 1.01 & 31.1 & 12.6 & $<115$ & 145 & 83 & 2368 & $<11$ & 4.6 & 8.7 \\
\hline
\end{tabular}




\begin{tabular}{|c|c|c|c|c|c|c|c|c|c|c|c|c|}
\hline \multirow[b]{2}{*}{ Sample II } & \multirow{2}{*}{$\begin{array}{l}\text { Depth } \\
(\text { ft bgs })^{\mathrm{a}}\end{array}$} & \multirow[b]{2}{*}{ Dil. Fac. } & \multirow{2}{*}{$\begin{array}{c}\text { Water:soil } \\
\text { Ratio* }\end{array}$} & \multirow{2}{*}{$\begin{array}{c}{ }^{99} \mathrm{Tc} \\
(\mathrm{pCi} / \mathrm{mL})\end{array}$} & $\mathbf{U}$ & $\mathrm{Cr}$ & As & $\mathrm{Se}$ & Mo & Ag & Cd & $\mathbf{P b}$ \\
\hline & & & & & \multicolumn{8}{|c|}{$(\mu \mathrm{g} / \mathrm{L})$} \\
\hline 20SKA & 185 & 78.06 & 1.00 & 14.6 & 19.8 & 0.0 & 191 & 47 & 1212 & $<8$ & 4.2 & 33.7 \\
\hline 20SKB & 189.5 & 82.45 & 0.94 & 25.2 & 12.2 & 12.9 & 205 & 89 & 1887 & $<8$ & 4.0 & 13.9 \\
\hline 42 & 194.55 & 17.10 & 1.00 & 5.6 & 34.2 & 9.2 & 50 & 42 & 279 & $<4$ & $<4.3$ & $<43$ \\
\hline $43 \mathrm{~B}$ & 204.50 & 16.25 & 1.00 & 51.1 & 25.0 & $<8$ & 23 & $<41$ & 247 & $<4$ & $<4.1$ & 47.4 \\
\hline $43 \mathrm{~A}$ & 205.15 & 41.39 & 1.00 & 176.2 & 9.5 & 23.7 & 217 & 25 & 576 & 0.2 & 2.2 & 6.9 \\
\hline
\end{tabular}




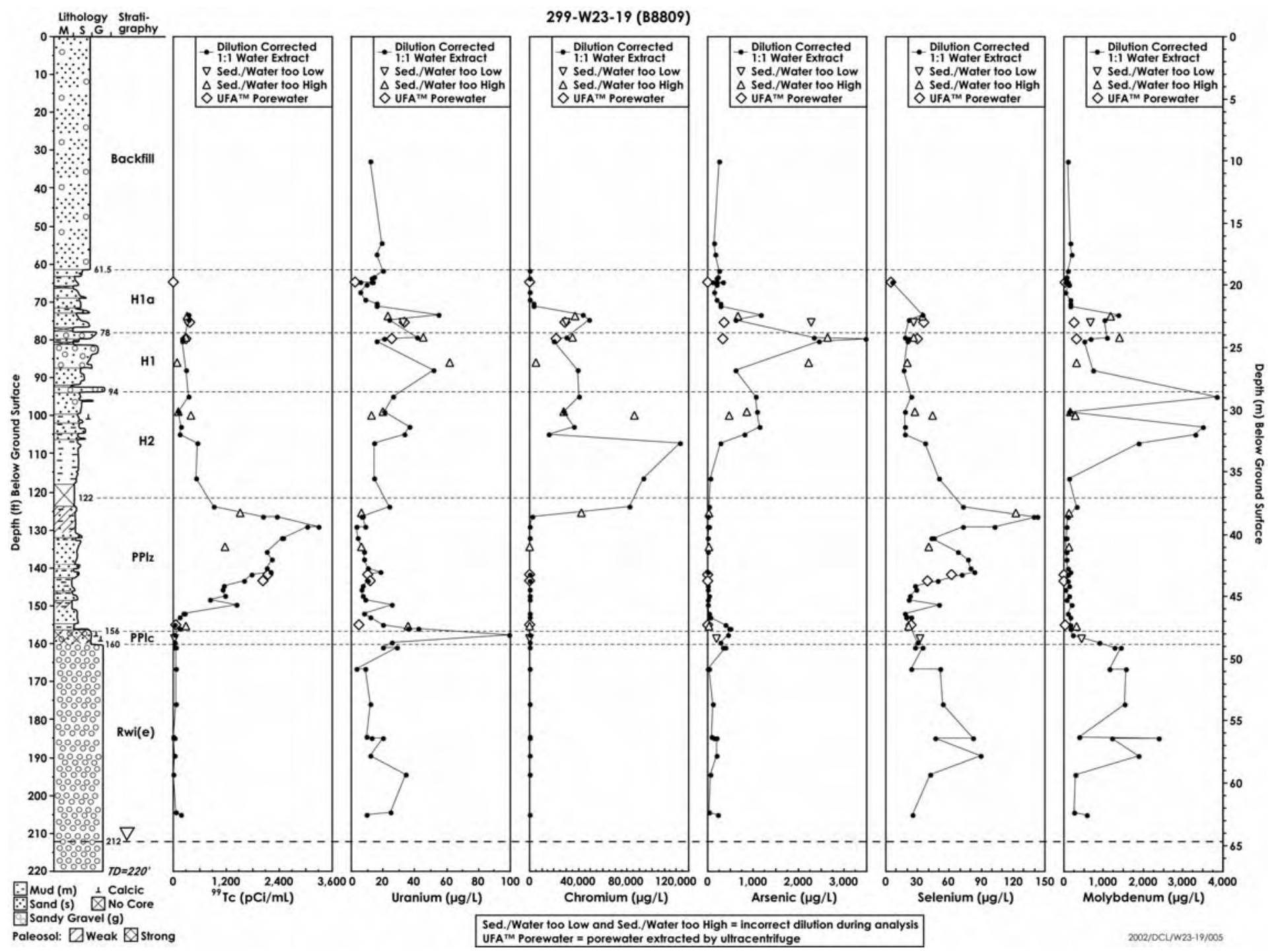

Figure 4.5 Trace Metals Concentrations versus Depth in Dilution-corrected [from sediment:water extracts] and Actual (from ultracentrifuge) Porewaters from Borehole 299-W23-19 Sediment. 
If dilution was the only reason for a decrease in concentrations of mobile constituents with depth, then the ratios of one constituent to another should remain constant. Table 4.10 gives the ratios of the water extract of various constituents to nitrate or to technetium-99 from sediment in well 299-W23-19.

The data in Table 4.10 show that the ratios technetium-99-to-nitrate, chloride-to-nitrate, and chlorideto-technetium-99 are fairly constant throughout the borehole suggesting that these three constituents migrate through the vadose zone together and do not interact with the sediment (in reality the constant ratios could also mean that they have the same $\mathrm{K}_{\mathrm{d}}$ or sorption tendencies with the sediment). The other ratios show some variation with depth suggesting that those metals and trace metals undergo some reaction with the sediment. As the ratio of a given constituent with nitrate or technetium- 99 decreases, the reaction of that constituent with the sediment becomes stronger and if is removed from the percolating fluids. Ratios that show significant retardation are shaded in Table 4.10 such that the reader can see the depths at which the various constituents are removed from the soil water. In general, the reactive constituents arsenic, chromium, molybdenum, selenium, and sodium have sorbed onto the vadose zone sediment at the base of the Hanford formation $\mathrm{H} 2$ unit or within the upper Plio-Pliestocene unit.

The ratio of technetium-99 to nitrate is fairly constant throughout the borehole at about $100 \mathrm{pCi} / \mathrm{mg}$, which is similar to the ratio in the groundwater at the bottom of the borehole. This technetium-99-tonitrate ratio is also similar to that predicted in the tank supernates at the time of the SX-115 leak. Despite the similarity in the technetium-99 to nitrate ratios from both the vadose zone porewater and the groundwater, contamination is much less in the vadose zone than in the groundwater. Therefore, the pathway of contaminants from tank SX-115 through the lower Plio-Pleistocene unit and the Ringold Formation to groundwater must be near but up hydraulic gradient from well 299-W23-19.

A detailed look at the chemical compositions of the actual porewaters with the dilution-corrected porewaters (see Tables 4.5, 4.7, and 4.9) shows that the actual porewaters are consistently lower in aluminum, iron, manganese, silicon, and sodium than are the dilution-corrected porewaters. This suggests that the 1:1 water extract causes dissolution of small amounts of these fairly insoluble (except sodium), major, mineral-forming elements. The sodium data may reflect the dissolution of small amounts of sodium nitrate that precipitated from the leaked fluids in the sediment although the sodium and nitrate concentrations are well below the solubility limit for sodium nitrate. The actual porewaters also contain lower concentrations of the trace metals arsenic, chromium (except sediment with high chromium (VI) contamination), lead, and selenium and slightly larger concentrations of technetium-99 than do the dilution-corrected water extracts. 
Table 4.9 Trace Metal Concentrations from Actual (ultracentrifuge) and Dilution-corrected (water extracted) Porewaters

\begin{tabular}{|c|c|c|c|c|c|c|c|c|c|c|c|c|}
\hline & Depth & & water:soil & & $\mathbf{U}$ & $\mathrm{Cr}$ & As & Se & Mo & Ag & Cd & $\mathbf{P b}$ \\
\hline \begin{tabular}{|c|} 
Sample \\
ID
\end{tabular} & (ft bgs) & Dil. Fac. & & $(\mathbf{p C i} / \mathbf{m L})$ & \multicolumn{8}{|c|}{$(\mu \mathrm{g} / \mathrm{L})$} \\
\hline $17 \mathrm{DE}$ & \multirow{3}{*}{64.75} & 5.05 & 1.00 & $<2$ & 5.5 & 8.1 & 103 & 6.3 & 60 & $<1$ & $<0.2$ & 0.8 \\
\hline 17DE-aft & & 10.70 & 0.96 & $<9$ & 12.4 & 5.3 & 327 & $<27$ & 91 & $<3$ & $<2.7$ & $<26.75$ \\
\hline 17DE-ufa & & 1.00 & & 2.11 & 2.24 & 3.34 & 14.9 & 4.60 & 45.3 & $<0.25$ & $<0.25$ & $<2.5$ \\
\hline 19D & \multirow{2}{*}{75.25} & 9.61 & 1.18 & 317.7 & 32.7 & 30391 & 2266 & 26 & 656 & 3.9 & 1.4 & 7.2 \\
\hline 19D-ufa & & 1.00 & & 359.6 & 33.3 & 28894 & 362 & $(35)$ & 257 & $(1.2)$ & $(0.5)$ & $(1.4)$ \\
\hline $20 \mathrm{E}$ & \multirow{2}{*}{79.75} & 7.79 & 1.00 & 198.2 & 20.7 & 21235 & 3463 & 22 & 667 & 4.1 & 1.4 & 34.8 \\
\hline 20E-ufa & & 1.00 & & 296.8 & 25.7 & 22145 & 334 & (30) & 319 & $(0.7)$ & $(0.7)$ & $(1.2)$ \\
\hline 35IH-aft & 141.85 & 6.30 & 1.00 & 1773 & 9.4 & 6.6 & 5.4 & 72 & 91 & 1.6 & $<1.6$ & $<16$ \\
\hline $35 \mathrm{IH}-\mathrm{ufa}$ & 141.85 & 1.00 & & 2148 & 10.2 & 0.64 & 1.38 & 62.1 & 8.72 & 1.68 & 1.50 & $<2.5$ \\
\hline $35 \mathrm{~F}-\mathrm{aft}$ & 143.35 & 7.18 & 1.04 & 1613 & 10.5 & 5.9 & 6.5 & 49 & 100 & $<2$ & $<1.8$ & $<18$ \\
\hline 35F-ufa & 143.35 & 1.00 & & 2022 & 11.9 & $<0.5$ & 1.33 & 39.3 & 6.29 & $<1.09$ & 1.02 & 2.82 \\
\hline 38A-aft & 155.10 & 10.92 & 1.00 & 37.3 & 20.1 & $<5$ & 391 & $<27$ & 157 & $<3$ & $<2.7$ & $<27$ \\
\hline 38A-ufa & 155.1 & 1.00 & & 63 & 4.60 & 0.60 & 13.2 & 23.6 & 30.3 & $<0.25$ & 0.39 & $<2.5$ \\
\hline
\end{tabular}

Table 4.10 Ratio of Some Major and Trace Metals to the Mobile Species Nitrate and Technetium-99

\begin{tabular}{|c|c|c|c|c|c|c|c|c|c|c|c|c|c|}
\hline $\begin{array}{c}\text { Sample } \\
\text { ID }\end{array}$ & $\begin{array}{c}\text { Depth } \\
\text { (ft bgs) }\end{array}$ & $\begin{array}{l}{ }^{99} \mathrm{Tc} / \\
\mathrm{NO}_{3}\end{array}$ & $\begin{array}{c}\mathrm{Cr} / \\
\mathrm{NO}_{3}\end{array}$ & $\begin{array}{l}\mathrm{Mo} / \\
\mathrm{NO}_{3} \\
\end{array}$ & $\begin{array}{l}\mathrm{Na} / \\
\mathrm{NO}_{3} \\
\end{array}$ & $\begin{array}{c}\mathrm{Cl} / \\
\mathrm{NO}_{3} \\
\end{array}$ & $\begin{array}{l}\mathrm{U} / \\
{ }^{99} \mathrm{Tc} \\
\end{array}$ & $\begin{array}{c}\mathrm{Cr} / \\
{ }^{99} \mathrm{Tc}\end{array}$ & $\begin{array}{l}\mathrm{As} / \\
{ }^{99} \mathrm{Tc}\end{array}$ & $\begin{array}{c}\mathrm{Se} / \\
{ }^{99} \mathrm{Tc}\end{array}$ & $\begin{array}{l}\text { Mo/ } \\
{ }^{99} \mathrm{Tc}\end{array}$ & $\begin{array}{l}\mathrm{Na} / \\
{ }^{99} \mathrm{Tc}\end{array}$ & $\begin{array}{l}\mathrm{Cl} / \\
{ }^{99} \mathrm{Tc}\end{array}$ \\
\hline & & \multicolumn{12}{|c|}{ Hanford Formation H1a Unit } \\
\hline $19 \mathrm{H}$ & 73.25 & 0.09 & 11.42 & 0.36 & 0.81 & 0.01 & 0.15 & 121.2 & 3.29 & 0.10 & 3.79 & 8.63 & 0.16 \\
\hline $19 \mathrm{G}$ & 73.75 & 0.10 & 12.47 & 0.39 & 0.54 & 0.02 & 0.07 & 119.8 & 2.18 & 0.11 & 3.78 & 5.22 & 0.16 \\
\hline $19 \mathrm{E}$ & 74.75 & 0.11 & 15.89 & 0.34 & 0.50 & 0.02 & 0.07 & 145.4 & 1.85 & 0.07 & 3.08 & 4.62 & 0.14 \\
\hline \multirow[t]{2}{*}{ 19D } & 75.25 & 0.10 & 9.84 & 0.21 & 0.59 & 0.01 & 0.10 & 95.7 & 7.13 & 0.08 & 2.06 & 5.75 & 0.14 \\
\hline & & \multicolumn{12}{|c|}{ Hanford Formation H1 Unit } \\
\hline $20 \mathrm{~F}$ & 79.25 & 0.10 & 12.89 & 0.51 & 0.71 & 0.01 & 0.16 & 125.4 & 9.25 & 0.09 & 4.96 & 6.95 & 0.13 \\
\hline 20Fdup & 79.25 & 0.10 & 12.63 & 0.46 & 0.78 & 0.01 & 0.17 & 120.8 & 9.54 & 0.07 & 4.44 & 7.49 & 0.13 \\
\hline $20 \mathrm{E}$ & 79.75 & 0.09 & 9.87 & 0.31 & 0.68 & 0.01 & 0.10 & 107.1 & 17.47 & 0.11 & 3.36 & 7.42 & 0.13 \\
\hline $20 \mathrm{D}$ & 80.25 & 0.09 & 9.22 & 0.24 & 0.63 & 0.01 & 0.08 & 103.1 & 12.64 & 0.10 & 2.72 & 7.09 & 0.14 \\
\hline $21 \mathrm{~A}$ & 86.00 & 0.09 & 5.43 & 0.31 & 2.05 & 0.02 & 0.66 & 57.4 & 23.60 & 0.22 & 3.28 & 21.66 & 0.17 \\
\hline $22 \mathrm{D}$ & 88.10 & 0.11 & 14.45 & 0.27 & 0.54 & 0.01 & 0.18 & 135.8 & 2.13 & 0.06 & 2.53 & 5.11 & 0.14 \\
\hline
\end{tabular}




\begin{tabular}{|c|c|c|c|c|c|c|c|c|c|c|c|c|c|}
\hline $\begin{array}{c}\text { Sample } \\
\text { ID }\end{array}$ & \begin{tabular}{|c|}
$\begin{array}{c}\text { Depth } \\
(\mathbf{f t} \text { bgs })^{2}\end{array}$ \\
\end{tabular} & $\begin{array}{l}{ }^{99} \mathrm{Tc} / \\
\mathrm{NO}_{3} \\
\end{array}$ & $\begin{array}{l}\mathrm{Cr} / \\
\mathrm{NO}_{3} \\
\end{array}$ & $\begin{array}{l}\mathrm{Mo} / \\
\mathrm{NO}_{3}\end{array}$ & $\begin{array}{l}\mathrm{Na} / \\
\mathrm{NO}_{3} \\
\end{array}$ & $\begin{array}{c}\mathrm{Cl} / \\
\mathrm{NO}_{3}\end{array}$ & $\begin{array}{c}\mathrm{U} / \\
{ }^{99} \mathrm{Tc}\end{array}$ & $\begin{array}{l}\mathrm{Cr} / \\
{ }^{99} \mathrm{Tc}\end{array}$ & $\begin{array}{c}\mathrm{As} / \\
{ }^{99} \mathrm{Tc}\end{array}$ & $\begin{array}{c}\mathrm{Se} / \\
{ }^{99} \mathrm{Tc}\end{array}$ & $\begin{array}{l}\mathrm{Mo} / \\
{ }^{99} \mathrm{Tc}\end{array}$ & $\begin{array}{l}\mathrm{Na} / \\
{ }^{99} \mathrm{Tc}\end{array}$ & $\begin{array}{c}\mathrm{Cl} / \\
{ }^{99} \mathrm{Tc}\end{array}$ \\
\hline $24 \mathrm{~A}$ & 95.00 & 0.09 & 11.08 & 1.05 & 0.52 & 0.01 & 0.08 & 123.3 & 3.21 & 0.07 & 11.64 & 5.81 & 0.13 \\
\hline $25 \mathrm{~B}$ & 99.06 & 0.05 & 10.65 & 0.06 & 0.68 & 0.02 & 0.16 & 230.7 & 7.22 & 0.23 & 1.37 & 14.82 & 0.40 \\
\hline 25B-dup & 99.06 & 0.05 & 12.57 & 0.06 & 0.72 & 0.02 & 0.17 & 230.8 & 8.98 & 0.14 & 1.18 & 13.18 & 0.32 \\
\hline $25 \mathrm{~A}$ & 100.06 & 0.08 & 17.71 & 0.06 & 0.49 & 0.02 & 0.03 & 216.9 & 1.18 & 0.11 & 0.69 & 5.96 & 0.20 \\
\hline & & \multicolumn{12}{|c|}{ Hanford Formation H2 Unit } \\
\hline $26 \mathrm{E}$ & 102.95 & 0.08 & 16.64 & 1.62 & 0.74 & 0.02 & 0.20 & 202.6 & 6.47 & 0.10 & 19.72 & 8.96 & 0.25 \\
\hline $26 \mathrm{~A}$ & 104.95 & 0.17 & 17.47 & 3.58 & 1.48 & 0.03 & 0.21 & 103.5 & 5.16 & 0.11 & 21.18 & 8.76 & 0.20 \\
\hline $27 \mathrm{G}$ & 107.05 & 0.11 & 23.78 & 0.36 & 0.48 & 0.01 & 0.03 & 224.2 & 0.53 & 0.07 & 3.44 & 4.50 & 0.13 \\
\hline 29D & 116.35 & 0.08 & 14.60 & 0.02 & 0.25 & 0.01 & 0.03 & 184.4 & 0.14 & 0.10 & 0.27 & 3.20 & 0.16 \\
\hline $30 \mathrm{G}$ & 123.85 & 0.10 & 8.44 & 0.03 & 0.15 & 0.01 & 0.03 & 88.3 & 0.04 & 0.08 & 0.36 & 1.61 & 0.12 \\
\hline & & \multicolumn{12}{|c|}{ Upper Plio-Pleistocene Unit } \\
\hline $30 \mathrm{D}$ & 125.35 & 0.10 & 2.88 & 0.01 & 0.08 & 0.01 & & 28.3 & 0.02 & 0.08 & 0.09 & 0.74 & 0.08 \\
\hline $30 \mathrm{~B}$ & 126.35 & 0.11 & 0.09 & 0.01 & 0.07 & 0.01 & & 0.9 & & 0.07 & 0.05 & 0.63 & 0.08 \\
\hline 30B-dup & 126.35 & 0.11 & 0.12 & 0.00 & 0.07 & 0.01 & & 1.1 & & 0.06 & 0.04 & 0.60 & 0.07 \\
\hline $31 \mathrm{G}$ & 129.15 & 0.13 & & 0.00 & 0.03 & 0.01 & & 0.01 & & 0.03 & 0.02 & 0.25 & 0.07 \\
\hline $31 \mathrm{G}$ & 129.15 & & & & & & & 0.01 & 0.01 & 0.02 & 0.02 & 0.22 & 0.00 \\
\hline $31 \mathrm{~A}$ & 132.15 & 0.12 & & 0.00 & 0.03 & 0.01 & & & 0.01 & 0.02 & 0.02 & 0.26 & 0.08 \\
\hline $31 \mathrm{~A}$ & 132.15 & & & & & & & & 0.01 & 0.02 & 0.02 & 0.24 & \\
\hline $32 \mathrm{D}$ & 134.25 & 0.12 & & 0.01 & 0.05 & 0.01 & 0.01 & & 0.03 & 0.03 & 0.11 & 0.44 & 0.10 \\
\hline $32 \mathrm{~A}$ & 135.80 & 0.13 & & 0.00 & 0.03 & 0.01 & & 0.01 & & 0.03 & 0.03 & 0.25 & 0.07 \\
\hline $33 \mathrm{G}$ & 137.65 & 0.13 & & 0.00 & 0.03 & 0.01 & & 0.01 & & 0.03 & 0.03 & 0.23 & 0.08 \\
\hline $33 \mathrm{~B}$ & 140.15 & 0.12 & & 0.01 & 0.03 & 0.01 & & 0.01 & & 0.04 & 0.05 & 0.25 & 0.08 \\
\hline $35 \mathrm{~K}$ & 141.05 & 0.10 & & 0.01 & 0.03 & 0.01 & 0.01 & 0.01 & & 0.04 & 0.07 & 0.31 & 0.09 \\
\hline $35 \mathrm{IH}-$ & 141.85 & & & & & & 0.01 & & & 0.04 & 0.05 & 0.30 & \\
\hline 35F-aft & 143.35 & & & & & & 0.01 & & & 0.03 & 0.06 & 0.30 & \\
\hline $35 \mathrm{C}$ & 144.85 & 0.12 & & 0.01 & 0.04 & 0.01 & 0.01 & & 0.01 & 0.02 & 0.11 & 0.34 & 0.09 \\
\hline $35 \mathrm{~A}$ & 145.85 & 0.12 & & 0.01 & 0.04 & 0.01 & 0.01 & & & 0.03 & 0.04 & 0.37 & 0.09 \\
\hline $36 \mathrm{D}$ & 147.45 & 0.15 & & 0.02 & 0.06 & 0.01 & 0.01 & & 0.02 & 0.02 & 0.12 & 0.37 & 0.09 \\
\hline $36 \mathrm{~B}$ & 148.45 & 0.14 & & 0.01 & 0.06 & 0.01 & 0.01 & 0.01 & 0.01 & 0.03 & 0.09 & 0.41 & 0.09 \\
\hline $37 \mathrm{D}$ & 149.65 & 0.09 & & 0.01 & 0.03 & 0.01 & 0.02 & 0.00 & & 0.03 & 0.14 & 0.39 & 0.11 \\
\hline $38 \mathrm{G}$ & 152.10 & 0.13 & & 0.05 & 0.11 & 0.03 & 0.03 & 0.02 & 0.08 & 0.07 & 0.35 & 0.81 & 0.21 \\
\hline $38 \mathrm{G}$ & 152.10 & & & & & & 0.03 & 0.02 & 0.08 & 0.07 & 0.35 & 0.70 & \\
\hline $38 \mathrm{E}$ & 153.10 & 0.09 & & 0.09 & 0.15 & 0.03 & 0.08 & 0.03 & 0.28 & 0.16 & 0.96 & 1.68 & 0.34 \\
\hline 38E-dup & 153.10 & 0.09 & & 0.10 & 0.15 & 0.03 & 0.08 & 0.03 & 0.31 & 0.14 & 1.10 & 1.66 & 0.34 \\
\hline 38A-aft & 155.10 & & & & & & 0.54 & & 10.47 & 0.00 & 4.20 & 6.49 & \\
\hline 39D & 155.35 & 0.11 & & 0.11 & 0.13 & 0.03 & 0.12 & 0.03 & 0.10 & 0.07 & 1.05 & 1.20 & 0.25 \\
\hline
\end{tabular}




\begin{tabular}{|c|c|c|c|c|c|c|c|c|c|c|c|c|c|}
\hline $\begin{array}{c}\text { Sample } \\
\text { ID }\end{array}$ & $\begin{array}{c}\text { Depth } \\
(\mathrm{ft} \text { bgs })^{2}\end{array}$ & $\begin{array}{l}{ }^{99} \mathrm{Tc} / \\
\mathrm{NO}_{3} \\
\end{array}$ & $\begin{array}{c}\mathrm{Cr} / \\
\mathrm{NO}_{3} \\
\end{array}$ & $\begin{array}{l}\mathrm{Mo} / \\
\mathrm{NO}_{3} \\
\end{array}$ & $\begin{array}{l}\mathrm{Na} / \\
\mathrm{NO}_{3} \\
\end{array}$ & $\begin{array}{c}\mathrm{Cl} / \\
\mathrm{NO}_{3} \\
\end{array}$ & $\begin{array}{l}\mathrm{U} / \\
{ }^{99} \mathrm{Tc} \\
\end{array}$ & $\begin{array}{l}\mathrm{Cr} / \\
{ }^{99} \mathrm{Tc}\end{array}$ & $\begin{array}{l}\text { As/ } \\
{ }^{99} \mathrm{Tc}\end{array}$ & $\begin{array}{c}\mathrm{Se} / \\
{ }^{99} \mathrm{Tc}\end{array}$ & $\begin{array}{l}\text { Mo/ } \\
{ }^{99} \mathrm{Tc}\end{array}$ & $\begin{array}{l}\mathrm{Na} / \\
{ }^{99} \mathrm{Tc}\end{array}$ & $\begin{array}{l}\mathrm{Cl} / \\
{ }^{99} \mathrm{Tc} \\
\end{array}$ \\
\hline $39 \mathrm{C}$ & 155.85 & 0.12 & & 0.14 & 0.28 & 0.04 & 0.26 & & 3.68 & 0.00 & 1.15 & 2.30 & 0.35 \\
\hline 39c-dup & 155.85 & 0.12 & & 0.15 & 0.29 & 0.04 & 0.32 & & 3.63 & 0.00 & 1.24 & 2.33 & 0.35 \\
\hline
\end{tabular}

1 The ratio of technetium to nitrate has units $\mathrm{pCi} / \mu \mathrm{g}$; ratios of trace metals to nitrate have units $\mu \mathrm{g} / \mathrm{mg}$; the ratio of chloride to nitrate is dimensionless; the ratios of trace metals to technetium have units $\mathrm{ng} / \mathrm{pCi}$; the ratio of chloride to technetium has units of $\mu \mathrm{g} / \mathrm{pCi}$. Ratios sharing significant retardation are shaded orange.

2 multiply by 0.3048 to convert to meters

\subsubsection{Radionuclide Content in Vadose Zone Sediment}

The radioanalytical analyses performed on the sediment from borehole 299-W23-19 included direct gamma energy analysis, and analyses for tritium, strontium-90, and technetium-99. Unlike the sediment cores from borehole 41-09-39 and the slant borehole at tank SX-108 that were both very radioactive, the sediment from borehole 299-W23-19 showed no radioactivity during breakdown of the split-spoon sampler when recovering the samples.

The radionuclide content of the sediment is shown in Tables 4.11 to 4.13. The depth distribution of both potassium-40 and cesium-137 are shown in Table 4.11. The data show very low concentrations of cesium-137 between 0.1 and $0.2 \mathrm{pCi} / \mathrm{g}$, from 20.5 and 22.6 meters (67.45 to 74.25 feet bgs) in largevolume samples that were counted for up to 16 hours. The initial screening measurements made on small, 5-gram samples, which were counted for 10 to 60 minutes, also suggested small amounts of cesium between 21 and 26.8 meters (69 and 88 feet bgs) though the results are less precise than those from large samples. In all, very little cesium-137 was found despite the fact that borehole 299-W23-19 is only 3 meters ( 10 feet) from the south-south-west edge of tank SX-115 and that $\sim 17,000$ curies of cesium-137 are predicted to still exist in the vadose zone. The data suggest that cesium-137 is not very mobile at the SX-115 leak. Natural-occurring potassium-40 is the only other gamma-emitting radionuclide that was found.

The strontium-90 and tritium concentrations in the vadose zone sediment are shown in Table 4.12. The data give no indication of strontium-90 in the vadose zone sediment in this borehole, but there is a small amount of tritium detected between 21.3 meters ( 70 feet) and at least 38.1 meters ( 125 feet bgs). The tritium profile was not investigated in detail so it is difficult to state how deep in the vadose zone profile elevated levels could be measured. The largest tritium activity is at the contact between the Hanford formation $\mathrm{H} 2$ unit and the upper Plio-Pleistocene unit.

Table 4.13 shows the amounts of technetium-99 and uranium that were leached from the samples with $8 \mathrm{M}$ nitric acid. The amounts of technetium-99 and uranium that are water-leachable versus the amounts that are acid-leachable are shown in Figures 4.6 and 4.7, respectively. The figures show that the amount of water-leachable technetium-99 is essentially the same as that which is acid-leachable. As discussed earlier, most of the water-leachable technetium-99 is in the upper Plio-Pleistocene unit. The same appears to be true for the acid-leached technetium-99. 
The uranium content of the sediment does not appear to be elevated above values found in nearby uncontaminated boreholes (see Serne et al. 2002a). It is apparent from Figure 4.7 that very little acidleachable uranium is also water leachable. As discussed in Serne et al. 2002a, only about 10 to $20 \%$ of the total uranium in uncontaminated sediment is acid leachable, so the uranium concentrations shown in Table 4.13 are not representative of the total uranium content of the sediment.

\subsubsection{Total Carbon, Calcium Carbonate, and Organic Carbon in Vadose Zone Sediment from Borehole 299-W23-19}

The total carbon, inorganic carbon (calcium carbonate), and organic carbon contents of the vadose zone sediment at selected depths within borehole 299-W23-19 are shown on Table 4.14. The sediment in the backfill shows very uniform carbon distributions as is expected for well-mixed backfill material. The calcium carbonate content of the Hanford formation H1a unit ranges from 1 to $2.6 \mathrm{wt} \%$. The coarser grained Hanford formation $\mathrm{H} 1$ unit ranges from 1.7 to $1.85 \mathrm{wt} \%$ calcium carbonate and the deeper finer grained Hanford formation $\mathrm{H} 2$ unit contains from 1.9 to $2.73 \mathrm{wt} \%$ calcium carbonate. Thus, the calcium carbonate content is fairly uniform throughout the entire Hanford formation in borehole 299-W23-19. Somewhat higher calcium carbonate contents are found in the upper and lower Plio-Pleistocene units. One caliche sample from the lower Plio-Pleistocene contained $25 \mathrm{wt} \%$ calcium carbonate. The Ringold formation samples contained very little calcium carbonate.

Most calium carbonate in the Hanford Site vadose zone is a secondary precipitate forming as a result of pedogenic and weathering processes. Thus, as the calcium carbonate content increases, the porosity decreases. In most, but not all of the Hanford Site sediments, the decrease in porosity is very small. However, in the Plio-Pliestocene units the decrease can be significant such that the movement of fluids through the vadose zone are impacted.

None of the sediment showed elevated levels of organic carbon, but the upper Plio-Pleistocene unit contained slightly more organic carbon than did the younger Hanford formation sediment and older Ringold Formation sediment.

Table 4.11 Gamma Energy Analysis of Vadose Zone Sediment ${ }^{a}$

\begin{tabular}{|c|c|c|c|c|c|}
\hline \multirow[b]{2}{*}{ Sample ID } & \multirow{2}{*}{$\begin{array}{c}\text { Depth } \\
(\mathbf{f t} \text { bgs })^{\mathrm{a}}\end{array}$} & ${ }^{40} \mathrm{~K}$ & \pm uncertainty & ${ }^{137} \mathrm{Cs}$ & \pm uncertainty \\
\hline & & \multicolumn{2}{|c|}{$(\mathrm{pCi} / \mathrm{g})$} & \multicolumn{2}{|c|}{$(\mathrm{pCi} / \mathrm{g})$} \\
\hline \multicolumn{6}{|c|}{ Backfill } \\
\hline 01D & 7.55 & 12.72 & 0.41 & 0.065 & 0.015 \\
\hline $03 \mathrm{~J}$ & 11.85 & 16.12 & 0.75 & $<0.03$ & \\
\hline 03I & 12.35 & 27.27 & 1.06 & 0.04 & 0.03 \\
\hline $03 \mathrm{H}$ & 12.85 & 14.46 & 0.49 & $<0.03$ & \\
\hline $03 \mathrm{~A}$ & 16.35 & 13.58 & 0.49 & $<0.03$ & \\
\hline $04 \mathrm{~A}$ & 20.65 & 13.34 & 0.45 & $<0.03$ & \\
\hline 08B & 28.65 & 16.31 & 0.86 & $<0.03$ & \\
\hline
\end{tabular}




\begin{tabular}{|c|c|c|c|c|c|}
\hline \multirow[b]{2}{*}{ Sample ID } & \multirow{2}{*}{$\begin{array}{c}\text { Depth } \\
(\mathbf{f t} \text { bgs })^{\mathrm{a}}\end{array}$} & ${ }^{40} \mathbf{K}$ & \pm uncertainty & ${ }^{137} \mathrm{Cs}$ & \pm uncertainty \\
\hline & & \multicolumn{2}{|c|}{$(\mathrm{pCi} / \mathrm{g})$} & \multicolumn{2}{|c|}{$(\mathrm{pCi} / \mathrm{g})$} \\
\hline 09E & 32.95 & 14.37 & 0.27 & 0.051 & 0.009 \\
\hline $09 \mathrm{E}$ & 32.95 & 15.71 & 1.37 & 0.314 & 0.121 \\
\hline $11 \mathrm{C}$ & 42.35 & 26.77 & 1.12 & $<0.03$ & \\
\hline $13 \mathrm{C}$ & 45.85 & 13.87 & 0.55 & $<0.03$ & \\
\hline $13 \mathrm{~A}$ & 46.85 & 16.88 & 0.9 & $<0.03$ & \\
\hline 13A-dup & 46.85 & 14.04 & 0.53 & $<0.03$ & \\
\hline $14 \mathrm{~F}$ & 52.15 & 26.72 & 1.07 & $<0.03$ & \\
\hline $15 \mathrm{~A}$ & 54.5 & 14.24 & 2.57 & 0.267 & 0.674 \\
\hline $16 \mathrm{~A}$ & 57.45 & 14.91 & 4.48 & $<0.1$ & \\
\hline \multicolumn{6}{|c|}{ Hanford H1a Sand Unit } \\
\hline $17 \mathrm{~J}$ & 61.75 & 11.63 & 2.01 & $<0.1$ & \\
\hline $17 \mathrm{G}$ & 63.25 & 16.07 & 0.33 & 0.083 & 0.004 \\
\hline $17 \mathrm{G}$ & 63.25 & 14.44 & 2.04 & $<0.1$ & \\
\hline $17 \mathrm{~F}$ & 63.75 & 15.04 & 2.32 & 0.169 & 0.103 \\
\hline 17F-dup & 63.75 & 13.4 & 11.05 & $<0.1$ & \\
\hline $17 \mathrm{DE}$ & 64.75 & 14.99 & 2.35 & 0.131 & 0.126 \\
\hline $17 \mathrm{C}$ & 65.25 & 14.29 & 2.24 & $<0.1$ & \\
\hline $18 \mathrm{I}$ & 67.45 & 15.84 & 0.55 & 0.209 & 0.04 \\
\hline $18 \mathrm{I}$ & 67.45 & 16.44 & 2.37 & 0.22 & 0.126 \\
\hline $18 \mathrm{E}$ & 69.45 & 16.27 & 0.33 & 0.186 & 0.053 \\
\hline $18 \mathrm{E}$ & 69.45 & 16.21 & 5.37 & 0.603 & 0.154 \\
\hline $18 \mathrm{D}$ & 69.95 & 13.58 & 0.56 & $<0.03$ & \\
\hline 18D-dup & 69.95 & 13.07 & 0.55 & $<0.03$ & \\
\hline $18 \mathrm{CB}$ & 70.7 & 12.91 & 0.22 & 0.085 & 0.036 \\
\hline $18 \mathrm{CB}$ & 70.7 & 16.57 & 2.4 & 0.329 & 0.095 \\
\hline 18CB-dup & 70.7 & 15.81 & 2.13 & $<0.1$ & \\
\hline $19 \mathrm{G}$ & 73.75 & 19.66 & 0.95 & $<0.03$ & \\
\hline $19 \mathrm{~F}$ & 74.25 & 16.4 & 0.26 & 0.093 & 0.006 \\
\hline $19 \mathrm{~F}$ & 74.25 & 16.6 & 1.66 & 0.32 & 0.1 \\
\hline \multicolumn{6}{|c|}{ Hanford H1 Coarse Sand/Gravel Unit } \\
\hline $20 \mathrm{E}$ & 79.75 & 35.69 & 1.38 & $<0.03$ & \\
\hline $20 \mathrm{D}$ & 80.25 & 19.86 & 2.42 & $<0.1$ & \\
\hline $22 \mathrm{D}$ & 88.1 & 17.53 & 2.56 & 0.119 & 0.033 \\
\hline $22 \mathrm{~A}$ & 91.1 & 18.64 & 0.56 & $<0.03$ & \\
\hline \multicolumn{6}{|c|}{ Hanford H2 Fine Laminated Sand Unit } \\
\hline $24 \mathrm{~A}$ & 95 & 17.1 & 0.69 & 0.06 & 0.006 \\
\hline
\end{tabular}




\begin{tabular}{|c|c|c|c|c|c|}
\hline \multirow[b]{2}{*}{ Sample ID } & \multirow{2}{*}{$\begin{array}{c}\text { Depth } \\
(\mathbf{f t} \text { bgs) } \\
\end{array}$} & ${ }^{40} \mathbf{K}$ & \pm uncertainty & ${ }^{137} \mathrm{Cs}$ & \pm uncertainty \\
\hline & & \multicolumn{2}{|c|}{$(\mathrm{pCi} / \mathrm{g})$} & \multicolumn{2}{|c|}{$(\mathrm{pCi} / \mathrm{g})$} \\
\hline 24A-dup & 95 & 19.57 & 0.39 & 0.13 & 0.102 \\
\hline $24 \mathrm{~A}$ & 95 & 17.03 & 2.02 & $<0.1$ & \\
\hline $25 \mathrm{~A}$ & 100.06 & 22.34 & 0.96 & $<0.03$ & \\
\hline 27I & 106.05 & 37.41 & 1.25 & $<0.03$ & \\
\hline $27 \mathrm{G}$ & 107.05 & 21.77 & 2.04 & 0.12 & 0.04 \\
\hline 29DC & 116.35 & & & $<0.1$ & \\
\hline \multicolumn{6}{|c|}{ Plio-Pleistocene Fine-Grained Mud Unit } \\
\hline 30GF & 123.85 & 19.41 & 2.25 & 0.111 & 0.042 \\
\hline $30 \mathrm{~B}$ & 126.35 & 20.15 & 0.9 & 0.093 & 0.036 \\
\hline $31 \mathrm{G}$ & 129.15 & 20.11 & 2.56 & $<0.1$ & \\
\hline $31 \mathrm{~A}$ & 132.15 & 18.03 & 0.62 & $<0.03$ & \\
\hline $32 \mathrm{~A}$ & 135.8 & 21.43 & 2.76 & $<0.1$ & \\
\hline $33 \mathrm{G}$ & 137.65 & & & 0.185 & 0.009 \\
\hline $33 \mathrm{G}$ & 137.65 & 18.4 & 1.61 & 0.208 & 0.18 \\
\hline $33 \mathrm{~B}$ & 140.15 & 16.92 & 2.07 & $<0.1$ & \\
\hline $35 \mathrm{~K}$ & 141.05 & 18.5 & 2.19 & $<0.1$ & \\
\hline $35 \mathrm{IH}$ & 141.85 & 24.25 & 2.27 & $<0.1$ & \\
\hline $35 \mathrm{~F}$ & 143.35 & 21.38 & 2.39 & 0.34 & 0.141 \\
\hline $35 \mathrm{C}$ & 144.85 & 21.51 & 2.27 & $<0.1$ & \\
\hline $35 \mathrm{~A}$ & 145.85 & 23.68 & 3 & $<0.1$ & \\
\hline $36 \mathrm{~B}$ & 148.45 & 24.48 & 1.84 & 0.239 & 0.116 \\
\hline 37D & 149.65 & 18.26 & 0.34 & 0.068 & 0.01 \\
\hline 37D & 149.65 & 16.24 & 2.35 & 0.322 & 0.093 \\
\hline $38 \mathrm{E}$ & 153.1 & 17.68 & 2.22 & 0.186 & 0.135 \\
\hline 38E-dup & 153.1 & 16.46 & 3.08 & 0.172 & 0.088 \\
\hline $38 \mathrm{~A}$ & 155.1 & 15.08 & 1.95 & 0.402 & 0.092 \\
\hline $39 \mathrm{C}$ & 155.85 & 10.28 & 14.95 & $<0.1$ & \\
\hline 39C-dup & 155.85 & 9.28 & 1.49 & 0.069 & 0.048 \\
\hline \multicolumn{6}{|c|}{ Plio-Pleistocene Carbonate Unit } \\
\hline $40 \mathrm{~B}$ & 157.75 & 11.41 & 1.98 & 0.202 & 0.073 \\
\hline \multicolumn{6}{|c|}{ Ringlod Formation Unit E } \\
\hline $41 \mathrm{~A}$ & 184.45 & 17 & 1.77 & $<0.1$ & \\
\hline 42 & 194.55 & 19.65 & 0.4 & 0.09 & 0.01 \\
\hline 42 & 194.55 & 16.22 & 3.6 & $<0.1$ & \\
\hline 43B & 204.5 & 18.94 & 1.87 & $<0.1$ & \\
\hline
\end{tabular}




\begin{tabular}{|c|c|c|c|c|c|}
\hline \multirow[b]{2}{*}{ Sample ID } & \multirow{2}{*}{$\begin{array}{c}\text { Depth } \\
\text { (ft bgs) }^{\mathrm{a}}\end{array}$} & ${ }^{40} \mathrm{~K}$ & \pm uncertainty & ${ }^{137} \mathrm{Cs}$ & \pm uncertainty \\
\hline & & \multicolumn{2}{|c|}{$(\mathrm{pCi} / \mathrm{g})$} & \multicolumn{2}{|c|}{$(\mathrm{pCi} / \mathrm{g})$} \\
\hline
\end{tabular}

(a) multiply by 0.3048 to convert to meters

Dup $=$ duplicate sample.

Yellow shading represents small samples counted for short time periods during scouting measurements. The detection limits and counting uncertainties are higher for these samples than for large and longer counted samples. Values in red are those values of cesium-137 that exceed background.

Table 4.12 Strontium-90 and Tritium Contents of Vadose Zone Sediment ${ }^{\mathrm{a}}$

\begin{tabular}{|c|c|c|c|c|c|c|}
\hline $\begin{array}{c}\text { Sample } \\
\text { ID }\end{array}$ & $\begin{array}{c}\text { Depth } \\
\text { (ft bgs) }^{\mathrm{a}}\end{array}$ & Strata & $\begin{array}{c}{ }^{90} \mathrm{Sr} \\
(\mathrm{pCi} / \mathrm{g})\end{array}$ & $\begin{array}{c}\text { Uncertainty } \\
\text { "+/-" }\end{array}$ & $\begin{array}{c}{ }^{3} \mathbf{H} \\
(\mathrm{pCi} / \mathrm{g})\end{array}$ & $\begin{array}{c}\text { Uncertainty } \\
\text { "+/-" }\end{array}$ \\
\hline $13 \mathrm{~A}$ & 46.85 & H1a & & & 1.9 & 1.6 \\
\hline 13A-dup & 46.85 & H1a & & & 1.2 & 0.0 \\
\hline $17 \mathrm{DE}$ & 64.75 & H1a & -2.43 & 2.77 & & \\
\hline 17DE-dup & 64.75 & H1a & 0.21 & 2.74 & & \\
\hline $18 \mathrm{E}$ & 69.45 & $\mathrm{H} 1 \mathrm{a}$ & 1.07 & 2.76 & & \\
\hline $18 \mathrm{D}$ & 69.95 & H1a & & & 8.2 & 1.8 \\
\hline $18 \mathrm{BC}$ & 70.70 & H1a & 0.09 & 2.79 & & \\
\hline 18BC-dup & 70.70 & H1a & 1.46 & 2.77 & & \\
\hline $19 \mathrm{H}$ & 73.25 & $\mathrm{H} 1 \mathrm{a}$ & 1.78 & 3.06 & & \\
\hline $19 \mathrm{G}$ & 73.75 & H1a & 0.00 & 2.75 & 32.0 & 2.5 \\
\hline $30 \mathrm{D}$ & 125.35 & $\mathrm{PPlz}$ & & & 139.8 & 7.0 \\
\hline $31 \mathrm{G}$ & 129.15 & $\mathrm{PPlz}$ & 1.72 & 2.86 & & \\
\hline $32 \mathrm{D}$ & 134.25 & $\mathrm{PPlz}$ & & & 25.6 & 2.3 \\
\hline $36 \mathrm{D}$ & 147.45 & $\mathrm{PPlz}$ & & & 2.4 & 1.1 \\
\hline 36D-dup & 147.45 & $\mathrm{PPlz}$ & & & 3.6 & 1.4 \\
\hline $38 \mathrm{G}$ & 152.1 & $\mathrm{PPlz}$ & -0.93 & 2.71 & 4.3 & 1.6 \\
\hline $38 \mathrm{~A}$ & 155.10 & $\mathrm{PPlz}$ & 1.52 & 2.86 & & \\
\hline $39 \mathrm{D}$ & 155.4 & $\mathrm{PPlz}$ & 0.00 & 2.75 & 2.6 & 1.0 \\
\hline \multicolumn{7}{|c|}{$\begin{array}{l}\text { (a) multiply by } 0.3048 \text { to convert to meters } \\
\text { The strontium- } 90 \text { data are all below detection limits and suggest no measureable strontium-90 in the } \\
\text { borehole sediment. }\end{array}$} \\
\hline
\end{tabular}

Table 4.13 Technetium-99 and Uranium Content in Sediment (based on 8 M Nitric Acid extracts)

\begin{tabular}{|c|c|c|c|c||}
\hline \hline Sample ID & $\begin{array}{c}\text { Depth } \\
\text { (ft bgs) }^{\mathbf{a}}\end{array}$ & $\begin{array}{c}\text { Tc 99 } \\
\text { (pCi/g soil) }^{\text {p }}\end{array}$ & $\begin{array}{c}\text { U 238 } \\
\text { (ug/g soil) }\end{array}$ & $\begin{array}{c}\text { U 238 } \\
\text { (pCi/g soil) }^{\text {(u) }}\end{array}$ \\
\hline \hline 17DE & 64.75 & $<5.43$ & 0.853 & 0.287 \\
\hline 17DE-dup & 64.75 & $<2.51$ & 0.830 & 0.279 \\
\hline
\end{tabular}




\begin{tabular}{|c|c|c|c|c||}
\hline $18 \mathrm{E}$ & 69.45 & $<5.04$ & 0.532 & 0.179 \\
\hline $18 \mathrm{CB}$ & 70.70 & $<8.80$ & 0.505 & 0.170 \\
\hline $18 \mathrm{CB}-$ dup & 70.70 & $<10.67$ & 0.553 & 0.186 \\
\hline $19 \mathrm{H}$ & 73.25 & 17.17 & 0.420 & 0.141 \\
\hline $19 \mathrm{G}$ & 73.75 & 46.02 & 0.703 & 0.237 \\
\hline $20 \mathrm{E}$ & 79.75 & 31.22 & 0.824 & 0.277 \\
\hline $26 \mathrm{~A}$ & 104.95 & $<11.52$ & 0.569 & 0.191 \\
\hline $31 \mathrm{G}$ & 129.15 & 547.56 & 1.139 & 0.383 \\
\hline $31 \mathrm{~A}$ & 132.15 & 341.82 & 0.912 & 0.307 \\
\hline $36 \mathrm{D}$ & 147.45 & 114.50 & 0.866 & 0.291 \\
\hline $38 \mathrm{G}$ & 152.10 & 61.67 & 1.176 & 0.396 \\
\hline $38 \mathrm{~A}$ & 155.10 & $<9.64$ & 1.350 & 0.454 \\
\hline $39 \mathrm{D}$ & 155.35 & 46.45 & 0.830 & 0.279 \\
\hline
\end{tabular}

(a) multiply by 0.3048 to convert to meters 


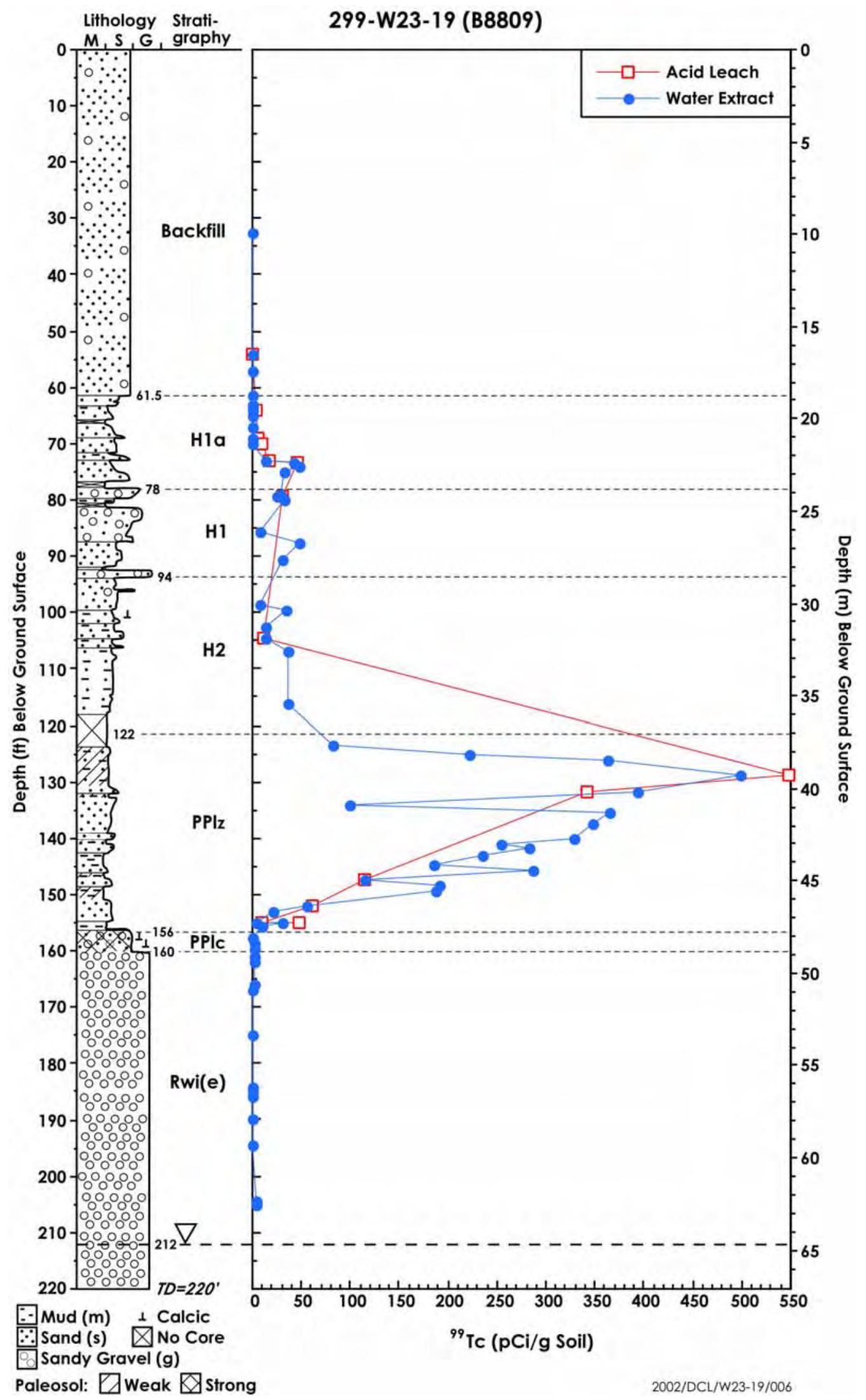

Figure 4.6 Water- and Acid-Extractable Technetium-99 in Sediment from Borehole 299-W23-19. 


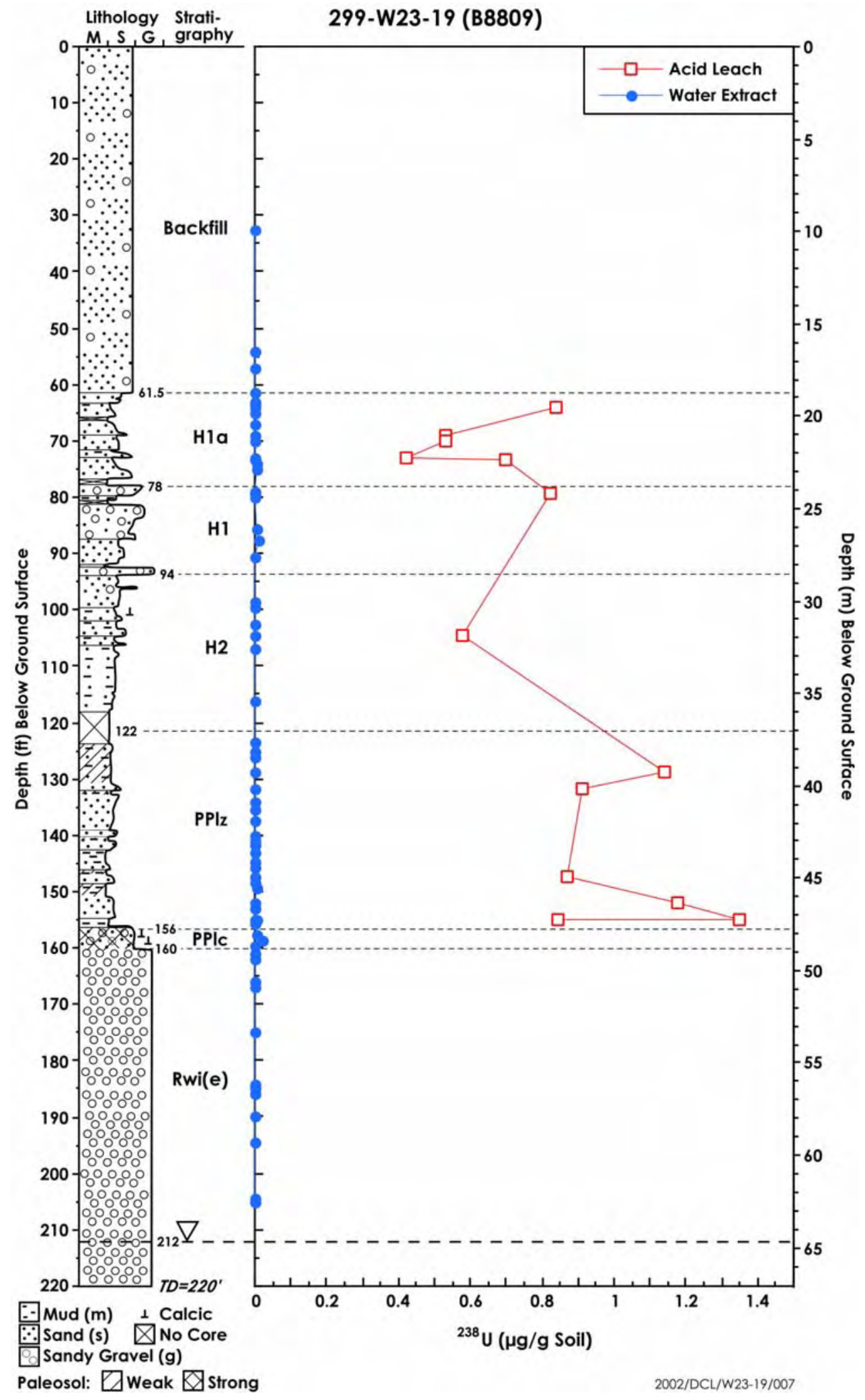

Figure 4.7 Uranium Extracted from Sediment (water versus acid). 


\subsubsection{M Nitric Acid-Extractable Amounts of Selected Elements}

Tables 4.15 and 4.16 show the concentrations of various elements extracted from the vadose zone sediment by $8 \mathrm{M}$ nitric acid. The data show that the acid-extractable concentrations of calcium, magnesium, sodium, strontium, and perhaps chromium and manganese are greater than those from sediment in an uncontaminated borehole (Serne et al. 2002a). The quantities of acid-extractable sodium and chromium reflect the presence of dilute REDOX waste but the acid-extractable concentrations for the other constituents are not indicative of leaked tank liquor. For most elements (aluminum, arsenic, cadmium, calcium, iron, lead, magnesium, manganese, potassium, silver, strontium, and zinc), the amount that is water-extractable is a very small fraction of that which is acid-extractable. The comparison of water leachable versus acid leachable concentrations is a qualitative estimate of species mobility. More discussion of this is found in Section 5. 
Table 4.14 Carbon Content in Vadose Sediment from 299-W23-19 Borehole

\begin{tabular}{|c|c|c|c|c|c|c|c|c|c|c|c|}
\hline \multirow[b]{2}{*}{ ID } & \multirow[b]{2}{*}{$\begin{array}{c}\text { Depth } \\
(\text { ft } \text { bgs })^{\mathrm{a}}\end{array}$} & \multirow{2}{*}{$\begin{array}{c}\text { Total } \\
\text { Carbon } \\
(w t \%)\end{array}$} & \multicolumn{2}{|c|}{\begin{tabular}{|l|l|} 
Inorganic & Carbonic as \\
Inorbon
\end{tabular}} & Organic & \multirow[b]{2}{*}{ ID } & \multirow[b]{2}{*}{$\begin{array}{c}\text { Depth } \\
(\text { ft bgs) }\end{array}$} & \multirow{2}{*}{$\begin{array}{c}\text { Total } \\
\text { Carbon } \\
(w \mathrm{t} \%)\end{array}$} & \multirow{2}{*}{$\begin{array}{c}\text { Inorgani } \\
\text { Carbon } \\
(w t \%)\end{array}$} & $\begin{array}{l}\text { Inorganic } \\
\text { Carbon as }\end{array}$ & \multirow{2}{*}{$\begin{array}{l}\text { Organi } \\
\text { Carbon } \\
(\mathrm{wt} \%)\end{array}$} \\
\hline & & & $\begin{array}{c}\text { Carbon } \\
(w t \%)\end{array}$ & $\begin{array}{c}\mathrm{CaCO}_{3} \\
(\mathrm{wt} \%)\end{array}$ & $\begin{array}{c}\text { Carbon } \\
(\mathrm{wt} \%)\end{array}$ & & & & & $\begin{array}{c}\mathrm{CaCO}_{3} \\
(\mathrm{wt} \%)\end{array}$ & \\
\hline & & & & & & \multicolumn{6}{|c|}{ PPlz } \\
\hline \multicolumn{6}{|c|}{ Backfill } & $30 \mathrm{GF}$ & 124.1 & 0.38 & 0.33 & 2.73 & 0.05 \\
\hline $1 \mathrm{D}$ & 7.55 & 0.22 & 0.18 & 1.49 & 0.05 & $30 \mathrm{D}$ & 125.35 & 0.42 & 0.37 & 3.05 & 0.05 \\
\hline $3 \mathrm{I}$ & 12.35 & 0.21 & 0.15 & 1.24 & 0.06 & 30B & 126.35 & 0.48 & 0.38 & 3.21 & 0.09 \\
\hline $9 \mathrm{E}$ & 32.95 & 0.22 & 0.18 & 1.49 & 0.04 & $31 \mathrm{G}$ & 129.15 & 0.51 & 0.46 & 3.86 & 0.04 \\
\hline $11 \mathrm{C}$ & 42.35 & 0.2 & 0.17 & 1.42 & 0.03 & $32 \mathrm{D}$ & 134.25 & 0.43 & 0.36 & 2.99 & 0.07 \\
\hline $13 \mathrm{~A}$ & 46.85 & 0.22 & 0.24 & 1.96 & -0.01 & $32 \mathrm{~A}$ & 135.8 & 0.56 & 0.5 & 4.13 & 0.06 \\
\hline $15 \mathrm{~A}$ & 54.5 & 0.31 & 0.15 & 1.27 & 0.16 & $33 \mathrm{G}$ & 137.65 & 0.41 & 0.35 & 2.88 & 0.06 \\
\hline $16 \mathrm{~A}$ & 57.45 & 0.2 & 0.16 & 1.3 & 0.04 & $33 \mathrm{~B}$ & 140.15 & 0.47 & 0.44 & 3.64 & 0.03 \\
\hline \multicolumn{6}{|c|}{ H1a } & $35 \mathrm{~K}$ & 141 & 0.46 & 0.42 & 3.5 & 0.04 \\
\hline $17 \mathrm{~J}$ & 61.75 & 0.17 & 0.12 & 1.01 & 0.05 & $35 \mathrm{IH}$ & 142.1 & 0.57 & 0.52 & 4.32 & 0.05 \\
\hline $17 \mathrm{G}$ & 63.25 & 0.23 & 0.18 & 1.47 & 0.05 & $35 \mathrm{~F}$ & 143.35 & 0.61 & 0.54 & 4.51 & 0.07 \\
\hline $17 \mathrm{~F}$ & 63.75 & 0.38 & 0.32 & 2.64 & 0.06 & $35 \mathrm{~A}$ & 145.85 & 0.45 & 0.42 & 3.49 & 0.03 \\
\hline 17DE & 64.5 & 0.38 & 0.3 & 2.53 & 0.08 & $36 \mathrm{D}$ & 147.45 & 0.34 & 0.27 & 2.28 & 0.07 \\
\hline $17 \mathrm{C}$ & 65.25 & 0.32 & 0.25 & 2.12 & 0.07 & $36 \mathrm{~B}$ & 148.45 & 0.56 & 0.46 & 3.87 & 0.09 \\
\hline $18 \mathrm{I}$ & 67.45 & 0.31 & 0.25 & 2.06 & 0.06 & $37 \mathrm{D}$ & 149.65 & 0.44 & 0.34 & 2.86 & 0.09 \\
\hline $18 \mathrm{E}$ & 69.45 & 0.28 & 0.24 & 1.97 & 0.05 & $38 \mathrm{G}$ & 152.1 & 0.49 & 0.44 & 3.63 & 0.06 \\
\hline $18 \mathrm{D}$ & 69.95 & 0.25 & 0.22 & 1.8 & 0.03 & $38 \mathrm{E}$ & 153.1 & 0.42 & 0.36 & 3 & 0.06 \\
\hline $18 \mathrm{CB}$ & 70.7 & 0.24 & 0.18 & 1.48 & 0.06 & $38 \mathrm{~A}$ & 155.1 & 0.31 & 0.24 & 1.97 & 0.07 \\
\hline $19 \mathrm{G}$ & 73.75 & 0.27 & 0.23 & 1.9 & 0.04 & 39D & 155.35 & 0.39 & 0.35 & 2.89 & 0.04 \\
\hline $19 \mathrm{~F}$ & 74.25 & 0.3 & 0.26 & 2.17 & 0.04 & $39 \mathrm{C}$ & 155.85 & 0.26 & 0.22 & 1.87 & 0.04 \\
\hline \multicolumn{6}{|c|}{ H1 } & \multicolumn{6}{|c|}{ PPlc } \\
\hline $20 \mathrm{D}$ & 80.25 & 0.27 & 0.21 & 1.75 & 0.06 & $40 \mathrm{~B}$ & 157.75 & 0.14 & 0.09 & 0.75 & 0.05 \\
\hline $22 \mathrm{D}$ & 88.1 & 0.24 & 0.2 & 1.7 & 0.04 & 40 & 158.65 & 3.16 & 3 & 24.97 & 0.17 \\
\hline \multicolumn{6}{|c|}{ H2 } & \multicolumn{6}{|c|}{ Rwi(e) } \\
\hline $24 \mathrm{~A}$ & 95 & 0.25 & 0.22 & 1.85 & 0.03 & $41 \mathrm{~A}$ & 184.45 & 0.04 & 0.01 & 0.08 & 0.03 \\
\hline $27 \mathrm{G}$ & 107.05 & 0.26 & 0.23 & 1.92 & 0.03 & 42 & 194.55 & 0.07 & 0.01 & 0.08 & 0.06 \\
\hline $29 \mathrm{DC}$ & 108.77 & 0.29 & 0.25 & 2.1 & 0.04 & $43 B$ & 204.5 & 0.05 & 0.01 & 0.08 & 0.04 \\
\hline & & & & & & $43 \mathrm{~A}$ & 205.15 & 0.07 & 0.02 & 0.16 & 0.05 \\
\hline
\end{tabular}

(a) multiply by 0.3048 to convert to meters 
Table 4.15 Acid-Extractable Major Element Concentrations of the Vadose Sediment from Borehole 299-W23-19

\begin{tabular}{|c|c|c|c|c|c|c|c|c|c|c|c|c|}
\hline $\begin{array}{c}\text { Sample } \\
\text { ID }\end{array}$ & $\begin{array}{c}\text { Depth } \\
(\text { ft bgs })^{a}\end{array}$ & $\begin{array}{c}\text { Al } \\
\text { (ug/g soil) }\end{array}$ & $\begin{array}{c}\text { Na } \\
\text { (ug/g soil) }\end{array}$ & $\begin{array}{c}\text { K } \\
\text { (ug/g soil) }\end{array}$ & $\begin{array}{c}\text { Ca } \\
\text { (ug/g soil) }\end{array}$ & $\begin{array}{c}\text { Mg } \\
\text { (ug/g soil) }\end{array}$ & $\begin{array}{c}\mathrm{Sr} \\
\text { (ug/g soil) }\end{array}$ & $\begin{array}{c}\text { Ba } \\
\text { (ug/g soil) }\end{array}$ & $\begin{array}{c}\text { Fe } \\
\text { (ug/g soil) }\end{array}$ & $\begin{array}{c}\text { Mn } \\
\text { (ug/g soil) } \\
\end{array}$ & $\begin{array}{c}\text { Zn } \\
\text { (ug/g soil) }\end{array}$ & $\begin{array}{c}\text { Ni } \\
\text { (ug/g soil) }\end{array}$ \\
\hline $17 \mathrm{DE}$ & 64.75 & 15,712 & -- & 2197 & 19,176 & 8395 & 83.8 & 168.0 & 29,449 & 608.4 & 60.3 & 17.9 \\
\hline 17DE-dup & 64.75 & 15,630 & --- & 2312 & 19,736 & 8782 & 89.6 & 177.9 & 31,406 & 687.6 & 64.9 & 19.3 \\
\hline $18 \mathrm{E}$ & 69.45 & 11,180 & --- & 1766 & 11,973 & 6078 & 60.6 & 102.3 & 19,689 & 406.6 & 48.8 & 14.1 \\
\hline $18 \mathrm{CB}$ & 70.70 & 10,793 & --- & 1533 & 11,175 & 5943 & 59.4 & 101.3 & 18,956 & 391.8 & 46.8 & 14.8 \\
\hline 18CB-dup & 70.70 & 12,078 & --- & 1801 & 11,989 & 6429 & 65.1 & 117.7 & 20,843 & 430.8 & 51.1 & 15.6 \\
\hline $19 \mathrm{H}$ & 73.25 & 6,315 & --- & 851 & 7,944 & 4721 & 37.7 & 66.6 & 14,718 & 280.8 & 34.9 & 11.5 \\
\hline $19 \mathrm{G}$ & 73.75 & 10,185 & $\ldots$ & 1875 & 10,943 & 6223 & 50.5 & 109.2 & 19,035 & 393.1 & 53.1 & 13.5 \\
\hline $20 \mathrm{E}$ & 79.75 & 13,046 & $\ldots$ & 2600 & 10,730 & 7201 & 59.3 & 140.1 & 23,237 & 453.7 & 61.3 & 13.7 \\
\hline $26 \mathrm{~A}$ & 104.95 & 8,243 & --- & 1454 & 9,725 & 5686 & 46.2 & 88.7 & 15,683 & 353.8 & 42.9 & 14.6 \\
\hline $31 \mathrm{G}$ & 129.15 & 12,117 & --- & 1962 & 14,227 & 7623 & 56.9 & 111.0 & 19,305 & 288.7 & 58.3 & 17.6 \\
\hline $31 \mathrm{~A}$ & 132.15 & 15,373 & $--\cdot$ & 2510 & 19,017 & 8931 & 77.0 & 133.7 & 22,054 & 382.6 & 65.2 & 19.0 \\
\hline $36 \mathrm{D}$ & 147.45 & 12,309 & -- & 2128 & 12,732 & 7871 & 56.9 & 135.2 & 20,908 & 372.0 & 60.2 & 17.1 \\
\hline $38 \mathrm{G}$ & 152.10 & 15,427 & -- & 2381 & 16,550 & 9373 & 53.0 & 142.7 & 22,500 & 430.5 & 66.6 & 17.9 \\
\hline $38 \mathrm{~A}$ & 155.10 & 18,509 & -- & 2459 & 14,207 & 8927 & 61.8 & 162.6 & 31,126 & 405.3 & 71.8 & 20.8 \\
\hline $39 \mathrm{D}$ & 155.35 & 9,437 & --- & 1385 & 16,218 & 5619 & 53.8 & 117.0 & 25,843 & 384.3 & 54.7 & 11.2 \\
\hline
\end{tabular}

(a) multiply by 0.3048 to convert to meters 
Table 4.16 Acid-Extractable Trace Element Concentrations of the Vadose Sediment from Borehole 299-W23-19.

\begin{tabular}{|c|c|c|c|c|c|c|c|c|c|c|c|c|}
\hline \multirow[b]{2}{*}{$\begin{array}{c}\text { Sample } \\
\text { ID }\end{array}$} & \multirow{2}{*}{$\begin{array}{c}\text { Depth } \\
\text { (ft bgs) }^{\text {a }}\end{array}$} & $\mathrm{Cr}^{*}$ & $\mathrm{Cr}$ & Co* & $\mathbf{C u}^{*}$ & As & Se & Mo & Ag & Cd & $\mathbf{P b}^{*}$ & $\mathbf{P b}$ \\
\hline & & \multicolumn{11}{|c|}{ (ug/g soil) } \\
\hline $17 \mathrm{DE}$ & 64.75 & 16.7 & 17.2 & 14.9 & 14.3 & 3.2 & $<0.04$ & 0.08 & 0.09 & 0.11 & 5.4 & 7.7 \\
\hline 17DE-dup & 64.75 & 17.7 & 18.7 & 16.5 & 10.6 & 3.5 & $<0.06$ & $<0.10$ & 0.10 & 0.12 & 5.6 & 8.9 \\
\hline $18 \mathrm{E}$ & 69.45 & 22.0 & 25.9 & 10.0 & 10.7 & 3.0 & $<0.04$ & 0.09 & 0.06 & 0.08 & 3.2 & 5.4 \\
\hline $18 \mathrm{CB}$ & 70.70 & 25.7 & 18.0 & 9.5 & 10.9 & 3.1 & $<0.05$ & 0.11 & 0.05 & 0.06 & $(2.7)$ & 4.2 \\
\hline 18CB-dup & 70.70 & 27.0 & 28.7 & 10.2 & 16.1 & 3.4 & $<0.06$ & $<0.12$ & 0.06 & 0.08 & $(2.9)$ & 5.1 \\
\hline $19 \mathrm{H}$ & 73.25 & 17.7 & 19.1 & 6.5 & 8.3 & 2.5 & 0.07 & 0.17 & 0.05 & 0.06 & $(2.3)$ & 3.3 \\
\hline $19 \mathrm{G}$ & 73.75 & 33.4 & 38.8 & 9.3 & 9.4 & 4.0 & $<0.05$ & 0.21 & 0.06 & 0.07 & 4.9 & 6.8 \\
\hline $20 \mathrm{E}$ & 79.75 & 30.6 & 33.9 & 11.6 & 10.8 & 6.1 & $<0.02$ & 0.14 & 0.07 & 0.09 & 6.3 & 8.6 \\
\hline $26 \mathrm{~A}$ & 104.95 & 24.0 & 26.6 & 7.8 & 9.6 & 4.3 & $<0.06$ & 0.39 & 0.05 & 0.09 & 4.1 & 5.8 \\
\hline $31 \mathrm{G}$ & 129.15 & 18.2 & 19.6 & 10.2 & 16.5 & 3.3 & $<0.04$ & 0.08 & 0.08 & 0.14 & 7.4 & 10.0 \\
\hline $31 \mathrm{~A}$ & 132.15 & 21.0 & 22.1 & 11.4 & 18.3 & 3.5 & $<0.05$ & 0.09 & 0.09 & 0.16 & 8.6 & 12.2 \\
\hline $36 \mathrm{D}$ & 147.45 & 19.5 & 20.5 & 11.0 & 13.5 & 4.9 & $<0.05$ & 0.11 & 0.07 & 0.12 & 7.0 & 9.4 \\
\hline $38 \mathrm{G}$ & 152.10 & 18.6 & 20.3 & 11.7 & 19.2 & 6.9 & $<0.05$ & 0.10 & 0.09 & 0.15 & 10.1 & 14.4 \\
\hline $38 \mathrm{~A}$ & 155.10 & 22.6 & 24.6 & 16.2 & 19.1 & 7.2 & $<0.07$ & 0.11 & 0.12 & 0.15 & 6.4 & 10.7 \\
\hline $39 \mathrm{D}$ & 155.35 & 14.9 & 16.4 & 12.2 & 13.6 & 6.9 & 0.13 & 0.98 & 0.10 & 0.13 & 4.9 & 6.9 \\
\hline \multicolumn{13}{|c|}{$\begin{array}{l}\text { (a) multiply by } 0.3048 \text { to convert to meters } \\
\text { * Measurement performed by ICP; all others (unmarked) performed by ICP-MS. Agreement for the two analytical methods for chromium and lead is } \\
\text { excellent }\end{array}$} \\
\hline
\end{tabular}




\subsubsection{Sediment Total Oxide Composition}

Several samples of the bulk vadose zone sediment were crushed and analyzed by x-ray fluorescence to obtain oxide concentration in the sediment. Additional aliquots of these same sediments were subjected to particle-size analysis from which the sand, silt, and clay separates were retained. The claysize fraction $(<2 \mu \mathrm{m})$ was also analyzed by $\mathrm{x}$-ray fluorescence to measure oxide concentrations. The total oxide composition of both the bulk and clay-size separates was used to aid in the quantification of mineralogy that will be discussed later.

By using the $x$-ray fluorescence most natural element concentrations were obtained. The capability for measuring the concentrations of only beryllium, boron, fluorine, hydrogen, lithium, and nitrogen was not available for this study. Carbon content was obtained from samples of bulk sediment as discussed in Section 4.2.4. The concentrations of beryllium, boron, fluorine, hydrogen, lithium, and nitrogen, in the sediment is likely small so that a calculated oxide mass for the sediment should come close to $100 \mathrm{wt} \%$ without these elements. All iron in the sediment is assumed to be ferric oxide, although some ferrous iron is expected in the mafic components.

The results of the x-ray fluorescence analyses of the bulk sediment are shown in Table 4.17 and, for the clay separates, in Table 4.18. The oxide totals for the bulk sediment vary from 93.4 to $111.8 \mathrm{wt} \%$. The sample totaling $112 \mathrm{wt} \%$ is from a sample of the Ringold Formation unit E, which shows excessive silica resulting in the high total. In general, the Hanford formation sediment from borehole 299-W23-19 is dominated by silica and alumina, as expected. Calcium, carbonate, iron, magnesium, potassium, sodium, and titanium make up most of the remainder of the sediment. There is no large database of Hanford formation oxide compositions against which to compare the results from borehole 299-W23-19. However, a few results are available from nearby, uncontaminated boreholes (Serne et al. 2002). The contaminated sediment in the Hanford formation H1a unit in borehole 299-W23-19 is slightly depleted in aluminum oxide, sodium oxide, and magnesium oxide as compared to sediment in the uncontaminated boreholes. For the coarser-grained Hanford formation H1 unit, the contaminated sediments are slightly lower in aluminum oxide, magnesium oxide, calcium oxide, titanium oxide, and ferric oxide and higher in potassium oxide and silicon oxide than are the uncontaminated sediments. These differences may be due to differences in the amounts of basalt and felsic components in the sediment and not to contamination. The Hanford formation H2 unit in borehole 299-W23-19 is slightly lower in aluminum oxide and magnesium oxide than is the Hanford formation $\mathrm{H} 2$ unit in the uncontaminated borehole.

The upper Plio-Pleistocene unit from borehole 299-W23-19 does not differ in oxide composition from that in the nearby uncontaminated borehole. However, the data in Table 4.17 show that the upper PlioPleistocene unit is depleted in sodium oxide, titanium oxide, and ferric oxide and enriched in potassium oxide and magnesium oxide relative to the deeper samples from the lower Plio-Pleistocene unit just above the Ringold Formation (samples $31 \mathrm{G}$ versus 38A and 39D).

The only oxide that might show elevated mass due to contamination in the bulk sediment is sodium

oxide. However, the sodium oxide content of each lithology in borehole 299-W23-19 is not significantly greater than sodium oxide contents for the same strata in the two uncontaminated boreholes (Serne et al. 2002a). As will be discussed in Section 5, the acid extracts do show differences between the sediment in the contaminated and uncontaminated boreholes. 
The oxide compositions for the clay-size separates are shown in Table 4.18. The carbon value in that table is from the bulk sediment analysis of total carbon. The clay fraction may contain more carbon than the bulk sediment because of the fine-grained habit of the calcite in the samples. Thus, the total mass is probably greater than what is shown in the table. The clay-sized material consistently has higher concentrations of ferric oxide, magnesium oxide, and aluminum oxide and lower concentrations of calcium oxide, sodium oxide, and silicon oxide than the bulk sediment. This undoubtedly reflects the higher quartz and feldspar content of the bulk sediment and the probable higher concentrations of iron and aluminum oxyhydroxides.

Because the x-ray fluorescence analysis gives totals near $100 \mathrm{wt} \%$, they probably give an accurate measurement of the total sediment oxide composition. The acid extraction dissolves a portion of that total oxide content. Using both of these data sets, the fraction of material dissolved by the acid extraction can be estimated. Table 4.19 lists the percentages of the total sample that was dissolved during acid extraction.

Table 4.17 Oxide Concentrations of Bulk Vadose Zone Sediment from Borehole 299-W23-19 (wt\%)

\begin{tabular}{|c|c|c|c|c|c|c|c|c|}
\hline Sample ID & $17 \mathrm{DE}$ & $19 \mathrm{G}$ & $22 \mathrm{D}$ & $26 \mathrm{~A}$ & $31 \mathrm{G}$ & $38 \mathrm{~A}$ & 39D & $43 \mathrm{~B}$ \\
\hline Depth (ft bgs) ${ }^{a}$ & 64.75 & 73.75 & 88.10 & 104.95 & 129.15 & 155.10 & 155.35 & 204.50 \\
\hline Unit & $\mathrm{H} 1 \mathrm{a}$ & $\mathrm{H} 1 \mathrm{a}$ & $\mathrm{H} 1$ & $\mathrm{H} 2$ & $\mathrm{PPlz}$ & $\mathrm{PPlz}$ & $\mathrm{PPlz}$ & Rwi(e) \\
\hline $\mathrm{CO}_{2}$ & 1.39 & 0.99 & 0.90 & 1.01 & 1.86 & 1.13 & 1.42 & 0.18 \\
\hline $\mathrm{Na}_{2} \mathrm{O}$ & 1.78 & 2.48 & 2.51 & 2.47 & 1.40 & 2.29 & 2.37 & 3.72 \\
\hline $\mathrm{MgO}$ & 2.14 & 2.07 & 2.01 & 1.97 & 2.17 & 2.32 & 2.42 & 1.54 \\
\hline $\mathrm{Al}_{2} \mathrm{O}_{3}$ & 10.45 & 11.71 & 11.13 & 12.15 & 11.66 & 13.81 & 12.55 & 13.38 \\
\hline $\mathrm{SiO}_{2}$ & 62.78 & 72.65 & 71.71 & 71.75 & 67.00 & 70.45 & 61.62 & 83.66 \\
\hline $\mathrm{P}_{2} \mathrm{O}_{5}$ & $<0.30$ & $<0.27$ & $<0.27$ & $<0.27$ & $<0.27$ & 0.41 & 0.61 & $<0.30$ \\
\hline $\mathrm{SO}_{3}$ & 0.12 & $<0.09$ & $<0.08$ & $<0.07$ & $<0.08$ & $<0.09$ & 0.14 & $<0.08$ \\
\hline $\mathrm{Cl}$ & $<0.02$ & 0.05 & 0.06 & 0.04 & 0.05 & 0.03 & $<0.02$ & 0.06 \\
\hline $\mathrm{K}_{2} \mathrm{O}$ & 1.81 & 2.48 & 2.35 & 2.46 & 2.40 & 1.98 & 1.57 & 2.63 \\
\hline $\mathrm{CaO}$ & 5.20 & 3.64 & 3.48 & 3.50 & 3.89 & 4.16 & 6.57 & 2.49 \\
\hline $\mathrm{TiO}_{2}$ & 0.98 & 0.84 & 0.69 & 0.65 & 0.71 & 1.41 & 2.19 & 0.49 \\
\hline $\mathrm{V}_{2} \mathrm{O}_{5}$ & 0.02 & 0.01 & 0.01 & 0.01 & 0.01 & 0.03 & 0.06 & 0.01 \\
\hline $\mathrm{Cr}_{2} \mathrm{O}_{3}$ & 0.01 & 0.01 & 0.01 & 0.01 & 0.01 & 0.01 & 0.01 & 0.01 \\
\hline $\mathrm{MnO}$ & 0.10 & 0.08 & 0.07 & 0.08 & 0.06 & 0.08 & 0.14 & 0.07 \\
\hline $\mathrm{Fe}_{2} \mathrm{O}_{3}$ & 6.10 & 4.87 & 4.22 & 4.11 & 4.29 & 6.20 & 9.27 & 3.41 \\
\hline $\mathrm{SrO}$ & 0.04 & 0.05 & 0.04 & 0.05 & 0.03 & 0.04 & 0.04 & 0.04 \\
\hline $\mathrm{BaO}$ & 0.08 & 0.09 & 0.08 & 0.11 & 0.10 & 0.08 & 0.08 & 0.07 \\
\hline Total & 93.40 & 102.02 & 99.25 & 100.36 & 95.64 & 104.42 & 101.06 & 111.75 \\
\hline
\end{tabular}

(a) multiply by 0.3048 to convert to meters 
Table 4.18 Total Composition of the Clay-Sized Fraction of Sediment from Borehole 299-W23-19 wt\% As Oxides

\begin{tabular}{|c|c|c|c|c|c|c|c|c|}
\hline Sample ID & $17 \mathrm{DE}$ & $19 \mathrm{G}$ & $22 \mathrm{D}$ & $26 \mathrm{~A}$ & $31 \mathrm{G}$ & $38 \mathrm{~A}$ & $39 \mathrm{D}$ & 43B \\
\hline Depth (ft bgs) ${ }^{\mathbf{a}}$ & 64.75 & 73.75 & 88.10 & 104.95 & 129.15 & 155.10 & 155.35 & 204.50 \\
\hline Unit & H1a & $\mathrm{H} 1 \mathrm{a}$ & $\mathrm{H} 1$ & $\mathrm{H} 2$ & $\mathrm{PPlz}$ & $\mathrm{PPlz}$ & $\mathrm{PPlz}$ & Rwi(e) \\
\hline $\mathrm{CO}_{2}$ & 1.11 & 0.84 & 0.75 & 0.88 & 1.70 & 0.87 & 1.27 & 0.04 \\
\hline $\mathrm{Na}_{2} \mathrm{O}$ & 0.70 & 0.81 & 1.17 & 1.25 & 0.85 & 1.01 & 0.84 & 3.11 \\
\hline $\mathrm{MgO}$ & 4.01 & 3.43 & 3.43 & 3.95 & 3.15 & 3.50 & 3.32 & 3.02 \\
\hline $\mathrm{Al}_{2} \mathrm{O}_{3}$ & 10.88 & 16.16 & 16.65 & 18.28 & 16.81 & 17.47 & 16.19 & 14.83 \\
\hline $\mathrm{SiO}_{2}$ & 49.46 & 52.36 & 53.16 & 55.32 & 51.58 & 60.45 & 55.49 & 62.18 \\
\hline $\mathrm{P}_{2} \mathrm{O}_{5}$ & 7.03 & 0.75 & 0.54 & 1.22 & 0.38 & 0.33 & 0.95 & 0.33 \\
\hline $\mathrm{SO}_{3}$ & 0.31 & $<0.06$ & $<0.06$ & 0.08 & $<0.06$ & $<0.06$ & $<0.07$ & 0.09 \\
\hline $\mathrm{Cl}$ & $<0.02$ & 0.04 & 0.05 & 0.05 & 0.04 & 0.03 & 0.04 & 0.03 \\
\hline $\mathrm{K}_{2} \mathrm{O}$ & 1.43 & 2.29 & 2.96 & 3.19 & 2.61 & 2.40 & 2.39 & 2.11 \\
\hline $\mathrm{CaO}$ & 6.26 & 1.31 & 1.10 & 1.48 & 1.78 & 0.97 & 2.02 & 2.16 \\
\hline $\mathrm{TiO}_{2}$ & 0.68 & 0.88 & 0.86 & 1.00 & 0.89 & 1.04 & 0.99 & 0.94 \\
\hline $\mathrm{V}_{2} \mathrm{O}_{5}$ & 0.01 & 0.02 & 0.03 & 0.03 & 0.02 & 0.02 & 0.02 & 0.02 \\
\hline $\mathrm{Cr}_{2} \mathrm{O}_{3}$ & 0.01 & 0.03 & 0.03 & 0.03 & 0.01 & 0.01 & 0.01 & 0.01 \\
\hline $\mathrm{MnO}$ & 0.20 & 0.27 & 0.19 & 0.27 & 0.17 & 0.15 & 0.19 & 0.22 \\
\hline $\mathrm{Fe}_{2} \mathrm{O}_{3}$ & 8.71 & 10.86 & 10.36 & 10.92 & 10.07 & 12.06 & 11.91 & 9.67 \\
\hline $\mathrm{SrO}$ & 0.03 & 0.02 & 0.02 & 0.02 & 0.02 & 0.01 & 0.02 & 0.03 \\
\hline $\mathrm{BaO}$ & 0.08 & 0.10 & 0.14 & 0.14 & 0.12 & 0.10 & 0.16 & 0.09 \\
\hline Total & 91.21 & 90.34 & 91.56 & 98.24 & 90.36 & 100.69 & 95.95 & 99.02 \\
\hline
\end{tabular}

(a) multiply by 0.3048 to convert to meters

Note: The C content of the clay separates was not measured, so the bulk values were used as an estimate.

In general, the acid extract removed 1 to $10 \mathrm{wt} \%$ of the total mass of alkali oxides, about $25 \mathrm{wt} \%$ of the major alkaline earth oxides (magnesium and calcium), and less than 10 to $15 \mathrm{wt} \%$ of the minor alkaline earth oxides (strontium and barium) from uncontaminated sediment (Serne et al. 2002a). In addition, the acid extract dissolved very little of the silicon, about $15 \mathrm{wt} \%$ of the aluminum and titanium, between about 40 to $60 \mathrm{wt} \%$ of the iron and manganese, and 25 to $70 \mathrm{wt} \%$ of the various trace metals. The dissolution efficiency for the sodium, silicon, barium, and trace metal oxides in samples from borehole 299-W23-19 is similar to that of the uncontaminated sediment. However, dissolution of magnesium, aluminum, potassium, calcium, manganese, and iron by acid digestion is greater for the samples from borehole 299-W23-19 than for the uncontaminated borehole samples. The higher dissolution percentages may be a factor of the extract preparation rather than greater amounts of tank liquor in the samples from borehole 299-W23-19. 


\subsubsection{Particle Size Measurements on Vadose Zone Sediment}

Both dry sieving and wet sieving/hydrometer methods were used to determine the particle-size distributions of several samples from borehole 299-W23-19. Dry sieving was used to help

Table 4.19 Percentage of Elements Extracted by 8 M Nitric Acid

\begin{tabular}{|c|c|c|c|c|c|c||}
\hline Element & 17DE & 19G & 26A & 31G & 38A & 39D \\
\hline $\mathrm{Na}$ & --- & --- & -- & -- & -- &.- \\
\hline $\mathrm{Mg}$ & 66.67 & 49.60 & 47.90 & 58.02 & 63.57 & 38.36 \\
\hline $\mathrm{Al}$ & 28.39 & 16.45 & 12.75 & 19.61 & 25.31 & 14.16 \\
\hline $\mathrm{Si}$ & 0.00 & 0.00 & 0.00 & 0.00 & 0.00 & 0.00 \\
\hline $\mathrm{K}$ & 15.29 & 9.25 & 7.35 & 10.05 & 15.21 & 10.76 \\
\hline $\mathrm{Ca}$ & 53.03 & 41.84 & 38.80 & 51.08 & 47.81 & 34.50 \\
\hline $\mathrm{Cr}$ & 47.04 & 55.13 & 50.83 & 40.08 & 39.50 & 32.72 \\
\hline $\mathrm{Mn}$ & 83.51 & 63.49 & 57.19 & 64.08 & 63.88 & 35.89 \\
\hline $\mathrm{Fe}$ & 71.19 & 55.83 & 54.64 & 64.26 & 71.71 & 39.77 \\
\hline $\mathrm{Co}$ & 15.38 & 13.29 & 15.29 & 20.00 & 26.56 & 15.25 \\
\hline $\mathrm{Ni}$ & 64.91 & 53.57 & 64.04 & 62.19 & 87.76 & 78.32 \\
\hline $\mathrm{Cu}$ & 60.75 & 55.29 & 60.38 & 60.22 & 84.44 & 60.34 \\
\hline $\mathrm{Zn}$ & 88.36 & 78.64 & 71.79 & 79.89 & 96.39 & 54.51 \\
\hline $\mathrm{As}$ & 65.38 & 102.56 & 55.13 & 50.00 & 67.92 & 50.36 \\
\hline $\mathrm{Se}$ & $>2.38$ & $>2.38$ & $>2.61$ & $>2.00$ & $>3.33$ & $>5.20$ \\
\hline $\mathrm{Sr}$ & 26.17 & 12.89 & 10.55 & 19.32 & 18.51 & 14.75 \\
\hline $\mathrm{Mo}$ & 3.46 & $>7.50$ & $>13.45$ & $>2.50$ & $>4.58$ & 19.22 \\
\hline $\mathrm{Ag}$ & 0.63 & 0.33 & 0.20 & 0.38 & 0.67 & 0.43 \\
\hline $\mathrm{Ba}$ & 25.59 & 13.83 & 8.80 & 12.82 & 23.39 & 15.60 \\
\hline $\mathrm{Pb}$ & 63.64 & 46.98 & 34.48 & 52.80 & 80.95 & 68.18 \\
\hline $\mathrm{U}$ & $>13.33$ & $>10.00$ & $>7.22$ & $>14.22$ & $>24.55$ & $>12.97$ \\
\hline
\end{tabular}

determine the geologic contacts between the Hanford formation $\mathrm{H} 1$ unit and the overlying H1a and underlying $\mathrm{H} 2$ units. Table 4.20 gives the results from the dry sieve analyses; Table 4.21 gives the Folk/Wentworth classification based on the results presented in Table 4.20. The data clearly show the geologic contacts between the relatively coarse-grained Hanford formation H1 unit and overlying and underlying, relatively fine-grained, Hanford formation $\mathrm{H} 1 \mathrm{a}$ and $\mathrm{H} 2$ units. The Hanford formation $\mathrm{H} 1$ unit upper contact is at 23.2 and 23.8 meters ( 76 to 78 feet bgs) and the lower contact is at 26.5 meters (87 feet bgs).

The particle-size distributions measured by the wet sieve/hydrometer method are shown in Table 4.22 (along with the dry sieve results for comparison) and Figures 4.8 to 4.11. For every sample from which both dry sieve and wet sieve data are available, the dry sieve particle-size distribution is biased toward a coarser-grain size distribution. This has also been noted from data from other boreholes at Waste 
Management Area S-SX (Serne et al. 2002a, Serne et al. 2002b) and results from the fact that the water in the wet sieve method dissociates silt and clay aggregates.

Figure 4.10 shows particle-size distributions from three samples of the upper Plio-Pleistocene unit. The data show that this unit grades into the coarser grained, underlying lower Plio-Pleistocene unit so that the precise location of the contact is difficult to choose based solely on the particle-size distributions.

Table 4.20 Dry Sieve Particle Size Distributions of Samples from Borehole 299-W23-19 (wt\%)

\begin{tabular}{|c|c|c|c|c|c|c|c|c|c|c|}
\hline \multirow[b]{4}{*}{$\begin{array}{c}\text { Sample } \\
\text { ID } \\
\end{array}$} & & \multicolumn{2}{|c|}{ Gravel } & \multicolumn{6}{|c|}{ Sand } & \multirow{3}{*}{$\begin{array}{c}\text { Mud } \\
\text { Silt \& } \\
\text { Clay } \\
\text { Pan }\end{array}$} \\
\hline & & \multirow{2}{*}{$\begin{array}{c}\begin{array}{c}\text { Fine } \\
\text { Pebble }\end{array} \\
5\end{array}$} & \multirow{2}{*}{$\begin{array}{c}\begin{array}{c}\text { Very } \\
\text { Fine } \\
\text { Pebble }\end{array} \\
10\end{array}$} & \multirow{2}{*}{$\begin{array}{c}\begin{array}{c}\text { Very } \\
\text { Coarse }\end{array} \\
18\end{array}$} & \multirow{2}{*}{$\begin{array}{c}\text { Coarse } \\
35\end{array}$} & \multirow{2}{*}{\begin{tabular}{|c|} 
Medium \\
60
\end{tabular}} & \multicolumn{2}{|c|}{ Fine } & \multirow{2}{*}{$\begin{array}{l}\text { Very } \\
\text { Fine } \\
230\end{array}$} & \\
\hline & Seive \# & & & & & & 70 & 120 & & \\
\hline & $\begin{array}{c}\text { Depth } \\
(\mathbf{f t})^{\mathrm{a}}\end{array}$ & $>4 \mathrm{~mm}$ & 2-4 mm & $1-2 \mathrm{~mm}$ & $\begin{array}{c}0.5-1.0 \\
\mathrm{~mm}\end{array}$ & \begin{tabular}{|c|}
$0.25-0.5$ \\
$\mathrm{~mm}$
\end{tabular} & $\begin{array}{c}0.21-0.25 \\
\mathrm{~mm}\end{array}$ & \begin{tabular}{|c|}
$0.125-$ \\
$0.21 \mathrm{~mm}$ \\
\end{tabular} & $\begin{array}{c}0.0625- \\
0.125 \\
\text { mm } \\
\end{array}$ & $\begin{array}{c}<0.0625 \\
\mathrm{~mm} \\
\end{array}$ \\
\hline $19 \mathrm{~J}$ & $72-72.5$ & 0.00 & 0.14 & 1.94 & 19.79 & 46.48 & 6.25 & 12.68 & 9.13 & 3.60 \\
\hline 19I & 72.5-73 & 1.50 & 0.65 & 5.39 & 32.79 & 40.70 & 4.10 & 8.04 & 4.28 & 2.54 \\
\hline $19 \mathrm{H}$ & $73-73.5$ & 1.38 & 0.61 & 5.45 & 34.81 & 32.68 & 3.22 & 8.27 & 8.08 & 5.49 \\
\hline $19 \mathrm{G}$ & $73.5-74$ & 0.00 & 0.30 & 1.01 & 3.88 & 10.14 & 4.60 & 29.76 & 32.32 & 17.98 \\
\hline $19 \mathrm{~F}$ & $74-74.5$ & 0.00 & 0.10 & 0.95 & 3.12 & 8.83 & 3.71 & 23.47 & 36.67 & 23.14 \\
\hline $19 \mathrm{E}$ & 74.5-75 & 0.00 & 0.01 & 0.55 & 2.66 & 13.15 & 4.08 & 23.82 & 34.82 & 20.90 \\
\hline 19D & $75-75.5$ & 0.00 & 0.25 & 1.43 & 7.87 & 28.16 & 6.40 & 25.33 & 20.33 & 10.22 \\
\hline $19 \mathrm{C}$ & $75.5-76$ & 0.43 & 2.17 & 9.80 & 34.37 & 36.75 & 2.86 & 5.92 & 4.16 & 3.55 \\
\hline 19B & $76-76.5$ & 0.00 & 0.92 & 6.60 & 41.05 & 35.33 & 2.47 & 5.24 & 4.38 & 4.00 \\
\hline $20 \mathrm{H}$ & 78-78.5 & 15.63 & 14.24 & 20.03 & 19.98 & 12.51 & 2.20 & 5.91 & 5.65 & 3.85 \\
\hline $20 \mathrm{G}$ & 78.5-79 & 0.51 & 1.26 & 7.57 & 15.32 & 28.85 & 8.02 & 19.35 & 12.19 & 6.93 \\
\hline $20 \mathrm{~F}$ & $79-79.5$ & 0.00 & 0.70 & 3.63 & 6.62 & 11.07 & 3.62 & 24.55 & 32.42 & 17.40 \\
\hline $20 \mathrm{E}$ & 79.5-80 & 0.00 & 2.37 & 4.13 & 5.06 & 5.74 & 3.05 & 29.25 & 37.05 & 13.36 \\
\hline $20 \mathrm{D}$ & 80-80.5 & 0.00 & 0.13 & 0.58 & 1.01 & 4.76 & 1.87 & 17.31 & 51.99 & 22.34 \\
\hline $21 \mathrm{E}$ & $81.5-82.5$ & 18.20 & 6.41 & 20.39 & 31.35 & 15.42 & 1.25 & 2.85 & 2.01 & 2.14 \\
\hline $21 \mathrm{D}$ & $82.5-83.5$ & 43.22 & 11.18 & 15.03 & 14.85 & 9.67 & 1.07 & 2.22 & 1.51 & 1.24 \\
\hline $21 \mathrm{C}$ & $83.5-84.5$ & 4.76 & 9.89 & 25.35 & 32.66 & 17.65 & 1.40 & 3.16 & 2.42 & 2.71 \\
\hline $21 \mathrm{~B}$ & $84.5-85.5$ & 7.07 & 13.74 & 33.04 & 29.62 & 9.03 & 0.86 & 2.14 & 1.94 & 2.55 \\
\hline $21 \mathrm{~A}$ & $85.5-86.5$ & 18.29 & 11.03 & 25.59 & 29.77 & 8.69 & 0.83 & 1.97 & 1.69 & 2.15 \\
\hline $22 \mathrm{E}$ & $86.6-87.6$ & 23.23 & 5.23 & 11.54 & 16.58 & 11.17 & 2.60 & 12.93 & 11.53 & 5.02 \\
\hline $22 \mathrm{D}$ & 87.6-88.6 & 0.00 & 0.11 & 0.10 & 0.56 & 2.91 & 1.53 & 18.83 & 56.16 & 19.80 \\
\hline $22 \mathrm{C}$ & 88.6-89.6 & 0.00 & 0.02 & 0.09 & 4.73 & 48.59 & 7.95 & 17.45 & 14.05 & 7.13 \\
\hline $22 \mathrm{~B}$ & $89.6-90.6$ & 0.00 & 0.03 & 0.06 & 0.90 & 21.81 & 10.58 & 33.20 & 23.65 & 9.76 \\
\hline
\end{tabular}




\begin{tabular}{|r|r|r|r|r|r|r|r|r|r|r||}
\hline 22A & $90.6-91.6$ & 0.00 & 0.00 & 0.05 & 0.33 & 32.22 & 17.29 & 28.38 & 13.94 & 7.78 \\
\hline 24C & $92.5-93.5$ & 2.62 & 0.73 & 3.29 & 5.76 & 14.05 & 5.42 & 25.39 & 33.81 & 8.93 \\
\hline 24B & $93.5-94.5$ & 0.00 & 0.45 & 1.40 & 4.94 & 16.67 & 6.31 & 26.73 & 32.33 & 11.17 \\
\hline 24A & $94.5-95.5$ & 0.00 & 0.00 & 0.01 & 0.29 & 1.02 & 0.72 & 12.41 & 63.23 & 22.32 \\
\hline 25E & $95.6-96.6$ & 13.78 & 0.22 & 0.21 & 2.74 & 25.02 & 7.23 & 21.97 & 21.85 & 6.79 \\
\hline 25D & $96.6-97.6$ & 0.00 & 0.00 & 0.11 & 1.74 & 21.65 & 9.14 & 29.44 & 30.87 & 7.04 \\
\hline 25C & $97.6-98.6$ & 0.00 & 0.01 & 0.10 & 1.53 & 22.71 & 6.25 & 27.34 & 34.64 & 7.43 \\
\hline 25B & $98.6-99.6$ & 0.00 & 0.02 & 0.24 & 1.20 & 21.75 & 7.02 & 29.32 & 32.58 & 7.88 \\
\hline & $99.6-$ & & & & & & & & & \\
25A & 100.6 & 0.00 & 0.04 & 1.18 & 2.60 & 14.90 & 5.24 & 22.88 & 33.08 & 20.09 \\
\hline
\end{tabular}

(a) multiply by 0.3048 to convert to meters

Table 4.21 Folk/Wentworth Classification of Sample from Borehole 299-W23-19 (wt\%).

\begin{tabular}{|c|c|c|c|c|c|c|c|}
\hline $\begin{array}{l}\text { Sample } \\
\text { ID }\end{array}$ & $\begin{array}{c}\text { Depth } \\
(\mathbf{f t} \text { bgs) })^{\text {a }} \\
\end{array}$ & Gravel & Sand & Mud & $\begin{array}{c}\text { Sand:Mud } \\
\text { Ratio } \\
\end{array}$ & CLASS $^{1}$ & Unit \\
\hline $19 \mathrm{~J}$ & $72-72.5$ & 0.14 & 96.27 & 3.60 & 26.8 & $S$ & $\mathrm{H} 1 \mathrm{a}$ \\
\hline 19I & $72.5-73$ & 2.15 & 95.31 & 2.54 & 37.5 & $S$ & $\mathrm{H} 1 \mathrm{a}$ \\
\hline $19 \mathrm{H}$ & 73-73.5 & 1.99 & 92.52 & 5.49 & 16.8 & $S$ & $\mathrm{H} 1 \mathrm{a}$ \\
\hline $19 \mathrm{G}$ & $73.5-74$ & 0.30 & 81.72 & 17.98 & 4.5 & (m)S & $\mathrm{H} 1 \mathrm{a}$ \\
\hline $19 \mathrm{~F}$ & 74-74.5 & 0.10 & 76.76 & 23.14 & 3.3 & $\mathrm{mS}$ & $\mathrm{H} 1 \mathrm{a}$ \\
\hline $19 \mathrm{E}$ & $74.5-75$ & 0.01 & 79.09 & 20.90 & 3.8 & $\mathrm{mS}$ & H1a \\
\hline $19 \mathrm{D}$ & $75-75.5$ & 0.25 & 89.52 & 10.22 & 8.8 & (m)S & $\mathrm{H} 1 \mathrm{a}$ \\
\hline $19 \mathrm{C}$ & $75.5-76$ & 2.60 & 93.85 & 3.55 & 26.5 & $\mathrm{~S}$ & H1a \\
\hline 19B & 76-76.5 & 0.92 & 95.07 & 4.00 & 23.8 & $S$ & $\mathrm{H} 1 \mathrm{a}$ \\
\hline $20 \mathrm{H}$ & 78-78.5 & 29.88 & 66.28 & 3.85 & 17.2 & $\mathrm{gS}$ & $\mathrm{H} 1$ \\
\hline $20 \mathrm{G}$ & 78.5-79 & 1.77 & 91.31 & 6.93 & 13.2 & $\mathrm{~S}$ & $\mathrm{H} 1$ \\
\hline $20 \mathrm{~F}$ & $79-79.5$ & 0.70 & 81.90 & 17.40 & 4.7 & $(\mathrm{~m}) \mathrm{S}$ & $\mathrm{H} 1$ \\
\hline $20 \mathrm{E}$ & $79.5-80$ & 2.37 & 84.27 & 13.36 & 6.3 & $(\mathrm{~m}) \mathrm{S}$ & $\mathrm{H} 1$ \\
\hline $20 \mathrm{D}$ & $80-80.5$ & 0.13 & 77.53 & 22.34 & 3.5 & $\mathrm{mS}$ & $\mathrm{H} 1$ \\
\hline $21 \mathrm{E}$ & $81.5-82.5$ & 24.61 & 73.25 & 2.14 & 34.2 & $\mathrm{gS}$ & $\mathrm{H} 1$ \\
\hline $21 \mathrm{D}$ & $82.5-83.5$ & 54.40 & 44.36 & 1.24 & 35.8 & sG & $\mathrm{H} 1$ \\
\hline $21 \mathrm{C}$ & $83.5-84.5$ & 14.65 & 82.64 & 2.71 & 30.5 & $\mathrm{gS}$ & $\mathrm{H} 1$ \\
\hline $21 \mathrm{~B}$ & $84.5-85.5$ & 20.82 & 76.63 & 2.55 & 30.0 & $\mathrm{gS}$ & $\mathrm{H} 1$ \\
\hline $21 \mathrm{~A}$ & $85.5-86.5$ & 29.32 & 68.53 & 2.15 & 31.9 & $\mathrm{gS}$ & $\mathrm{H} 1$ \\
\hline $22 \mathrm{E}$ & 86.6-87.6 & 28.47 & 66.35 & 5.02 & 13.2 & $\mathrm{gS}$ & $\mathrm{H} 1$ \\
\hline $22 \mathrm{D}$ & 87.6-88.6 & 0.11 & 80.08 & 19.80 & 4.0 & $(\mathrm{~m}) \mathrm{S}$ & $\mathrm{H} 1$ \\
\hline $22 \mathrm{C}$ & $88.6-89.6$ & 0.02 & 92.86 & 7.13 & 13.0 & $S$ & $\mathrm{H} 1$ \\
\hline
\end{tabular}




\begin{tabular}{|c|c|c|c|c|c|c|c|}
\hline $22 \mathrm{~B}$ & 89.6-90.6 & 0.03 & 90.21 & 9.76 & 9.2 & $S$ & $\mathrm{H} 1$ \\
\hline $22 \mathrm{~A}$ & $90.6-91.6$ & 0.00 & 92.22 & 7.78 & 11.9 & $S$ & $\mathrm{H} 1$ \\
\hline $24 \mathrm{C}$ & $92.5-93.5$ & 3.35 & 87.72 & 8.93 & 9.8 & $S$ & $\mathrm{H} 1$ \\
\hline $24 \mathrm{~B}$ & $93.5-94.5$ & 0.45 & 88.38 & 11.17 & 7.9 & $(\mathrm{~m}) \mathrm{S}$ & $\mathrm{H} 2$ \\
\hline $24 \mathrm{~A}$ & $94.5-95.5$ & 0.00 & 77.68 & 22.32 & 3.5 & $\mathrm{mS}$ & $\mathrm{H} 2$ \\
\hline $25 \mathrm{E}$ & $95.6-96.6$ & 13.99 & 79.03 & 6.79 & 11.6 & $\mathrm{gS}$ & $\mathrm{H} 2$ \\
\hline $25 \mathrm{D}$ & 96.6-97.6 & 0.00 & 92.96 & 7.04 & 13.2 & $S$ & $\mathrm{H} 2$ \\
\hline $25 \mathrm{C}$ & 97.6-98.6 & 0.01 & 92.56 & 7.43 & 12.5 & $S$ & $\mathrm{H} 2$ \\
\hline $25 \mathrm{~B}$ & 98.6-99.6 & 0.02 & 92.10 & 7.88 & 11.7 & $S$ & $\mathrm{H} 2$ \\
\hline $25 \mathrm{~A}$ & 99.6-100.6 & 0.04 & 79.87 & 20.09 & 4.0 & $(\mathrm{~m}) \mathrm{S}$ & $\mathrm{H} 2$ \\
\hline
\end{tabular}

Table 4.22 Comparison of Particle Size Distribution [wt.] Measured by the Wet Sieve/Hydrometer Method and the Dry Sieve Method

\begin{tabular}{|c|c|c|c|c|c|c|c|}
\hline $\begin{array}{l}\text { Sample } \\
\text { ID }\end{array}$ & $\begin{array}{c}\text { Depth } \\
(\mathbf{f t} \text { bgs })^{\mathrm{a}}\end{array}$ & Unit & Type & Wt\% Gravel & Wt\% Sand & Wt\% Silt & Wt\% Clay \\
\hline $17 \mathrm{DE}$ & 64.75 & $\mathrm{H} 1 \mathrm{a}$ & Wet & 0.15 & 48.31 & 47.24 & 4.30 \\
\hline $19 \mathrm{~J}$ & $72-72.5$ & $\mathrm{H} 1 \mathrm{a}$ & Dry & 0.14 & 96.27 & \multicolumn{2}{|c|}{3.60} \\
\hline 19I & $72.5-73$ & $\mathrm{H} 1 \mathrm{a}$ & Dry & 2.15 & 95.31 & \multicolumn{2}{|c|}{2.54} \\
\hline $19 \mathrm{H}$ & $73-73.5$ & $\mathrm{H} 1 \mathrm{a}$ & Dry & 1.99 & 92.52 & \multicolumn{2}{|c|}{5.49} \\
\hline $19 \mathrm{G}$ & 73.5-74 & $\mathrm{H} 1 \mathrm{a}$ & Dry & 0.30 & 81.72 & \multicolumn{2}{|c|}{17.98} \\
\hline $19 \mathrm{G}$ & 73.75 & $\mathrm{H} 1 \mathrm{a}$ & Wet & 0.18 & 73.65 & 22.87 & 3.30 \\
\hline $19 \mathrm{~F}$ & $74-74.5$ & $\mathrm{H} 1 \mathrm{a}$ & Dry & 0.10 & 76.76 & \multicolumn{2}{|c|}{23.14} \\
\hline $19 \mathrm{E}$ & 74.5-75 & $\mathrm{H} 1 \mathrm{a}$ & Dry & 0.01 & 79.09 & \multicolumn{2}{|c|}{20.90} \\
\hline 19D & $75-75.5$ & $\mathrm{H} 1 \mathrm{a}$ & Dry & 0.25 & 89.52 & \multicolumn{2}{|c|}{10.22} \\
\hline 21D & $82.5-83.5$ & $\mathrm{H} 1$ & Dry & 54.40 & 44.36 & \multicolumn{2}{|c|}{1.24} \\
\hline $21 \mathrm{C}$ & $83.5-84.5$ & $\mathrm{H} 1$ & Dry & 14.65 & 82.64 & \multicolumn{2}{|c|}{2.71} \\
\hline $21 \mathrm{~B}$ & $84.5-85.5$ & $\mathrm{H} 1$ & Dry & 20.82 & 76.63 & \multicolumn{2}{|c|}{2.55} \\
\hline $21 \mathrm{~A}$ & $85.5-86.5$ & $\mathrm{H} 1$ & Dry & 29.32 & 68.53 & \multicolumn{2}{|c|}{2.15} \\
\hline $22 \mathrm{E}$ & 86.6-87.6 & $\mathrm{H} 1$ & Dry & 28.47 & 66.35 & \multicolumn{2}{|c|}{5.02} \\
\hline $22 \mathrm{D}$ & 87.6-88.6 & $\mathrm{H} 1$ & Dry & 0.11 & 80.08 & \multicolumn{2}{|c|}{19.80} \\
\hline $22 \mathrm{D}$ & 88.1 & $\mathrm{H} 1$ & Wet & 0.00 & 72.66 & 24.25 & 3.09 \\
\hline $22 \mathrm{C}$ & 88.6-89.6 & $\mathrm{H} 1$ & Dry & 0.02 & 92.86 & \multicolumn{2}{|c|}{7.13} \\
\hline $22 \mathrm{~B}$ & $89.6-90.6$ & $\mathrm{H} 1$ & Dry & 0.03 & 90.21 & \multicolumn{2}{|c|}{9.76} \\
\hline $22 \mathrm{~A}$ & $90.6-91.6$ & $\mathrm{H} 1$ & Dry & 0.00 & 92.22 & \multicolumn{2}{|c|}{7.78} \\
\hline $24 \mathrm{C}$ & $92.5-93.5$ & $\mathrm{H} 1$ & Dry & 3.35 & 87.72 & \multicolumn{2}{|c|}{8.93} \\
\hline
\end{tabular}




\begin{tabular}{|c|c|c|c|c|c|c|c|}
\hline $24 \mathrm{~B}$ & 93.5-94.5 & $\mathrm{H} 2$ & Dry & 0.45 & 88.38 & \multicolumn{2}{|c|}{11.17} \\
\hline $24 \mathrm{~A}$ & $94.5-95.5$ & $\mathrm{H} 2$ & Dry & 0.00 & 77.68 & \multicolumn{2}{|c|}{22.32} \\
\hline $25 \mathrm{E}$ & $95.6-96.6$ & $\mathrm{H} 2$ & Dry & 13.99 & 79.03 & \multicolumn{2}{|c|}{6.79} \\
\hline $25 \mathrm{D}$ & $96.6-97.6$ & $\mathrm{H} 2$ & Dry & 0.00 & 92.96 & \multicolumn{2}{|c|}{7.04} \\
\hline $25 \mathrm{C}$ & 97.6-98.6 & $\mathrm{H} 2$ & Dry & 0.01 & 92.56 & \multicolumn{2}{|c|}{7.43} \\
\hline $25 \mathrm{~B}$ & 98.6-99.6 & $\mathrm{H} 2$ & Dry & 0.02 & 92.10 & \multicolumn{2}{|c|}{7.88} \\
\hline $25 \mathrm{~A}$ & 99.6-100.6 & $\mathrm{H} 2$ & Dry & 0.04 & 79.87 & \multicolumn{2}{|c|}{20.09} \\
\hline $26 \mathrm{~A}$ & 104.95 & $\mathrm{H} 2$ & Wet & 0.09 & 85.45 & 11.37 & 3.09 \\
\hline $31 \mathrm{G}$ & 129.15 & $\mathrm{PPlz}$ & Wet & 0.00 & 13.21 & 79.29 & 7.50 \\
\hline $38 \mathrm{~A}$ & 155.1 & $\mathrm{PPlz}$ & Wet & 0.00 & 17.40 & 76.49 & 6.11 \\
\hline $39 \mathrm{D}$ & 155.35 & $\mathrm{PPlz}$ & Wet & 16.33 & 50.18 & 28.32 & 5.17 \\
\hline $43 \mathrm{~B}$ & 204.5 & Rwi(e) & Wet & 54.47 & 33.82 & 8.21 & 3.50 \\
\hline
\end{tabular}

(a) multiply by 0.3048 to convert to meters

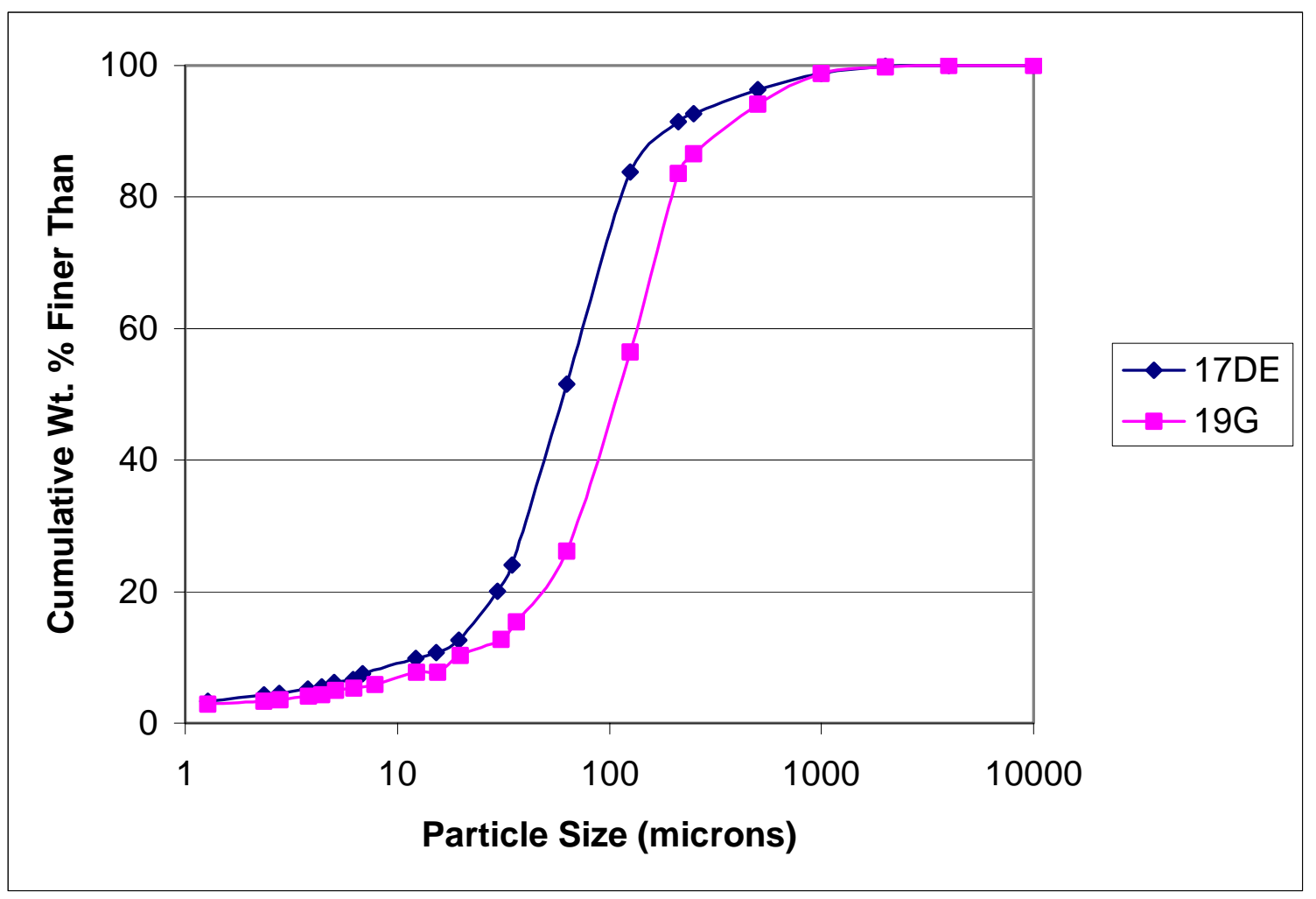

Figure 4.8 Particle-Size Distribution of Samples from the Hanford Formation H1a Unit in Borehole 299W23-19 


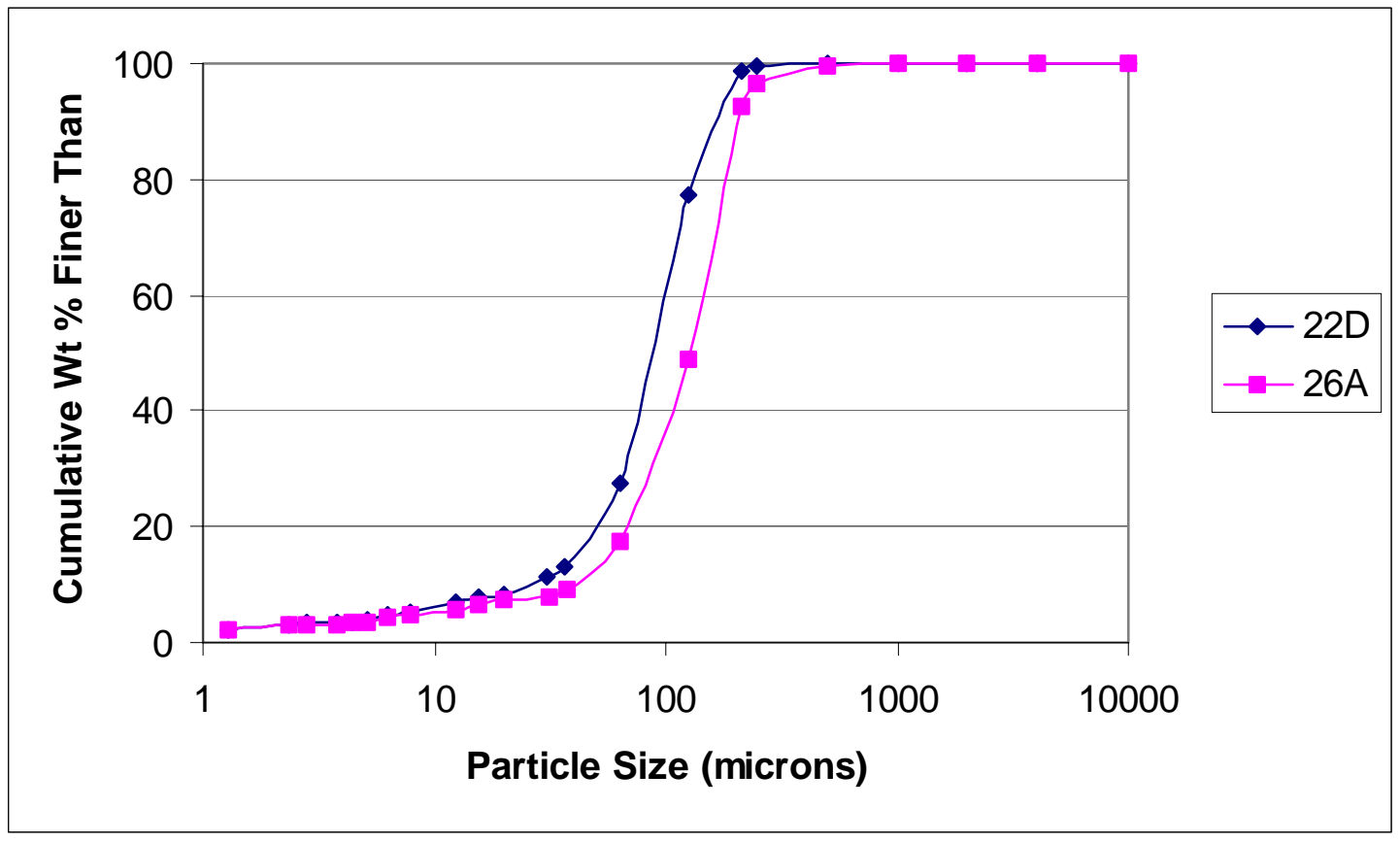

Figure 4.9 Particle-Size Distribution of Samples from the Hanford Formation H1 and H2 Units in Borehole 299-W23-19

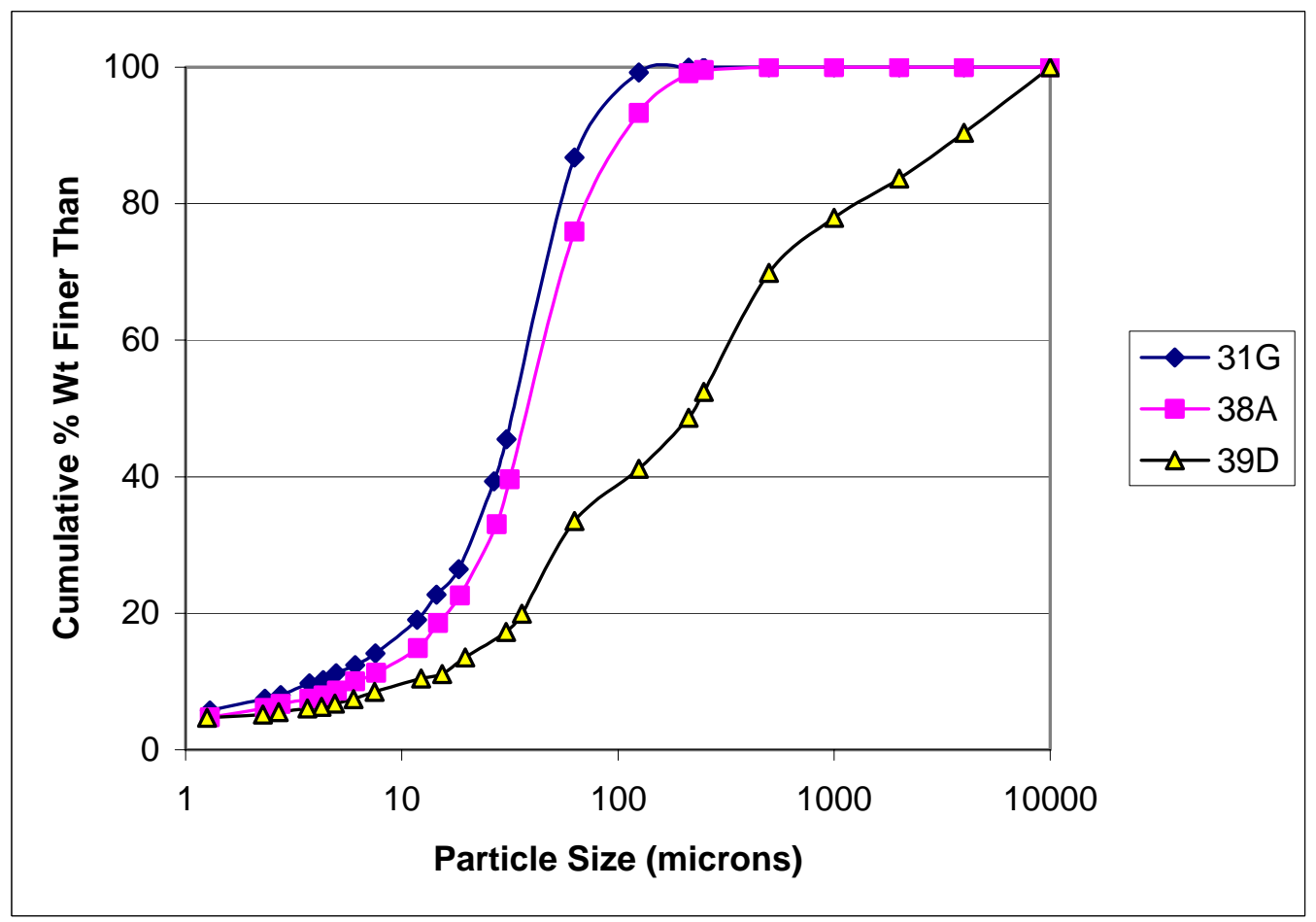

Figure 4.10 Particle-Size Distribution of Samples from the Upper Plio-Pleistocene Unit in Borehole 299W23-19 


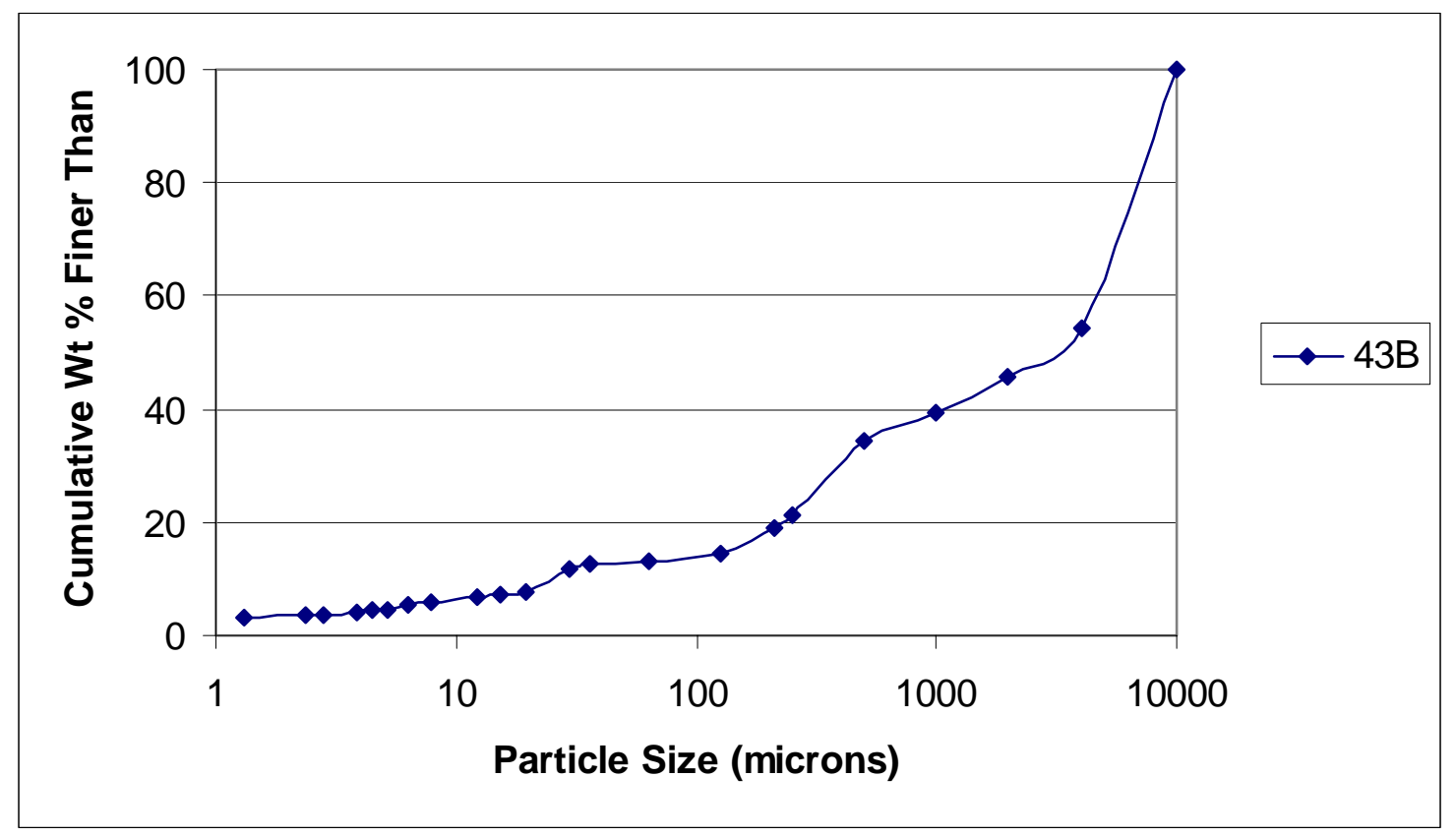

Figure 4.11 Particle Size Distribution of a Sample of Ringold Formation, Member of Wooded Island Unit E from Borehole 299-W23-19

\subsubsection{Particle Density of Bulk Sediment Samples}

The particle density of selected samples from borehole 299-W23-19 is shown in Table 4.23. The data in the table are similar to those of uncontaminated sediment from the same lithologic units (Serne et al. 2002a) in nearby boreholes.

Table 4.23 Particle Density of Bulk Sediment from 299-W23-19 Borehole

\begin{tabular}{|c|c|c|c|}
\hline $\begin{array}{c}\text { Sample } \\
\text { ID }\end{array}$ & $\begin{array}{c}\text { Depth } \\
\text { (ft bgs) }^{\mathbf{a}}\end{array}$ & Geologic Unit & Particle Density (g/cc) \\
\hline \hline $17 \mathrm{DE}$ & 64.75 & $\mathrm{H} 1 \mathrm{a}$ & 2.716 \\
\hline $19 \mathrm{G}$ & 73.75 & $\mathrm{H} 1 \mathrm{a}$ & 2.706 \\
\hline $22 \mathrm{D}$ & 88.10 & $\mathrm{H} 1$ & 2.708 \\
\hline $26 \mathrm{~A}$ & 104.95 & $\mathrm{H} 2$ & 2.712 \\
\hline $31 \mathrm{G}$ & 129.15 & $\mathrm{PPlz}$ & 2.731 \\
\hline $38 \mathrm{~A}$ & 155.10 & $\mathrm{PPlz}$ & 2.756 \\
\hline $39 \mathrm{D}$ & 155.35 & $\mathrm{PPlz}$ & 2.863 \\
\hline 43B & 204.50 & $\mathrm{Rwi}(\mathrm{e})$ & 2.680 \\
\hline
\end{tabular}

(a) multiply 0.3048 to convert to meters 


\subsubsection{Mineralogy}

Semiquantitative results of $\mathrm{x}$-ray diffraction analyses of the bulk samples from eight depths in borehole 299-W23-19 are given in Table 4.24. The data indicate that the sediment is mostly quartz $(\sim 40$ to 55\%) and feldspar ( 25 to $65 \%$ ), with lesser amounts of mica, chlorite, and smectite. Plagioclase feldspar is 2 to 6 times more abundant than potassium feldspar, with the exception of sample 22D (26.7 to 27 meters [87.6 to 88.6 feet bgs]), which has more potassium feldspar than plagioclase feldspar. Minor amounts of amphibole and calcite also were detected in the sediment. Example x-ray diffraction patterns are shown in Appendix A.

The silt and clay fractions of selected samples were separated by wet sieving the bulk sample and then analyzed by x-ray diffraction. Results for the silt fraction are similar to those of the bulk sediment; that is, quartz, K-feldspar, and plagioclase dominate the samples. Minor amounts of mica, chlorite, and amphibole also are present in lesser amounts. Additionally, in sample 31G (39.3 to 39.4 meters [128.9 to 129.4 feet]) reflections indicative of dolomite were observed.

The clay fraction $(<2 \mu \mathrm{m})$ is dominated by four clay minerals: illite, smectite, chlorite, and kaolinite. Minor amounts of quartz, feldspar, and amphibole also are present. The smectites, when saturated with $\mathrm{Mg}^{2+}$, give a 001 reflection of $15.0 \AA$ which overlaps with the chlorite 001 reflection at $14.1 \AA$. After ethylene glycol solvation, the smectite 001 reflection expands to $17 \AA$, leaving the chlorite 001 (14.1 $\mathrm{A}$ ) and illite $001(10 \AA)$ reflections unchanged. Saturating the sample with $\mathrm{K}^{+}$shifts smectite 001 reflection to approximately $12 \AA$ again, leaving the chlorite and illite 001 reflections unaffected. Heating the $\mathrm{K}^{+}$saturated sample to $575^{\circ} \mathrm{C}$ results in collapse of the smectite interlayer from $12.0 \AA$ to $10.0 \AA$ and the kaolinite reflections at 7.01 $\AA$ and $3.58 \AA$ disappear. The chlorite reflection at $14.1 \AA$ remains unchanged, whereas the chlorite $7.1 \AA$ and $3.54 \AA$ reflections disappear.

Table 4.24. Semiquantitative Mineral Composition for Bulk Samples (wt\%)

\begin{tabular}{|l|c|c|c|c|c|c|c||}
\hline \multicolumn{1}{|c|}{ Sample ID } & $\begin{array}{c}\text { Depth } \\
(\mathbf{f t} \text { bgs) }\end{array}$ & $\begin{array}{c}\text { Geologic } \\
\text { Unit }\end{array}$ & Quartz & K-Feldspar & Plagioclase & Calcite & Total \\
\hline \hline 17DE & $64.0-64.5$ & H1a & 40 & 5 & 30 & 10 & 85 \\
\hline 19G & $73.5-74.0$ & H1a & 55 & 20 & 30 & 5 & 110 \\
\hline 22D & $87.6-88.6$ & H1 & 40 & 25 & 15 & $<5$ & 85 \\
\hline 26A & $104.7-105.2$ & H2 & 50 & 10 & 30 & $<5$ & 95 \\
\hline 31G & $128.9-129.4$ & $\mathrm{PPlz}$ & 50 & 5 & 30 & $<5$ & 90 \\
\hline 38A & $154.85-155.35$ & $\mathrm{PPlz}$ & 50 & 5 & 20 & 10 & 85 \\
\hline 39D & $155.1-155.6$ & $\mathrm{PPlz}$ & 40 & 15 & 50 & $<5$ & 110 \\
\hline 43B & $204.1-204.9$ & Rwi(e) & 45 & 10 & 40 & $<5$ & 95 \\
\hline
\end{tabular}

(a) multiply by 0.3048 to convert to meters

The results of semiquantitative mineral analysis of the clay fractions are given in Table 4.25. Overall, smectite, illite, chlorite, and kaolinite are the dominant minerals in the clay fraction, totaling 45 to $95 \mathrm{wt} \%$ 
Table 4.25. Semiquantitative Analysis of the Clay Fraction of Samples from Borehole 299-W23-19 $(\mathrm{wt} \%)$

\begin{tabular}{||l|c|c|c|c|c|c|c|c|c||}
\hline \hline Sample ID & $\begin{array}{c}\text { Depth } \\
(\mathbf{f t} \text { bgs })^{\mathbf{a}}\end{array}$ & Geol.Unit & Quartz & Feld. & Smectite & Illite & Chlorite & Kaolinite & Total \\
\hline \hline 17DE & $64.0-64.5$ & H1a & 5 & 5 & 20 & 15 & 10 & 5 & 55 \\
\hline 19G & $73.5-74.0$ & H1a & 5 & 5 & 20 & 25 & 20 & 5 & 80 \\
\hline 22D & $87.6-88.6$ & H1 & 5 & 5 & 20 & 30 & 30 & 10 & 100 \\
\hline 26A & $104.7-105.2$ & H2 & 10 & 5 & 30 & 30 & 30 & 10 & 115 \\
\hline 31G & $128.9-129.4$ & PPlz & 10 & 5 & 30 & 25 & 25 & 10 & 105 \\
\hline 38A & $154.9-155.4$ & PPlz & 5 & 5 & 30 & 20 & 20 & 5 & 85 \\
\hline 39D & $155.1-155.6$ & PPlz & 5 & 5 & 30 & 20 & 25 & 5 & 80 \\
\hline 43B & $204.1-204.9$ & Rwi(e) & 10 & 5 & 35 & 10 & 20 & 5 & 85 \\
\hline
\end{tabular}

(a) multiply by 0.3048 to convert to meters

Total weight percent of the clay fractions ranges from a low of $55 \%$ to a high of $100 \%$. Smectites, range in abundance from as high as $35 \mathrm{wt} \%$ (sample 43B from 204.1 to 204.9 foot depth) to as low as 20 $\mathrm{wt} \%$; illite makes up between about 10 to $30 \mathrm{wt} \%$. Chlorite concentrations range from a low of $10 \mathrm{wt} \%$ (samples 17DE at 19.5 meters [64 feet bgs]) to a high of $30 \mathrm{wt} \%$ (22D at 26.7 to 27 meters [87.6 to 88.6 feet] and 26A at 31.9 to 32 meters [104.7 to 105.2 feet bgs]). Minor amounts of kaolinite $(<10 \mathrm{wt} \%)$ were detected at all depths. Quartz and feldspar make up about 5 to $15 \mathrm{wt}$ of the clay-size fraction. Minor amounts of amphibole also were identified in the clay fraction, however amphibole was not quantified.

X-ray fluorescence analysis on the $<2 \mu \mathrm{m}$ fraction was conducted on each sample for which x-ray diffraction analysis was done. The x-ray fluorescence results, expressed as weight percent oxides, are presented in Table 4.18. Overall, approximately 50 to $60 \mathrm{wt} \%$ of the clay fraction consists of silica, followed by lesser amounts of aluminum oxide (about10 to18 wt \%) and ferric oxide (about 9 to $12 \mathrm{wt} \%$ ). The clay fraction contains between 4.9 and $13.0 \%$ (most samples are less than 9.5\%) of the total ferric oxide in the bulk sediment. Iron oxides were detected in trace amounts during the transmission electron microscopy (TEM) analysis of the clay fraction, which suggests that most of the iron in the $<2 \mu \mathrm{m}$ fraction is incorporated in the clay minerals and amphibole.

The abundance of illite can be estimated from the x-ray fluorescence data from the clay fraction. The amount of potassium from the $\mathrm{x}$-ray fluorescence analyses was converted to the equivalent amount of illite using an ideal illite formula with $0.61 \mathrm{~K}^{+}$per $\mathrm{O}_{10}(\mathrm{OH})_{2}$ (Reynolds and Moore 1989) and assuming all potassium is in the illite. The abundances of illite in each sample (from shallowest to deepest) were $12,20,25,27,22,20,20$, and $18 \mathrm{wt} \%$. The illite abundance determined by $\mathrm{x}$-ray diffraction for sample $43 \mathrm{~B}$ is about $50 \%$ low relative to the $\mathrm{x}$-ray fluorescence results; otherwise, the amounts of illite estimated from the x-ray diffraction and the x-ray fluorescence analyses are in good agreement.

TEM images of illite from samples 22D, 31G, and 43B show large, angular, platy particles typical of weathered micas. The illite particles had distinct, sharp edges and were the largest sized particles in the clay fractions. Figure 4.12 is a typical illite from sample $31 \mathrm{G}$. 
Chemical analyses of the illites showed little variation in the silicon, aluminum, and potassium contents; however, large differences were noted in the iron (III) and magnesium contents. the TEM derived composition of about 20 illite particles and assuming all iron as iron (III), the following structural formula was calculated:

$$
\left[\mathrm{K}^{+}{ }_{0.73} \mathrm{Ca}_{0.02}\right]^{+0.77}\left[\left(\mathrm{Al}^{3+}{ }_{1.82} \mathrm{Mg}^{2+}{ }_{0.21} \mathrm{Fe}^{3+}{ }_{0.21} \mathrm{Ti}^{4+}{ }_{0.02}\right)^{-0.01}\left(\mathrm{Si}^{4+}{ }_{3.22} \mathrm{Al}^{3+}{ }_{0.78}\right)^{-0.78}\right]^{-0.79} \mathrm{O}_{10}(\mathrm{OH})_{2}
$$

Most of the layer charge for the above illite formula originates in the tetrahedral sheet (-0.78 eq/ $\left.\mathrm{O}_{10}(\mathrm{OH})_{2}\right)$, with very little contribution from the octahedral sheet $\left(-0.01 \mathrm{eq} / \mathrm{O}_{10 /(\mathrm{OH}) 2}\right)$. The total charge on the 2:1 layer is -0.79 eq per $\mathrm{O}_{10}(\mathrm{OH})_{2}$. The interlayer charge of +0.77 eq per $\mathrm{O}_{10}(\mathrm{OH})_{2}$ balances the negative charge from the 2:1 layer. The layer charge derived from the above formula is typical of illite. The iron content of the 20 illites examined by TEM ranges from 0.05 to 0.82 atoms per $\mathrm{O}_{10}(\mathrm{OH})_{2}$ and magnesium ranges between 0.02 and 0.78 atoms per $\mathrm{O}_{10}(\mathrm{OH})_{2}$. Traces of titanium were detected in most of the illites.

Chlorite particles in the clay fractions from samples 22D, 31G, and 43B have a similar, thin and platy morphology as illite in TEM images. Determinations of chlorite composition by TEM showed a significant variability in the concentrations of magnesium (II) and iron (II). The chemical compositions of the chlorites suggest that the mineral lies between the magnesium-rich chamosite and iron-rich chlinoclore solid solution members.

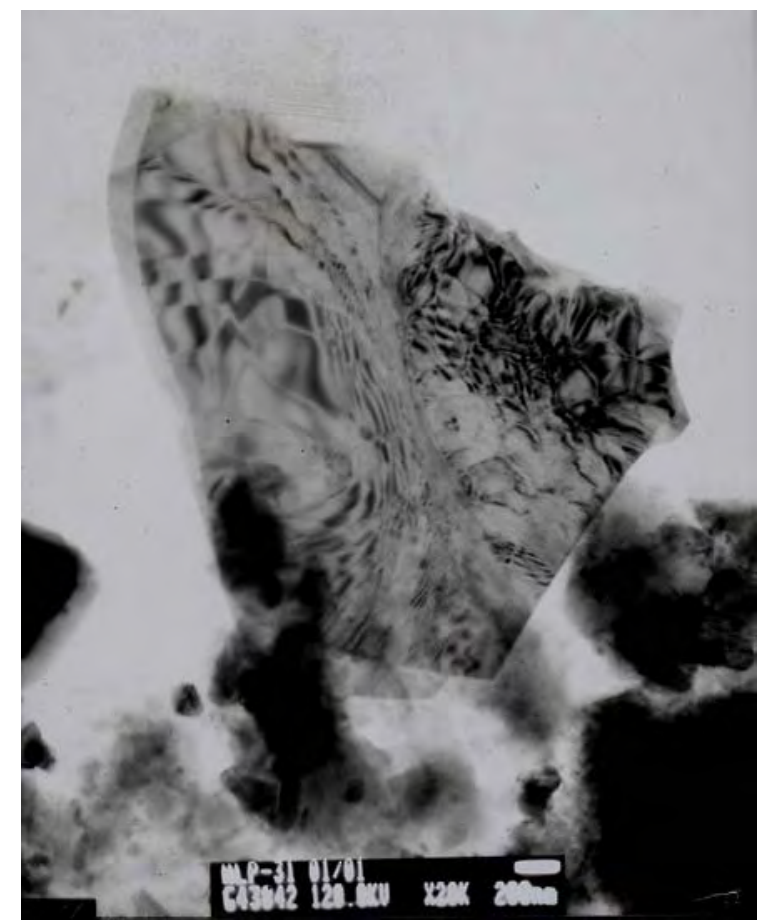

Figure 4.12. Typical Illite Particle from Sample 31G. The illite particle is about $2 \mu \mathrm{m}$ across.

A structural formula for chlorites derived from an average of 15 TEM analyses yielded a formula that was unreasonable in terms of octahedral occupancy and layer charge. This probably results from small 
amounts of impurities included in the chemical composition of chlorite and the difficulty of partitioning octahedral cations between the two octahedral sheets.

Large flaky aggregates of the smaller smectite particles were easily distinguished from the other clay minerals (Figure 4.13). The aggregates tended to project thin films of smectite out from the dense center of the aggregates which incorporate some illite and iron oxides. A typical smectite aggregate exhibiting thin films from sample 43B is shown in Figure 4.14. The following structural formula was developed from the average TEM data collected on 24 smectites (samples 22D, 31G, and 43B):

$$
\left[\mathrm{K}_{0.09}^{+} \mathrm{Ca}_{0.02}\right]^{+0.13}\left[\left(\mathrm{Al}^{3+}{ }_{1.02} \mathrm{Mg}^{2+}{ }_{0.42} \mathrm{Fe}^{3+}{ }_{0.73} \mathrm{Ti}^{4+}{ }_{0.03}\right)^{+0.21}\left(\mathrm{Si}^{4+}{ }_{3.66} \mathrm{Al}^{3+}{ }_{0.34}\right)^{-0.34}\right]^{-0.13} \mathrm{O}_{10}(\mathrm{OH})_{2}
$$

The tetrahedral sheet has negative charge resulting from substitution of aluminum (III) for silicon (IV) This negative charge is balanced by the $+0.21 \mathrm{eq} / \mathrm{O}_{10}(\mathrm{OH})_{2}$ charge on the octahedral sheet and by the interlayer cation charge of $+0.13 \mathrm{eq} / \mathrm{O}_{10}(\mathrm{OH})_{2}$. Iron content in the octahedral sites of all analyzed smectites ranged from 0.38 to 1.57 atoms per $\mathrm{O}_{10}(\mathrm{OH})_{2}$. The interlayer cation, potassium, seemed to be more concentrated in smectites from sample $31 \mathrm{G}$ as compared to samples $22 \mathrm{D}$ and $44 \mathrm{~B}$, even though each sample was saturated with the calcium chloride prior to the analysis. One possible explanation would be the presence of minor amounts of illite/smectite mixed layering, especially in sample $31 \mathrm{G}$. Potassium incorporated in the illite/smectite mixed layer mineral would not be exchangeable with other cations. Trace amounts of titanium were also detected in most smectites examined.

The above structural formula for smectite is anomalous in that it has a very low interlayer charge, a high octahedral charge, and a large octahedral occupancy. These anomalies could result from impurities in the analyzed sample and misappropriation of octahedral cations.

Kaolinite particles were platy in habit (Figure 4.15), and signs of weathering were common. The particles appeared to be composed of many smaller platelets with rough edges. Analysis of several kaolinites from each depth showed a slight substitution of iron in the octahedral sites for aluminum, as shown in the structural formula:

$$
\left(\mathrm{Al}_{3.90} \mathrm{Fe}_{0.09}\right) \mathrm{Si}_{4.00} \mathrm{O}_{10}(\mathrm{OH})_{8}
$$

Additionally, minerals such as sepiolite, apatite, iron oxide, sodium plagioclase, amphibole, and anatase possibly were detected in trace amounts during TEM analysis.

Mineral assemblages, concentrations, and structural formulas from samples of borehole 299-W23-19 were similar to data reported earlier on uncontaminated sediment from nearby boreholes (Serne et al. 2002a). No evidence of caustic tank liquor reacting with the sediment and altering the mineral phases or their properties was observed during the mineral studies on samples from borehole 299-W23-19. 


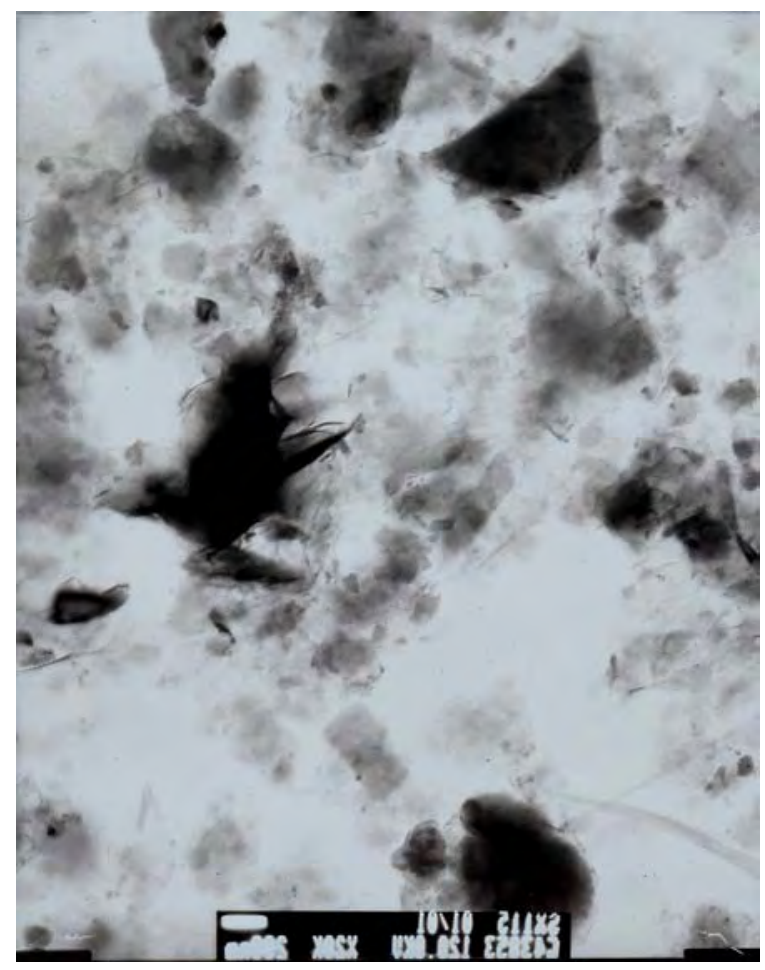

Figure 4.13. Image of Clay Particles in Sample 31G. The dense smectite aggregate (middle, left) is easily distinguished from the other clay minerals. The aggregrate is about $1.5 \mu \mathrm{m}$ across.

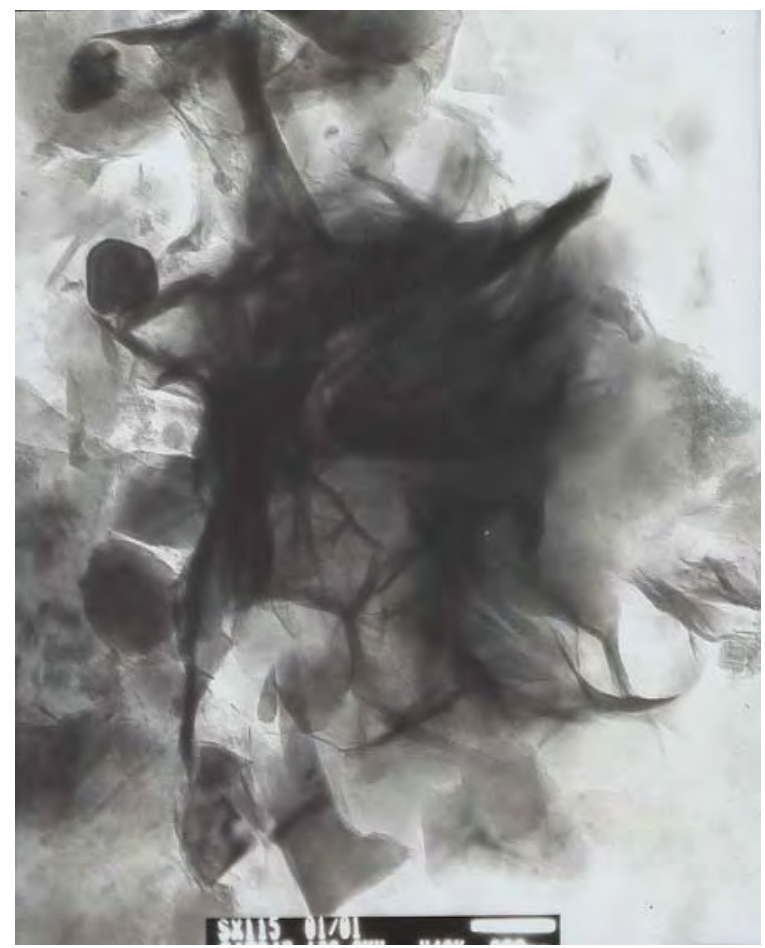

Figure 4.14. Large Smectite Aggregate from Sample 43B. Photo is about $1.5 \mu \mathrm{m}$ across. 


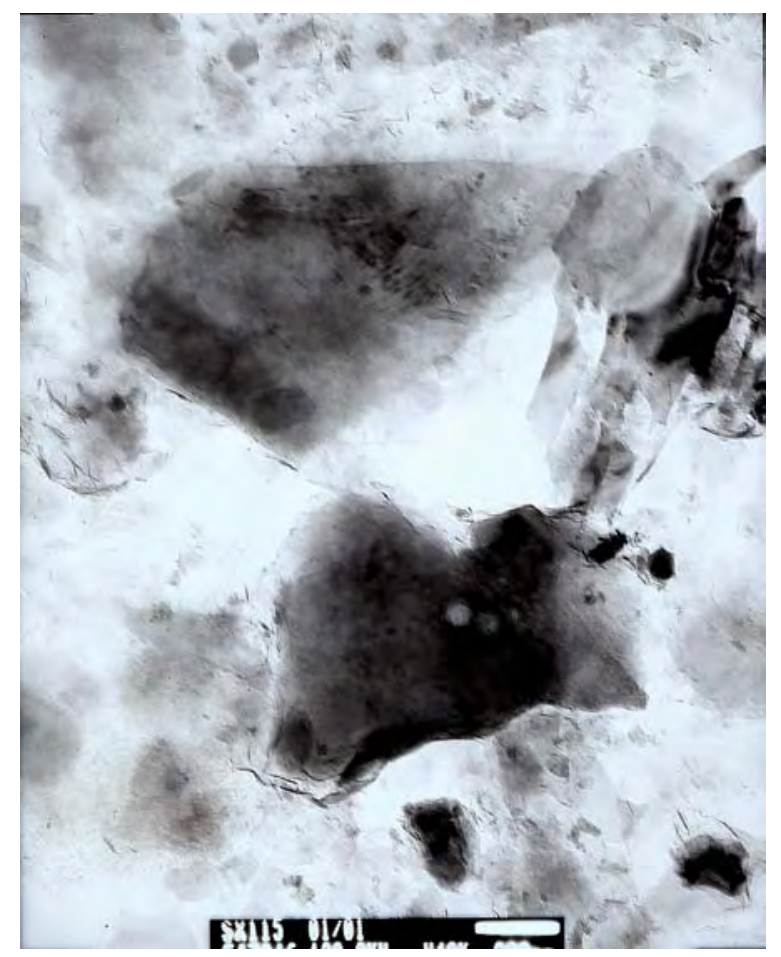

Figure 4.15. Kaolinite Particle (Bottom) with Trace Amounts Of Iron (III) $(\sim 1 \mu \mathrm{m})$ and Platy Illite Particle (Top) from Sample 22D. The kaveinite particle is about $1 \mu \mathrm{m}$ across.

\subsubsection{Water Potential or Soil Suction}

A key hydrologic measurement in the vadose zone is the soil-water matric potential. Differences in water potential indicate direction of flow and, when coupled with hydraulic conductivity measurements, can determine the magnitude of the water flux (i.e., drainage or recharge). Matric potential is formally defined as "the amount of work that must be done per unit of soil solution to transport, reversibly and isothermally, an infinitesimal quantity of water from a pool of soil solution at a given elevation above the water table at atmospheric pressure to the soil pores at the same elevation and pressure" (SSSA 1997). When the work (or energy) is expressed on a weight basis (i.e., energy per unit weight), the matric potential is expressed in units of length (i.e., meters or centimeters). Matric potential is always negative, i.e., energy is gained in going from a saturated solution to unsaturated soil pores, because of adsorptive forces and capillarity of porous material. Soil matric suction (or as expressed in this report, soil suction) is the absolute value of matric potential and is used here for convenience to express the matric forces (potentials) as positive values. By definition, at the water table, matric suction is zero. As soil dries, soil suction increases. 
Soils of varying texture (i.e., sands and silts) can have widely different water contents yet have the same soil suction value. For example, when sand or silt materials are in equilibrium with a water table that is 1 meter below the soil surface, the sand can have a water content of less than $10 \mathrm{vol} \%$, whereas the silt, at the same elevation and hydrostatic pressure could have a water content twice that of the sand (e.g., $20 \mathrm{vol} \%$ or more). Thus, a silt layer can be readily distinguished in a sandy soil profile because of its elevated water content. Soil can have virtually unchanging water content and still transmit significant amounts of water; thus, water content is not a good indicator of water flux and cannot be used directly to predict flow rates in the vadose zone. For these reasons, it is important to couple soil suction information with other hydrologic properties to assess the flow rates and directions in vadose zone soil.

Unsaturated flow properties include unsaturated hydraulic conductivity and water retention characteristics (relationship between water content and matric suction values). Analogous to saturated flow where the advective flux is the product of the saturated hydraulic conductivity and the gradient of the hydrostatic head, in unsaturated sediment the advective flow is the product of the unsaturated conductivity and the matric potential (or suction) gradient. The suction gradient defines the direction of flow (from areas of low to high suction). When layered soil is draining under the influence of gravity, the matric suction value is generally constant throughout the profile, and the drainage or recharge flux is equal to the unsaturated hydraulic conductivity. This is called a unit gradient condition. Such a condition is known to exist at Hanford Site waste sites, where soil has been kept bare and winter rains drain through the profile (Gee et al. 1992). However, neither unsaturated hydraulic conductivity or water retention characteristics have been measured directly for sediment in or around the SX Tank Farm. Only water content has been measured by neutron logging in dry wells and nearby Resource Conservation and Recovery Act (RCRA) monitoring wells. Unfortunately, water content does not provide direct hydraulic characterization of the vadose zone in the tank farm.

As a part of the Vadose Zone Characterization Project, split-spoon samples of vadose zone sediment taken from the two clean boreholes (299-W22-48 and 299-W22-50) and this borehole, 299-W23-19, were used for chemical and physical analysis. Selected cores from the three boreholes were subsampled using the filter paper method to determine soil suction. The analysis was similar to that provided for borehole 41-09-39 (Serne et al. 2002b and Myers et al. 1998). Soil suction results for the three boreholes are shown in Figure 4.16. The soil suction data are reported as head values in feet, consistent with reported depths bgs.

An equilibrium line also is shown on Figure 4.16. Measured soil suction values to the left of the equilibrium line indicate that drainage (recharge) is occurring whereas values to the right of the line indicate that evaporation (drying or upward flow) conditions persist. For the two RCRA wells, 299-W2248 and 299-W22-50, it appears that water from winter rains (or perhaps other sources) has penetrated the soil profile to depths of about 30.5 to 42.7 meters (100 to 140 feet bgs). However, below that depth the sediment from these two uncontaminated boreholes is significantly drier (i.e., to the right of the equilibrium line) compared to the sediment found in the contaminated borehole 299-W23-19.

For borehole 299-W23-19, the suctions are low, or sediment is relatively wet, throughout the profile, and drainage is predicted to be the highest of any of the three boreholes. Unit gradient conditions persist throughout the profile for sediment from borehole 299-W23-19 but not for the two clean boreholes outside the tank farm operations area. If unsaturated hydraulic conductivity values were known for the sediment in borehole 299-W23-19, recharge rates could be estimated. 
Based on values obtained from hydraulic characterization of similar sediment (Rockhold et al. 1997), the net water infiltration (recharge) rates at the three sites appear to be in the range from 5 to 500 millimeters per year. Better quantification of recharge at these boreholes requires in situ or direct measurements of unsaturated hydraulic conductivity or perhaps direct quantification of the water flux. An approach to understanding the suction profiles and their implication for deep drainage at each borehole is provided in the following paragraphs.

Drainage (recharge) at each of the borehole sites can be predicted based on location and surface conditions. Soil at undisturbed sites at the Hanford Site recharges relatively little water to the underlying water table where the surface is dominated by native shrub-steppe vegetation. In contrast, Hanford Site soil does recharge water all the way to the water table (and more water) where the surfaces have been disturbed and vegetation has been removed, as is the case at tank farms (Gee et al. 1992).

As presented in the above paragraphs, soil suction was measured in selected samples from three boreholes. The two boreholes drilled outside the SX Tank Farm were drilled in areas where the soil has been disturbed, but it has not been covered with gravel and some revegetation has occurred. Therefore, the soil suction results for vadose sediment samples from those two boreholes are expected to show less recharge than results from sediment inside the tank farm where the soil has been disturbed, denuded of vegetation, and covered with gravel. Figure 4.16 shows that soil suction results from sediment from wells 299-W22-48 and 299-W22-50, located outside the tank farm, show less recharge than results from well 299-W23-19, located within the SX Tank Farm.

Recharge conditions can be indexed by the soil suction profiles. The lower and more uniform the suction values, the more recharge is expected. The higher the suction values, the less recharge is expected. Based on known soil characteristics from analog sites at Hanford, if soil suction values exceed 10 meters (30 feet) it is unlikely that recharge is occurring at any significant rate.

At least two complications exist at sites in tank farms that might affect recharge rates. First, radioactive waste produces heat that can dry soil significantly, moving water away from the tank and the waste plume. Second, there are potential water line leaks that can add water to the soil and create fastflow pathways that move water vertically downward at rates far in excess of that predicted by winter rains and snowmelt events. These complications can either dry or wet the profile and change the soil suction values significantly.

Since the two clean boreholes, 299-W22-48 and 299-W22-50, are not close to the tank farms, neither should be affected by subsurface heat loading. However, both could be affected by water-line leakage. The wetter suction values at a depth of 51.8 meters (170 feet) for borehole 299-W22-48 may be the result of deep wetting at an adjacent site where a 500,000 gallon water-line leak occurred in 1998. The observed profiles, however, suggest that in general, neither borehole location has been directly affected by water-line leakage (with the possible exception of the 170-foot depth for 299-W22-48) because both show increased suction with depth, opposite that which would be expected if water-line leakage were occurring at either site.

For borehole 299-W23-19, the soil suction profile indicates that the sediment is wet from top to bottom. It does not appear that there is any significant effect of heat loading or drying on the suction 
profile. Tank SX-115 contained lower activity waste (nearly a factor of 10 less than waste found in tank SX-108), so the corresponding heat load is expected to be less. In contrast to borehole 299-W23-19, data collected from borehole 41-09-39 (Serne et al. 2002b), showed considerable variability in suction values, with nearly half of the suction values to the right of the equilibrium line, indicating that the sediment had either dried before sampling or was not in equilibrium with a draining profile or both.

It cannot be determined unequivocally from the available data if water-line leakage or meteoric water sources, or a combination of both, are responsible for the low soil suction values at borehole 299-W23-19 near tank SX-115. However, for this location, an estimated recharge rate of between 50 to 100 millimeters per year would be in line with the observed drainage rates from bare gravel surface soil at Hanford (Gee et al. 1992).

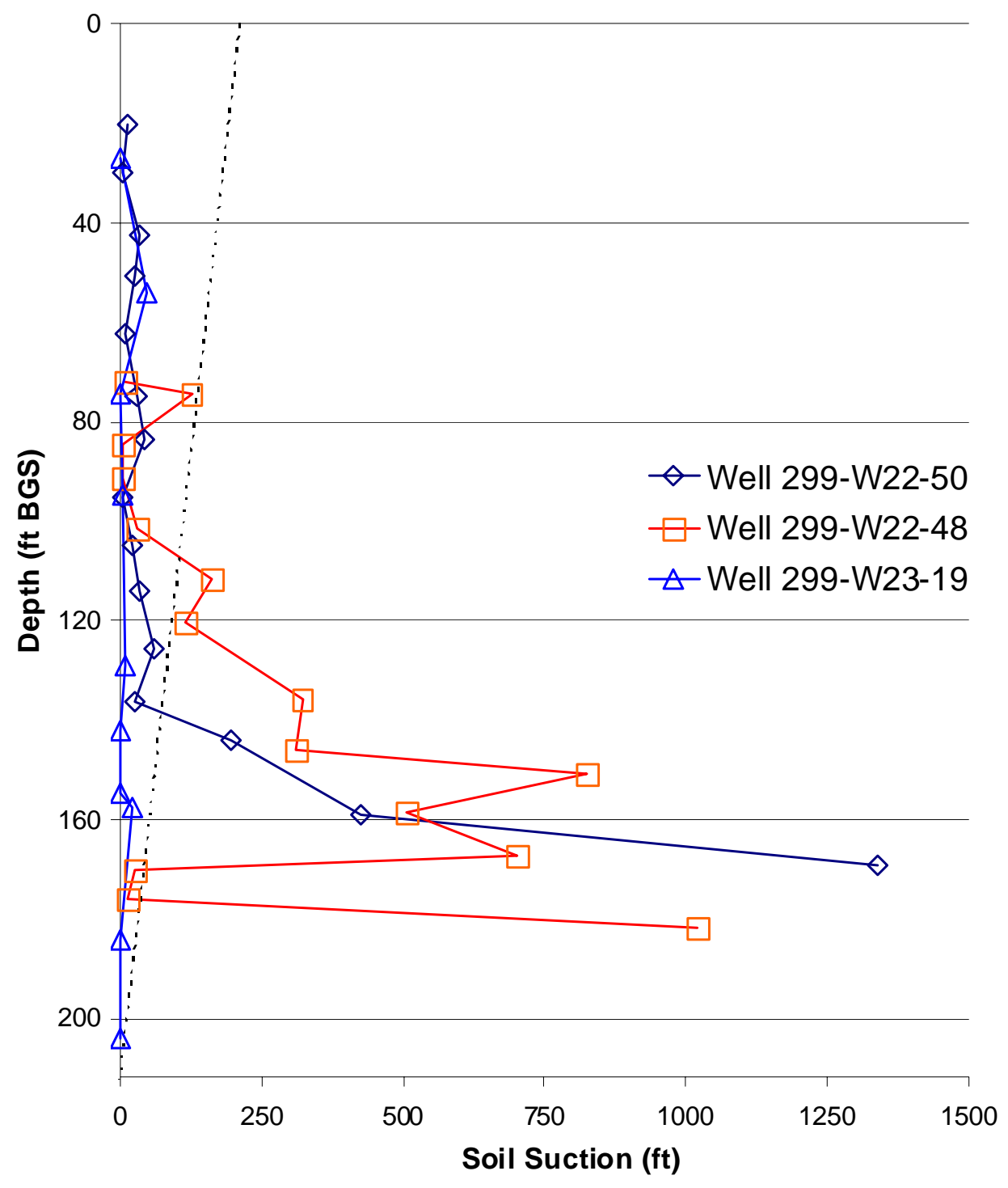

Figure 4.16. Soil Suction Profiles for Boreholes 299-W22-48, 299-W22-50, and 299-W23-19. Dashed line is the equilibrium soil suction line. Drainage occurs only for values left of the equilibrium line. 
In summary, suction values from cores taken from three boreholes near and in the SX Tank Farm provide insight into deep drainage (recharge) conditions at and near Hanford Site tank farms. Outside the SX Tank Farm, meteoric water apparently has not penetrated much past the 100-foot depth. However within the tank farm, near tank SX-115, water from natural and/or manmade sources appears to have penetrated to the water table.

\subsubsection{Results of Semi-Volatile Organic Analyses}

Although the holding times for the samples were exceeded and the samples reached ambient temperature during shipment, PNNL scientists felt that testing should proceed and that the results would be useful indicators as to whether there were regulated organic compounds in the contaminated vadose zone sediments. Analyses of the six samples and the two field/trip blanks showed that no regulated semivolatile organics were present at detectable levels. Also, there were no unidentified peaks in the chromatograms that suggested similar organic compounds. For the background sample from the backfill (B8809-8C [27.5 ft bgs]), there was an indication that bis(2-Ethlhexyl)phthalate might be present at 46 $\mu \mathrm{g} / \mathrm{g}$ levels in the backfill sample. This value is below the quantitation value of $330 \mu \mathrm{g} / \mathrm{g}$ but above the minimum detection limit of $36 \mu \mathrm{g} / \mathrm{g}$. One of the field/trip blanks showed two small unidentified peaks on its chromatogram that were estimated to represent $\sim 18 \mu \mathrm{g} / \mathrm{L}$ each of organic compounds similar to semivolatile compounds that were used in laboratory method calibration.

Based on the six semi-volatile organics analyses, no significant concentrations of mobile, regulated, semi-volatile organic compounds from tank liquors reside in the vadose zone under tank SX-115. 


\subsection{Contaminant Distribution at Borehole 299-W23-19 as Compared with Contaminant Distribution at Nearby Wells}

This section discusses the results of this study with respect to in situ distribution coefficients and the distribution of contaminants in borehole 299-W23-19 as compared with contaminant distributions in nearby boreholes.

\subsection{In Situ Distribution Coefficients}

The analytical data collected in this study were used to estimate in situ distribution coefficients for several contaminants. These estimates were made using the acid and water extract results. The estimate relies on the assumption that the acid extract gives the total quantity of contaminant in the sample; that is, the acid extract contains all the contaminant precipitated or adsorbed onto the sediment plus any contaminant in the porewater. The estimate also assumes that the 1:1 water extracts contain the amount of contaminant in the porewater and the extraction procedure does not remove any contaminant precipitated or adsorbed on the sediment.

For constituents that are moderately or strongly sorbed, the amount of contaminant in the porewater is small relative to the amount on the sediment and there is no need to subtract the small amount in the porewater from the total amount extracted by the acid extraction. In this case, the desorption $\mathrm{K}_{\mathrm{d}}$ is simply the ratio of the amount of mass in the acid extract to the amount in the dilution-corrected water extract. For constituents that show little interaction with the sediment (e.g., technetium-99), the amount of contaminant in the porewater is relatively large compared to the total that was extracted by the acid procedure. For these cases, the amount of contaminant in the porewater must be subtracted from the total amount to obtain the amount of contaminant sorbed on the sediment.

Table 5.1 shows the estimated desorption $\mathrm{K}_{\mathrm{d}} \mathrm{s}$ derived from the measurements described in this report. The technetium- 99 desorption $K_{d}$ s are consistently very low, indicating that the technetium- 99 is not interacting with the sediment. The $\mathrm{K}_{\mathrm{d}}$ for the deepest sample (39D) may indicate a slight interaction of technetium-99 with the sediment at this depth. Alternatively, the deepest sample is from a depth that was below the historical water table and the technetium-99 in that sample may be the result of residual groundwater. In general, the technetium- $99 \mathrm{~K}_{\mathrm{d}} \mathrm{s}$ in Table 5.1 suggest that technetium-99 is not interacting with the Hanford Site sediment and will travel with the water that is slowly percolating through the vadose zone. The calculated technetium- $99 \mathrm{~K}_{d} \mathrm{~s}$ are very similar to those summarized by Kaplan and Serne (1999) and Kaplan et al. (1995).

The calculated $\mathrm{K}_{\mathrm{d}}$ values for chromium, and to a lesser extent for uranium, indicate a zone between 21.3 and 32 meters (70 and 105 feet bgs) in which chromium is much more mobile than in the overlying and underlying sediment. This is in agreement with the depth distribution of chromium (Figure 4.5), which indicates that though chromium concentrations are elevated at a depth of above 21.3 meters (70 feet), the bulk of chromium migrated through the depths between the base of the tank and $\sim 32$ meters (105 feet) and precipitated or sorbed on the sediment between depths of $\sim 32$ and 39.6 meters (105 and 130 feet; see Figure 4.5). 
Table 5.1 Quantification of Desorption Potential for Major Contaminants in Borehole 299-W23-19

\begin{tabular}{|c|c|c|c|c|c|c|c|c|}
\hline \multirow{2}{*}{$\begin{array}{c}\text { Sample } \\
\text { ID }\end{array}$} & \multirow{2}{*}{$\begin{array}{c}\text { Depth } \\
(\text { ft bgs })^{2}\end{array}$} & \multirow{2}{*}{$\begin{array}{l}\text { Lith. } \\
\text { Unit }\end{array}$} & \multicolumn{6}{|c|}{ Kd Values $(\mathrm{mL} / \mathrm{g})^{1}$} \\
\hline & & & ${ }^{99} \mathrm{Tc}$ & $\mathbf{U}$ & $\mathrm{Cr}$ & As & Se & Mo \\
\hline $17 \mathrm{DE}$ & 64.75 & H1a & $<$ detect $^{3}$ & 155 & 2110 & 30.8 & $<6.13$ & 1.15 \\
\hline 17DE-Dup & 64.75 & $\mathrm{H} 1 \mathrm{a}$ & $<$ detect & 67 & 3531 & 10.4 & $<$ detect & $<0.88$ \\
\hline $18 \mathrm{E}$ & 69.45 & $\mathrm{H} 1 \mathrm{a}$ & $<$ detect & 59 & 84 & 15.8 & $<$ detect & 0.54 \\
\hline $18 \mathrm{CB}$ & 70.70 & $\mathrm{H} 1 \mathrm{a}$ & $<$ detect & 32 & 5.45 & 11.2 & $<$ detect & 0.56 \\
\hline 18CB-Dup & 70.70 & $\mathrm{H} 1 \mathrm{a}$ & $<$ detect & 35 & 8.44 & 12.6 & $<$ detect & $<0.63$ \\
\hline $19 \mathrm{H}$ & 73.25 & $\mathrm{H} 1 \mathrm{a}$ & 0.01 & 8 & 0.40 & 2.10 & 2.11 & 0.09 \\
\hline $19 \mathrm{G}$ & 73.75 & $\mathrm{H} 1 \mathrm{a}$ & 0.03 & 30 & 0.92 & 5.74 & $<1.36$ & 0.06 \\
\hline $20 \mathrm{E}$ & 79.75 & $\mathrm{H} 1$ & 0.03 & 40 & 1.47 & 1.64 & $<0.91$ & 0.09 \\
\hline $26 \mathrm{~A}$ & 104.95 & $\mathrm{H} 2$ & $<0.01$ & 17 & 1.59 & 5.34 & $<3.26$ & 0.05 \\
\hline $31 \mathrm{G}$ & 129.15 & $\mathrm{PPlz}$ & 0.02 & 125 & 1107 & 709 & $<0.27$ & 0.79 \\
\hline $31 \mathrm{~A}$ & 132.15 & $\mathrm{PPlz}$ & -0.03 & 228 & 3484 & 238 & $<0.93$ & 1.71 \\
\hline $36 \mathrm{D}$ & 147.45 & $\mathrm{PPlz}$ & 0.00 & 117 & 5774 & 269 & $<1.90$ & 0.68 \\
\hline $38 \mathrm{G}$ & 152.10 & $\mathrm{PPlz}$ & 0.04 & 154 & 4758 & 360 & $<2.77$ & 1.01 \\
\hline $38 \mathrm{~A}$ & 155.10 & $\mathrm{PPlz}$ & $<0.00$ & 67 & $>4505$ & 18.2 & $<$ detect & 0.46 \\
\hline 39D & 155.35 & $\mathrm{PPlz}$ & 0.09 & 23 & 1659 & 246 & 6.25 & 3.09 \\
\hline
\end{tabular}

\subsection{Contaminant Distribution in Borehole 200-W23-19 and other Nearby Wells}

As stated in the introduction, this report is one in a series of four reports issued in FY 2002 that describes the sampling and analysis activities associated with five boreholes drilled in or adjacent to the SX Tank Farms. The analytical results of samples from borehole 299-W23-19 are described in this report; detailed results from wells 299-W22-48 and 299-W22-50 are described in Serne et al. 2002a, results from well 299-W23-234 (also known as 41-09-39) are in Serne et al. 2002b, and results from borehole C3082 (also known as the SX-108 slant borehole) are described in Serne et al. 2002c. This section summarizes the contaminant distributions found in borehole 299-W23-19 and compares those distributions with contaminant distribution at the other nearby boreholes.

Boreholes 299-W22-48 and 299-W22-50 were drilled and completed as Resource Conservation and Recovery Act (RCRA) groundwater monitoring wells in fiscal year 2000 east of the SX Tank Farm (see Figure 2.1 for location). They were drilled in uncontaminated sediment and, as such, serve as baseline wells to compare with those drilled in contaminated areas of the tank farm. Both boreholes were continuously cored during drilling. 
Borehole 299-W23-234 (or 41-09-39) was originally drilled in December 1996 adjacent to the southeast edge of tank SX-109 to a depth of 40 meters (131 feet). The purpose of the borehole was to determine the presence of cesium-137 at depths of 20 to 40 meters (66 to 131 feet). The borehole was deepened in 1997 to a depth of 68.6 meters and split-spoon samples were collected wherever possible. The borehole was decommissioned in 1999 at which time samples were obtained from portions of the borehole that were previously unsampled.

Borehole C3082 was installed adjacent to tank SX-108 in 2000. Tank SX-108 had leaked between 2,400 and 35,000 gallons in 1962 (HNF-EP-0182, Rev. 153). Borehole C3082 was drilled at an angle and aligned to pass beneath the tank, through a zone of high contamination, and terminate at a depth of 45 meters (148 feet) in the Plio-Pleistocene unit. Split-spoon samples were obtained from 16 zones in the borehole.

Figure 5.1 illustrates the stratigraphy encountered in all five boreholes. Boreholes 299-W22-48, 299W22-50, and 41-09-39 encountered the same stratigraphic and lithologic sequences; namely, from bottom to top, (1) fluvial, variably cemented sandy gravel to conglomerate of the Ringold Formation, member of Wooded Island, unit E; (2) interbedded fluvial sands and overbank sands and muds of the Ringold Formation member of Taylor Flats; (3) basaltic pebbly sand interspersed with layers of mud and calcium carbonate representing the lower Plio-Pleistocene unit; (4) the upper Plio-Pleistocene overbank alluvium and paleosols consisting of fine to very fine-grained sand intercalated with silty fine sand and silt/clay; (5) the Hanford formation $\mathrm{H} 2$ unit consisting of lower fine sand and mud sequences; and (6) the Hanford formation $\mathrm{H} 1$ unit which is predominantly sandy gravel to gravelly sand, and (7) the Hanford formation H1a unit which consists of several glacial-fluvial upward grading, coarse sand to silty fine sand sequences. Backfill materials consisting of Hanford formation silt, sand and pebbles overlie the Hanford formation H1a unit at borehole 41-09-39.

Borehole 299-W23-19 encountered essentially the same stratigraphy and litholgies as the aforementioned boreholes except that the upper Ringold Formation is missing in borehole 299-W23-19. Borehole C3082 penetrated backfill and the Hanford formation and terminated in the upper PlioPleistocene unit.

Analytical results for moisture content, $\mathrm{pH}$, electrical conductivity, nitrate, sodium, chromium, and technetium concentrations in 1:1 sediment-to-water extract were the main indicators of tank leak contamination.

Although there was some correlation between moisture content and the presence of contamination, the relationship was not straightforward because the moisture content also reflects lithology changes. Fine-grained sediment tended to have higher moisture contents than coarse-grained sediment and those differences were mixed with moisture differences due to leaked fluids. In addition, for samples near tanks, heat from the tanks could have changed the moisture content. Therefore, moisture by itself was not considered a good indicator of contamination although moisture content tended to be higher in contaminated zones in boreholes 299-W23-19, 41-09-39, and C3082.

The $\mathrm{pH}$ from 1:1 water extracts versus depth for all five boreholes is shown in Figure 5.2. The figure shows that natural $\mathrm{pH}$ values of uncontaminated sediment are between about 7.0 and about 8.5 with a few 

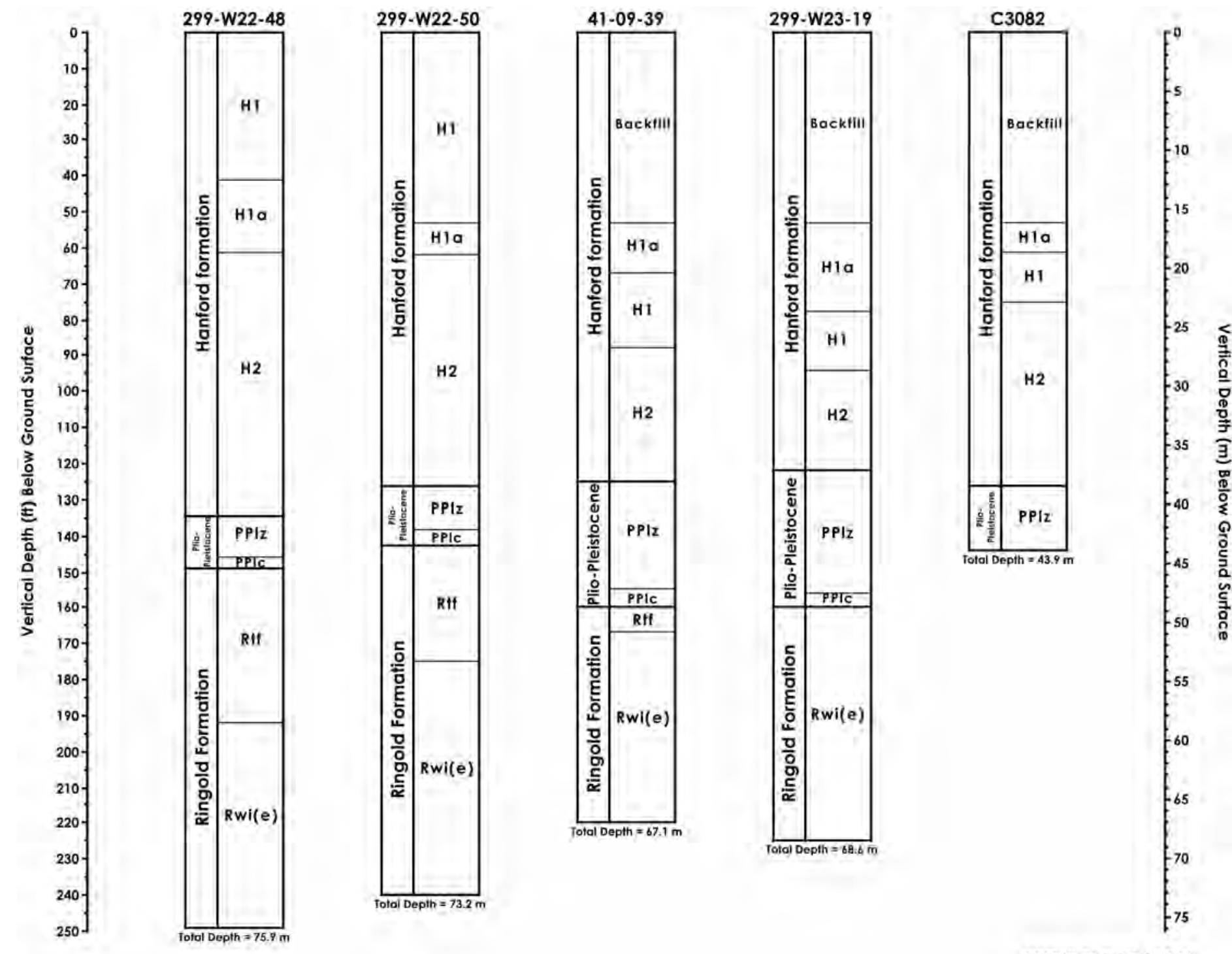

2002/DCL/W23-19/021

Figure 5.1 Stratigigraphic sequences encountered in five new boreholes at the S-SX tank farms. 



Figure $5.2 \mathrm{pH}$ versus depth in five new boreholes at the SX tank farm. 
samples extending to near 9.0. The $\mathrm{pH}$ of Hanford formation sediment in equilibrium with atmospheric carbon dioxide and calcite (calcium carbonate, which is common in Hanford Site sediment) would be 8.3.

The $\mathrm{pH}$ values of samples taken from depths above the bottom of tanks SX-109 and SX-115 (less than about 18.2 meters [60 feet]) are within the range of natural $\mathrm{pH}$ values. At both borehole 41-09-39 and $\mathrm{C} 3082$, the highest $\mathrm{pH}$ values are between 9.2 and 9.8 and occur just below tanks SX-109 and SX108 to 24.4 to 25.9 meters ( 80 to 85 feet) depths. In borehole 299-W23-19, elevated pH, from 9.1 to 9.7 , occurs between 22.8 and 26.2 meters (75 and 86 feet) depths, similar to tanks SX-108 and SX-109, and between 30.2 and 32 meters ( 99 and 105 feet) depths. Below a depth of about 24.4 to 25.9 meters (80 to 85 feet) at tanks SX-108 and SX-109 and below about 32 meters (105 feet) beneath tank SX-115, pH values are within the range of natural, uncontaminated Hanford Site sediment. Thus, elevated $\mathrm{pH}$ extends somewhat deeper in borehole 299-W23-19 than in boreholes 49-09-39 and C3082.

In none of the contaminated boreholes is the $\mathrm{pH}$ as high as might be expected for tank liquors completely saturating sediment $(\mathrm{pH}>13$, Serne et al. 1998). This may result from neutralizing reactions with the sediment and/or carbon dioxide in the vadose zone. Because of these possible neutralizing reactions, $\mathrm{pH}$ is not considered a good indicator of the extent of contamination.

The electrical conductivity results for the five boreholes in the SX tank farm are shown in Figure 5.3. The natural, dilution-corrected electrical conductivity in boreholes 200-W22-48 and 299-W22-50 is between about 200 and $6000 \mu \mathrm{S} / \mathrm{cm}$. (Because the 1:1 water extract values are dilute with respect to the natural porewater, the diluted value is multiplied by the dilution factor [the amount of water added] to obtain the natural value for the sediment [Serne et al. 2002a].) One sample at a depth of about 18.3 meters (60 feet) in borehole $299-\mathrm{W} 22-50$ shows $\mathrm{pH}$ and electrical conductivity values greater than the other samples from the two uncontaminated boreholes. The electrical conductivity of that sample may or may not be natural due to dissolution of evaporite minerals in the sample (Serne et al. 2002a). It could also represent lateral spreading of waste from past liquid discharges to cribs.

The electrical conductivity versus depth profiles for boreholes 41-09-39 and C3082 are similar with respect to the stratigraphy. The profile for 41-09-39 shows that tank fluid dominates porewater to a depth of 38.7 meters (127 feet) and electrical conductivity is elevated to depths of about 40.2 meters (132 feet) below which are natural values. Similarly, electrical conductivity shows that tank fluid dominates porewater to a depth of 39.1 meters (129 feet) in borehole C3082, though the two deeper samples still have slightly elevated electrical conductivity. Note that electrical conductivity of the sediment beneath tank SX-108 (C3082) is an order of magnitude greater than that beneath tank SX-109 (41-09-39). The data from these two boreholes suggest that the leading edge of the contamination has reached a depth of 40.2 meters (132 feet) in borehole 41-09-39 and is below the total depth of 43.9 meters (144 feet) in borehole C3082. For both boreholes, however, the bulk of the contamination is higher, between depths of 24.4 and 39.6 meters (80 and 130 feet) (41-09-39) and 22.8 and 36.6 meters (75 and 120 feet) (C3082).

The profile from borehole 299-W23-19 is somewhat different than that of the other two boreholes. The electrical conductivity in borehole $299-W 23-19$ is elevated from about 65 - to 156-foot depths. The profile shows that the contaminant plume has reached the bottom of the upper Plio-Pleistocene unit at a depth of 47.5 meters (156 feet) and the bulk of contamination is within the upper Plio-Pleistocene unit between depths of about 36.6 and 47.5 meters (120 and 156 feet). This is deeper than the bulk of contamination as shown by electrical conductivity from boreholes 41-09-39 and C3082. 

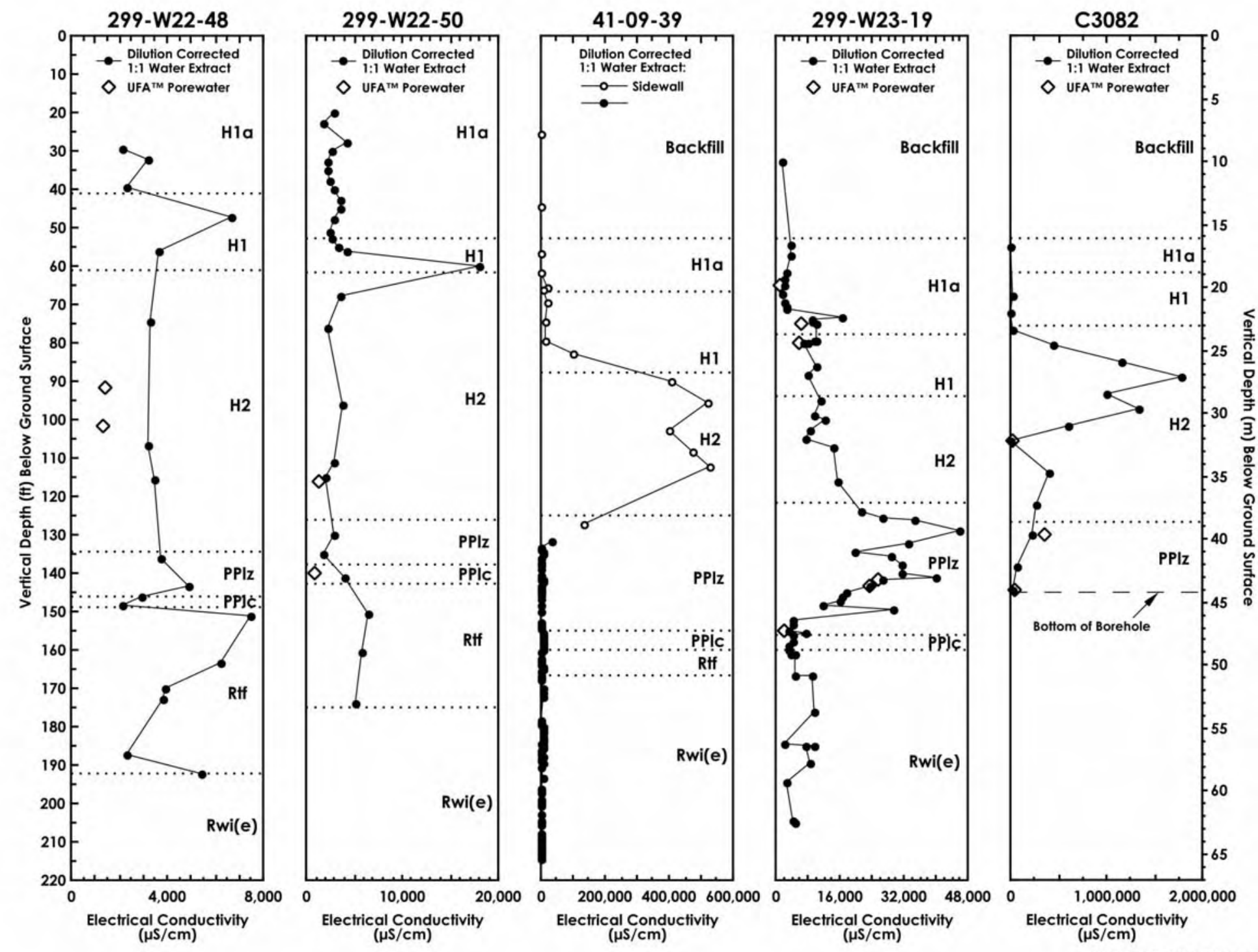

Figure 5.3 Dilution corrected electrical conductivity versus depth in five new boreholes at the SX tank farm. 
Nitrate is the most concentrated anion in tank liquors. Its high concentrations and its high mobility in the vadose zone make nitrate one of the most sensitive markers of migrating tank waste through the vadose zone. Figure 5.4 shows the concentration of nitrate versus depth in the five new boreholes at the SX Tank Farm. Also, sodium is the dominant cation in tank fluids and is somewhat mobile in the vadose zone environment. Figure 5.5 shows the concentration of sodium versus depth in the SX Tank Farm boreholes. Both nitrate and sodium closely resemble the electrical conductivity profiles.

In borehole 41-09-39, elevated nitrate is first encountered at a depth of 19.8 meters ( 65 feet) and is highest between 24.4 and 38.7 meters ( 80 and 127 feet). Likewise, sodium is elevated from depths of about 24.4 and 38.7 meters ( 80 to127 feet), though moderate sodium values exist to a depth of about 46.9 meters (154 feet), below which natural sodium concentrations are present. These deeper sodium concentrations, between 39.6 and 47.5 meters (130 and 156 feet), may be due to drag down during drilling of the borehole (Serne et al. 2002b). Nitrate in samples from C3082 is elevated through total depth of the borehole ( 44.2 meters [145 feet]) and sodium has severely impacted the vadose zone to a depth of about 47.5 meters (130 feet). The sodium concentration is near normal in the deepest sample collected at about 44.5 meters (146 feet).

Nitrate in borehole 299-W23-19 is elevated from 19.8 meters (65 feet) to about 47.5 meters (156 feet), near the bottom of the upper Plio-Pleistocene unit. Nitrate is less than $1000 \mathrm{mg} / \mathrm{L}$ and generally less than $500 \mathrm{mg} / \mathrm{L}$ (within natural range) in samples deeper than 47.5 meters (156 feet) except for the deepest two samples. The deepest two samples are from depths below the highest recorded water table during tank farm operations. Thus, the deepest nitrate concentrations may have resulted from nitrate in a higher than current water table. In general, the distribution of nitrate with depth in borehole 299-W23-19 suggests that the leading edge of the nitrate plume is at a 156-foot depth.

Figure 5.5 shows elevated sodium concentrations beginning abruptly at 22.2 meters ( 73 feet bgs) in borehole 299-W23-19 and extending to about 30.4 meters (126 feet bgs) near the contact between the Hanford formation $\mathrm{H} 2$ unit and upper Plio-Pleistocene unit. The bottom of the sodium profile is not sharp but gradually decreases throughout the upper Plio-Pleistocene unit until natural concentrations are attained at about 47.5 meters (130 feet bgs).

In boreholes 41-09-39 and C3082, sodium is slightly elevated throughout the upper part of the boreholes but does not greatly increase until 23.2 to 25.3 meters (76 to 83 feet bgs). In borehole 41-0939 , sodium concentration decreases to natural levels at 40.5 meters (133 feet bgs); sodium remains above natural levels in borehole $\mathrm{C} 3082$ to total depth. Whereas the bulk of the nitrate contamination is in the Hanford formation $\mathrm{H} 2$ unit in boreholes 41-09-39 and C3082, the bulk of nitrate contamination is in the upper Plio-Pleistocene unit in borehole 299-W23-9. The bulk of the sodium contamination is in the Hanford formation $\mathrm{H} 2$ unit in all three boreholes.

The alkali and alkaline-earth cation profiles for the boreholes impacted by tank waste show evidence of ion exchange in the vadose zone (Figure 5.6). This is especially evident for sodium and the alkalineearth ions. The profiles show that the high sodium concentration in the leaked fluids has replaced the other major cations on exchange sites in the impacted sediment. The displaced cations are effectively pushed ahead of the sodium-bearing fluids as they migrate downward. Each profile of contaminated boreholes in Figure 5.6 show a zone of elevated sodium above a zone of relatively high alkaline-earth concentrations. 

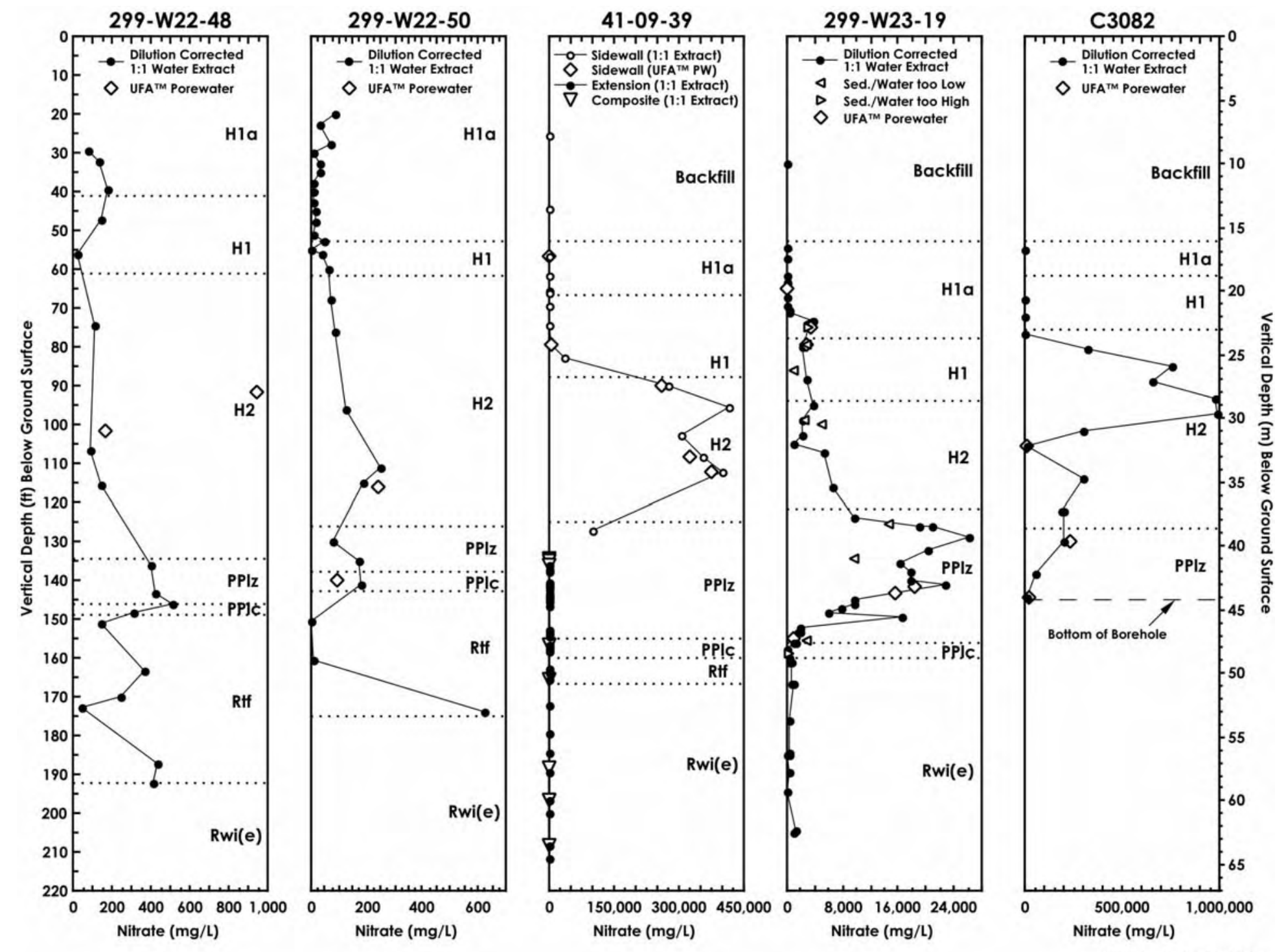

Figure 5.4 Dilution corrected nitrate concentrations versus depth in five new boreholes at the SX

2002/DCL/W23-19/024 tank farm. 

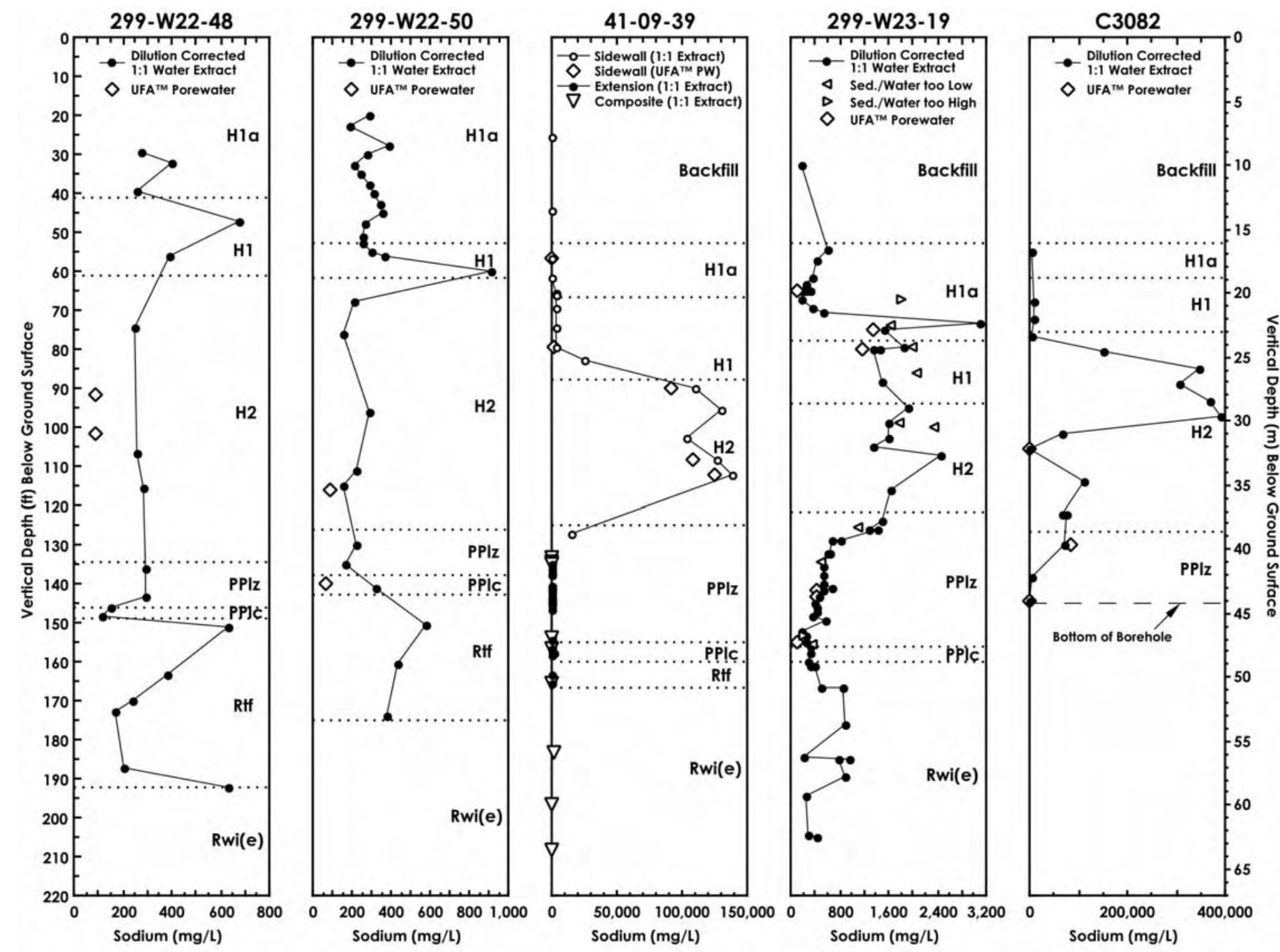

Figure 5.5 Dilution corrected sodium concentrations versus depth in five new boreholes at the SX

2002/DCL/W23-19/025 tank farm. 

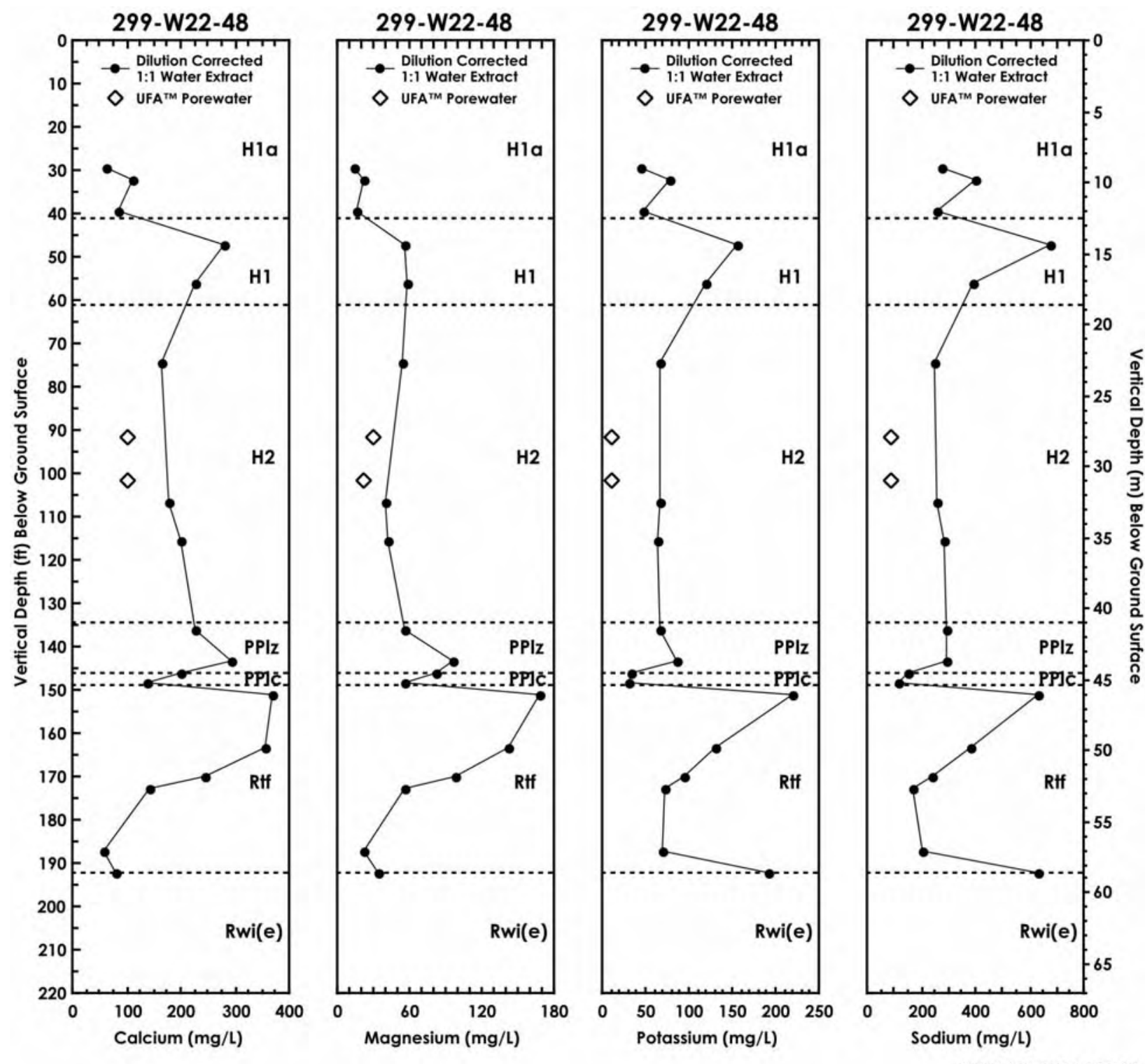

Figure 5.6a Alkali and alkaline-earth metal concentrations versus depth in four new boreholes at the SX tank farm. 

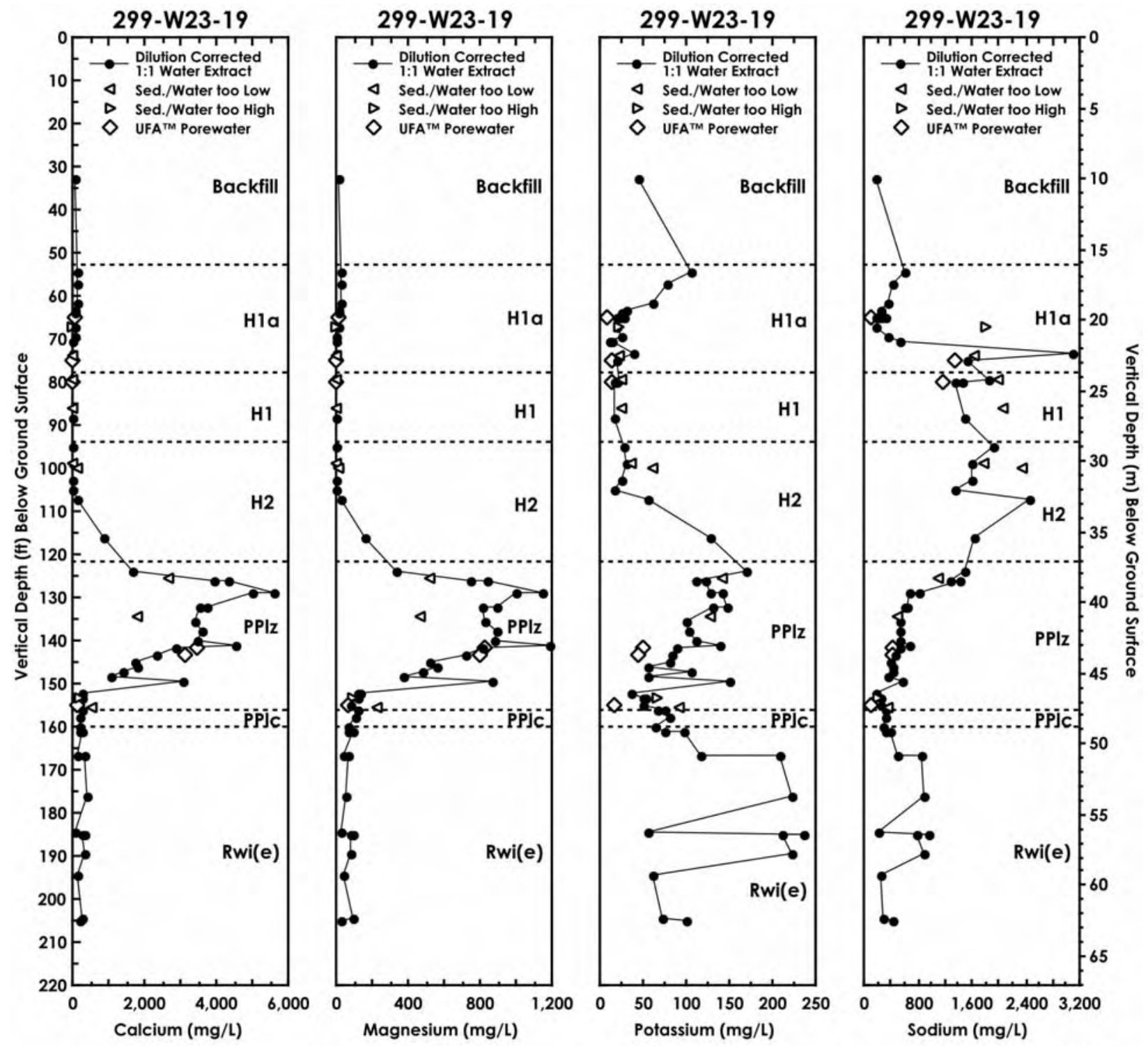

2002/DCL/W23-19/027

Figure 5.6b 

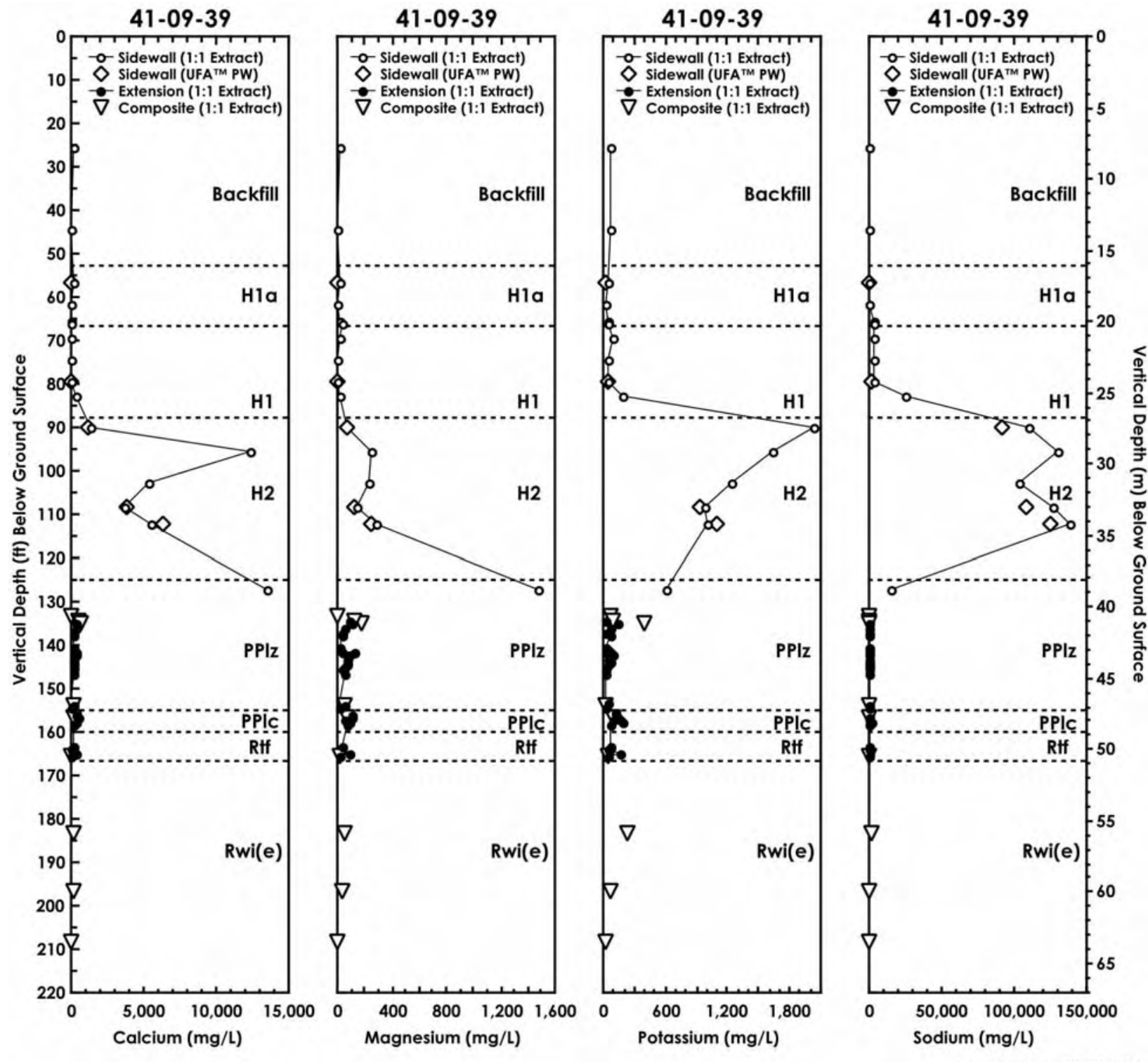

Figure 5.6c 

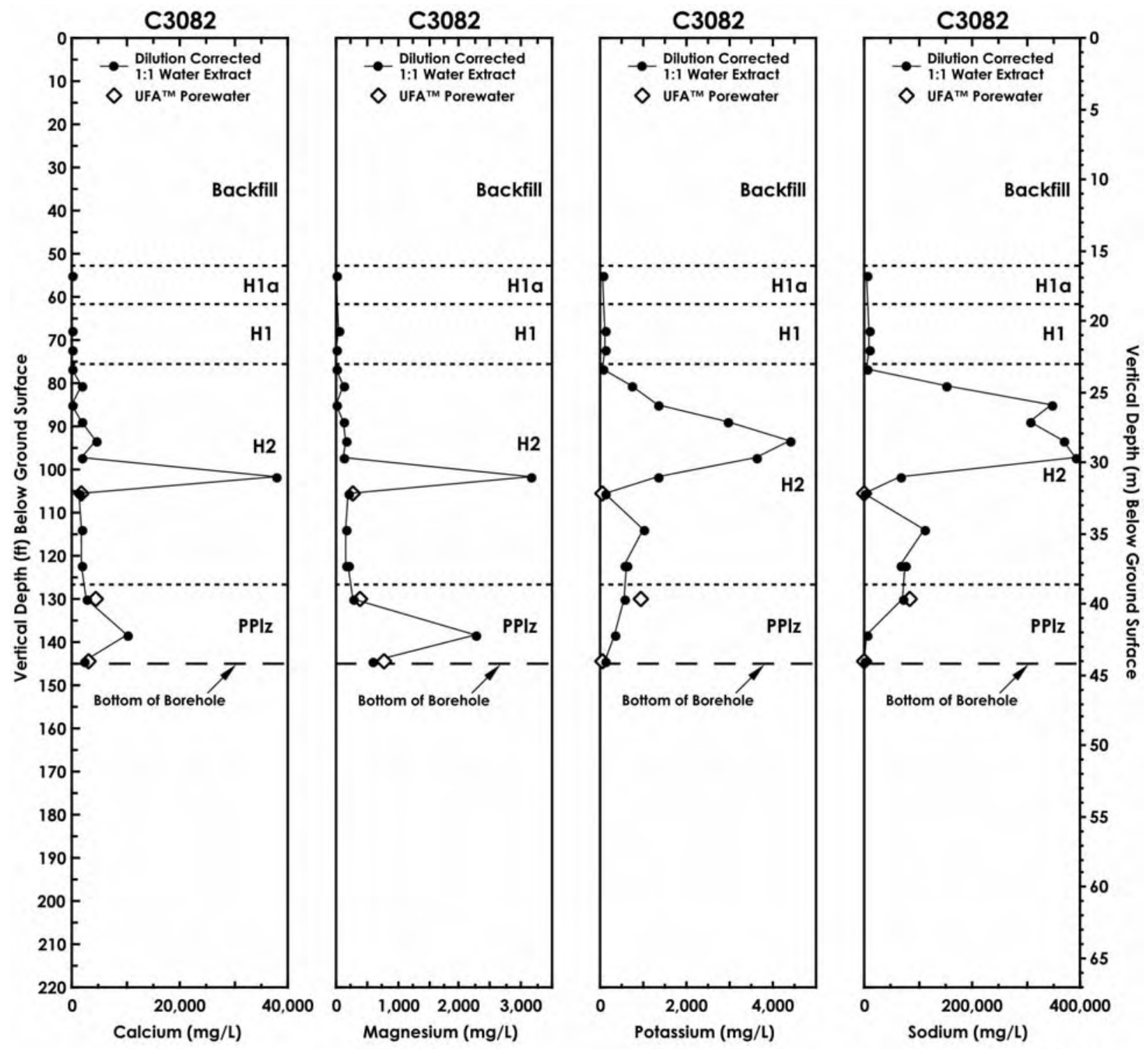

2002/DCL/W23-19/029

Figure 5.6d 

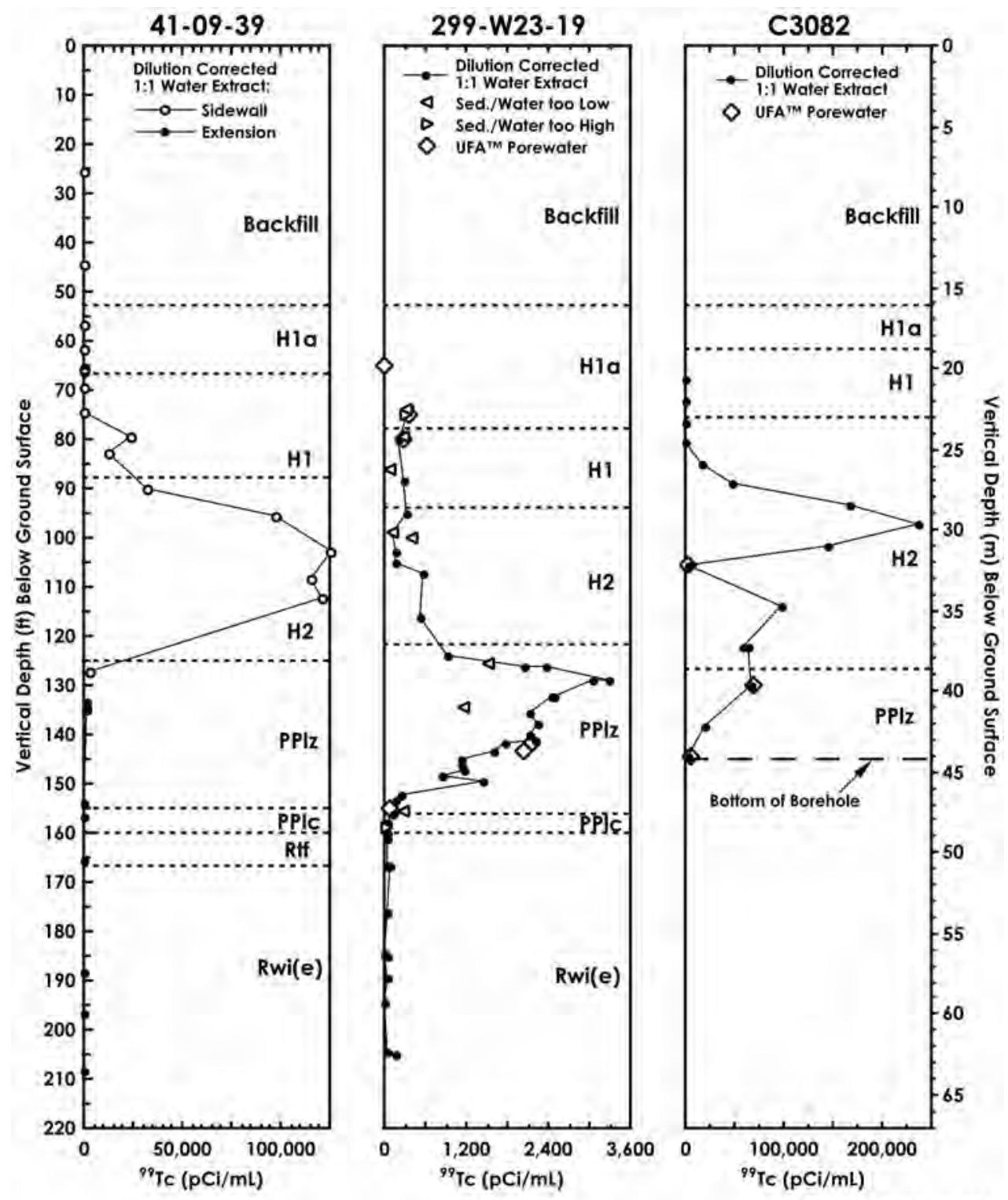

2002/DCL/W23-19/030

Figure 5.7 Dilution corrected technetium -99 concentrations versus depth in three new boreholes at the SX tank farm.

The concentration of chromium and technetium-99, two contaminants from leaked tank fluids, were found to be elevated in the vadose zone sediment. Technetium-99 is thought to be extremely mobile in the vadose zone. Figure 5.6 shows the distribution of technetium-99 versus depth for the three contaminated boreholes at SX Tank Farm. The distribution of elevated technetium-99 generally mimics that of electrical conductivity and nitrate. Using the distribution of technetium-99, the leading edge of the contaminant plume is at depths of 41.1 and 42 to 44.2 meters (135 and 138 to 145 feet) in boreholes 41-09-39 and C3082, respectively. In borehole 299-W23-19, the bulk of technetium-99 contamination is above 47.5 meters ( 156 feet bgs) in the upper Plio-Pleistocene unit, but elevated technetium-99 (between 6 and $68 \mathrm{pCi} / \mathrm{L}$ ) extends to the deepest sample. The deepest 
sample contains $176 \mathrm{pCi} / \mathrm{L}$ technetium-99, which may be a result of past interactions with technetium-99-bearing groundwaters.

In summary, $\mathrm{pH}$, chromium, and moisture distributions do not identify the leading edge of contaminant plumes in the SX Tank Farm. However, the more mobile indicators, e.g., electrical conductivity, nitrate, sodium, and technetium suggest that the leading edge of the contaminant plume is at a depth of 38.7 to 42 meters (127 to 135 feet) in borehole 41-09-39 and 42 meters (138 feet) to greater than 44.2 meters (145 feet) (deeper than the bottom of the borehole) in borehole C3082. In these two boreholes, the bulk of contamination appears to be significantly shallower than the leading edge of the plume and contamination does not seem to have migrated deeper than the base of the upper PlioPleistocene unit. In borehole 299-W23-19, the bulk of contamination is between a depth of 37.2 and 47.5 meters (122 and 156 feet), but small amounts of contamination may extend to groundwater. 


\subsection{Summary and Conclusions}

This section presents summary discussions about the characterization data from borehole 299-W2319 and conclusions drawn from the results of the study. It is hoped that the conclusions will be useful in making decisions concerning interim actions and future studies needed to make current and future tank farm operations less likely to unfavorably affect the environment.

\subsection{Physical Model of the Hydrogeology at Borehole 299-W23-19}

Borehole 299-W23-19 intersects four, generally continuous, near-horizontal principal stratigraphic units (see Figure 2.2): (1) backfill around the tanks, (2) the Hanford formation, (3) the Plio-Pleistocene unit, and (4) the Ringold Formation. Several of the layers dip toward the southwest. The Ringold Formation in borehole 299-W23-19 consists of the gravel unit E of the member of Wooded Island. Two Plio-Pleistocene units are present and are the silt-rich upper Plio-Pleistocene unit and the caleareous lower Plio-Pleistocene unit. The Hanford formation consists of three units: the lowermost Hanford formation H2 unit, the middle Hanford formation H1 unit, and the uppermost Hanford formation H1a unit. The Hanford formation H1a unit is sporadically distributed at the SX Tank Farm because it was removed during excavation and replaced with backfill.

Backfill material from construction activities associated with the SX tank farm at 299-W23-19 extends from the surface to a depth of 18.7 meters (61.5 feet). The backfill is relatively non-cohesive, friable, massive sand with minor variable mud and pebble content. The backfill has a compacted zone at its base.

The Hanford formation H1a unit (upper fine-grained sand and mud sequence) is between depths of 18.7 meters (61.5 feet) and 23.8 meters ( 78 feet). This sequence consists of several upward grading, coarse sand to silty-fine sand "rhythmites" deposited during a single cataclysmic-flood episode, or from pulses of a single flood. The content of the H1a unit in borehole 299-W23-19 is quite variable ranging from about $2.5 \%$ to greater than $23 \mathrm{wt} \%$.

The Hanford formation H1 unit (middle coarse sand and gravel sequence) occurs between depths of 23.8 meters (78 feet) and 28.7 meters (94 feet). This unit is predominantly pebbly, medium- to coarsegrained gravel. At least two layers of fine sand to muddy sand are present within this sequence. Mud content in the coarser-grained facies of the Hanford formation $\mathrm{H} 1 \mathrm{unit}$ is generally low (5 to $7 \mathrm{wt} \%$ ). The calcium carbonate content within this unit is also relatively low ( $2 \mathrm{wt} \%)$.

The Hanford formation H2 unit (lower fine sand and mud sequence) is between depths of 28.7 meters (94 feet) and 37.2 meters (122 feet). This unit is predominantly stratified, fine-grained sand to silty finegrained sand. Several interbedded, coarser-grained sands occur toward the top of the sequence. Graded rhythmite development is not as obvious in the Hanford formation H2 unit as it is in the H1a unit.

The upper Plio-Pleistocene (PPlz) unit extends from depths of 37.2 meters (122 feet) to 47.5 meters (156 feet). Most of the sediment in the upper Plio-Pleistocene unit is interpreted to be fine-grained overbank alluvium interspersed with weakly-developed paleosols. Dark carbonaceous aggregates of 
decomposed organic material and secondary iron-oxide and manganese-oxide staining are common within this unit.

The lower Plio-Pleistocene (PPlc) unit extends from depths of 47.5 meters (156 feet) to 48.8 meters (160 feet). The lower Plio-Pleistocene unit consists of weathered, dark-olive-gray, loose, basaltic, pebbly sand, interspersed with more-indurated layers of mud and calcium carbonate. The sediment from borehole 299-W23-19 is interpreted to represent the boundary between a sidestream alluvial gravel facies and the massive-carbonate facies of the lower Plio-Pleistocene unit to the north and east. The lower PlioPleistocene unit at this location is highly basaltic and coarse-grained, in contrast to the lower PlioPleistocene unit in borehole 41-09-39, 299-W22-48, and 299-W22-50 at the SX Tank Farm. The PlioPleistocene unit in the latter boreholes is less basaltic and finer-grained. Also unlike boreholes 41-09-39, 299-W22-48 and 299-W22-50, in which the calcium carbonate is dominantly a well-developed caliche layer, the calcium carbonate in borehole 299-W23-19 occurs as stringers within the coarse-grained, basaltic alluvium.

The Ringold Formation, member of Wooded Island, Unit E occurs below the Plio-Pleistocene unit from a depth of 48.8 meters (160 feet) to the bottom of the borehole. The recovered materials consist predominately of fluvial, weakly cemented, sandy gravel to well-cemented, matrix-supported conglomerate. This formation is further characterized by a yellow-brown coloration, and no reaction with hydrochloric acid. The gravel/conglomerate facies is both clast- and matrix-supported. The matrix varies from (1) sandy mud to muddy, fine sand to (2) well-sorted, quartz-rich fine to medium sand to (3) very coarse sand. In places, the basalt and granite clasts are highly weathered, and iron-oxide staining is common.

The upper Ringold Formation member of Taylor Flats is not present in borehole 299-W23-19.

The historical, peak groundwater elevation beneath the SX Tank Farm is estimated to have been about 146.2 meters ( 479.7 feet) above mean sea level based on historical water-table elevations. The highest water level occurred in 1976 at which time the water table was approximately 55 to 56 meters (182 feet) bgs or 40 meters (132 feet) beneath the bottom of the SX tanks. A secondary maximum occurred in 1984, just before the 216-U-10 Pond was decommissioned. At that time, the water table is estimated to have been about the same elevation as in 1976. In August 1999, the water table was encountered at a depth of approximately 64.5 meters (212 feet) in borehole 299-W23-19. Thus, the water table has dropped an estimated 8 to 9 meters (28.5 feet) since 1984. An examination of the hydrographs since about 1988 suggests that the water level is dropping at a rate of 0.5 to 0.6 meters per year (1.5 to 1.9 feet per year). Contamination encountered in the deepest samples from borehole 299-W23-19 may be the residual left from a shallower water table.

\subsection{Vertical Extent of Contamination}

Moisture content, $\mathrm{pH}$, electrical conductivity, and chromium, nitrate, sodium, and technetium concentrations in water extracts were used as the main indicators of the leading edge of the tank leak plume in borehole 299-W23-19. Acid extraction and x-ray fluorescence measurements were used to delineate the total inventory of contaminant distribution. For technetium-99, the water extractable and acid/total measurements were similar signifying that technetium-99 does, in fact, remain in the vadose zone porewater and interacts very little with the sediment. 
Moisture content is a direct measure of the mass of fluid in the vadose zone sediment. It is logical to assume that samples containing greater than natural moisture represent samples with an added component of leaked fluid from some source. However, as found in borehole 41-09-39, moisture content in borehole 299-W23-19 did not give a clear indication of the extent of the tank leak plume. This is because the moisture content is, in part, a function of sediment grain size as well as added contaminant fluids. Also, the moisture content of the sediments can be changed by heat generated from nuclear decay in samples near tanks and heat conducted from the tanks themselves. Generally, the natural moisture content of Hanford Site vadose zone sediment is less than $10 \mathrm{wt} \%$ with the finer-grained sediment having higher moisture contents than coarse-grained sediment. In borehole 299-W23-19, moisture content varied from about $4 \mathrm{wt} \%$ to somewhat greater than $20 \mathrm{wt} \%$ indicating a contribution of anthropogenic fluids to the sediment moisture content. However, because the additional moisture is superimposed on a variable, natural moisture distribution due to changes in lithology, and because the specific source of the additional moisture is not known, the overall moisture distribution is not a good indicator of contamination in borehole 299-W23-19.

The $\mathrm{pH}$ of water extracts and of actual porewaters obtained by ultracentrifugation was measured to delineate subsurface contaminant plumes. We anticipated that the highly caustic tank liquor would alter the sediment $\mathrm{pH}$ dramatically. The 1:1 water extract $\mathrm{pH}$ for sediment between 22.8 and 26.2 meters (75 and 86 feet bgs) (in the Hanford formation H1 unit) and 30.2 to 32 meters (99 to 105 feet bgs) (in the H2 unit) is elevated suggesting the presence of caustic waste interaction. The elevated $\mathrm{pH}$ values range from 9.2 to 9.7 in the shallower zone and from 9.1 to 9.3 in the deeper zone. The $\mathrm{pH}$ values are not nearly as high as would be expected for tank liquor completely saturating sediment. As described in Serne et al. (1998), the $\mathrm{pH}$ can reach values of $>13$ when simulated tank liquor reacts with Hanford Site sediment. One plausible explanation for the lower than expected $\mathrm{pH}$ values is that the $\mathrm{pH}$ re-neutralizes slowly with time from the slow dissolution of alumino-silicate minerals. Likewise, it also is plausible for the tank liquor to react with carbon dioxide in the air-filled porosity in the vadose zone such that an initial high $\mathrm{pH}$ is lowered over time. The $\mathrm{pH}$ profile for borehole 299-W23-19 is fairly variable and is not a smooth ascending and then descending plume. Because of this, and because there are many chemical reactions that affect $\mathrm{pH}, \mathrm{pH}$ is not considered a reliable indicator of subsurface contaminant distribution.

The electrical conductivity and nitrate concentration were measured from water extracts in an attempt to delineate subsurface contaminant distribution. The results show an obvious zone of elevated electrical conductivity and nitrate contamination starting at about 19.8 meters (65 feet bgs) and extending down to near the bottom of the upper Plio-Pleistocene unit at 47.5 meters (156 feet bgs). Below the upper PlioPleistocene unit, the electrical conductivity, nitrate and nitrite concentrations are not elevated as shown by comparison with the profiles for the two uncontaminated RCRA boreholes. Thus, the anion data, which are generally good indicators of all mobile contaminants, show that tank fluids have contacted the sediment at borehole 299-W23-19 from a depth of 19.8 to 47.5 meters (65 to 156 feet bgs). The main portion of the plume resides in the fine-grained upper Plio-Pliestocene unit. There is no evidence from the electrical conductivity or anion data that contamination has penetrated into and through the lower Plio-Pleistocene caliche unit and entered the underlying Ringold Formation sediment at this borehole. The relatively high sulfate concentrations in the lower part of the Hanford formation and upper part of the upper Plio-Pleistocene unit and the relatively high chloride concentrations in the upper Plio-Pleistocene unit, may be the result of anion exchange reactions. Nitrate from leaked tank fluids may have displaced natural chloride and sulfate from exchange sites at shallow depths and thereby pushed the natural anions to deeper zones. 
The sodium concentration was also measured and used as an indicator of subsurface contamination. Sodium is the dominant cation in leaking tank liquor and like nitrate is present at molar concentrations. The sodium profile shows elevated concentrations starting abruptly at 22.2 meters (73 feet bgs) and continuing to a depth of 38.4 meters (126 feet bgs), close to the contact between the Hanford formation H2 and upper Plio-Pleistocene units. The bottom of the elevated sodium profile is not sharp and higher than natural leachable sodium can be followed to a depth of 43.6 meters (143 feet bgs) within the upper Plio-Pleistocene unit.

The vertical distributions of the other cations (i.e., barium, calcium, potassium, magnesium, and strontium) show remarkable consistency. Each of these cations shows a large increase of waterextractable concentration at 35.5 meters (116.4 feet bgs). Unfortunately, the next shallower sample tested was from 32.6 meters (107 feet bgs) so that somewhere between depths of 32.6 and 35.5 meters (107 and 116.4 feet) the alkali and alkaline earth cations have a dramatic increase in concentration. The depth versus concentration profiles for the alkali and alkaline earth cations appear to reflect a traditional ionexchange process whereby the high sodium content of the leaked fluid has displaced the other cations, that were originally on the surface exchange sites of the natural sediment. The displaced cations were effectively pushed ahead of the sodium and almost reach the contact between the upper and lower PlioPleistocene units at 47.5 meters (156 feet bgs). Where sodium has replaced the other cations there should be low values for the water-extractable cation concentration but, unlike the sediment from borehole 4109-39, there are no obvious zones of low alkaline-earth concentrations. This is because the sodium concentration in the fluids leaked from tank SX-115 was lower than the sodium concentration in the fluids from tank SX-109. The sodium in the porewaters at borehole 299-W23-19 was not concentrated enough to remove all the alkaline earth cations, but enough alkaline-earth cations were displaced to create the ion exchange front at the leading edge of the tank fluid plume. Because the anions from the tank leak fluid are less reactive than cations, we were able to track the anions all the way to contact between the upper and lower Plio-Pleistocene units. .

The concentrations of mobile trace metals (arsenic, chromium, molybdenum, selenium, and technetium-99) were measured to map the subsurface contaminant plume. Elevated concentrations of chromium and technetium were found in the vadose zone at borehole 299-W23-19. Elevated chromium starts at 20.6 meters (67.5 feet bgs) and continues to about 4 feet below the Hanford formation - upper Plio-Pleistocene contact at 38.4 meters (126 feet bgs). The largest chromium concentration is found at 32.6 meters (107 feet bgs).

Technetium-99 is found in the porewater/water extracts beginning at 22.2 meters (73 feet bgs) and is significantly elevated down to the contact between the upper and lower Plio-Pleistocene units at 47.5 meters (156 feet bgs). Much lower technetium-99 concentrations between 6 and $68 \mathrm{pCi} / \mathrm{L}$ are found below 47.5 meters (156 feet) to the bottom of the borehole. Unlike the other indicators of subsurface contaminant distribution, technetium-99 concentration indicates that technetium-99 has migrated through the vadose zone to groundwater. The relatively high technetium-99 concentration in the deepest sample from borehole 299-W23-19 is probably the result of higher water levels in the past. The bulk of the technetium-99 contamination is located in the upper Plio-Pleistocene unit.

The arsenic and molybdenum concentrations in the porewaters show a small amount of contamination from 22.2 to 32.9 meters (73 to 108 feet bgs) (in the fine laminated sands of the Hanford formation $\mathrm{H} 2$ 
unit) when compared to background values from the uncontaminated boreholes. The fact that elevated concentrations of arsenic and molybdenum are not as deep as the technetium-99 or chromium plumes may be a result of our relatively high detection limits for arsenic and molybdenum rather than a reflection of sediment/fluid interactions.

If dilution was the sole reason for the decreases in concentrations of all the mobile constituents in the porewaters from borehole 299-W23-19, then the ratio of any two mobile constituents should remain constant with depth. Ratios of the various mobile species were calculated to test this. The ratio of technetium-99 to nitrate, chloride to nitrate, and chloride to technetium-99 are fairly constant suggesting that these three constituents migrate together through the vadose zone with little to no reaction with the sediment (alternatively, the constant ratios could also mean that the three constituents have the same $\mathrm{K}_{\mathrm{d}}$ or sorption tendencies). Ratios of relatively immobile constituents to mobile constituents tend to decrease with depth indicating various amounts of retardation. In general, the bulk of the reactive constituents chromium, molybdenum, selenium, arsenic, and sodium have reacted with the vadose zone sediment within the laminated sands of the Hanford formation H2 unit or the top of the fine-grained upper PlioPliestocene unit.

The ratio of PCi/g technetium-99 to $\mu \mathrm{g} / \mathrm{g}$ nitrate in the vadose zone sediment is fairly constant at about $100 \mathrm{PCi} / \mu \mathrm{g}$, which is similar to the ratio in the groundwater at borehole 299-W23-19 (see Johnson and Chou 1998). This ratio is also similar to that predicted for the tank supernates at the time of the SX115 leak. Despite the similarities in the technetium to nitrate ratio, there does not appear to be a continuous plume of these two constituents throughout the sediment profile to groundwater at this borehole. (There is however, some technetium-99 in all analyzed samples from the borehole.) Therefore, the pathway through the lower Plio-Pleistocene unit and Ringold Formation must be near to, but up hydraulic gradient from, well 299-W23-19.

Finally, there is an indication of very low concentrations ( 0.1 to $0.2 \mathrm{pCi} / \mathrm{g}$ ) of cesium-137 between 20.5 and 22.6 meters (67.45 and 74.25 feet bgs) in the vadose zone sediment at 299-W23-19. No significant cesium-137 plume was located in the vadose zone, though borehole 299-W23-19 is only 3 meters (10 feet) away from the south-south-west edge of tank SX-115 and about 17,000 curies of cesium137 is predicted to still exist from the 1965 leak. The position of the borehole is considered to be down structural gradient from the tank and, thus, if the leaked fluid followed the structural trend, any mobile cesium-137 should have been intercepted by borehole 299-W23-19. The data obtained from samples of borehole 299-W23-19, suggest that cesium-137 is fairly immobile at this borehole.

There is no indication that the vadose zone sediment in this borehole contains strontium-90, but there are small amounts of tritium between 21.3 (70 feet) and at least 38.1 meters (125 feet bgs). The tritium profile was not investigated in detail; therefore, it cannot be estimated how deep in the profile elevated tritium penetrated. The highest tritium activity is located at the contact between the Hanford formation $\mathrm{H} 2$ unit and the finer-grained, upper Plio-Pleistocene mud unit.

In summary, based on the water extract data, leaked tank fluids appear to have impacted the vadose zone at borehole 299-W23-19 from about 22.2 meters (73 feet bgs) to near the base of the upper PlioPleistocene 47.5 meters (156 feet bgs). Only the technetium-99 profile suggests that leaked fluids have penetrated the lower Plio-Pleistocene unit. The bulk of the leaked fluid currently resides in the upper Plio-Pleistocene unit between 38.1 and 47.5 meters 125 and 156 feet bgs). This is slightly deeper than the 
contamination investigated at tank SX-108 (Serne et al. 2002c) and at tank SX-109 (Serne et al. 2002b) both of which have the bulk of contamination in the Hanford formation H2 unit.

\subsection{Estimate of Sediment Recharge Rates (Matric Potential)}

To assess the water status in the sediment, we measured the soil suction on about 10 sleeves from the sediment profile at borehole 299-W23-19, although only one sample was from below the Plio-Pleistocene unit where we suggest the plume has reached. The measured soil suction values for the 10 sleeves from borehole 299-W23-19 are low (i.e., sediment is relatively wet) throughout the profile and drainage is predicted to be occurring.

There is no substitute for actual hydraulic conductivity measurements as a function of moisture content or direct measurement of recharge in the field, however using our simple models and years of data for the Hanford Site water balance, an estimated recharge rate of between 50 to 100 millimeters per year would be in line with the observed soil suction measurements on the cores from borehole 299-W23-19.

Currently, the source of soil moisture in borehole 299-W23-19, in excess of that occurring in uncontaminated boreholes outside the tank farm, cannot be determined unequivocally from the available data. Potential sources include natural precipitation that is concentrated due to man-induced changes in the surface soil inside the tank farm, leaking water lines, tank leaks or a combination of these.

\subsection{Detailed Characterization to Clarify Controlling Geochemical Processes}

Detailed characterization of selected sediment samples from borehole 299-W23-19 adds some insight to the processes controlling the vertical distribution of contaminants and their future migration potential. The first key finding is that the 1:1 sediment-to-water extracts give a good estimate of the sediment porewater chemistry. This is illustrated by the close agreement between concentrations derived from the dilution-corrected, water extracts and concentrations measured from actual porewater obtained from highspeed centrifugation. Constituents showing the best agreement are electrical conductivity, nitrate, sodium, and technetium concentrations. Tritium also would probably show good correlation but tritium was not studied in detail at borehole 299-W23-19.

The finding that water extract and ultracentrifuged samples are comparable is important because it is much easier to obtain water extract samples and it is now known that they can represent actual porewater.

The actual porewaters in the sediment from borehole 299-W23-19 were dominated by sodium and nitrate. The most concentrated porewater was essentially $0.3 \mathrm{M}$ sodium nitrate with several tenths molar concentrations of calcium and chromate.

The nitrite distribution suggests that, once leaked into the sediment, oxidation converts the nitrite to nitrate because the ratio of nitrite to nitrate is much lower in the sediment than in the tanks.

A key finding from this study is the lack of an observed, continuous vertical distribution of elevated nitrate or any other tank constituent from the tank bottom, through the entire vadose zone, to the water table at this borehole. There are small amounts of technetium-99 extending through the Ringold Formation to the water table and the deepest sample contains significant technetium-99, one indication of 
elevated technetium-99-rich porewater (176.2 pCi/mL dilution corrected) at 62.5 meters (205 feet bgs). The deepest sample may represent a zone influenced by technetium-99 in groundwater associated with a higher table in the past. Alternatively, the extract represents an analytical flyer. This sample was rather dry and the dilution factor to create the water extract was large. Therefore a small error in measurement causes a large error in the calculated porewater concentration.

The distribution of water extractable cations suggest that an ion-exchange process dominates the porewater/sediment interactions in the vadose zone where impacted by tank fluids. The leading edge of the tank leak plume is enriched in alkaline earth cations that were displaced from the native sediment exchange sites by sodium from the sodium-rich tank fluids.

Chromium does not migrate through the vadose zone as fast as technetium-99 and the leading edge of chromium contamination is near 38.4 meters (126 feet bgs) whereas that of the technetium-99 contamination is near 47.5 meters (156 feet bgs). The bulk of the water leachable chromium has been confirmed as chromium (VI) (chromate) by its distinct yellow color and by ion chromatography. There are at least two plausible mechanisms for the slight retardation of chromium (and selenium) as compared to technetium and molybdenum. The most probable is reduction of the highly mobile chromate (and selenate) ion. The second potential mechanism for immobilizing chromate and selenate is precipitation as an alkaline earth phase such as calcium chromate or calcium/barium mixed chromate/selenate/sulfate.

In summary, common ion exchange and heterogeneous (solid phase-liquid solute) redox reactions have been identified as two potential mechanisms influencing the distribution of contaminants in the vadose zone sediment at both boreholes 299-W23-19 and SX-41-09-39.

\subsection{Estimates of Sorption-Desorption Values}

A semiquantitative estimate of desorption $K_{d}$ values was calculated by combining the water extract and acid extract data. For a contaminant with very little water-soluble mass, such as uranium, the $\mathrm{K}_{\mathrm{d}} \mathrm{can}$ be approximated as the amount of mass on the solid (per gram of dry sediment) divided by the amount of mass in the porewater (per milliliter of porewater). For a contaminant that is very soluble in the water extract (equivalent to saying that the contaminant resides mainly in the porewater within the sediment), the amount of water-extractable contaminant must be subtracted from the total amount present in the sediment sample in order to obtain a value for the amount that would remain on the solid at equilibrium with the pore fluid. Using these measured distributions, the in situ desorption $\mathrm{K}_{\mathrm{d}}$ for uranium varies from 8 to $230 \mathrm{~mL} / \mathrm{g}$ in the sediment. For technetium, chromium, arsenic, selenium, and molybdenum, in the zone of elevated concentrations, the $K_{d}$ values vary between 0 and 0.09, 0.4 and 8, 2 and 6, 0.3 and 2, and 0.05 and $0.10 \mathrm{~mL} / \mathrm{g}$, respectively. Above and below the depths where contamination is obvious, in situ desorption $\mathrm{K}_{\mathrm{d}}$ values are larger because the pore fluids do not contain high sodium and nitrate concentrations competing for adsorption sites. Also, the bulk of the total trace metal, estimated from strong acid digests, is likely naturally present in the sediment from zones that are not obviously contaminated. That is, the naturally occurring trace metals are in forms that are resistant to water leaching whereas the tank derived contributions are water soluble.

Another technique to estimate contaminant mobility is comparison of the ratio of the concentration of a suspected contaminant to the concentration of a mobile contaminant such as technetium-99 or nitrate. As mentioned earlier, the ratios of technetium-99 to nitrate, chloride to nitrate and chloride to technetium- 
99 are fairly constant suggesting that these three constituents move together through the vadose zone. Ratios of concentrations of chromium, arsenic, selenium, and molybdenum to that of technetium or nitrate, show some degree of retardation since the ratios decrease with depth. The ratios decrease because the metals react more strongly with the vadose zone sediment than do technetium or nitrate and are removed from the percolating fluids. In general, the bulk of the reactive constituents chromium, molybdenum, selimium, arsenic, and sodium reacted with the vadose zone sediment in the bottom part of the laminated Hanford formation $\mathrm{H} 2$ unit sand or the top of the upper Plio-Pliestocene unit. There appears to be some discrepancy in the two techniques to estimate mobility for molybdenum. The in situ $\mathrm{K}_{\mathrm{d}}$ approach suggests that molybdenum is about as non-sorbing as technetium, but the ratio approach suggests that molybdenum is more reactive than technetium. However, differences in the assumptions for the two approaches may make the comparison inappropriate.

The technetium estimated $\mathrm{K}_{\mathrm{d}}$ from borehole 299-W23-19 measurements is consistent with technetium $\mathrm{K}_{\mathrm{d}} \mathrm{s}$ published elsewhere which find essentially no technetium adsorption onto Hanford Site sediment from less saline waters (Kaplan et al. 1995; Kaplan and Serne 2000).

Based on comparisons of the depths of penetration of the various contaminants and comparisons of the percentages of constituents that are water leachable, chromium appears to have migrated farther than cesium-137 but not as far as technetium-99 and nitrate. In other waste disposal situations at the Hanford Site, oxidized chromium, in reactor cooling water (low ionic strength and neutral $\mathrm{pH}$ ), appears to migrate similarly to technetium-99 and nitrate. All of these observations suggest that nitrate, technetium-99, and perhaps molybdenum migrate with no measurable retardation, whereas sodium, chromium, and selenium migrate with a small amount of retardation. These observations are remarkably similar to the conclusions found during the SX-41-09-39 borehole investigations.

Because very little cesium-137 was found in borehole 299-W23-19, the cesium-137 adsorption at this borehole appears to be quite large especially given the fact that the borehole is only 3 meters (10 feet) from the side of tank SX-115 and that the tank is estimated to have leaked 40,000 curies of cesium-137 into the ground. Only a trace (a few tenths of a pCi/g) of cesium-137 was found over a distance of about 2.1 meters ( 7 vertical feet) (20.5 to 22.6 meters [67.5 to 74.2 feet bgs]).

\subsection{Other Characterization Observations}

Comparing results from the $8 \mathrm{M}$ nitric acid digestions and the x-ray fluorescence analyses shows that the acid digestion removes less than about 10 to $25 \mathrm{wt} \%$ of the major elements and several minor elements (aluminum, calcium, magnesium, potassium, and strontium) and up to 40 to $70 \%$ of iron, manganese, and many trace metals. Although the database of elemental compositions of Hanford Site sediment is not large, comparison of sediment in borehole 299-W23-19 with those from the two uncontaminated boreholes (Serne et al. 2002a), the contaminated sediment at borehole 299-W23-19 in the H1a unit are slightly lower in aluminum oxide, sodium oxide, and magnesium oxide. In the Hanford formation $\mathrm{H} 1$ unit, the contaminated sediment is slightly lower in aluminum oxide, magnesium oxide, calcium oxide, titanium oxide, and ferric oxide and higher in potassium oxide and silica. In the Hanford formation $\mathrm{H} 2$ unit, contaminated sediment is depleted in aluminum oxide and magnesium oxide. Contaminated sediment in the upper Plio-Pleistocene unit does not differ substantially in composition from similar sediment in uncontaminated boreholes. 
$\mathrm{X}$-ray fluorescence is a more productive method to determine the total elemental concentrations of major and minor elements in vadose zone sediment than is the acid extract method. However, for situations in which the mass of a reactive constituent added to the vadose zone sediment by tank leak fluids is not dramatically large, the acid extracts may be useful. For example, at borehole 299-W23-19, the total measured sodium oxide content of the vadose zone sediment by $\mathrm{x}$-ray fluorescence does not show that the sediment is contaminated. However, both acid and water extracts of the same sediment clearly show an anthropomorphic contribution to the sodium. The lesson learned is that for natural sediment containing large concentrations of an element, an enormous contribution from the tank leak is required for the contamination to be observed by x-ray fluorescence analysis of the bulk sediment. There is no question that $\mathrm{x}$-ray fluorescence analyses gives valuable information for quantitative mineralogic investigations that have a strong relevance to studies of sediment-contaminated fluid interactions, but it is not equivocally shown that x-ray fluorescence analyses identify all trace metals present in levels elevated above the natural amounts.

Key parameters that may have an influence on contaminant migration are calcium carbonate content, particle size distribution, and bulk and clay size mineralogy. These parameters were measured on samples form borehole 299-W23-19. Cation exchange capacity of the bulk sediment was not measured because no contaminant cation of interest was found in the vadose zone sediment and there was no evidence that the mineralogy of contaminated sediment was altered by interactions with tank fluids. For these reasons, the cation exchange capacity of sediment from borehole 299-W23-19 is probably similar to that of uncontaminated boreholes described in Serne et al. 2002a.

X-ray diffraction analyses of the bulk samples from eight depths in borehole 299-W23-19 indicate that the sediment is mostly quartz ( 40 to 55\%) and feldspar ( 25 to 65\%), with lesser amounts of mica, chlorite, and smectite. Plagioclase feldspar is 2 to 6 times more abundant than potassium feldspar, with the exception of sample from a depth of 26.7 to 27 meters (87.6 to 88.6 feet), which has more potassium feldspar than plagioclase feldspar. Minor amounts of amphibole and calcite were also detected in the sediment.

Overall, smectite, illite, and chlorite are the dominant minerals in the clay fractions and total between 45 and $95 \mathrm{wt} \%$. Smectite, ranged in concentration from as high as $35 \mathrm{wt} \%$ to as low as $20 \mathrm{wt} \%$; illite occurred between about 10 and $30 \mathrm{wt} \%$. Chlorite concentrations were as low as $10 \mathrm{wt} \%$ and as high as 30 wt $\%$. Minor amounts of kaolinite ( $<10 \mathrm{wt} \%$ ) were detected at all depths. Quartz and feldspar made up about 5 to $15 \mathrm{wt} \%$ of the clay fraction. Iron oxides were detected in only trace amounts by transmission electron microscopy analysis of the clay fraction, suggesting that most of the iron in the $<2$ micron fraction is incorporated into the clay minerals. Based on x-ray fluorescence data, quantitative estimates of illite in the $<2$ micron fraction (assuming all $\mathrm{K}$ resides in the illite structure) are in good agreement with the $\mathrm{x}$-ray diffraction semiquantitative results.

Mineral assemblages, concentrations, and structural formulas were similar to data reported earlier on uncontaminated sediment from the Waste Management Area S-SX (Serne et al. 2002a). No evidence was observed of caustic tank liquor reacting with the sediment and altering the mineral phases or the mineral properties. 


\subsection{References}

ASTM. 1986. Standard method for particle size analysis soils. Annual Book of ASTM Standards. D42263 (R1972). American Society for Testing and Materials, Philadelphia, Pennsylvania.

ASTM. 1993. Standard practice for description and identification of soils (visual-manual procedure). D2488-93. American Society for Testing and Materials, West Conshohocken, Pennsylvania.

ASTM. 1997. Standard Test Method for Alkalinity in Brackish Water, Seawater, and Brines. Vol. 11.02. D3875-97. American Society for Testing and Materials, West Conshohocken, Pennsylvania.

ASTM. 1982. A Standard Test Method for Total and Organic Carbon in Water Oxidation by Coulometric Detection. D4129-82. American Society for Testing and Materials, West Conshohocken, Pennsylvania.

ASTM. 1988. Standard methods for total and organic carbon in water by high temperature oxidation and by coulometric detection. D4129-88. American Society for Testing and Materials, West Conshohocken, Pennsylvania.

American Society of Agronomy. 1986b. "Pynchnometer Method." Chapter 14-3 in Methods of soil analysis-Part 1, $2^{\text {nd }}$ edition of Physical and mineralogical methods, SSSA Book Series no. 5, ed. A Klute, pp. 378-379. Soil Science Society of America, Madison, Wisconsin.

American Society of Agronomy. 1986a. "Hydrometer Method." Chapter 15-5 in Methods of soil analysisPart 1, $2^{\text {nd }}$ edition of Physical and mineralogical methods, SSSA Book Series no. 5, ed. A Klute, pp. 404408. Soil Science Society of America, Madison, Wisconsin.

American Society of Agronomy. 1996a. "Elemental Analysis by XRF Spectroscopy." Chapter 7 in Methods of soil analysis- Part 3, Chemical methods, SSSA Book Series 5, ed. DL Sparks, pp. 161-223. Soil Science Society of America, Madison, Wisconsin.

Brindley, GW and G Brown, eds. 1980. Crystal structures of clay minerals and their x-ray identification. Monograph No. 5, Mineralogical Society, London.

Campbell, GS and GW Gee. 1986. "Water Potential: Miscellaneous Methods.” In Methods of Soil Analysis Part 1, A Klude (ed.), pp.g 619-632. American Society of Agronomy, Madison, Wisconsin.

Deka, RN, M Wairiu, PW Mtakwa, CE Mullins, EM Veenendaal, and J Townend. 1995. "Use and accuracy of the filter-paper technique for measurement of soil matric potential." European Journal of Soil Science 16:233-238.

DOE (See US Department of Energy)

Drever, JI. 1973. "The preparation of oriented clay mineral specimens for X-ray diffraction analysis by a filter-membrane peel technique." Amer. Minerl. 58:553-554. 
EPA. 1986. Test Methods for Evaluating Solid Waste: Physical/Chemcial Methods, SW-846, Third Edition. U.S. Environmental Protection Agency, Office of Solid Waste and Emergency Response, Washington, D.C., available online at http://www.epa.gov/epaoswer/hazwaste/test/sw846.htm.

EPA/Method 6020. 2000. "Inductively Coupled Plasma-Mass Spectrometry." Test methods for evaluating solid waste: physical/chemical methods, SW-846. U.S. Environmental Protection Agency, Office of Solid Waste and Emergency Response, Washington, D.C., available online at http://www.epa.gov/epaoswer/hazwaste/test/sw846.htm.

Gee, GW, MJ Fayer, ML Rockhold, and MD Campbell. 1992. "Variations in recharge at the Hanford Stie." Northwest Sci. 60(4):237-250.

Jackson, ML 1969. Soil chemical analysis - advanced course-2 $2^{\text {nd }}$ Edition. Department of Soil Science, University of Wisconsin, Madison, Wisconsin.

Johnson, V and CJ Chou. 1998. Results of phase I groundwater quality assessment for single-shell tank Waste Management Areas S-SX at the Hanford Site. PNNL-11810, Pacific Northwest National Laboratory, Richland, Washington.

Johnson, VG, TE Jones, SP Reidel, and MI Wood. 1999. Subsurface conditions description for the S-SX Waste Management Area. HNF-4936, Rev. 0, Lockheed Martin Hanford Corporation, Richland, Washington.

Kaplan, DI, RJ Serne, and MG Piepho. 1995. Geochemical factors affecting radionuclide transport through near and far field at a low-level waste disposal site. PNL-10379, Pacific Northwest Laboratory, Richland, Washington.

Kaplan, DI, and RJ Serne. 2000. Geochemical data package for the Hanford immobilized low-activity tank waste performance assessment (ILAW-PA). PNNL-13037 Rev.1, Pacific Northwest National Laboratory, Richland, Washington.

Lindsey, KA. 1996, The Miocene to Pliocene Ringold formation and associated deposits of the ancestral Columbia River system, south-central Washington and north-central Oregon, Open-File Report 96-8, Washington Division of Geology and Earth Resources, Olympia, Washington.

Lindsey, KA, SE Kos, and KD Reynolds. 2000. Vadose zone geology of boreholes 299-W22-50 and 299W23-19 S-SX Waste Management Area Hanford Site, south-central Washington. RPP-6149 Rev. 0, Daniel B. Stephens \& Associates, Richland, Washington.

Myers, DA, DL Parker, G Gee, VG Johnson, GV Last, RJ Serne, and DJ Moak. 1998. Findings of the extension of borehole 41-09-39, 241-SX tank farm. HNF-2855, Lockheed Martin Hanford Company, Richland, Washington.

Pacific Northwest Laboratory (PNL). 1990. Procedures for groundwater investigations. PNL MA-567, Pacific Northwest Laboratory, Richland, Washington. 
Pacific Northwest National Laboratory (PNNL). 1997. Gamma energy analysis operation and instrument verification using the Genie2000 ${ }^{t m}$ support software. PNNL-RRL-01, Pacific Northwest National Laboratory, Richland, Washington.

Pacific Northwest National Laboratory. 1998. Inductively coupled plasma mass spectrometric (ICP-MS) analysis. PNNL-AGG-415, Pacific Northwest National Laboratory, Richland, Washington.

Pacific Northwest National Laboratory. 2000a. Energy dispersive $x$-ray fluorescence spectroscopy using the BEP approach with the Kevex 770/8000 system. AGG-RAL-XRF-1 Rev. 0, Pacific Northwest National Laboratory, Richland, Washington.

Randall, RR, and RK Price. 1999. Analysis techniques and monitoring results 241-SX drywell surveillance logs. HNF-3136, Rev. 0, Waste Management Northwest and Three Rivers Scientific, Richland, Washington.

Reynolds DM, and RC Reynolds, Jr. 1989. X-ray diffraction and the identification and analysis of clay minerals. Oxford University Press, New York.

Rhoades, JD. 1996. "Salinity: Electrical conductivity and total dissolved solids." In Methods of soil analysis Part 3. JM Bigham (ed.), pp. 417-435. American Society of Agronomy, Madison, Wisconsin.

Rockhold, ML, CS Simmons, and MJ Fayer. 1997. "An analytical solution technique for onedimensional, steady vertical water flow in layered soils." Water Resour. Res. 33: 897-902.

Serne, RJ, JM Zachara, and DS Burke. 1998. Chemical information on tank supernatants, Cs adsorption from tank liquids onto Hanrord sediment, and field observations of Cs migration from past tank leaks. PNNL-11495, Pacific Northwest National Laboratory, Richland, Washington.

Serne RJ, BN Bjornstad, HT Schaef, BA Williams, DC Lanigan, DG Horton, RE Clayton, AV Mitroshkov, VL LeGore, MJ O'Hara, CF Brown, KE Parker, IV Kutnyakov, JN Serne, GV Last, SC Smith, CW Lindenmeier, JM Zachara, and DS Burke. 2002a. Characterization of vadose zone sediment, Part 1: Uncontaminated RCRA borehole core samples and composite samples. PNNL-13757-1, Pacific Northwest National Laboratory, Richland, Washington.

Serne RJ, GV Last, GW Gee, HT Schaef, DC Lanigan, CW Lindenmeier, RE Clayton, VL LeGore, RD Orr, MJ O'Hara, CF Brown, DS Burke, AT Owen, IV Kutnyakov, and TC Wilson. 2002b. Characterization of vadose zone sediment, Part 3: Borehole 41-09-39 in the S-SX Waste Management Area. PNNL-13757-3, Pacific Northwest National Laboratory, Richland, Washington.

Serne RJ, GV Last, HT Schaef, DC Lanigan, CW Lindenmeier, CC Ainsworth, RE Clayton, VL LeGore, MJ O'Hara, CF Brown, RD Orr, IV Kutnyakov, TC Wilson, KB Wagnon, BA Williams, and DS Burke. 2002c. Characterization of vadose zone sediment, Part 4: Slant borehole SX-108 in the S-SX Waste Management Area. PNNL-13757-4, Pacific Northwest National Laboratory, Richland, Washington. 
Serne RJ, HT Schaef, BN Bjornstad, DC Lanigan, GW Gee, CW Lindenmeier, RE Clayton, VL LeGore, MJ O'Hara, CF Brown, RD Orr, GV Last, IV Kutnyakov, DS Burke, TC Wilson, and BA Williams. 2002. Geologic and geochemical data collected from vadose zone sediments from Borehole 299 W23-19 [SX -115] in the S/SX Waste Management Area and preliminary interpretations. PNNL-13757-2, Pacific Northwest National Laboratory, Richland, Washington.

Serne RJ, GV Last, GW Gee, HT Schaef, DC Lanigan, CW Lindenmeier, RE Clayton, VL LeGore, RD Orr, MJ O'Hara, CF Brown, DS Burke, AT Owen, IV Kutnyakov, and TC Wilson. 2002. Geologic and geochemical data collected from vadose zone sediments from Borehole SX 41-09-39 in the S/SX Waste Management Area and preliminary interpretations. PNNL-13757-3, Pacific Northwest National Laboratory, Richland, Washington.

Serne RJ, GV Last, HT Schaef, DC Lanigan, CW Lindenmeier, CC Ainsworth, RE Clayton, VL LeGore, MJ O'Hara, CF Brown, RD Orr, IV Kutnyakov, TC Wilson, KB Wagnon, BA Williams, and DB Burke. 2002. Geologic and geochemical data and preliminary interpretations of vadose zone sediment from Slant Borehole SX-108 in the S-SX Waste Management Area. PNNL-13757-4, Pacific Northwest National Laboratory, Richland, Washington.

Siemens. 1994. "Spectra 3000 reference manual: Setting up quantitative method (named .QAN) for SRS 3000.” Siemens Analytical X-Ray Instruments, Book 2. publisher, city, state

Sobczyk, SM. 2000. Subsurface interpretation of the SX tank farm Hanford Site, Washington based on gamma-ray logging. Nez Perce Tribe Environmental Restoration Waste Management Program, Lapwai, Idaho.

SSSA. 1997. Glossary of soil science terms. Soil Science Society of America, Madison, Wisconsin.

US Department of Energy. 1999. Phase 1 RCRA facility investigation/corrective measures study work plan for the SST Waste Management Areas. DOE/RL-99-36, Rev. 0, Richland, Washington. 


\section{Distribution}

No. of

Copies

ONSITE

CH2M HILL Hanford Group, Inc.

Anthony J. Knepp (10 CD)

Fredrick M. Mann

U.S. Department of Energy

Robert M. Yasek

U.S. Department of Ecology

Joseph Caggiano
No. of

Copies

Pacific Northwest National Laboratory

R. Jeff Serne (50 CD) K6-81

Clark W. Lindenmeier P8-37

George V. Last K6-81

Bruce N. Bjornstad K6-81

Duane G. Horton K6-81

H. Todd Schaef K6-81

H6-60

B5-18

1 hard copy and $1 \mathrm{CD}$ except as noted.

Distr.1 